\title{
Supporting Information: A Mechanistic Study of Enhanced Protonation by Chromium(III) in Electrospray Ionization: A Superacid Bound to a Peptide
}

Rudradatt R. Persaud, Nnenna E. Dieke, Xinyao Jing, Skyler Lambert, Nicholas Parsa, Elizabeth Hartmann, John B. Vincent, Carolyn J. Cassady, and David A. Dixon

Department of Chemistry \& Biochemistry, The University of Alabama, Tuscaloosa, Alabama 35487-0336, USA

\section{Complete References}

41. Frisch, M.J., Trucks, G.W., Schlegel, H.B., Scuseria, G.E., Robb, M.A., Cheeseman, J.R., Scalmani, G., Barone, V., Mennucci, B., Petersson, G.A., Nakatsuji, H., Caricato, M., Li, X., Hratchian, H.P., Izmaylov, A.F., Bloino, J., Zheng, G., Sonnenberg, J.L., Hada, M., Ehara, M., Toyota, K., Fukuda, R., Hasegawa, J., Ishida, M., Nakajima, T., Honda, Y., Kitao, O., Nakai, H., Vreven, T., Montgomery, Jr., J.A., Peralta, J.E., Ogliaro, F., Bearpark, M., Heyd, J.J., Brothers, E., Kudin, K.N., Staroverov, V.N., Kobayashi, R., Normand, J., Raghavachari, K., Rendell, A., Burant, J.C., Iyengar, S.S., Tomasi, J., Cossi, M., Rega, N., Millam, N.J., Klene, M., Knox, J.E., Cross, J.B., Bakken, V., Adamo, C., Jaramillo, J., Gomperts, R., Stratmann, R.E., Yazyev, O., Austin, A.J., Cammi, R., Pomelli, C., Ochterski, J.W., Martin, R.L., Morokuma, K., Zakrzewski, V.G., Voth, G.A., Salvador, P., Dannenberg, J.J., Dapprich, S., Daniels, A.D., Farkas, Ö., Foresman, J.B., Ortiz, J.V., Cioslowski, J., Fox, D.J.: Gaussian 09 (Revision B.01). Gaussian, Inc., Wallingford CT (2009) 
Table S1. Calculated acidities of $\left[\mathrm{Met}(\mathrm{III})\left(\mathrm{H}_{2} \mathrm{O}\right)_{\mathrm{z}}\left(\mathrm{H}_{2} \mathrm{O}\right)_{2 \mathrm{z}}\right]^{3+}$ and $\left[\mathrm{Met}(\mathrm{III})\left(\mathrm{H}_{2} \mathrm{O}\right)_{\mathrm{z}}\right]^{3+}$ complexes in $\mathrm{kcal} / \mathrm{mol}$ and $\mathrm{p} K_{\mathrm{a}}$ 's at $298 \mathrm{~K}$ (full range values, repeat values omitted).

\begin{tabular}{|c|c|c|c|c|}
\hline Reaction & $\Delta \mathbf{H}_{\text {gas }}$ & $\Delta \mathbf{G}_{\text {gas }}$ & $\Delta \mathbf{G}_{\mathrm{aq}}$ & $\mathbf{p} \boldsymbol{K}_{\mathrm{a}}$ \\
\hline \multicolumn{5}{|l|}{${ }^{4}$ Chromium(III) } \\
\hline \multirow{5}{*}{$\begin{array}{l}{\left[{ }^{4} \mathrm{Cr}\left(\mathrm{H}_{2} \mathrm{O}\right)_{6}\left(\mathrm{H}_{2} \mathrm{O}\right)_{12}\right]^{3+} \rightarrow} \\
{\left[{ }^{4} \mathrm{Cr}\left(\mathrm{H}_{2} \mathrm{O}\right)_{5}(\mathrm{OH})\left(\mathrm{H}_{2} \mathrm{O}\right)_{12}\right]^{2+}+\mathrm{H}^{+}}\end{array}$} & 118.3 & 114.5 & 1.3 & 1.0 \\
\hline & 117.0 & 115.9 & 2.7 & 2.0 \\
\hline & 122.3 & 117.9 & 2.9 & 2.2 \\
\hline & 123.0 & 118.7 & 5.8 & 4.2 \\
\hline & 122.9 & 119.5 & 7.5 & 5.5 \\
\hline \multirow{2}{*}[{}^{4}\mathrm{Cr}(\mathrm{H}_{2}\mathrm{O})_{6}]{$^{3+} \rightarrow\left[{ }^{4} \mathrm{Cr}\left(\mathrm{H}_{2} \mathrm{O}\right)_{5}(\mathrm{OH})\right]^{2+}+\mathrm{H}^{+}$} & 29.7 & 20.1 & -14.8 & -10.9 \\
\hline & 29.6 & 19.3 & -14.5 & -10.6 \\
\hline \multicolumn{5}{|l|}{${ }^{1}$ Aluminum(III) } \\
\hline \multirow{4}{*}{$\begin{array}{l}{\left[{ }^{1} \mathrm{Al}\left(\mathrm{H}_{2} \mathrm{O}\right)_{6}(\mathrm{H} 2 \mathrm{O})_{12}\right]^{3+} \rightarrow} \\
{\left[{ }^{1} \mathrm{Al}\left(\mathrm{H}_{2} \mathrm{O}\right)_{5}(\mathrm{OH})\left(\mathrm{H}_{2} \mathrm{O}\right)_{12}\right]^{2+}+\mathrm{H}^{+}}\end{array}$} & 121.9 & 118.4 & 4.4 & 3.2 \\
\hline & 121.5 & 118.1 & 6.5 & 4.8 \\
\hline & 122.9 & 116.1 & 5.2 & 3.8 \\
\hline & 121.9 & 118.5 & 9.0 & 6.6 \\
\hline \multirow{2}{*}[{}^{1}\mathrm{Al}(\mathrm{H}_{2}\mathrm{O})_{6}]{$^{3+} \rightarrow\left[{ }^{1} \mathrm{Al}\left(\mathrm{H}_{2} \mathrm{O}\right)_{5}(\mathrm{OH})\right]^{2+}+\mathrm{H}^{+}$} & 33.2 & 23.8 & -9.2 & -6.8 \\
\hline & 33.3 & 23.1 & -8.9 & -6.6 \\
\hline \multicolumn{5}{|l|}{${ }^{6} \operatorname{Iron}(\mathrm{III})$} \\
\hline \multirow{3}{*}{$\begin{array}{l}{\left[{ }^{6} \mathrm{Fe}\left(\mathrm{H}_{2} \mathrm{O}\right)_{6}(\mathrm{H} 2 \mathrm{O})_{12}\right]^{3+} \rightarrow} \\
{\left[{ }^{6} \mathrm{Fe}\left(\mathrm{H}_{2} \mathrm{O}\right)_{5}(\mathrm{OH})\left(\mathrm{H}_{2} \mathrm{O}\right)_{12}\right]^{2+}+\mathrm{H}^{+}}\end{array}$} & 120.1 & 114.7 & -0.1 & -0.1 \\
\hline & 123.0 & 113.8 & 1.1 & 0.8 \\
\hline & 122.2 & 116.9 & 6.6 & 4.9 \\
\hline \multirow{2}{*}[{}^{6}\mathrm{Fe}(\mathrm{H}_{2}\mathrm{O})_{6}]{$^{3+} \rightarrow\left[{ }^{6} \mathrm{Fe}\left(\mathrm{H}_{2} \mathrm{O}\right)_{5}(\mathrm{OH})\right]^{2+}+\mathrm{H}^{+}$} & 21.6 & 11.5 & -18.5 & -13.5 \\
\hline & 21.6 & 11.5 & -18.3 & -13.4 \\
\hline${ }^{1}$ Scandium(III) & & & & \\
\hline
\end{tabular}




\begin{tabular}{|c|c|c|c|c|}
\hline \multirow{3}{*}{$\begin{array}{l}{\left[{ }^{1} \mathrm{Sc}\left(\mathrm{H}_{2} \mathrm{O}\right)_{6}\left(\mathrm{H}_{2} \mathrm{O}\right)_{12}\right]^{3+} \rightarrow} \\
{\left[{ }^{1} \mathrm{Sc}\left(\mathrm{H}_{2} \mathrm{O}\right)_{5}(\mathrm{OH})\left(\mathrm{H}_{2} \mathrm{O}\right)_{12}\right]^{2+}+\mathrm{H}^{+}}\end{array}$} & 123.8 & 116.8 & 1.4 & 1.0 \\
\hline & 124.4 & 118.4 & 3.6 & 2.7 \\
\hline & 127.9 & 118.6 & 6.1 & 4.5 \\
\hline \multirow{6}{*}[{}^{1}\mathrm{Sc}(\mathrm{H}_{2}\mathrm{O})_{6}]{$^{3+} \rightarrow\left[{ }^{1} \mathrm{Sc}\left(\mathrm{H}_{2} \mathrm{O}\right)_{5}(\mathrm{OH})\right]^{2+}+\mathrm{H}^{+}$} & 31.2 & 22.1 & -13.4 & $\begin{array}{c}-10.7 \\
\text { (to }-9.8 \text { ) }\end{array}$ \\
\hline & 31.2 & 21.3 & -14.2 & -10.4 \\
\hline & 31.2 & 21.6 & -13.9 & -10.2 \\
\hline & 31.2 & 21.9 & -13.6 & -10.0 \\
\hline & 31.1 & 21.9 & -13.5 & -9.9 \\
\hline & 31.2 & 22.1 & -13.4 & -9.8 \\
\hline \multicolumn{5}{|l|}{${ }^{1}$ Yttrium(III) } \\
\hline \multirow{6}{*}[{}^{1}\mathrm{Y}(\mathrm{H}_{2}\mathrm{O})_{24}]{$^{3+} \rightarrow\left[{ }^{1} \mathrm{Y}\left(\mathrm{H}_{2} \mathrm{O}\right)_{23}(\mathrm{OH})\right]^{2+}+\mathrm{H}^{+}$} & 138.5 & 140.7 & 11.1 & $\begin{array}{c}8.2 \text { (to } 10.5) \\
{[7.7]^{\mathrm{b}}}\end{array}$ \\
\hline & 150.8 & 146.6 & 12.8 & 9.4 \\
\hline & 137.6 & 142.0 & 13.0 & 9.5 \\
\hline & 135.6 & 142.1 & 13.7 & 10.0 \\
\hline & 137.9 & 143.4 & 13.7 & 10.1 \\
\hline & 143.5 & 143.1 & 14.3 & 10.5 \\
\hline \multirow{2}{*}[{}^{1}\mathrm{Y}(\mathrm{H}_{2}\mathrm{O})_{8}]{$^{3+} \rightarrow\left[{ }^{1} \mathrm{Y}\left(\mathrm{H}_{2} \mathrm{O}\right)_{7}(\mathrm{OH})\right]^{2+}+\mathrm{H}^{+}$} & 70.4 & 59.4 & 2.8 & 2.1 \\
\hline & 71.4 & 60.5 & 3.4 & 2.5 \\
\hline \multicolumn{5}{|l|}{${ }^{1}$ Lanthanum(III) } \\
\hline \multirow{5}{*}[{}^{1}\mathrm{La}(\mathrm{H}_{2}\mathrm{O})_{27}]{$^{3+} \rightarrow\left[{ }^{1} \mathrm{La}\left(\mathrm{H}_{2} \mathrm{O}\right)_{26}(\mathrm{OH})\right]^{2+}+\mathrm{H}^{+}$} & 149.2 & 143.6 & 8.6 & 6.3 \\
\hline & 152.1 & 146.6 & 9.0 & 6.6 \\
\hline & 153.1 & 147.6 & 9.1 & 6.7 \\
\hline & 162.0 & 150.1 & 11.4 & 8.4 \\
\hline & 156.9 & 149.4 & 12.4 & 9.1 \\
\hline
\end{tabular}




\begin{tabular}{|l|c|c|c|c|}
\hline & 156.8 & 150.0 & 12.8 & 9.4 \\
\cline { 2 - 5 } & 162.6 & 152.8 & 13.8 & 10.1 \\
\hline \multirow{2}{*}[{}^{[}\mathrm{La}(\mathrm{H}_{2}\mathrm{O})_{9}]{$^{3+} \rightarrow\left[{ }^{1} \mathrm{La}\left(\mathrm{H}_{2} \mathrm{O}\right)_{8}(\mathrm{OH})\right]^{2+}+\mathrm{H}^{+}$} & 83.7 & 71.5 & 4.6 & 3.4 \\
\cline { 2 - 5 } & 83.7 & 72.5 & 5.6 & 4.1 \\
\cline { 2 - 5 } & 84.0 & 73.2 & 6.1 & 4.5 \\
\hline
\end{tabular}


Table S2. Gas-phase reaction energies of desolvating the Fe(III)-glycine (neutral) and glycine (anion) complexes in $\mathrm{kcal} / \mathrm{mol}$.

\begin{tabular}{|l|c|c|c|c|c|c|}
\hline Complex $^{\mathbf{a}}$ & $\boldsymbol{\Delta H}_{\mathbf{2 9 8}} \mathbf{( 5 )}$ & $\boldsymbol{\Delta G}_{\mathbf{2 9 8}} \mathbf{( 5 )}$ & $<\mathbf{S}^{\mathbf{2}>\mathbf{b}}$ & $\mathbf{\Delta H}_{\mathbf{2 9 8}}(\mathbf{6})$ & $\boldsymbol{\Delta G}_{\mathbf{2 9 8}}(\mathbf{6})$ & $<\mathbf{S}^{\mathbf{2}>\mathbf{b}}$ \\
\hline Glycine, $\mathrm{x}=4$ & -336.8 & -347.9 & 8.76 & -46.6 & -56.2 & 8.76 \\
\hline Glycine, $\mathrm{x}=3$ & -298.0 & -320.7 & 8.76 & -9.5 & -30.8 & 8.76 \\
\hline Glycine, $\mathrm{x}=2$ & -268.0 & -299.7 & 8.76 & 42.9 & 11.9 & 8.76 \\
\hline Glycine, $\mathrm{x}=1$ & -225.6 & -265.5 & 8.76 & 115.9 & 75.6 & 8.76 \\
\hline Glycine, $\mathrm{x}=0$ & -167.8 & -217.2 & 8.76 & 208.7 & 158.7 & 8.76 \\
\hline
\end{tabular}

${ }^{a}$ Glycine complexed with ${ }^{6} \mathrm{Fe}(\mathrm{III})\left(\mathrm{H}_{2} \mathrm{O}\right)_{\mathrm{x}} \cdot{ }^{\text {b }}$ Ideal $<\mathrm{S}^{2}>$ value is 8.75 .

Table S3. Gas-phase reaction energies of desolvating the Al(III)-glycine (neutral) and glycine (anion) complexes in $\mathrm{kcal} / \mathrm{mol}$.

\begin{tabular}{|l|c|c|c|c|c|c|}
\hline Complex $^{\mathbf{a}}$ & $\mathbf{\Delta H}_{\mathbf{2 9 8}} \mathbf{( 5 )}$ & $\boldsymbol{\Delta G}_{\mathbf{2 9 8}} \mathbf{( 5 )}$ & $<\mathbf{S}^{\mathbf{2}>\mathbf{b}}$ & $\mathbf{\Delta H}_{\mathbf{2 9 8}}(\mathbf{6})$ & $\mathbf{\Delta G}_{\mathbf{2 9 8}}(\mathbf{6})$ & $<\mathbf{S}^{\mathbf{2}>\mathbf{b}}$ \\
\hline Glycine, $\mathrm{x}=4$ & -325.0 & -335.5 & 0 & -42.9 & -52.4 & 0 \\
\hline Glycine, $\mathrm{x}=3$ & -273.7 & -294.8 & 0 & -4.2 & -24.9 & 0 \\
\hline Glycine, $\mathrm{x}=2$ & -223.3 & -255.1 & 0 & 56.9 & 25.9 & 0 \\
\hline Glycine, $\mathrm{x}=1$ & -152.0 & -195.0 & 0 & 144.0 & 102.4 & 0 \\
\hline Glycine, $\mathrm{x}=0$ & -83.5 & -135.6 & 0 & 265.4 & 214.2 & 0 \\
\hline
\end{tabular}

${ }^{\mathrm{a}}$ Glycine complexed with ${ }^{1} \mathrm{Al}(\mathrm{III})\left(\mathrm{H}_{2} \mathrm{O}\right)_{\mathrm{x}} \cdot{ }^{\mathrm{b}}$ Ideal $<\mathrm{S}^{2}>$ value is 0 .

Table S4. Gas-phase reaction energies of desolvating the Sc(III)-glycine (neutral) and glycine (anion) complexes in $\mathrm{kcal} / \mathrm{mol}$.

\begin{tabular}{|l|c|c|c|c|c|c|}
\hline Complex $^{\mathbf{a}}$ & $\boldsymbol{\Delta} \mathbf{H}_{\mathbf{2 9 8}}(\mathbf{5})$ & $\boldsymbol{\Delta} \mathbf{G}_{\mathbf{2 9 8}} \mathbf{( 5 )}$ & $<\mathbf{S}^{\mathbf{2}} \mathbf{>}$ & $\boldsymbol{\Delta} \mathbf{H}_{\mathbf{2 9 8}}(\mathbf{6})$ & $\Delta \mathbf{G}_{\mathbf{2 9 8}}(\mathbf{6})$ & $<\mathbf{S}^{\mathbf{2}}>^{\mathbf{b}}$ \\
\hline Glycine, $\mathrm{x}=4$ & -324.0 & -333.9 & 0 & -39.5 & -48.8 & 0 \\
\hline Glycine, $\mathrm{x}=3$ & -262.7 & -284.0 & 0 & 3.1 & -17.6 & 0 \\
\hline Glycine, $\mathrm{x}=2$ & -219.1 & -249.9 & 0 & 58.0 & 27.5 & 0 \\
\hline Glycine, $\mathrm{x}=1$ & -164.4 & -203.5 & 0 & 126.5 & 87.9 & 0 \\
\hline Glycine, $\mathrm{x}=0$ & -103.5 & -151.9 & 0 & 205.4 & 157.6 & 0 \\
\hline
\end{tabular}

${ }^{a}$ Glycine complexed with ${ }^{1} \mathrm{Sc}(\mathrm{III})\left(\mathrm{H}_{2} \mathrm{O}\right)_{\mathrm{x}} \cdot{ }^{\mathrm{b}}$ Ideal $<\mathrm{S}^{2}>$ value is 0 . 
Table S5. Corrected values for the loss of a proton from the $\mathrm{Al}(\mathrm{III})\left(\mathrm{H}_{2} \mathrm{O}\right)_{6}$ complex: The Gas Phase Acidity and pK of Metal/ $\mathrm{H}_{2} \mathrm{O}$ Complexes at the MP2/cc-pVDZ/aug-cc-pVDZ level at $298 \mathrm{~K}$.

\begin{tabular}{|c|c|c|c|c|c|c|c|c|}
\hline $\begin{array}{l}\text { Metal ion } / \mathrm{H}_{2} \mathrm{O} \\
\text { complex }\end{array}$ & $\begin{array}{c}\Delta \mathrm{G}_{298} \\
\text { gas }\end{array}$ & COSMO & $\begin{array}{l}\text { CPCM- } \\
\text { COSMO- } \\
\text { RS Radii }\end{array}$ & $\begin{array}{l}\text { IEF- } \\
\text { PCM } \\
\text { UA0 }\end{array}$ & $\begin{array}{c}\text { IEF-PCM } \\
\text { UFF }\end{array}$ & IPCM & $\begin{array}{l}\text { IEF-PCM- } \\
\text { Pauling }\end{array}$ & $\begin{array}{c}\text { CSC-PCM } \\
\text { Pauling }\end{array}$ \\
\hline$\left[\mathrm{Al}\left(\mathrm{H}_{2} \mathrm{O}\right)_{6}\right]^{3+}$ & 24.7 & -9.5 & -4.8 & -7.1 & -15.9 & -1.6 & -6.5 & -3.0 \\
\hline$\left[\mathrm{Al}\left(\mathrm{H}_{2} \mathrm{O}\right)_{6}\left(\mathrm{H}_{2} \mathrm{O}\right)_{12}\right]^{3+}$ & 120.3 & 3.7 & 6.6 & 4.9 & 1.1 & 8.5 & 5.1 & 6.2 \\
\hline
\end{tabular}


Table S6. Cartesian coordinates in angstroms and electronic energies (hartree) of structures in Figure 2 at the B3LYP/DZVP2 level for C, H, O, N, and cc-pVDZ-PP for Cr(III).

$\left[(\mathrm{Glycine}){ }^{4} \mathrm{Cr}\left(\mathrm{H}_{2} \mathrm{O}\right)_{3}\left(\mathrm{OH}^{-}\right)\right]^{+2}$ (a)

$\mathrm{E}=-675.8636629$

$\begin{array}{lccc}\mathrm{C} & 1.474613 & -0.058266 & 0.417153 \\ \mathrm{C} & 2.973108 & -0.110926 & 0.673295 \\ \mathrm{O} & 1.036836 & 0.001412 & -0.785395 \\ \mathrm{~N} & 3.727332 & 0.106446 & -0.620264 \\ \mathrm{H} & 3.271786 & 0.650329 & 1.396217 \\ \mathrm{H} & 3.242119 & -1.092709 & 1.070295 \\ \mathrm{O} & 0.651605 & -0.092723 & 1.378144 \\ \mathrm{H} & 3.691551 & 1.088158 & -0.919909 \\ \mathrm{H} & 4.716178 & -0.155936 & -0.524317 \\ \mathrm{H} & 3.308761 & -0.448849 & -1.378785 \\ \mathrm{O} & -2.630271 & 0.037127 & 1.042108 \\ \mathrm{H} & -3.457123 & 0.040131 & 0.525858 \\ \mathrm{H} & -2.830014 & -0.162298 & 1.971523 \\ \mathrm{O} & -0.907266 & -2.051083 & -0.161309 \\ \mathrm{H} & -1.520788 & -2.416191 & -0.824544 \\ \mathrm{H} & -0.864080 & -2.666790 & 0.589682 \\ \mathrm{O} & -0.811273 & 2.054056 & 0.017496 \\ \mathrm{H} & -1.386257 & 2.479390 & -0.645306 \\ \mathrm{H} & -0.894945 & 2.554190 & 0.847759 \\ \mathrm{CR} & -0.884774 & 0.001176 & -0.070659 \\ \mathrm{O} & -1.981522 & 0.084822 & -1.513141 \\ \mathrm{H} & -1.685143 & 0.103472 & -2.436718\end{array}$

$\left[(\text { Glycine })^{4}{ }^{4} \mathrm{Cr}\left(\mathrm{H}_{2} \mathrm{O}\right)_{4}\right]^{+2}$ (b)

$\mathrm{E}=-675.836075$

$\begin{array}{rrrr}\mathrm{C} & -1.523938 & -0.451568 & 0.016819 \\ \mathrm{C} & -3.014484 & -0.660457 & 0.007918 \\ \mathrm{O} & -0.808919 & -0.280097 & -1.060724 \\ \mathrm{~N} & -3.509237 & 0.724267 & -0.039137 \\ \mathrm{H} & -3.310794 & -1.194638 & 0.913436 \\ \mathrm{H} & -3.292934 & -1.245562 & -0.871875 \\ \mathrm{O} & -0.821986 & -0.348747 & 1.103476 \\ \mathrm{H} & -4.061134 & 0.907508 & -0.871168 \\ \mathrm{H} & -4.052098 & 0.965378 & 0.783989 \\ \mathrm{O} & 0.442170 & 2.051983 & -0.059230 \\ \mathrm{H} & -0.390697 & 2.383962 & -0.450025 \\ \mathrm{H} & 0.666329 & 2.642785 & 0.683129 \\ \mathrm{O} & 2.302647 & 0.385109 & -1.398382 \\ \mathrm{H} & 2.374611 & 1.220061 & -1.895777 \\ \mathrm{H} & 2.779491 & -0.299006 & -1.901822\end{array}$




$\begin{array}{lccc}\mathrm{O} & 1.245318 & -1.954542 & -0.185457 \\ \mathrm{H} & 0.764930 & -2.541213 & -0.799036 \\ \mathrm{H} & 1.574916 & -2.503615 & 0.549320 \\ \mathrm{O} & 2.106068 & 0.217563 & 1.591993 \\ \mathrm{H} & 3.063070 & 0.392538 & 1.541578 \\ \mathrm{H} & 1.860174 & 0.149064 & 2.533046 \\ \mathrm{CR} & 0.754039 & 0.006453 & -0.000944\end{array}$

$\left[(\text { Glycine }){ }^{4} \mathrm{Cr}\left(\mathrm{H}_{2} \mathrm{O}\right)_{4}\right]^{+3}$

$\mathrm{E}=-675.9684581$

$\begin{array}{cccc}\mathrm{C} & 2.977106 & -0.004197 & -0.635472 \\ \mathrm{H} & 3.213284 & 0.874215 & -1.245114 \\ \mathrm{H} & 3.213284 & -0.890336 & -1.233877 \\ \mathrm{C} & 1.490793 & -0.002290 & -0.322982 \\ \mathrm{O} & 0.636426 & -0.004606 & -1.275857 \\ \mathrm{O} & 0.995958 & 0.001445 & 0.864945 \\ \mathrm{CR} & -0.846606 & 0.000111 & 0.026448 \\ \mathrm{~N} & 3.860770 & 0.003573 & 0.590022 \\ \mathrm{H} & 3.724356 & -0.822666 & 1.188364 \\ \mathrm{H} & 4.851807 & 0.002364 & 0.300320 \\ \mathrm{O} & -0.930714 & 2.030532 & 0.100569 \\ \mathrm{O} & -0.933634 & -2.029699 & 0.111875 \\ \mathrm{O} & -2.195602 & 0.005559 & 1.570851 \\ \mathrm{H} & -2.537602 & 0.793582 & 2.036332 \\ \mathrm{O} & -2.330747 & -0.002900 & -1.376279 \\ \mathrm{H} & -3.295371 & -0.001632 & -1.216045 \\ \mathrm{H} & -2.187983 & -0.005763 & -2.344396 \\ \mathrm{H} & -0.471567 & 2.616727 & 0.732256 \\ \mathrm{H} & -1.332016 & 2.593477 & -0.590829 \\ \mathrm{H} & -2.538028 & -0.779248 & 2.041434 \\ \mathrm{H} & -1.336683 & -2.595723 & -0.575991 \\ \mathrm{H} & -0.474733 & -2.613223 & 0.746210 \\ \mathrm{H} & 3.723524 & 0.836841 & 1.178333\end{array}$

Geometries of gas-phase reaction energies of desolvating the $\mathrm{Cr}$ (III)-glycine (neutral) and glycine (anion) complexes (from Table 1).

Glycine, $\mathrm{x}=4$ (Reaction 6)

$\mathrm{E}=-675.8636629$

$\begin{array}{rrrr}\mathrm{C} & 1.474613 & -0.058266 & 0.417153 \\ \mathrm{C} & 2.973108 & -0.110926 & 0.673295 \\ \mathrm{O} & 1.036836 & 0.001412 & -0.785395 \\ \mathrm{~N} & 3.727332 & 0.106446 & -0.620264 \\ \mathrm{H} & 3.271786 & 0.650329 & 1.396217 \\ \mathrm{H} & 3.242119 & -1.092709 & 1.070295 \\ \mathrm{O} & 0.651605 & -0.092723 & 1.378144\end{array}$




$\begin{array}{lccc}\mathrm{H} & 3.691551 & 1.088158 & -0.919909 \\ \mathrm{H} & 4.716178 & -0.155936 & -0.524317 \\ \mathrm{H} & 3.308761 & -0.448849 & -1.378785 \\ \mathrm{O} & -2.630271 & 0.037127 & 1.042108 \\ \mathrm{H} & -3.457123 & 0.040131 & 0.525858 \\ \mathrm{H} & -2.830014 & -0.162298 & 1.971523 \\ \mathrm{O} & -0.907266 & -2.051083 & -0.161309 \\ \mathrm{H} & -1.520788 & -2.416191 & -0.824544 \\ \mathrm{H} & -0.864080 & -2.666790 & 0.589682 \\ \mathrm{O} & -0.811273 & 2.054056 & 0.017496 \\ \mathrm{H} & -1.386257 & 2.479390 & -0.645306 \\ \mathrm{H} & -0.894945 & 2.554190 & 0.847759 \\ \mathrm{CR} & -0.884774 & 0.001176 & -0.070659 \\ \mathrm{O} & -1.981522 & 0.084822 & -1.513141 \\ \mathrm{H} & -1.685143 & 0.103472 & -2.436718\end{array}$

Glycine, $\mathrm{x}=4$ (Reaction 7)

$\left[(\text { Glycine }){ }^{4} \mathrm{Cr}\left(\mathrm{H}_{2} \mathrm{O}\right)_{4}\right]^{+3}$

$\mathrm{E}=-675.9684581$

$\begin{array}{lccc}\mathrm{C} & 2.977106 & -0.004197 & -0.635472 \\ \mathrm{H} & 3.213284 & 0.874215 & -1.245114 \\ \mathrm{H} & 3.213284 & -0.890336 & -1.233877 \\ \mathrm{C} & 1.490793 & -0.002290 & -0.322982 \\ \mathrm{O} & 0.636426 & -0.004606 & -1.275857 \\ \mathrm{O} & 0.995958 & 0.001445 & 0.864945 \\ \mathrm{CR} & -0.846606 & 0.000111 & 0.026448 \\ \mathrm{~N} & 3.860770 & 0.003573 & 0.590022 \\ \mathrm{H} & 3.724356 & -0.822666 & 1.188364 \\ \mathrm{H} & 4.851807 & 0.002364 & 0.300320 \\ \mathrm{O} & -0.930714 & 2.030532 & 0.100569 \\ \mathrm{O} & -0.933634 & -2.029699 & 0.111875 \\ \mathrm{O} & -2.195602 & 0.005559 & 1.570851 \\ \mathrm{H} & -2.537602 & 0.793582 & 2.036332 \\ \mathrm{O} & -2.330747 & -0.002900 & -1.376279 \\ \mathrm{H} & -3.295371 & -0.001632 & -1.216045 \\ \mathrm{H} & -2.187983 & -0.005763 & -2.344396 \\ \mathrm{H} & -0.471567 & 2.616727 & 0.732256 \\ \mathrm{H} & -1.332016 & 2.593477 & -0.590829 \\ \mathrm{H} & -2.538028 & -0.779248 & 2.041434 \\ \mathrm{H} & -1.336683 & -2.595723 & -0.575991 \\ \mathrm{H} & -0.474733 & -2.613223 & 0.746210 \\ \mathrm{H} & 3.723524 & 0.836841 & 1.178333\end{array}$

Glycine, $\mathrm{x}=3$ (Reaction 6)

$\mathrm{E}=-599.3533479$

$\begin{array}{llll}\text { C } & 2.882325 & 0.647108 & -0.040792\end{array}$ 


$\begin{array}{llcc}\mathrm{H} & 3.115849 & 1.195845 & 0.874326 \\ \mathrm{H} & 3.194774 & 1.250096 & -0.897614 \\ \mathrm{C} & 1.392639 & 0.367709 & -0.121902 \\ \mathrm{O} & 0.548262 & 1.317793 & 0.013613 \\ \mathrm{O} & 0.951606 & -0.811803 & -0.319670 \\ \mathrm{CR} & -0.946338 & 0.002366 & -0.086184 \\ \mathrm{~N} & 3.656291 & -0.650871 & -0.050017 \\ \mathrm{H} & 3.385474 & -1.242310 & -0.846664 \\ \mathrm{H} & 4.667454 & -0.473454 & -0.108774 \\ \mathrm{O} & -1.149331 & -0.428419 & 1.630455 \\ \mathrm{O} & -2.252469 & -1.532516 & -0.526937 \\ \mathrm{H} & -2.501326 & -2.075980 & 0.244990 \\ \mathrm{O} & -2.414457 & 1.363550 & -0.414842 \\ \mathrm{H} & -2.298146 & 2.329255 & -0.351407 \\ \mathrm{H} & -3.367825 & 1.167752 & -0.362535 \\ \mathrm{H} & -1.285501 & 0.249964 & 2.314646 \\ \mathrm{H} & -2.394044 & -2.057984 & -1.333768 \\ \mathrm{H} & 3.482690 & -1.201617 & 0.800531\end{array}$

Glycine, $\mathrm{x}=3$ (Reaction 7)

$\mathrm{E}=-599.4296462$

$\begin{array}{lrrr}\mathrm{C} & 1.372615 & -0.316709 & -0.081315 \\ \mathrm{C} & 2.852887 & -0.640061 & -0.129290 \\ \mathrm{O} & 0.499668 & -1.262096 & -0.168219 \\ \mathrm{~N} & 3.755720 & 0.568766 & -0.079279 \\ \mathrm{H} & 3.059840 & -1.201887 & -1.047443 \\ \mathrm{H} & 3.092835 & -1.297724 & 0.714603 \\ \mathrm{O} & 0.871185 & 0.862507 & 0.038661 \\ \mathrm{H} & 3.638888 & 1.126417 & 0.778905 \\ \mathrm{H} & 4.741344 & 0.258650 & -0.099405 \\ \mathrm{H} & 3.629925 & 1.200243 & -0.883470 \\ \mathrm{O} & -1.963355 & 1.739349 & -0.522736 \\ \mathrm{H} & -1.589314 & 2.642877 & -0.447042 \\ \mathrm{H} & -2.872965 & 1.843613 & -0.873560 \\ \mathrm{O} & -1.314969 & -0.149833 & 1.823749 \\ \mathrm{H} & -1.536988 & 0.573711 & 2.447213 \\ \mathrm{H} & -1.393704 & -1.005620 & 2.296271 \\ \mathrm{O} & -2.370846 & -1.210300 & -0.782519 \\ \mathrm{H} & -2.160313 & -2.092385 & -1.158572 \\ \mathrm{H} & -3.347500 & -1.157453 & -0.718507 \\ \mathrm{CR} & -0.944941 & 0.042992 & -0.095912\end{array}$

Glycine, $\mathrm{x}=2$ (Reaction 6)

$\mathrm{E}=-522.836329$
C $\quad-1.153831-0.337307$
0.067152
$\begin{array}{llll}\text { C } & -2.600138 & -0.712535 & 0.323707\end{array}$ 


$\begin{array}{lccc}\mathrm{O} & -0.831248 & 0.745612 & -0.507612 \\ \mathrm{~N} & -3.521003 & 0.381882 & -0.162565 \\ \mathrm{H} & -2.760006 & -0.863908 & 1.394762 \\ \mathrm{H} & -2.845197 & -1.642982 & -0.195891 \\ \mathrm{O} & -0.200665 & -1.122518 & 0.443429 \\ \mathrm{H} & -3.342510 & 1.273703 & 0.318434 \\ \mathrm{H} & -4.503902 & 0.126272 & 0.003399 \\ \mathrm{H} & -3.407415 & 0.559155 & -1.169465 \\ \mathrm{O} & 1.698283 & 1.606912 & 0.541613 \\ \mathrm{H} & 2.375559 & 1.757092 & 1.229299 \\ \mathrm{CR} & 1.146057 & 0.146104 & -0.197202 \\ \mathrm{O} & 2.786873 & -1.029117 & -0.197829 \\ \mathrm{H} & 3.690971 & -0.760002 & -0.449891 \\ \mathrm{H} & 2.832010 & -1.937050 & 0.158201\end{array}$

Glycine, $\mathrm{x}=2$ (Reaction 7)

$\mathrm{E}=-522.8779237$

$\begin{array}{cccc}\mathrm{C} & -1.157504 & 0.229027 & 0.156058 \\ \mathrm{C} & -2.597291 & 0.561635 & 0.484684 \\ \mathrm{O} & -0.185141 & 0.897399 & 0.716111 \\ \mathrm{~N} & -3.620164 & -0.225976 & -0.296443 \\ \mathrm{H} & -2.755243 & 1.633236 & 0.306997 \\ \mathrm{H} & -2.751985 & 0.389516 & 1.558465 \\ \mathrm{O} & -0.739564 & -0.669058 & -0.656451 \\ \mathrm{H} & -3.571679 & -0.064257 & -1.314008 \\ \mathrm{H} & -4.567262 & 0.066912 & 0.000385 \\ \mathrm{H} & -3.566294 & -1.243134 & -0.136076 \\ \mathrm{O} & 1.746650 & -1.648366 & 0.565444 \\ \mathrm{H} & 2.476631 & -1.700109 & 1.225773 \\ \mathrm{H} & 1.339193 & -2.541660 & 0.490536 \\ \mathrm{O} & 2.705110 & 1.166097 & -0.188569 \\ \mathrm{H} & 2.828456 & 1.894986 & 0.462391 \\ \mathrm{H} & 3.509137 & 1.156440 & -0.758430 \\ \mathrm{CR} & 1.113022 & -0.030110 & -0.295736\end{array}$

Glycine, $\mathrm{x}=1$ (Reaction 6)

$E=-446.3049419$

$\begin{array}{lccc}\mathrm{C} & -2.579166 & 0.352025 & 0.000143 \\ \mathrm{H} & -2.955807 & 0.929335 & -0.868146 \\ \mathrm{H} & -2.955568 & 0.928791 & 0.868942 \\ \mathrm{C} & -1.059670 & 0.306696 & 0.000059 \\ \mathrm{O} & -0.381618 & 1.381915 & -0.000090 \\ \mathrm{O} & -0.431991 & -0.810330 & 0.000089 \\ \mathrm{CR} & 1.298319 & 0.281127 & -0.000078 \\ \mathrm{~N} & -3.161905 & -0.950713 & -0.000099 \\ \mathrm{H} & -2.565919 & -1.791215 & 0.000134\end{array}$




$\begin{array}{cccc}\mathrm{H} & -4.182705 & -1.074009 & -0.000445 \\ \mathrm{O} & 3.099714 & -0.697289 & 0.000070 \\ \mathrm{H} & 3.588562 & -1.016618 & -0.782964 \\ \mathrm{H} & 3.589304 & -1.015022 & 0.783290\end{array}$

Glycine, $\mathrm{x}=1$ (Reaction 7)

$\mathrm{E}=-446.303345$

$\begin{array}{lccc}\mathrm{C} & 0.835737 & -0.274149 & -0.005018 \\ \mathrm{C} & 2.287835 & -0.587144 & 0.279748 \\ \mathrm{O} & 0.312607 & 0.877680 & -0.294772 \\ \mathrm{~N} & 3.249419 & 0.570007 & 0.172191 \\ \mathrm{H} & 2.599066 & -1.386141 & -0.409659 \\ \mathrm{H} & 2.340436 & -1.012190 & 1.293739 \\ \mathrm{O} & -0.070845 & -1.208539 & 0.004520 \\ \mathrm{H} & 3.056367 & 1.337274 & 0.835566 \\ \mathrm{H} & 4.201711 & 0.228107 & 0.398046 \\ \mathrm{H} & 3.309569 & 0.974525 & -0.776055 \\ \mathrm{CR} & -1.390522 & 0.030547 & -0.543016 \\ \mathrm{O} & -2.494059 & 0.279794 & 1.018014 \\ \mathrm{H} & -2.728334 & -0.423658 & 1.674108 \\ \mathrm{H} & -2.875275 & 1.135174 & 1.340837\end{array}$

Glycine, $x=0$ (Reaction 6)

$\mathrm{E}=-369.7385643$
$\begin{array}{lll}\text { C } & 0.502124 & 0.275701\end{array}$
$\begin{array}{llll}\text { C } & 1.986008 & 0.601979 & 0.000137\end{array}$
$\begin{array}{llll}\mathrm{O} & 0.074383 & -0.932925 & 0.000142\end{array}$
$\mathrm{N} \quad 2.812081 \quad-0.564603 \quad-0.000168$
$\mathrm{H} \quad 2.244575 \quad 1.239990 \quad 0.869763$
$\mathrm{H} \quad 2.244831 \quad 1.240733 \quad-0.868813$
$\begin{array}{llll}\mathrm{O} & -0.371637 & 1.207502 & -0.000088\end{array}$
$\mathrm{H} \quad 2.406937 \quad-1.512575 \quad 0.000186$
H $\quad 3.838181 \quad-0.478108 \quad-0.000654$
$\begin{array}{llll}\text { CR } & -1.790411 & -0.166688 & -0.000032\end{array}$

Glycine, $\mathrm{x}=0$ (Reaction 7)

$\mathrm{E}=-369.6971882$
$\begin{array}{llll}\text { C } & -1.900471 & 0.640697 & 0.000094\end{array}$
$\mathrm{H} \quad-2.026875 \quad 1.298984 \quad-0.879324$
$\mathrm{H} \quad-2.026803 \quad 1.299305 \quad 0.879277$
C $\quad-0.471813 \quad 0.171131 \quad 0.000143$
$\begin{array}{lllll}\text { O } & 0.547248 & 1.098106 & -0.000259\end{array}$
$\begin{array}{llll}\text { O } & 0.039828 & -0.985059 & 0.000174\end{array}$
$\begin{array}{llll}\text { CR } & 1.839624 & -0.140540 & -0.000165\end{array}$

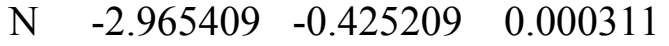
$\mathrm{H} \quad-2.954160 \quad-1.032351 \quad 0.838137$ 


$$
\begin{array}{cccc}
\mathrm{H} & -3.893982 & 0.040842 & 0.000229 \\
\mathrm{H} & -2.954187 & -1.032686 & -0.837272
\end{array}
$$

Geometries of calculated acidities of Cr(III) (from Table 2).

\begin{tabular}{cccc}
\multicolumn{5}{c}{$\left.{ }^{4} \mathrm{Cr}\left(\mathrm{H}_{2} \mathrm{O}\right)_{6}\left(\mathrm{H}_{2} \mathrm{O}\right)_{12}\right]^{3+}$} & \\
$\mathrm{E}=-1462.2798329$ & & \\
$\mathrm{CR}$ & 0.000000 & 0.000000 & -0.000020 \\
$\mathrm{O}$ & 0.000000 & 0.000000 & -1.999054 \\
$\mathrm{O}$ & 0.000000 & 2.031050 & -0.000042 \\
$\mathrm{O}$ & 0.000000 & 0.000000 & 1.999011 \\
$\mathrm{O}$ & 0.000000 & -2.031050 & -0.000042 \\
$\mathrm{O}$ & -1.974893 & 0.000003 & -0.000016 \\
$\mathrm{O}$ & 1.974893 & -0.000003 & -0.000016 \\
$\mathrm{H}$ & -2.555056 & -0.317270 & 0.763930 \\
$\mathrm{H}$ & -2.555062 & 0.317344 & -0.763926 \\
$\mathrm{H}$ & -0.588473 & 2.600288 & 0.550825 \\
$\mathrm{H}$ & 0.588575 & 2.600252 & -0.550835 \\
$\mathrm{H}$ & -0.654968 & -0.486216 & -2.574113 \\
$\mathrm{H}$ & 0.654968 & 0.486216 & -2.574113 \\
$\mathrm{H}$ & 0.588473 & -2.600288 & 0.550825 \\
$\mathrm{H}$ & -0.588575 & -2.600252 & -0.550835 \\
$\mathrm{H}$ & -0.654925 & 0.486273 & 2.574075 \\
$\mathrm{H}$ & 0.654925 & -0.486273 & 2.574075 \\
$\mathrm{H}$ & 2.555056 & 0.317270 & 0.763930 \\
$\mathrm{H}$ & 2.555062 & -0.317344 & -0.763926 \\
$\mathrm{O}$ & 1.590271 & 3.636855 & -1.693059 \\
$\mathrm{H}$ & 2.418027 & 4.011401 & -1.344450 \\
$\mathrm{O}$ & -1.589896 & 3.637010 & 1.693136 \\
$\mathrm{H}$ & -2.417590 & 4.011725 & 1.344562 \\
$\mathrm{O}$ & 1.589896 & -3.637010 & 1.693136 \\
$\mathrm{H}$ & 1.127117 & -4.389849 & 2.101136 \\
$\mathrm{O}$ & -1.590271 & -3.636855 & -1.693059 \\
$\mathrm{H}$ & -2.418027 & -4.011401 & -1.344450 \\
$\mathrm{H}$ & 1.127640 & 4.389798 & -2.101034 \\
$\mathrm{H}$ & -1.127117 & 4.389849 & 2.101136 \\
$\mathrm{H}$ & 2.417590 & -4.011725 & 1.344562 \\
$\mathrm{H}$ & -1.127640 & -4.389798 & -2.101034 \\
$\mathrm{O}$ & -3.398031 & -0.663033 & 2.064884 \\
$\mathrm{H}$ & -4.292040 & -1.024995 & 2.160093 \\
$\mathrm{O}$ & -3.398113 & 0.663196 & -2.064810 \\
$\mathrm{H}$ & -3.258301 & 0.011904 & -2.775988 \\
$\mathrm{H}$ & -3.258219 & -0.011682 & 2.776009 \\
$\mathrm{H}$ & -4.292111 & 1.025188 & -2.160002 \\
$\mathrm{O}$ & 3.398031 & 0.663033 & 2.064884 \\
$\mathrm{H}$ & 4.292040 & 1.024995 & 2.160093
\end{tabular}




$\begin{array}{rrrc}\mathrm{O} & 3.398113 & -0.663196 & -2.064810 \\ \mathrm{H} & 3.258301 & -0.011904 & -2.775988 \\ \mathrm{H} & 3.258219 & 0.011682 & 2.776009 \\ \mathrm{H} & 4.292111 & -1.025188 & -2.160002 \\ \mathrm{O} & 1.852064 & 1.343551 & -3.414385 \\ \mathrm{H} & 1.912288 & 2.244773 & -3.033779 \\ \mathrm{O} & -1.852064 & -1.343551 & -3.414385 \\ \mathrm{H} & -1.834208 & -1.447243 & -4.380398 \\ \mathrm{H} & 1.834208 & 1.447243 & -4.380398 \\ \mathrm{H} & -1.912288 & -2.244773 & -3.033779 \\ \mathrm{O} & -1.851980 & 1.343638 & 3.414316 \\ \mathrm{H} & -1.912136 & 2.244889 & 3.033770 \\ \mathrm{O} & 1.851980 & -1.343638 & 3.414316 \\ \mathrm{H} & 1.912136 & -2.244889 & 3.033770 \\ \mathrm{H} & -1.834174 & 1.447263 & 4.380337 \\ \mathrm{H} & 1.834174 & -1.447263 & 4.380337\end{array}$

$\left[{ }^{4} \mathrm{Cr}\left(\mathrm{H}_{2} \mathrm{O}\right)_{5}(\mathrm{OH})\left(\mathrm{H}_{2} \mathrm{O}\right)_{12}\right]^{2+}$ (a)

$\mathrm{E}=-1462.0756335$

$\begin{array}{cccc}\mathrm{O} & 1.144132 & 0.528329 & 1.476797 \\ \mathrm{H} & 1.777331 & 1.284505 & 1.482231 \\ \mathrm{H} & 1.547687 & -0.241477 & 1.959964 \\ \mathrm{O} & 2.655529 & 2.847217 & 1.512221 \\ \mathrm{H} & 3.439958 & 2.992233 & 2.064217 \\ \mathrm{H} & 2.867107 & 3.169655 & 0.612425 \\ \mathrm{O} & 2.141530 & -1.752812 & 2.547291 \\ \mathrm{H} & 2.535458 & -1.810176 & 3.432043 \\ \mathrm{H} & 1.326823 & -2.318872 & 2.563668 \\ \mathrm{O} & 1.902966 & 3.649451 & -1.071077 \\ \mathrm{H} & 1.199264 & 4.154856 & -0.615532 \\ \mathrm{H} & 2.228695 & 4.205381 & -1.796542 \\ \mathrm{O} & -0.331506 & -2.998010 & 2.386398 \\ \mathrm{H} & -0.421711 & -3.566757 & 1.560668 \\ \mathrm{H} & -0.729793 & -3.491333 & 3.120200 \\ \mathrm{O} & -1.061658 & 1.898567 & 0.173985 \\ \mathrm{H} & -1.980393 & 2.055110 & -0.151655 \\ \mathrm{H} & -0.729316 & 2.700595 & 0.639546 \\ \mathrm{O} & 0.188003 & 4.160902 & 1.134094 \\ \mathrm{H} & -0.230584 & 4.925739 & 1.559317 \\ \mathrm{H} & 0.964341 & 3.916927 & 1.677812 \\ \mathrm{O} & -3.751200 & 2.002504 & -0.446603 \\ \mathrm{H} & -4.249804 & 2.780795 & -0.740476 \\ \mathrm{H} & -3.992629 & 1.264941 & -1.046407 \\ \mathrm{O} & -4.029428 & -0.690865 & -1.226153 \\ \mathrm{H} & -4.596670 & -1.220118 & -1.808597 \\ \mathrm{H} & -4.274745 & -0.912393 & -0.307050\end{array}$




\begin{tabular}{|c|c|c|c|}
\hline $\mathrm{O}$ & & & \\
\hline $\mathrm{H}$ & & & \\
\hline $\mathrm{H}$ & -4.529225 & -0.089727 & \\
\hline $\mathrm{O}$ & & & \\
\hline $\mathrm{H}$ & -2.224301 & -0.619191 & 3747 \\
\hline $\mathrm{H}$ & -0.85 & & \\
\hline $\mathrm{O}$ & & -1.4948 & \\
\hline $\mathrm{H}$ & 0.1 & -1.4 & \\
\hline $\mathrm{H}$ & 1.0 & -1.5 & \\
\hline $\mathrm{O}$ & 3.00 & -0.71 & \\
\hline $\mathrm{H}$ & & -1.2 & \\
\hline $\mathrm{H}$ & 3.7 & -0.5 & \\
\hline $\mathrm{O}$ & & & \\
\hline $\mathrm{H}$ & 1.6 & -1.7 & \\
\hline $\mathrm{O}$ & & -2.1 & \\
\hline $\mathrm{H}$ & 3.9 & -3.0 & \\
\hline $\mathrm{H}$ & & -2.0 & \\
\hline $\mathrm{O}$ & -0.6 & -3.9 & \\
\hline $\mathrm{H}$ & -0.4 & -4.7 & \\
\hline $\mathrm{H}$ & -0.1 & -3.1 & \\
\hline $\mathrm{O}$ & 1.1 & 0.9 & \\
\hline $\mathrm{H}$ & & & \\
\hline $\mathrm{H}$ & & & \\
\hline $\mathrm{O}$ & & & \\
\hline $\mathrm{H}$ & -2.2 & -0.4 & \\
\hline $\mathrm{H}$ & -1.1 & -1.5 & \\
\hline $\mathrm{CR}$ & $-0 .($ & 615 & -0 . \\
\hline \multicolumn{4}{|c|}{$\left[{ }^{4} \mathrm{Cr}\left(\mathrm{H}_{2} \mathrm{O}\right)_{5}(\mathrm{OH})\left(\mathrm{H}_{2} \mathrm{O}\right)_{12}\right]^{2+}(\mathrm{b})$} \\
\hline \multicolumn{4}{|c|}{$E=-1462.075634$} \\
\hline $\mathrm{O}$ & -1.331514 & -0.22 & \\
\hline $\mathrm{H}$ & -0.8 & -0.7 & \\
\hline $\mathrm{H}$ & & & \\
\hline $\mathrm{O}$ & & -1.4 & \\
\hline $\mathrm{H}$ & & -1.4 & \\
\hline $\mathrm{H}$ & & -1.50 & -3.1 \\
\hline $\mathrm{O}$ & -4.02 & -0.69 & \\
\hline $\mathrm{H}$ & $-4.5 \mathrm{C}$ & -1.2 & -1.8 \\
\hline $\mathrm{H}$ & -4.2 & -0.9 & -0. \\
\hline $\mathrm{O}$ & 2.99 & -0.719 & -2.3 \\
\hline $\mathrm{H}$ & 3.36 & -1.22 & -1.5 \\
\hline $\mathrm{H}$ & & -0.533229 & -2.9 \\
\hline $\mathrm{O}$ & -3.979848 & 0.006039 & 1.5 \\
\hline $\mathrm{H}$ & -4.121 & 0.910825 & \\
\hline $\mathrm{H}$ & -4.528589 & -0.090278 & 2.339139 \\
\hline $\mathrm{O}$ & 0.677122 & -1.589058 & -0.392429 \\
\hline
\end{tabular}




$\begin{array}{cccc}\mathrm{H} & 1.625504 & -1.746852 & -0.246973 \\ \mathrm{O} & -0.618765 & -3.930107 & -0.017317 \\ \mathrm{H} & -0.414738 & -4.727049 & -0.525399 \\ \mathrm{H} & -0.136439 & -3.162641 & -0.412266 \\ \mathrm{O} & 3.522634 & -2.190295 & 0.039418 \\ \mathrm{H} & 3.967765 & -3.045706 & -0.058900 \\ \mathrm{H} & 3.320654 & -2.095101 & 0.992651 \\ \mathrm{O} & 2.142423 & -1.753161 & 2.546508 \\ \mathrm{H} & 2.535855 & -1.810885 & 3.431455 \\ \mathrm{H} & 1.327747 & -2.319281 & 2.562221 \\ \mathrm{O} & -0.331010 & -2.997623 & 2.386842 \\ \mathrm{H} & -0.421893 & -3.566466 & 1.561268 \\ \mathrm{H} & -0.729043 & -3.490725 & 3.120929 \\ \mathrm{O} & 1.144815 & 0.528022 & 1.476327 \\ \mathrm{H} & 1.548633 & -0.241576 & 1.959569 \\ \mathrm{H} & 1.777566 & 1.284552 & 1.481874 \\ \mathrm{O} & 2.655608 & 2.847643 & 1.512430 \\ \mathrm{H} & 3.440117 & 2.992424 & 2.064375 \\ \mathrm{H} & 2.867285 & 3.169809 & 0.612541 \\ \mathrm{O} & 1.903557 & 3.649254 & -1.070926 \\ \mathrm{H} & 1.199991 & 4.154795 & -0.615308 \\ \mathrm{H} & 2.228674 & 4.204740 & -1.797005 \\ \mathrm{O} & -1.061507 & 1.898559 & 0.174499 \\ \mathrm{H} & -0.728660 & 2.700660 & 0.639640 \\ \mathrm{H} & -1.980359 & 2.055280 & -0.150722 \\ \mathrm{O} & -3.751129 & 2.002768 & -0.445461 \\ \mathrm{H} & -4.250297 & 2.780832 & -0.739008 \\ \mathrm{H} & -3.992184 & 1.265258 & -1.045496 \\ \mathrm{O} & 0.188488 & 4.160769 & 1.133938 \\ \mathrm{H} & -0.230573 & 4.925416 & 1.559046 \\ \mathrm{H} & 0.964769 & 3.917100 & 1.677922 \\ \mathrm{O} & 1.183823 & 0.982215 & -1.369685 \\ \mathrm{H} & 1.396003 & 1.944263 & -1.378385 \\ \mathrm{H} & 1.925041 & 0.449544 & -1.766746 \\ \mathrm{O} & -1.373629 & -0.763881 & 1.220940 \\ \mathrm{H} & -1.118824 & -1.590134 & 1.708645 \\ \mathrm{CR} & -2.272481 & -0.452272 & 1.483323 \\ & -0.089231 & 0.082631 & -0.087214\end{array}$

$\left[{ }^{4} \mathrm{Cr}\left(\mathrm{H}_{2} \mathrm{O}\right)_{5}(\mathrm{OH})\left(\mathrm{H}_{2} \mathrm{O}\right)_{12}\right]^{2+}(\mathrm{c})$

$\mathrm{E}=-1462.082388$

$\begin{array}{llll}\mathrm{O} & 0.952908 & -1.796418 & -0.023390 \\ \mathrm{H} & 0.618883 & -2.581583 & -0.547709 \\ \mathrm{H} & 1.688105 & -2.070076 & 0.578692 \\ \mathrm{O} & 2.972636 & -2.278453 & 1.755015 \\ \mathrm{H} & 3.465158 & -3.107872 & 1.855937\end{array}$




\begin{tabular}{|c|c|c|c|}
\hline H & .601756 & -1.596329 & \\
\hline ) & .007516 & -3.761677 & \\
\hline & & & \\
\hline & .281843 & -3.445005 & -2.386594 \\
\hline & .116035 & -1.992380 & \\
\hline & .814156 & -2.252819 & -4.1 \\
\hline & -0.488794 & -1.459262 & -3 \\
\hline & 0.702855 & 0.430256 & -1. \\
\hline & & & \\
\hline & 0.51 & 1.3 & -2.0 \\
\hline & & & -2 \\
\hline & 0.51 & 87167 & -3. \\
\hline & 0.05 & 3.4 & -2. \\
\hline & 3.18 & -0.303714 & -2 . \\
\hline & 3.70 & -0.251989 & -1 . \\
\hline & 3.76 & -0. & \\
\hline & -0.20 & 9720 & -0 . \\
\hline & -0.6 & & \\
\hline $\mathrm{H}$ & 0.6 & 4.0 & 0.1 \\
\hline $\mathrm{O}$ & $2.0^{\circ}$ & & \\
\hline $\mathrm{H}$ & 2.5 & & 1.5 \\
\hline & 1.8 & & \\
\hline $\mathrm{O}$ & & & \\
\hline $\mathrm{H}$ & 1.3 & 0.5 & \\
\hline $\mathrm{CR}$ & $-0 .($ & & \\
\hline $\mathrm{O}$ & -0.9 & -0.7 & \\
\hline $\mathrm{H}$ & -1.7 & -0.3 & 366 \\
\hline $\mathrm{H}$ & $-0.2^{\prime}$ & -0.9 & \\
\hline $\mathrm{O}$ & $1.1 \mathrm{C}$ & -1.0 & 3.4 \\
\hline $\mathrm{H}$ & 1.21 & 30029 & \\
\hline $\mathrm{H}$ & 1.87 & -1.5 & 3.1 \\
\hline $\mathrm{O}$ & -3.4 & & \\
\hline $\mathrm{H}$ & -3.79 & 76080 & \\
\hline $\mathrm{H}$ & -3.76 & 0.7793 & \\
\hline $\mathrm{O}$ & -3.95 & 1.380871 & \\
\hline $\mathrm{H}$ & -4.572115 & 2.060954 & -0.1 \\
\hline $\mathrm{H}$ & -4.28 & 0.521904 & -0.2 \\
\hline $\mathrm{O}$ & -4.037514 & -1.470420 & 7097 \\
\hline $\mathrm{H}$ & -4.63 & -2.208514 & 0.0 \\
\hline $\mathrm{H}$ & -4.01 & -1.358393 & \\
\hline $\mathrm{O}$ & -1.180770 & 1.607547 & 0.1 \\
\hline $\mathrm{H}$ & -2.142660 & 1.572600 & -0.013586 \\
\hline $\mathrm{H}$ & -0.854763 & 2.546418 & 0.081032 \\
\hline $\mathrm{O}$ & -1.604798 & -0.912687 & -0.944929 \\
\hline $\mathrm{H}$ & -2.411262 & -1.317210 & -0.544505 \\
\hline $\mathrm{H}$ & -1.495726 & -1.228759 & -1.871344 \\
\hline
\end{tabular}




$\begin{array}{rrrr}\mathrm{O} & 3.956635 & -0.065561 & 0.534523 \\ \mathrm{H} & 3.086809 & 0.379052 & 0.727398 \\ \mathrm{H} & 4.666155 & 0.531427 & 0.817799\end{array}$

$\left[{ }^{4} \mathrm{Cr}\left(\mathrm{H}_{2} \mathrm{O}\right)_{5}(\mathrm{OH})\left(\mathrm{H}_{2} \mathrm{O}\right)_{12}\right]^{2+}(\mathrm{d})$

$\mathrm{E}=-1462.076248$

$\begin{array}{lrrr}\mathrm{CR} & -0.283070 & -0.182459 & -0.059700 \\ \mathrm{O} & -1.406615 & 1.218890 & -0.563581 \\ \mathrm{H} & -2.291409 & 1.000633 & -0.910524 \\ \mathrm{O} & -1.639187 & -0.910479 & 1.225814 \\ \mathrm{H} & -1.296843 & -1.342530 & 2.060347 \\ \mathrm{H} & -2.365385 & -0.272108 & 1.490508 \\ \mathrm{O} & -3.640003 & 0.769887 & 1.706866 \\ \mathrm{H} & -4.308457 & 0.714094 & 2.405241 \\ \mathrm{H} & -4.110225 & 0.785079 & 0.846538 \\ \mathrm{O} & -0.642463 & -1.982169 & 3.440300 \\ \mathrm{H} & -1.068878 & -2.268269 & 4.260194 \\ \mathrm{H} & 0.302772 & -1.831465 & 3.626848 \\ \mathrm{O} & -4.254214 & 0.492789 & -1.011004 \\ \mathrm{H} & -4.903881 & 0.977115 & -1.543262 \\ \mathrm{H} & -4.317251 & -0.446821 & -1.268785 \\ \mathrm{O} & -3.657185 & -2.336258 & -1.446886 \\ \mathrm{H} & -4.016536 & -2.831414 & -2.201762 \\ \mathrm{H} & -3.868972 & -2.870272 & -0.663073 \\ \mathrm{O} & 2.173492 & -1.704967 & 3.003348 \\ \mathrm{H} & 2.749288 & -2.376715 & 3.400787 \\ \mathrm{H} & 2.700483 & -0.881184 & 2.922637 \\ \mathrm{O} & 3.202581 & 0.668852 & 1.897700 \\ \mathrm{H} & 3.767026 & 1.356290 & 2.285927 \\ \mathrm{H} & 3.607322 & 0.414455 & 1.038584 \\ \mathrm{O} & 1.102357 & 0.439601 & -1.451886 \\ \mathrm{H} & 2.051889 & 0.334199 & -1.203645 \\ \mathrm{H} & 1.007315 & 1.418972 & -1.780406 \\ \mathrm{O} & 1.157927 & 2.827745 & -2.290227 \\ \mathrm{H} & 0.584244 & 3.156748 & -2.996645 \\ \mathrm{H} & 1.168330 & 3.523391 & -1.575233 \\ \mathrm{O} & 3.763912 & -0.138401 & -0.769517 \\ \mathrm{H} & 4.504029 & 0.247789 & -1.265233 \\ \mathrm{H} & 3.723894 & -1.088640 & -1.014429 \\ \mathrm{O} & 1.146598 & 4.472467 & -0.200561 \\ \mathrm{H} & 1.342758 & 5.419849 & -0.175849 \\ \mathrm{H} & 0.350151 & 4.313184 & 0.346748 \\ \mathrm{O} & -0.928079 & 3.174447 & 1.135441 \\ \mathrm{H} & -1.628698 & 3.435348 & 1.752146 \\ \mathrm{H} & -1.342392 & 2.587915 & 0.438272 \\ \mathrm{O} & 0.507534 & 0.977784 & 1.395722 \\ & & & \end{array}$




$\begin{array}{cccc}\mathrm{H} & 1.476874 & 1.030551 & 1.572447 \\ \mathrm{H} & 0.074929 & 1.874746 & 1.496014 \\ \mathrm{O} & 2.714242 & -2.673029 & -1.484333 \\ \mathrm{H} & 3.116925 & -3.554082 & -1.541357 \\ \mathrm{H} & 2.205673 & -2.536159 & -2.313759 \\ \mathrm{O} & 0.922968 & -1.632966 & -3.418500 \\ \mathrm{H} & 1.110993 & -0.692988 & -3.231799 \\ \mathrm{H} & 0.903999 & -1.742628 & -4.382780 \\ \mathrm{O} & -0.985352 & -1.513406 & -1.434906 \\ \mathrm{H} & -1.909812 & -1.852599 & -1.459973 \\ \mathrm{H} & -0.526470 & -1.659883 & -2.290982 \\ \mathrm{O} & 1.081073 & -1.647970 & 0.518304 \\ \mathrm{H} & 1.580292 & -2.195572 & -0.128626 \\ \mathrm{H} & 1.463027 & -1.790545 & 1.420936\end{array}$

$\left[{ }^{4} \mathrm{Cr}\left(\mathrm{H}_{2} \mathrm{O}\right)_{5}(\mathrm{OH})\left(\mathrm{H}_{2} \mathrm{O}\right)_{12}\right]^{2+}(\mathrm{e})$

$\mathrm{E}=-1462.075464$

$\begin{array}{cccc}\mathrm{O} & -0.930668 & 1.243423 & 1.373797 \\ \mathrm{H} & -1.727852 & 0.767414 & 1.728366 \\ \mathrm{H} & -1.111393 & 2.211484 & 1.303169 \\ \mathrm{O} & -2.853577 & -0.343609 & 2.500854 \\ \mathrm{H} & -3.585151 & -0.055469 & 3.068310 \\ \mathrm{H} & -3.237731 & -0.967562 & 1.832978 \\ \mathrm{O} & -1.481495 & 3.885028 & 0.768632 \\ \mathrm{H} & -1.611555 & 4.613000 & 1.396279 \\ \mathrm{H} & -0.820283 & 4.184063 & 0.109828 \\ \mathrm{O} & -3.452377 & -1.944346 & 0.325522 \\ \mathrm{H} & -4.213656 & -1.755619 & -0.243286 \\ \mathrm{H} & -2.657796 & -1.476799 & -0.044814 \\ \mathrm{O} & 0.411066 & 3.858836 & -1.377130 \\ \mathrm{H} & -0.016422 & 3.177426 & -1.930893 \\ \mathrm{H} & 0.712531 & 4.566698 & -1.968423 \\ \mathrm{O} & 1.271136 & -0.514061 & 1.578406 \\ \mathrm{H} & 1.840479 & -1.316417 & 1.407726 \\ \mathrm{H} & 0.702291 & -0.725261 & 2.381499 \\ \mathrm{O} & -0.357166 & -1.259355 & 3.497643 \\ \mathrm{H} & -0.227134 & -1.389162 & 4.447553 \\ \mathrm{H} & -1.301643 & -1.044739 & 3.347609 \\ \mathrm{O} & 2.879625 & -2.553514 & 0.989900 \\ \mathrm{H} & 3.185119 & -3.297416 & 1.528851 \\ \mathrm{H} & 3.626397 & -2.245789 & 0.444115 \\ \mathrm{O} & 4.130220 & -0.735429 & -0.780978 \\ \mathrm{H} & 4.820059 & -0.844209 & -1.454357 \\ \mathrm{H} & 4.375364 & 0.047056 & -0.244020 \\ \mathrm{O} & 4.227334 & 1.540451 & 0.990097 \\ \mathrm{H} & 4.291911 & 1.275710 & 1.923066\end{array}$




$\begin{array}{cccc}\mathrm{H} & 4.876105 & 2.255705 & 0.880151 \\ \mathrm{O} & 1.411059 & -0.675998 & -1.303512 \\ \mathrm{H} & 2.395145 & -0.705909 & -1.228267 \\ \mathrm{H} & 1.061353 & -1.537364 & -1.656306 \\ \mathrm{O} & 0.099642 & -2.822505 & -2.380155 \\ \mathrm{H} & 0.490651 & -3.514841 & -2.935210 \\ \mathrm{H} & -0.507326 & -3.282461 & -1.743609 \\ \mathrm{O} & -1.533063 & -3.977928 & -0.470460 \\ \mathrm{H} & -2.388762 & -3.577644 & -0.213499 \\ \mathrm{H} & -1.597358 & -4.924820 & -0.277118 \\ \mathrm{O} & -0.733851 & 1.205855 & -1.514896 \\ \mathrm{H} & -0.840012 & 0.578313 & -2.301909 \\ \mathrm{H} & -1.661698 & 1.511911 & -1.297694 \\ \mathrm{O} & -3.103636 & 2.250019 & -0.861289 \\ \mathrm{H} & -3.878584 & 2.429100 & -1.413399 \\ \mathrm{H} & -2.935080 & 3.041642 & -0.316139 \\ \mathrm{O} & -1.173019 & -0.524448 & -3.419977 \\ \mathrm{H} & -1.205722 & -0.422780 & -4.381654 \\ \mathrm{H} & -0.820357 & -1.418613 & -3.221496 \\ \mathrm{O} & -0.984176 & -1.140663 & -0.033746 \\ \mathrm{H} & -0.665871 & -1.987844 & 0.320174 \\ \mathrm{O} & 1.565926 & 1.866551 & 0.080804 \\ \mathrm{H} & 2.462462 & 1.853350 & 0.478946 \\ \mathrm{H} & 1.422594 & 2.685612 & -0.444038 \\ \mathrm{CR} & 0.233545 & 0.267740 & 0.042059\end{array}$

$\left[{ }^{4} \mathrm{Cr}\left(\mathrm{H}_{2} \mathrm{O}\right)_{5}(\mathrm{OH})\left(\mathrm{H}_{2} \mathrm{O}\right)_{12}\right]^{2+}$ (f)

$\mathrm{E}=-1462.085469$

$\begin{array}{rrrr}\mathrm{O} & -1.764302 & 0.001950 & 0.977162 \\ \mathrm{H} & -2.429574 & -0.704704 & 1.169281 \\ \mathrm{H} & -2.168657 & 0.878429 & 1.179367 \\ \mathrm{O} & -2.806526 & 2.556474 & 1.121004 \\ \mathrm{H} & -3.435321 & 2.889942 & 1.780562 \\ \mathrm{H} & -1.996039 & 3.111062 & 1.201060 \\ \mathrm{O} & -3.630323 & -1.966621 & 1.161782 \\ \mathrm{H} & -4.461327 & -1.983187 & 1.661535 \\ \mathrm{H} & -3.866289 & -1.936797 & 0.209092 \\ \mathrm{O} & -3.580096 & -1.238302 & -1.544030 \\ \mathrm{H} & -4.059136 & -1.630218 & -2.291701 \\ \mathrm{H} & -3.692685 & -0.264357 & -1.611229 \\ \mathrm{O} & -0.205482 & 3.691916 & 1.365378 \\ \mathrm{H} & 0.221541 & 3.982637 & 0.525580 \\ \mathrm{H} & -0.019722 & 4.375941 & 2.028560 \\ \mathrm{O} & -3.310498 & 1.635932 & -1.532250 \\ \mathrm{H} & -3.777266 & 2.174729 & -2.191180 \\ \mathrm{H} & -3.417615 & 2.090534 & -0.668078\end{array}$




\begin{tabular}{|c|c|c|c|}
\hline $\mathrm{O}$ & -0.601823 & 1.581349 & \\
\hline $\mathrm{H}$ & -1.477023 & & \\
\hline $\mathrm{H}$ & -0.046599 & & \\
\hline $\mathrm{O}$ & 0.985094 & 3.762163 & \\
\hline $\mathrm{H}$ & 0.992273 & & \\
\hline $\mathrm{H}$ & 1.885960 & 40 & \\
\hline $\mathrm{O}$ & & & \\
\hline $\mathrm{H}$ & 3.79 & 807 & \\
\hline $\mathrm{H}$ & 2.60 & & \\
\hline $\mathrm{O}$ & 0.60 & -3.27 & -2 \\
\hline $\mathrm{H}$ & & & -3.1 \\
\hline $\mathrm{H}$ & 1.3 & -3.5 & -1 \\
\hline $\mathrm{O}$ & & -0.0 & \\
\hline $\mathrm{H}$ & 2.3 & -0.741 & -0.8 \\
\hline $\mathrm{CR}$ & 124 & -0.09 & -0 \\
\hline $\mathrm{O}$ & & -1.688 & \\
\hline $\mathrm{H}$ & -0.1 & -2.1 & 1. \\
\hline $\mathrm{H}$ & & -2.3 & \\
\hline $\mathrm{O}$ & 2.3 & -3.4 & \\
\hline $\mathrm{H}$ & 2.4 & -4.3 & \\
\hline $\mathrm{H}$ & 3.1 . & -2.920 & \\
\hline $\mathrm{O}$ & & & \\
\hline $\mathrm{H}$ & -1.2 & -3.5 & \\
\hline $\mathrm{H}$ & -2.1 & -3.0 & \\
\hline $\mathrm{O}$ & & -1.4 & \\
\hline $\mathrm{H}$ & 5.0 & -1.5 & -0 . \\
\hline $\mathrm{H}$ & 4.1 & -0.7 & \\
\hline $\mathrm{O}$ & 3.5 & 1.02 & \\
\hline $\mathrm{H}$ & & 1.4 & \\
\hline $\mathrm{H}$ & 3.6 & 1.59 & \\
\hline $\mathrm{O}$ & -0.7 & -1.23 & \\
\hline $\mathrm{H}$ & -0.2 & -1.9 & -1. \\
\hline $\mathrm{H}$ & -1.7 & -1.3 & 596 \\
\hline $\mathrm{O}$ & & & \\
\hline $\mathrm{H}$ & & 2.027669 & \\
\hline $\mathrm{H}$ & 1.794351 & 1.057203 & 1.58 \\
\hline \multicolumn{4}{|c|}{$\left.\left[{ }^{4} \mathrm{Cr}\left(\mathrm{H}_{2} \mathrm{O}\right)_{6}\right]\right]^{3+}$} \\
\hline \multicolumn{4}{|c|}{$\mathrm{E}=-544.2909777$} \\
\hline $\mathrm{O}$ & & & \\
\hline $\mathrm{H}$ & 0.791318 & 0.000000 & 9427 \\
\hline $\mathrm{H}$ & -0.7 & & \\
\hline $\mathrm{O}$ & 0.000000 & 2.019812 & 0.000000 \\
\hline $\mathrm{H}$ & & 2.599427 & \\
\hline $\mathrm{H}$ & 0.000000 & 2.599427 & -0.791318 \\
\hline $\mathrm{O}$ & 2.019812 & 0.000000 & 0.000000 \\
\hline
\end{tabular}




$\begin{array}{cccc}\mathrm{H} & 2.599427 & 0.791318 & 0.000000 \\ \mathrm{H} & 2.599427 & -0.791318 & 0.000000 \\ \mathrm{O} & 0.000000 & 0.000000 & -2.019812 \\ \mathrm{H} & -0.791318 & 0.000000 & -2.599427 \\ \mathrm{H} & 0.791318 & 0.000000 & -2.599427 \\ \mathrm{O} & -2.019812 & 0.000000 & 0.000000 \\ \mathrm{H} & -2.599427 & -0.791318 & 0.000000 \\ \mathrm{H} & -2.599427 & 0.791318 & 0.000000 \\ \mathrm{O} & 0.000000 & -2.019812 & 0.000000 \\ \mathrm{H} & 0.000000 & -2.599427 & 0.791318 \\ \mathrm{H} & 0.000000 & -2.599427 & -0.791318 \\ \mathrm{CR} & 0.000000 & 0.000000 & 0.000000\end{array}$

$\left[{ }^{4} \mathrm{Cr}\left(\mathrm{H}_{2} \mathrm{O}\right)_{5}(\mathrm{OH})\right]^{2+}(\mathrm{a})$

$\mathrm{E}=-544.2341496$

$\begin{array}{lrrc}\mathrm{O} & -0.150815 & 2.044658 & -0.102090 \\ \mathrm{H} & -0.371361 & 2.663223 & 0.616621 \\ \mathrm{H} & 0.357930 & 2.524476 & -0.782407 \\ \mathrm{O} & 0.900746 & 0.056371 & -1.638892 \\ \mathrm{H} & 0.500989 & -0.008779 & -2.521428 \\ \mathrm{CR} & 0.034401 & 0.006313 & -0.059271 \\ \mathrm{O} & 0.026681 & -2.041121 & -0.122227 \\ \mathrm{H} & -0.040801 & -2.650606 & 0.634536 \\ \mathrm{H} & 0.596215 & -2.458811 & -0.795500 \\ \mathrm{O} & -1.900559 & -0.086578 & -0.734936 \\ \mathrm{H} & -2.343668 & 0.664654 & -1.170360 \\ \mathrm{H} & -2.287494 & -0.907745 & -1.090623 \\ \mathrm{O} & 1.934075 & 0.034687 & 0.721360 \\ \mathrm{H} & 2.320278 & 0.473934 & 1.498534 \\ \mathrm{H} & 2.607105 & 0.025147 & 0.012214 \\ \mathrm{O} & -0.810773 & -0.043584 & 1.901180 \\ \mathrm{H} & -0.387137 & -0.160254 & 2.769653 \\ \mathrm{H} & -1.772520 & -0.032208 & 2.056103\end{array}$

$\left[{ }^{4} \mathrm{Cr}\left(\mathrm{H}_{2} \mathrm{O}\right)_{5}(\mathrm{OH})\right]^{2+}(\mathrm{b})$

$\mathrm{E}=-544.2330708$

$\begin{array}{rrrr}\mathrm{O} & 2.009206 & -0.381557 & -0.089767 \\ \mathrm{H} & 2.456365 & -0.599774 & -0.928585 \\ \mathrm{H} & 2.624243 & -0.530320 & 0.650129 \\ \mathrm{O} & 0.417435 & 2.017023 & 0.013279 \\ \mathrm{H} & 1.297223 & 2.388681 & -0.180154 \\ \mathrm{H} & -0.240287 & 2.711461 & -0.173041 \\ \mathrm{O} & -0.002761 & 0.018819 & 2.040055 \\ \mathrm{H} & -0.166428 & -0.760129 & 2.601052 \\ \mathrm{H} & 0.155956 & 0.777586 & 2.628568 \\ \mathrm{O} & -1.997293 & 0.437896 & -0.100205\end{array}$




$\begin{array}{lccc}\mathrm{H} & -2.623861 & 0.555409 & 0.635583 \\ \mathrm{H} & -2.489273 & 0.417464 & -0.942003 \\ \mathrm{O} & -0.413690 & -2.006998 & 0.120827 \\ \mathrm{H} & -1.274067 & -2.332700 & -0.203817 \\ \mathrm{H} & 0.247412 & -2.646143 & -0.204967 \\ \mathrm{CR} & 0.001157 & 0.005665 & -0.091452 \\ \mathrm{O} & -0.029072 & -0.167972 & -1.879730 \\ \mathrm{H} & 0.114341 & 0.544819 & -2.523587\end{array}$

$\begin{array}{lccc}{\left[{ }^{4} \mathrm{Cr}\left(\mathrm{H}_{2} \mathrm{O}\right)_{5}(\mathrm{OH})\right]^{2+}(\mathrm{c})} \\ \mathrm{E}=-544.2330748 \\ \mathrm{O} & 0.433517 & -2.014095 & 0.013474 \\ \mathrm{H} & 1.316918 & -2.378588 & -0.177010 \\ \mathrm{H} & -0.217842 & -2.714105 & -0.174292 \\ \mathrm{O} & 2.005537 & 0.398493 & -0.094692 \\ \mathrm{H} & 2.620698 & 0.552699 & 0.643980 \\ \mathrm{H} & 2.449933 & 0.618040 & -0.934610 \\ \mathrm{O} & 0.003546 & -0.017011 & 2.040053 \\ \mathrm{H} & 0.166814 & -0.773738 & 2.629950 \\ \mathrm{H} & -0.164124 & 0.762061 & 2.599718 \\ \mathrm{O} & -1.994191 & -0.452498 & -0.094422 \\ \mathrm{H} & -2.488124 & -0.438317 & -0.935200 \\ \mathrm{H} & -2.617393 & -0.578447 & 0.642804 \\ \mathrm{O} & -0.429027 & 2.003452 & 0.120074 \\ \mathrm{H} & -1.294774 & 2.321378 & -0.197885 \\ \mathrm{H} & 0.224187 & 2.646895 & -0.213108 \\ \mathrm{CR} & 0.000972 & -0.006054 & -0.091557 \\ \mathrm{O} & -0.036096 & 0.165744 & -1.879845 \\ \mathrm{H} & 0.114097 & -0.545256 & -2.524121\end{array}$

$\left[{ }^{4} \mathrm{Cr}\left(\mathrm{H}_{2} \mathrm{O}\right)_{5}(\mathrm{OH})\right]^{2+}(\mathrm{d})$

$\mathrm{E}=-544.2330694$

$\begin{array}{rrrc}\mathrm{O} & -0.002887 & 0.023616 & 2.039997 \\ \mathrm{H} & -0.161136 & 0.784422 & 2.625988 \\ \mathrm{H} & 0.161352 & -0.753361 & 2.603565 \\ \mathrm{O} & -0.410568 & 2.018658 & 0.007659 \\ \mathrm{H} & -1.288226 & 2.393814 & -0.188609 \\ \mathrm{H} & 0.250501 & 2.710309 & -0.177087 \\ \mathrm{O} & 1.998517 & 0.431503 & -0.095941 \\ \mathrm{H} & 2.492625 & 0.408412 & -0.936419 \\ \mathrm{H} & 2.623830 & 0.545499 & 0.641475 \\ \mathrm{O} & -2.010275 & -0.375487 & -0.094206 \\ \mathrm{H} & -2.629268 & -0.515909 & 0.644016 \\ \mathrm{H} & -2.455903 & -0.594240 & -0.933704 \\ \mathrm{O} & 0.408003 & -2.007998 & 0.126212 \\ \mathrm{H} & 1.261754 & -2.337381 & -0.212050\end{array}$




\begin{tabular}{lccc}
$\mathrm{H}$ & -0.260191 & -2.647274 & -0.184389 \\
$\mathrm{CR}$ & -0.001080 & 0.005292 & -0.091416 \\
$\mathrm{O}$ & 0.034253 & -0.172835 & -1.879215 \\
$\mathrm{H}$ & -0.105759 & 0.539049 & -2.524842 \\
\multicolumn{5}{l}{} \\
$\left.{ }^{4} \mathrm{Cr}\left(\mathrm{H}_{2} \mathrm{O}\right)_{5}(\mathrm{OH})\right]^{2+}(\mathrm{e})$ \\
$\mathrm{E}=-544.2341439$ \\
$\mathrm{O}$ & 0.127657 & -2.046924 & -0.093516 \\
$\mathrm{H}$ & 0.343303 & -2.665807 & 0.626390 \\
$\mathrm{H}$ & -0.386739 & -2.523417 & -0.771906 \\
$\mathrm{O}$ & 0.810515 & 0.044787 & 1.901268 \\
$\mathrm{H}$ & 0.387706 & 0.164795 & 2.769675 \\
$\mathrm{H}$ & 1.772113 & 0.025509 & 2.056321 \\
$\mathrm{O}$ & 1.901675 & 0.061623 & -0.734554 \\
$\mathrm{H}$ & 2.298324 & 0.876531 & -1.093916 \\
$\mathrm{H}$ & 2.335743 & -0.696734 & -1.166699 \\
$\mathrm{O}$ & -0.003359 & 2.040772 & -0.130714 \\
$\mathrm{H}$ & -0.567115 & 2.462281 & -0.806475 \\
$\mathrm{H}$ & 0.067448 & 2.651212 & 0.624996 \\
$\mathrm{O}$ & -1.934606 & -0.011013 & 0.720589 \\
$\mathrm{H}$ & -2.326406 & -0.441917 & 1.499626 \\
$\mathrm{H}$ & -2.607065 & 0.003063 & 0.010964 \\
$\mathrm{CR}$ & -0.034517 & -0.006117 & -0.059149 \\
$\mathrm{O}$ & -0.900537 & -0.053373 & -1.639049 \\
$\mathrm{H}$ & -0.499655 & 0.004332 & -2.521585
\end{tabular}

$\begin{array}{cccc}{\left[{ }^{4} \mathrm{Cr}\left(\mathrm{H}_{2} \mathrm{O}\right)_{5}(\mathrm{OH})\right]^{2+}(\mathrm{f})} \\ \mathrm{E}=-544.2330691 \\ \mathrm{O} & 0.403256 & 2.008931 & 0.124663 \\ \mathrm{H} & 1.258035 & 2.339877 & -0.209421 \\ \mathrm{H} & -0.264734 & 2.646240 & -0.190385 \\ \mathrm{O} & 1.999789 & -0.427566 & -0.090266 \\ \mathrm{H} & 2.494902 & -0.403358 & -0.930125 \\ \mathrm{H} & 2.624368 & -0.539182 & 0.648123 \\ \mathrm{O} & -0.009129 & -0.021020 & 2.039917 \\ \mathrm{H} & -0.165254 & -0.782201 & 2.626005 \\ \mathrm{H} & 0.151713 & 0.756748 & 2.603368 \\ \mathrm{O} & -0.406194 & -2.019363 & 0.008638 \\ \mathrm{H} & -1.283309 & -2.396004 & -0.187174 \\ \mathrm{H} & 0.256194 & -2.709595 & -0.176725 \\ \mathrm{O} & -2.010913 & 0.370722 & -0.099765 \\ \mathrm{H} & -2.631448 & 0.513429 & 0.636721 \\ \mathrm{H} & -2.454285 & 0.588220 & -0.940778 \\ \mathrm{CR} & -0.000752 & -0.005396 & -0.091155 \\ \mathrm{O} & 0.039619 & 0.170432 & -1.879104 \\ \mathrm{H} & -0.099550 & -0.541762 & -2.524555\end{array}$




\begin{tabular}{|c|c|c|c|}
\hline \multicolumn{4}{|c|}{$\left[{ }^{4} \mathrm{Cr}\left(\mathrm{H}_{2} \mathrm{O}\right)_{5}\right]^{3}$} \\
\hline CR & 0.000379 & 0.000001 & -0.147376 \\
\hline $\mathrm{O}$ & -0.002133 & 1.964061 & -0.439852 \\
\hline $\mathrm{H}$ & 0.788829 & 2.542854 & -0.529364 \\
\hline $\mathrm{O}$ & -2.001781 & -0.003020 & -0.318762 \\
\hline $\mathrm{H}$ & -2.652461 & -0.004046 & 0.419094 \\
\hline $\mathrm{O}$ & 0.003262 & -1.964471 & -0.4 \\
\hline $\mathrm{H}$ & 0.795671 & -2.541 & -0.5 \\
\hline $\mathrm{O}$ & 2.002790 & 0.0 & 49 \\
\hline $\mathrm{H}$ & 2.652590 & 0.00 & \\
\hline $\mathrm{O}$ & -0.003642 & & \\
\hline $\mathrm{H}$ & -0.001903 & 0.79 & \\
\hline $\mathrm{H}$ & -0.794837 & & \\
\hline $\mathrm{H}$ & -2.519492 & -0.0 & -1. \\
\hline $\mathrm{H}$ & -0.787951 & -2.5 & \\
\hline $\mathrm{H}$ & 2.521577 & -0.0 & \\
\hline $\mathrm{H}$ & 0.000911 & -0.7 & \\
\hline \multicolumn{4}{|c|}{$\left[{ }^{4} \mathrm{Cr}\left(\mathrm{H}_{2} \mathrm{O}\right)_{4}(\mathrm{OH})\right]^{2+}$} \\
\hline \multicolumn{4}{|c|}{$E=-467.7183482$} \\
\hline $\mathrm{CR}$ & -0.020010 & 500 & \\
\hline $\mathrm{O}$ & -0.3995 & & \\
\hline $\mathrm{H}$ & 0.087550 & 2.7 & -0. \\
\hline $\mathrm{O}$ & 2.01 & & \\
\hline $\mathrm{H}$ & 2.639125 & -0.0 & -0 . \\
\hline $\mathrm{H}$ & 2.466136 & 1.2 & \\
\hline $\mathrm{O}$ & -2.050650 & -0.0 & \\
\hline $\mathrm{H}$ & & & \\
\hline $\mathrm{H}$ & -2.592645 & & \\
\hline $\mathrm{O}$ & 0.119400 & -1.1 & \\
\hline $\mathrm{H}$ & -0.639655 & & \\
\hline $\mathrm{H}$ & 0.932331 & -1.3 & \\
\hline $\mathrm{O}$ & 0.274221 & -1.3 & -1.2 \\
\hline $\mathrm{H}$ & 0.374313 & -2.3 & -1. \\
\hline $\mathrm{H}$ & 0.172661 & -1.349319 & -2.3 \\
\hline \multicolumn{4}{|c|}{$\left[{ }^{4} \mathrm{Cr}\left(\mathrm{H}_{2} \mathrm{O}\right)_{4}\right]^{3+}$} \\
\hline \multicolumn{4}{|c|}{$E=-391.1646418$} \\
\hline $\mathrm{O}$ & -0.001441 & -1.57 & -0.8 \\
\hline $\mathrm{O}$ & 1.916811 & 0.06 & \\
\hline $\mathrm{O}$ & 0.001404 & 1.49 & -0.95 \\
\hline $\mathrm{O}$ & -1.916713 & 0.063590 & 0.687274 \\
\hline $\mathrm{H}$ & & -2.019188 & -1.275436 \\
\hline $\mathrm{H}$ & 2.372901 & -0.445200 & 1.406804 \\
\hline
\end{tabular}




$\begin{array}{lccc}\mathrm{H} & 0.000447 & 1.465553 & -1.948629 \\ \mathrm{H} & -2.374019 & -0.443504 & 1.405784 \\ \mathrm{H} & -0.802718 & -2.016905 & -1.276093 \\ \mathrm{H} & 2.613160 & 0.608162 & 0.246776 \\ \mathrm{H} & 0.002585 & 2.451343 & -0.686196 \\ \mathrm{H} & -2.611831 & 0.612723 & 0.247900 \\ \mathrm{CR} & 0.000004 & -0.025858 & 0.239378\end{array}$

$\begin{array}{cccc}{\left[{ }^{4} \mathrm{Cr}\left(\mathrm{H}_{2} \mathrm{O}\right)_{3}(\mathrm{OH})\right]^{2+}} \\ \mathrm{E}=-391.1911876 \\ \mathrm{O} & 0.257661 & -1.605529 & 0.663132 \\ \mathrm{O} & -2.018936 & -0.168847 & -0.425132 \\ \mathrm{O} & -0.096460 & 1.817029 & 0.474615 \\ \mathrm{O} & 1.985870 & 0.033033 & -0.439265 \\ \mathrm{H} & -0.332327 & -2.223773 & 1.143749 \\ \mathrm{H} & -2.434199 & -0.560009 & -1.221277 \\ \mathrm{H} & 0.618399 & 2.275885 & 0.962062 \\ \mathrm{H} & 2.560843 & -0.710272 & -0.163845 \\ \mathrm{H} & -2.718473 & -0.116576 & 0.256883 \\ \mathrm{H} & -0.876675 & 2.407752 & 0.468109 \\ \mathrm{H} & 2.502889 & 0.611271 & -1.032961 \\ \mathrm{CR} & -0.014397 & -0.095406 & -0.108313\end{array}$

$\left[{ }^{4} \mathrm{Cr}\left(\mathrm{H}_{2} \mathrm{O}\right)_{3}\right]^{3+}$

$\mathrm{E}=-314.573911$

$\begin{array}{lrcc}\mathrm{O} & 1.697364 & 0.750754 & -0.167552 \\ \mathrm{H} & 2.219056 & 1.361747 & 0.430830 \\ \mathrm{H} & 2.200310 & 0.688163 & -1.029081 \\ \mathrm{O} & -0.197972 & -1.844770 & -0.167322 \\ \mathrm{H} & -0.503593 & -2.249409 & -1.028696 \\ \mathrm{H} & 0.070270 & -2.601796 & 0.431425 \\ \mathrm{O} & -1.499657 & 1.093311 & -0.167480 \\ \mathrm{H} & -1.698056 & 1.559361 & -1.029202 \\ \mathrm{H} & -2.289249 & 1.239462 & 0.431443 \\ \mathrm{CR} & 0.000141 & 0.000338 & 0.242172\end{array}$

$\left[{ }^{4} \mathrm{Cr}\left(\mathrm{H}_{2} \mathrm{O}\right)_{2}(\mathrm{OH})\right]^{2+}$

$\mathrm{E}=-314.652223$

$\begin{array}{rrrr}\mathrm{O} & 0.000654 & -1.810522 & -0.125195 \\ \mathrm{H} & 0.000968 & -2.466329 & -0.863375 \\ \mathrm{O} & -1.595780 & 0.982473 & -0.090800 \\ \mathrm{H} & -1.779432 & 1.616886 & -0.817205 \\ \mathrm{H} & -2.423529 & 0.902529 & 0.434298 \\ \mathrm{O} & 1.594989 & 0.983774 & -0.090909 \\ \mathrm{H} & 1.774357 & 1.624642 & -0.812680 \\ \mathrm{H} & 2.425237 & 0.900440 & 0.429675\end{array}$




$$
\begin{array}{lccc}
\mathrm{CR} & 0.000146 & -0.159332 & 0.170189 \\
\multicolumn{4}{c}{} \\
{\left[{ }^{4} \mathrm{Cr}\left(\mathrm{H}_{2} \mathrm{O}\right)_{2}\right]^{3+}} \\
\mathrm{E}=-237.9513887 \\
\mathrm{O} & -1.903893 & 0.000042 & -0.000196 \\
\mathrm{H} & -2.515182 & -0.810420 & 0.000584 \\
\mathrm{H} & -2.516056 & 0.809944 & 0.001012 \\
\mathrm{O} & 1.904570 & 0.000024 & -0.000239 \\
\mathrm{H} & 2.515987 & -0.810419 & 0.001144 \\
\mathrm{H} & 2.516424 & 0.810189 & 0.000716 \\
\mathrm{CR} & -0.000274 & 0.000008 & 0.000001
\end{array}
$$

$$
\begin{aligned}
& {\left[{ }^{4} \mathrm{Cr}\left(\mathrm{H}_{2} \mathrm{O}\right)(\mathrm{OH})\right]^{2+}} \\
& \mathrm{E}=-238.0833427
\end{aligned}
$$

$\begin{array}{lrlc}\mathrm{O} & 1.617536 & 0.427078 & -0.037349 \\ \mathrm{H} & 2.268960 & 0.960400 & 0.494445 \\ \mathrm{O} & -1.718926 & 0.316711 & 0.015642 \\ \mathrm{H} & -2.418191 & 0.110249 & 0.682043 \\ \mathrm{H} & -2.104646 & 0.979754 & -0.606489 \\ \mathrm{CR} & 0.127708 & -0.333363 & -0.016514\end{array}$

$$
\begin{array}{cccc}
{\left[{ }^{4} \mathrm{Cr}\left(\mathrm{H}_{2} \mathrm{O}\right)\right]^{3+}} & & \\
\mathrm{E}=-161.2462354 & & \\
\mathrm{CR} & 0.000000 & 0.000000 & 0.580373 \\
\mathrm{O} & 0.000000 & 0.000000 & -1.272614 \\
\mathrm{H} & 0.000000 & -0.843973 & -1.874017 \\
\mathrm{H} & 0.000000 & 0.843973 & -1.874017
\end{array}
$$

$\left[{ }^{4} \mathrm{Cr}(\mathrm{OH})\right]^{2+}$

$\mathrm{E}=-161.4943808$

$$
\begin{array}{lccc}
\mathrm{CR} & 0.018885 & -0.486821 & 0.000000 \\
\mathrm{O} & 0.018885 & 1.210037 & 0.000000 \\
\mathrm{H} & -0.604334 & 2.003405 & 0.000000
\end{array}
$$

$\left[\left(\mathrm{NH}_{3}{ }^{+} \mathrm{CH}_{2} \mathrm{COO}^{-}\right){ }^{4} \mathrm{Cr}\left(\mathrm{H}_{2} \mathrm{O}\right)_{4}\right]^{3+}$

$$
\begin{array}{cccc}
\mathrm{E}= & -675.9684581 & & \\
\mathrm{C} & 2.977106 & -0.004197 & -0.635472 \\
\mathrm{H} & 3.213284 & 0.874215 & -1.245114 \\
\mathrm{H} & 3.213284 & -0.890336 & -1.233877 \\
\mathrm{C} & 1.490793 & -0.002290 & -0.322982 \\
\mathrm{O} & 0.636426 & -0.004606 & -1.275857 \\
\mathrm{O} & 0.995958 & 0.001445 & 0.864945 \\
\mathrm{CR} & -0.846606 & 0.000111 & 0.026448 \\
\mathrm{~N} & 3.860770 & 0.003573 & 0.590022 \\
\mathrm{H} & 3.724356 & -0.822666 & 1.188364 \\
\mathrm{H} & 4.851807 & 0.002364 & 0.300320
\end{array}
$$




\begin{tabular}{|c|c|c|c|}
\hline $\mathrm{O}$ & -0.930714 & 2.030532 & 0.100569 \\
\hline $\mathrm{O}$ & -0.933634 & -2.029699 & 0.111875 \\
\hline $\mathrm{O}$ & -2.195602 & 0.005559 & 1.570851 \\
\hline $\mathrm{H}$ & -2.537602 & 0.793582 & 2.036332 \\
\hline $\mathrm{O}$ & -2.330747 & -0.002900 & -1.376279 \\
\hline $\mathrm{H}$ & -3.295371 & -0.001632 & -1.216045 \\
\hline $\mathrm{H}$ & -2.187983 & -0.005763 & -2.344396 \\
\hline $\mathrm{H}$ & -0.471567 & 2.616727 & 0.732256 \\
\hline $\mathrm{H}$ & -1.332016 & 2.593477 & -0.590829 \\
\hline $\mathrm{H}$ & -2.538028 & -0.779248 & 2.041434 \\
\hline $\mathrm{H}$ & -1.336683 & -2.595723 & -0.575991 \\
\hline $\mathrm{H}$ & -0.474733 & -2.613223 & 0.746210 \\
\hline $\mathrm{H}$ & 3.723524 & 0.836841 & 1.178333 \\
\hline \multicolumn{4}{|c|}{$\begin{array}{l}{\left[\left(\mathrm{NH}_{3}{ }^{+} \mathrm{CH}_{2} \mathrm{COO}^{-}\right){ }^{4} \mathrm{Cr}\left(\mathrm{H}_{2} \mathrm{O}\right)_{3}\left(\mathrm{OH}^{-}\right)\right]^{2+}(\mathrm{a})} \\
\mathrm{E}=-675.863\end{array}$} \\
\hline $\mathrm{C}$ & 1.498693 & -0.288719 & -0.266319 \\
\hline $\mathrm{C}$ & 2.994448 & -0.420903 & -0.509024 \\
\hline $\mathrm{O}$ & 1.066820 & 0.202322 & 0.829125 \\
\hline $\mathrm{N}$ & 3.738651 & 0.437211 & 0.490337 \\
\hline $\mathrm{H}$ & 3.300248 & -1.461156 & -0.371251 \\
\hline $\mathrm{H}$ & 3.254783 & -0.108981 & -1.521416 \\
\hline $\mathrm{O}$ & 0.662778 & -0.658918 & -1.149655 \\
\hline $\mathrm{H}$ & 3.287464 & 0.372647 & 1.414097 \\
\hline $\mathrm{H}$ & 4.721792 & 0.151909 & 0.575665 \\
\hline $\mathrm{H}$ & 3.723237 & 1.428755 & 0.221718 \\
\hline $\mathrm{O}$ & -2.411258 & -0.576475 & -1.200560 \\
\hline $\mathrm{H}$ & -3.210070 & -0.021571 & -1.261374 \\
\hline $\mathrm{H}$ & -2.255612 & -0.954638 & -2.084646 \\
\hline $\mathrm{O}$ & -0.759460 & 1.755272 & -0.719479 \\
\hline $\mathrm{H}$ & -0.626171 & 1.936949 & -1.662470 \\
\hline $\mathrm{O}$ & -2.167771 & 0.971502 & 1.276640 \\
\hline $\mathrm{H}$ & -2.452611 & 0.727046 & 2.172516 \\
\hline $\mathrm{H}$ & -2.189422 & 1.942140 & 1.179286 \\
\hline $\mathrm{O}$ & -1.143192 & -1.735750 & 1.049776 \\
\hline $\mathrm{H}$ & -0.615804 & -2.105300 & 1.776281 \\
\hline $\mathrm{H}$ & -1.815987 & -2.394794 & 0.808047 \\
\hline CR & -0.843107 & 0.084192 & -0.029730 \\
\hline \multicolumn{4}{|c|}{$\left[\left(\mathrm{NH}_{3}{ }^{+} \mathrm{CH}_{2} \mathrm{COO}^{-}\right){ }^{4} \mathrm{Cr}\left(\mathrm{H}_{2} \mathrm{O}\right)_{3}\left(\mathrm{OH}^{-}\right)\right]^{2+}(\mathrm{b})$} \\
\hline \multicolumn{4}{|c|}{$E=-675.864$} \\
\hline $\mathrm{C}$ & 1.474613 & -0.058266 & 0.417153 \\
\hline $\mathrm{C}$ & 2.973108 & -0.110926 & 0.673295 \\
\hline $\mathrm{O}$ & 1.036836 & 0.001412 & -0.785395 \\
\hline $\mathrm{N}$ & 3.727332 & 0.106446 & -0.620264 \\
\hline $\mathrm{H}$ & 3.271786 & 0.650329 & 1.396217 \\
\hline
\end{tabular}




\begin{tabular}{|c|c|c|c|}
\hline $\mathrm{H}$ & 3.242119 & -1.092709 & 1.070295 \\
\hline $\mathrm{O}$ & 0.651605 & -0.092723 & 1.378144 \\
\hline $\mathrm{H}$ & 3.691551 & 1.088158 & -0.919909 \\
\hline $\mathrm{H}$ & 4.716178 & -0.155936 & -0.524317 \\
\hline $\mathrm{H}$ & 3.308761 & -0.448849 & -1.378785 \\
\hline $\mathrm{O}$ & -2.630271 & 0.037127 & 1.042108 \\
\hline $\mathrm{H}$ & -3.457123 & 0.040131 & 0.525858 \\
\hline $\mathrm{H}$ & -2.830014 & -0.162298 & 1.971523 \\
\hline $\mathrm{O}$ & -0.907266 & -2.051083 & -0.161309 \\
\hline $\mathrm{H}$ & -1.520788 & -2.416191 & -0.824544 \\
\hline $\mathrm{H}$ & -0.864080 & -2.666790 & 0.589682 \\
\hline $\mathrm{O}$ & -0.811273 & 2.054056 & 0.017496 \\
\hline $\mathrm{H}$ & -1.386257 & 2.479390 & -0.645306 \\
\hline $\mathrm{H}$ & -0.894945 & 2.554190 & 0.847759 \\
\hline $\mathrm{CR}$ & -0.884774 & 0.001176 & -0.070659 \\
\hline $\mathrm{O}$ & -1.981522 & 0.084822 & -1.513141 \\
\hline $\mathrm{H}$ & -1.685143 & 0.103472 & -2.436718 \\
\hline \multicolumn{4}{|c|}{$\begin{array}{l}{\left[\left(\mathrm{NH}_{3}{ }^{+} \mathrm{CH}_{2} \mathrm{COO}^{-}\right){ }^{4} \mathrm{Cr}\left(\mathrm{H}_{2} \mathrm{O}\right)_{3}\left(\mathrm{OH}^{-}\right)\right]^{2+}(\mathrm{c})} \\
\mathrm{E}=-675.862\end{array}$} \\
\hline $\mathrm{C}$ & 1.505968 & 0.044848 & 0.316428 \\
\hline $\mathrm{C}$ & 2.995619 & -0.077857 & 0.608021 \\
\hline $\mathrm{O}$ & 1.083448 & 0.082642 & -0.885284 \\
\hline $\mathrm{N}$ & 3.768846 & 0.062927 & -0.686880 \\
\hline $\mathrm{H}$ & 3.323823 & 0.677699 & 1.323254 \\
\hline $\mathrm{H}$ & 3.193386 & -1.064923 & 1.032089 \\
\hline $\mathrm{O}$ & 0.675477 & 0.068597 & 1.278979 \\
\hline $\mathrm{H}$ & 4.091726 & 1.024983 & -0.840576 \\
\hline $\mathrm{H}$ & 4.594996 & -0.546116 & -0.709908 \\
\hline $\mathrm{H}$ & 3.143592 & -0.180539 & -1.472483 \\
\hline $\mathrm{O}$ & -0.745787 & -2.045164 & 0.028505 \\
\hline $\mathrm{H}$ & -0.919913 & -2.538460 & -0.792684 \\
\hline $\mathrm{H}$ & -1.262974 & -2.477529 & 0.733315 \\
\hline $\mathrm{O}$ & -2.293944 & -0.197167 & -1.495428 \\
\hline $\mathrm{H}$ & -2.318188 & 0.287096 & -2.338498 \\
\hline $\mathrm{H}$ & -3.205092 & -0.237534 & -1.146532 \\
\hline $\mathrm{O}$ & -1.093781 & 2.050997 & -0.056199 \\
\hline $\mathrm{H}$ & -0.471275 & 2.754689 & -0.299320 \\
\hline $\mathrm{H}$ & -1.794913 & 2.427322 & 0.506141 \\
\hline $\mathrm{CR}$ & -0.907605 & 0.011286 & 0.042670 \\
\hline $\mathrm{O}$ & -2.340221 & -0.037149 & 1.145531 \\
\hline $\mathrm{H}$ & -2.265623 & -0.022035 & 2.113761 \\
\hline \multicolumn{4}{|c|}{$\begin{array}{l}{\left[\left(\mathrm{NH}_{3}{ }^{+} \mathrm{CH}_{2} \mathrm{COO}^{-}\right){ }^{4} \mathrm{Cr}\left(\mathrm{H}_{2} \mathrm{O}\right)_{3}\left(\mathrm{OH}^{-}\right)\right]^{2+}(\mathrm{d})} \\
\mathrm{E}=-675.863\end{array}$} \\
\hline $\mathrm{C}$ & -1.498709 & -0.288446 & -0.266574 \\
\hline
\end{tabular}




\begin{tabular}{|c|c|c|c|}
\hline $\mathrm{C}$ & .994460 & & \\
\hline $\mathrm{O}$ & & & \\
\hline $\mathrm{N}$ & -3.738752 & & \\
\hline $\mathrm{H}$ & -3.254844 & -0.107601 & -1.521375 \\
\hline $\mathrm{H}$ & -3.300215 & -1.460900 & 72557 \\
\hline $\mathrm{O}$ & -0.662877 & -0.6 & \\
\hline $\mathrm{H}$ & -3.722723 & & \\
\hline $\mathrm{H}$ & -4.722079 & & \\
\hline $\mathrm{H}$ & -3.288 & & \\
\hline $\mathrm{O}$ & 65 & -0.5 & \\
\hline $\mathrm{H}$ & 58 & & \\
\hline $\mathrm{H}$ & 2.2 & -0. & -2 . \\
\hline $\mathrm{O}$ & 1.1 & -1 . & \\
\hline $\mathrm{H}$ & 0.61 & & \\
\hline $\mathrm{H}$ & 1.81 & -2 . & \\
\hline $\mathrm{O}$ & & & \\
\hline $\mathrm{H}$ & 2.1 & & \\
\hline $\mathrm{H}$ & 2.4 & & \\
\hline CR & & & -0 \\
\hline $\mathrm{O}$ & 0.7 & & \\
\hline $\mathrm{H}$ & 0.6 & & \\
\hline \multirow{2}{*}{\multicolumn{4}{|c|}{$\begin{array}{l}{\left[\left(\mathrm{NH}_{2} \mathrm{CH}_{2} \mathrm{COO}^{-}\right){ }^{4} \mathrm{Cr}\left(\mathrm{H}_{2} \mathrm{O}\right)_{3}(\mathrm{OH})\right]^{+}(\mathrm{a})} \\
\mathrm{E}=-675.6102117\end{array}$}} \\
\hline & & & \\
\hline $\mathrm{C}$ & -1.539466 & & \\
\hline & -3.0 & & -0 . \\
\hline $\mathrm{O}$ & $-1 .($ & & \\
\hline & & -0 . & \\
\hline & -3.2 & & 797 \\
\hline & & & 110 \\
\hline 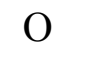 & -0.6 & & \\
\hline & & & \\
\hline & -3.76 & -0 . & \\
\hline ) & & & \\
\hline & 0.46 & -2.5 & \\
\hline $\mathrm{R}$ & 0.784821 & & 0. \\
\hline & 2.126093 & -0.2 & 639 \\
\hline & 2.130 & & \\
\hline & 2.153867 & -1.2 & 027 \\
\hline $\mathrm{O}$ & 2.383371 & & -1.3 \\
\hline & 2.115268 & & -2.2 \\
\hline & 3.010542 & -0.5 & -1.2 \\
\hline $\mathrm{O}$ & 0.898293 & & 0.331448 \\
\hline & & & 0.627444 \\
\hline $\mathrm{H}$ & 1.528057 & 2.614460 & -0.132485 \\
\hline
\end{tabular}


$\left[\left(\mathrm{NH}_{2} \mathrm{CH}_{2} \mathrm{COO}^{-}\right){ }^{4} \mathrm{Cr}\left(\mathrm{H}_{2} \mathrm{O}\right)_{3}(\mathrm{OH})\right]^{+}(\mathrm{b})$

$\mathrm{E}=-675.6099748$

$\begin{array}{cccc}\mathrm{C} & 1.519057 & -0.002084 & -0.351037 \\ \mathrm{C} & 3.017468 & -0.055473 & -0.587078 \\ \mathrm{O} & 1.005072 & 0.143950 & 0.825396 \\ \mathrm{~N} & 3.881795 & 0.050945 & 0.566077 \\ \mathrm{H} & 3.209181 & -0.992917 & -1.123445 \\ \mathrm{H} & 3.245591 & 0.735457 & -1.312319 \\ \mathrm{O} & 0.668919 & -0.102397 & -1.315837 \\ \mathrm{H} & 3.773892 & 0.934077 & 1.054389 \\ \mathrm{H} & 3.736998 & -0.703089 & 1.230003 \\ \mathrm{O} & -0.757858 & -2.047517 & 0.220773 \\ \mathrm{H} & -0.673084 & -2.608488 & -0.567387 \\ \mathrm{H} & -1.440003 & -2.426352 & 0.800412 \\ \mathrm{O} & -2.034951 & 0.115300 & 1.458772 \\ \mathrm{H} & -1.776764 & 0.063207 & 2.390556 \\ \mathrm{CR} & -0.814391 & 0.003446 & 0.070785 \\ \mathrm{O} & -0.986003 & 2.050244 & -0.126618 \\ \mathrm{H} & -0.250151 & 2.640444 & -0.356358 \\ \mathrm{H} & -1.442192 & 2.409973 & 0.655163 \\ \mathrm{O} & -2.546789 & -0.203139 & -1.103660 \\ \mathrm{H} & -2.693304 & 0.263723 & -1.942135 \\ \mathrm{H} & -3.323615 & -0.061541 & -0.532168\end{array}$

$\left[\left(\mathrm{NH}_{2} \mathrm{CH}_{2} \mathrm{COO}^{-}\right){ }^{4} \mathrm{Cr}\left(\mathrm{H}_{2} \mathrm{O}\right)_{3}(\mathrm{OH})\right]^{+}(\mathrm{c})$

$\mathrm{E}=-675.6099532$

$\begin{array}{cccc}\mathrm{C} & -1.530899 & 0.070930 & -0.280049 \\ \mathrm{C} & -3.032305 & 0.067048 & -0.504284 \\ \mathrm{O} & -0.987036 & -0.069411 & 0.877695 \\ \mathrm{~N} & -3.878564 & -0.068840 & 0.659035 \\ \mathrm{H} & -3.269849 & 0.990097 & -1.047128 \\ \mathrm{H} & -3.232998 & -0.739913 & -1.219724 \\ \mathrm{O} & -0.705137 & 0.215563 & -1.266828 \\ \mathrm{H} & -3.733588 & -0.948412 & 1.144258 \\ \mathrm{H} & -3.753809 & 0.688604 & 1.323037 \\ \mathrm{O} & 0.754473 & -2.045853 & -0.253484 \\ \mathrm{H} & 0.471426 & -2.618830 & 0.477418 \\ \mathrm{H} & 1.544826 & -2.434374 & -0.665004 \\ \mathrm{O} & 2.376169 & 0.074490 & -1.073694 \\ \mathrm{H} & 2.360850 & 0.054972 & -2.041866 \\ \mathrm{CR} & 0.830240 & 0.002156 & -0.058980 \\ \mathrm{O} & 1.009423 & 2.041645 & 0.207539 \\ \mathrm{H} & 0.255145 & 2.653015 & 0.190253 \\ \mathrm{H} & 1.687981 & 2.374295 & -0.407461 \\ \mathrm{O} & 2.184100 & -0.271444 & 1.531971 \\ \mathrm{H} & 2.127812 & 0.192549 & 2.382811\end{array}$




\begin{tabular}{cccc}
$\mathrm{H}$ & 3.089662 & -0.169652 & 1.186082 \\
\multicolumn{4}{c}{} \\
{$\left[\left(\mathrm{NH}_{2} \mathrm{CH}_{2} \mathrm{COO}^{-}\right)\right.$} & $\left.{ }^{4} \mathrm{Cr}\left(\mathrm{H}_{2} \mathrm{O}\right)_{3}(\mathrm{OH})\right]^{+}(\mathrm{d})$ \\
$\mathrm{E}=-675.6102117$ & & \\
$\mathrm{C}$ & 1.539466 & 0.006930 & -0.325766 \\
$\mathrm{C}$ & 3.037312 & 0.016022 & -0.567462 \\
$\mathrm{O}$ & 1.016217 & -0.044140 & 0.851252 \\
$\mathrm{~N}$ & 3.897469 & -0.096369 & 0.587658 \\
$\mathrm{H}$ & 3.238410 & -0.796696 & -1.275798 \\
$\mathrm{H}$ & 3.257119 & 0.936411 & -1.123108 \\
$\mathrm{O}$ & 0.689343 & 0.046977 & -1.299774 \\
$\mathrm{H}$ & 3.775841 & 0.668188 & 1.243746 \\
$\mathrm{H}$ & 3.767992 & -0.972076 & 1.084385 \\
$\mathrm{O}$ & -1.096519 & -1.882284 & -0.070614 \\
$\mathrm{H}$ & -0.465330 & -2.500831 & -0.464987 \\
$\mathrm{CR}$ & -0.784821 & -0.079660 & 0.003770 \\
$\mathrm{O}$ & -2.126093 & -0.258576 & 1.599639 \\
$\mathrm{H}$ & -2.130539 & 0.257214 & 2.420848 \\
$\mathrm{H}$ & -2.153869 & -1.209479 & 1.817025 \\
$\mathrm{O}$ & -2.383369 & 0.235146 & -1.346909 \\
$\mathrm{H}$ & -2.115264 & 0.274278 & -2.281491 \\
$\mathrm{H}$ & -3.010537 & -0.507751 & -1.280625 \\
$\mathrm{O}$ & -0.898295 & 2.040204 & 0.331448 \\
$\mathrm{H}$ & -0.153273 & 2.586389 & 0.627445 \\
$\mathrm{H}$ & -1.528061 & 2.614459 & -0.132484
\end{tabular}

Geometries of calculated acidities of $\mathrm{Cr}$ (III) complexed to acids (Table 3). $\mathrm{CH}_{3} \mathrm{COOH}$

$\begin{array}{crrr}\mathrm{E}= & -229.1564808 & & \\ \mathrm{C} & 0.000000 & 0.153428 & 0.000000 \\ \mathrm{O} & 0.173554 & 1.358879 & 0.000000 \\ \mathrm{O} & -1.248088 & -0.402779 & 0.000000 \\ \mathrm{H} & -1.878625 & 0.339735 & 0.000000 \\ \mathrm{C} & 1.079459 & -0.901566 & 0.000000 \\ \mathrm{H} & 0.970831 & -1.539286 & 0.881968 \\ \mathrm{H} & 0.970831 & -1.539286 & -0.881968 \\ \mathrm{H} & 2.056481 & -0.421141 & 0.000000\end{array}$

$\mathrm{CH}_{3} \mathrm{COO}^{-}$

$\mathrm{E}=-228.5894085$

$\begin{array}{cccc}\mathrm{C} & 0.000000 & 0.209475 & 0.000000 \\ \mathrm{O} & 1.123051 & 0.798018 & 0.000000 \\ \mathrm{O} & -1.164736 & 0.712409 & 0.000000 \\ \mathrm{C} & 0.040791 & -1.356567 & 0.000000 \\ \mathrm{H} & -0.490469 & -1.734915 & 0.882416 \\ \mathrm{H} & -0.490469 & -1.734915 & -0.882416\end{array}$




$$
\begin{array}{cccc}
\mathrm{H} & 1.069679 & -1.731036 & 0.000000 \\
\multicolumn{4}{c}{} \\
{\left[\left(\mathrm{CH}_{3} \mathrm{COO}^{-}\right)^{4} \mathrm{Cr}\left(\mathrm{H}_{2} \mathrm{O}\right)_{4}\right]^{2+}} \\
\mathrm{E}=-620.4375391 \\
\mathrm{CR} & -0.536138 & -0.000113 & 0.005903 \\
\mathrm{O} & -2.056469 & -0.001315 & 1.357076 \\
\mathrm{O} & -0.598389 & 2.026056 & 0.081092 \\
\mathrm{H} & -2.440918 & -0.788658 & 1.791681 \\
\mathrm{H} & -2.429817 & 0.785414 & 1.802380 \\
\mathrm{O} & -0.594031 & -2.026240 & 0.080212 \\
\mathrm{O} & -1.831490 & -0.000201 & -1.567068 \\
\mathrm{O} & 1.154110 & 0.000687 & 1.104170 \\
\mathrm{O} & 1.180255 & 0.000876 & -1.072203 \\
\mathrm{H} & -0.959388 & 2.607496 & -0.618062 \\
\mathrm{H} & -0.233265 & 2.596852 & 0.786077 \\
\mathrm{H} & -0.238393 & -2.594982 & 0.791702 \\
\mathrm{H} & -0.948064 & -2.609544 & -0.620942 \\
\mathrm{H} & -2.808964 & -0.002567 & -1.515723 \\
\mathrm{H} & -1.585238 & 0.001430 & -2.514708 \\
\mathrm{C} & 1.829709 & 0.000705 & 0.021765 \\
\mathrm{C} & 3.361712 & 0.000541 & 0.074694 \\
\mathrm{H} & 3.822468 & 0.844664 & 0.635698 \\
\mathrm{H} & 3.822115 & -0.842396 & 0.637779 \\
\mathrm{H} & 3.843222 & -0.518408 & -0.880578 \\
\mathrm{H} & 3.843143 & 0.517023 & -0.881959
\end{array}
$$

$\left[\left(\mathrm{CH}_{3} \mathrm{COO}^{-}\right){ }^{4} \mathrm{Cr}\left(\mathrm{H}_{2} \mathrm{O}\right)_{3}\left(\mathrm{OH}^{-}\right)\right]^{+}$ $\mathrm{E}=-620.2501715$

$\begin{array}{lccc}\mathrm{C} & 1.891607 & -0.035874 & 0.007736 \\ \mathrm{O} & 1.198089 & 0.037387 & 1.094189 \\ \mathrm{O} & 1.190253 & -0.097250 & -1.076369 \\ \mathrm{C} & 3.382014 & -0.072259 & 0.003561 \\ \mathrm{H} & 3.697631 & -1.120710 & 0.056064 \\ \mathrm{H} & 3.768907 & 0.353275 & -0.923465 \\ \mathrm{H} & 3.776538 & 0.444983 & 0.879207 \\ \mathrm{CR} & -0.459804 & -0.079832 & 0.008833 \\ \mathrm{O} & -0.562611 & 2.063335 & 0.102356 \\ \mathrm{H} & -1.082723 & 2.601458 & -0.515196 \\ \mathrm{O} & -1.837150 & 0.109404 & -1.589147 \\ \mathrm{H} & -2.498500 & -0.604009 & -1.533435 \\ \mathrm{O} & -2.018988 & -0.077743 & 1.401480 \\ \mathrm{H} & -2.095753 & -0.999502 & 1.712534 \\ \mathrm{O} & -0.799180 & -1.877709 & 0.070420 \\ \mathrm{H} & -0.123043 & -2.540511 & -0.129612 \\ \mathrm{H} & 0.144809 & 2.618830 & 0.466161 \\ \mathrm{H} & -1.432597 & 0.027849 & -2.470332\end{array}$




$$
\begin{aligned}
& \begin{array}{llll}
H & -2.124995 & 0.523713 & 2.154865
\end{array} \\
& \mathrm{HNO}_{3} \\
& \mathrm{E}=-280.9699626 \\
& \begin{array}{llll}
\mathrm{N} & 0.000000 & 0.151222 & 0.000000
\end{array} \\
& \begin{array}{llll}
\mathrm{O} & 1.190278 & 0.451528 & 0.000000
\end{array} \\
& \begin{array}{llll}
\mathrm{O} & -0.984044 & 0.858484 & 0.000000
\end{array} \\
& \begin{array}{llll}
\mathrm{O} & -0.282502 & -1.237937 & 0.000000
\end{array} \\
& \mathrm{H} \quad 0.610145 \quad-1.635156 \quad 0.000000 \\
& \mathrm{NO}_{3}{ }^{-} \\
& \mathrm{E}=-280.4452268 \\
& \begin{array}{llll}
\mathrm{N} & 0.000000 & -0.000387 & 0.000000
\end{array} \\
& \begin{array}{llll}
\mathrm{O} & -0.872138 & 0.930855 & 0.000000
\end{array} \\
& \begin{array}{llll}
\mathrm{O} & -0.370039 & -1.221035 & 0.000000
\end{array} \\
& \begin{array}{llll}
\mathrm{O} & 1.242177 & 0.290519 & 0.000000
\end{array} \\
& {\left[\left(\mathrm{HNO}_{3}\right){ }^{4} \mathrm{Cr}\left(\mathrm{H}_{2} \mathrm{O}\right)_{4}\right]^{3+}} \\
& \mathrm{E}=-672.2634363 \\
& \begin{array}{llll}
\text { CR } & -0.547028 & 0.000086 & 0.001150
\end{array} \\
& \begin{array}{llll}
\mathrm{O} & -0.525142 & 2.011413 & -0.050131
\end{array} \\
& \begin{array}{llll}
\mathrm{O} & -1.943326 & -0.000663 & -1.415341
\end{array} \\
& \begin{array}{llll}
\mathrm{H} & -0.116476 & 2.586897 & -0.733074
\end{array} \\
& \mathrm{H} \quad-0.911831 \quad 2.600815 \quad 0.633455 \\
& \begin{array}{llll}
\mathrm{O} & -1.864565 & -0.000138 & 1.511300
\end{array} \\
& \begin{array}{llll}
\mathrm{O} & -0.523808 & -2.011479 & -0.049651
\end{array} \\
& \begin{array}{llll}
\text { O } & 1.297566 & 0.000343 & 1.087870
\end{array} \\
& \begin{array}{llll}
\mathrm{O} & 1.242514 & 0.000151 & -1.066705
\end{array} \\
& \mathrm{H} \quad-2.313727 \quad 0.791062 \quad-1.862748 \\
& \mathrm{H} \quad-2.313439 \quad-0.792712 \quad-1.862413 \\
& \text { H } \quad-1.664319 \quad-0.000058 \quad 2.473660 \\
& \mathrm{H} \quad-2.842295 \quad-0.000395 \quad 1.415629 \\
& \mathrm{H} \quad-0.909790 \quad-2.601279 \quad 0.633984 \\
& \mathrm{H} \quad-0.115294 \quad-2.586521 \quad-0.733039 \\
& \mathrm{~N} \quad \begin{array}{llll}
1.927066 & 0.000233 & -0.003892
\end{array} \\
& \begin{array}{llll}
\mathrm{O} & 3.214348 & 0.000148 & -0.112100
\end{array} \\
& \begin{array}{llll}
\mathrm{H} & 3.645682 & 0.000301 & 0.792243
\end{array} \\
& {\left[\left(\mathrm{HNO}_{3}\right){ }^{4} \mathrm{Cr}\left(\mathrm{H}_{2} \mathrm{O}\right)_{3}\left(\mathrm{OH}^{-}\right)\right]^{2+}} \\
& \mathrm{E}=-672.2204913 \\
& \begin{array}{lrrc}
\mathrm{N} & 1.941680 & -0.030051 & 0.109965 \\
\mathrm{O} & 3.261329 & -0.007229 & 0.126813 \\
\mathrm{O} & 1.308683 & -0.093088 & -0.980292 \\
\mathrm{H} & 3.577755 & -0.034418 & -0.811602 \\
\mathrm{O} & 1.344691 & 0.016100 & 1.188221 \\
\mathrm{O} & -0.591278 & -2.040463 & -0.063981
\end{array}
\end{aligned}
$$




$\begin{array}{llll}\mathrm{H} & -1.209521 & -2.582070 & 0.461838 \\ \mathrm{H} & -0.253322 & -2.600117 & -0.787768 \\ \mathrm{CR} & -0.631582 & -0.004796 & -0.054408 \\ \mathrm{O} & -2.190335 & -0.094011 & 1.241539 \\ \mathrm{H} & -3.072602 & 0.145259 & 0.896411 \\ \mathrm{H} & -2.202767 & 0.001552 & 2.212092 \\ \mathrm{O} & -0.442801 & 2.024635 & 0.010957 \\ \mathrm{H} & -0.310140 & 2.642476 & 0.752218 \\ \mathrm{H} & -0.894962 & 2.496484 & -0.716516 \\ \mathrm{O} & -1.699323 & 0.275499 & -1.446349 \\ \mathrm{H} & -1.995971 & -0.395238 & -2.085908\end{array}$

$\mathrm{H}_{2} \mathrm{SO}_{4}$

$\mathrm{E}=-700.318481$

$\begin{array}{cccc}\mathrm{S} & 0.011822 & 0.145400 & 0.076799 \\ \mathrm{O} & 1.248820 & -0.814909 & -0.320828 \\ \mathrm{O} & -1.146032 & -0.635039 & -0.782773 \\ \mathrm{H} & 1.189713 & -1.636606 & 0.199777 \\ \mathrm{O} & 0.288136 & 1.430973 & -0.520692 \\ \mathrm{O} & -0.313016 & -0.010128 & 1.489896 \\ \mathrm{H} & -2.002134 & -0.456969 & -0.353380\end{array}$

$\mathrm{HSO}_{4}^{-}$

$\mathrm{E}=-699.8177453$

$\begin{array}{llll}\mathrm{S} & -0.154735 & 0.026868 & 0.000101\end{array}$

$\begin{array}{llll}\mathrm{O} & -0.881339 & -1.260433 & 0.002149\end{array}$

$\begin{array}{llll}\text { O } & -0.269279 & 0.831415 & -1.247046\end{array}$

$\begin{array}{lllll}\text { O } & 1.477766 & -0.499147 & -0.001324\end{array}$

$\begin{array}{llll}\mathrm{O} & -0.266303 & 0.833720 & 1.246065\end{array}$

$\mathrm{H} \quad 1.988993 \quad 0.325685 \quad-0.000363$

$\left[\left(\mathrm{H}_{2} \mathrm{SO}_{4}\right){ }^{4} \mathrm{Cr}\left(\mathrm{H}_{2} \mathrm{O}\right)_{4}\right]^{3+}$

$\mathrm{E}=-1091.6513654$

$\begin{array}{llll}\text { CR } & 0.925345 & 0.010708 & -0.003834\end{array}$

$\begin{array}{llll}\text { O } & 2.404053 & -0.145850 & -1.358752\end{array}$

$\begin{array}{llll}\mathrm{O} & 0.844923 & -2.011311 & -0.003639\end{array}$

$\mathrm{H} \quad 2.836780 \quad 0.587243 \quad-1.843269$

H $\quad 2.716539 \quad-0.982838 \quad-1.761141$

$\begin{array}{llll}\mathrm{O} & 1.086048 & 2.024544 & -0.154542\end{array}$

$\begin{array}{llll}\mathrm{O} & 2.211508 & -0.007935 & 1.547559\end{array}$

$\begin{array}{llll}\mathrm{O} & -0.767257 & 0.066146 & -1.167781\end{array}$

$\begin{array}{llll}\mathrm{O} & -0.777444 & 0.145900 & 1.167709\end{array}$

$\mathrm{H} \quad 1.153428 \quad-2.590561 \quad 0.724194$

$\mathrm{H} \quad \begin{array}{llll}0.433188 & -2.584444 & -0.683781\end{array}$

$\mathrm{H} \quad 0.853582 \quad 2.571780 \quad-0.932869$

$\mathrm{H} \quad 1.524546 \quad 2.607726 \quad 0.499664$ 


\begin{tabular}{|c|c|c|c|}
\hline $\mathrm{H}$ & 3.187375 & -0.083191 & 1.491654 \\
\hline $\mathrm{H}$ & 1.969571 & 0.031079 & 2.497446 \\
\hline $\mathrm{S}$ & -1.731888 & 0.037610 & -0.000554 \\
\hline $\mathrm{O}$ & -2.507836 & -1.288510 & -0.065233 \\
\hline $\mathrm{O}$ & -2.872337 & 1.075162 & -0.011117 \\
\hline $\mathrm{H}$ & -3.460723 & -1.289883 & 0.216788 \\
\hline $\mathrm{H}$ & -2.685629 & 2.009173 & 0.258548 \\
\hline \multicolumn{4}{|c|}{$\begin{array}{l}{\left[\left(\mathrm{H}_{2} \mathrm{SO}_{4}\right){ }^{4} \mathrm{Cr}\left(\mathrm{H}_{2} \mathrm{O}\right)_{3}\left(\mathrm{OH}^{-}\right)\right]^{2+}} \\
\mathrm{E}=-1091.5910736\end{array}$} \\
\hline $\mathrm{CR}$ & -0.914448 & -0.048221 & 0.004714 \\
\hline $\mathrm{S}$ & 1.688301 & 0.095428 & 0.007084 \\
\hline $\mathrm{O}$ & -2.337509 & -0.422733 & -1.376124 \\
\hline $\mathrm{O}$ & 0.801162 & 0.351119 & 1.183677 \\
\hline $\mathrm{O}$ & 2.903113 & 1.048055 & 0.052244 \\
\hline $\mathrm{O}$ & 2.351535 & -1.315918 & -0.001939 \\
\hline $\mathrm{O}$ & 0.789610 & 0.290234 & -1.182555 \\
\hline $\mathrm{O}$ & -1.384586 & 1.994143 & -0.191444 \\
\hline $\mathrm{O}$ & -2.269726 & -0.129827 & 1.520736 \\
\hline $\mathrm{O}$ & -0.415562 & -1.816527 & -0.023241 \\
\hline $\mathrm{H}$ & -2.800520 & 0.237030 & -1.924373 \\
\hline $\mathrm{H}$ & -2.283624 & -1.261686 & -1.872713 \\
\hline $\mathrm{H}$ & -1.862988 & 2.518398 & 0.476018 \\
\hline $\mathrm{H}$ & -0.913476 & 2.622857 & -0.767871 \\
\hline $\mathrm{H}$ & -2.023408 & -0.224087 & 2.460367 \\
\hline $\mathrm{H}$ & -3.187124 & -0.444414 & 1.418127 \\
\hline $\mathrm{H}$ & -0.870027 & -2.491266 & 0.508548 \\
\hline $\mathrm{H}$ & 3.749386 & 0.679658 & -0.296404 \\
\hline $\mathrm{H}$ & 1.621427 & -1.994413 & -0.078989 \\
\hline
\end{tabular}

\begin{tabular}{|c|c|c|c|}
\hline \multicolumn{4}{|c|}{$\begin{array}{l}{\left[\left(\mathrm{HSO}_{4}^{-}\right)^{4} \mathrm{Cr}\left(\mathrm{H}_{2} \mathrm{O}\right)_{4}\right]^{2+}} \\
\mathrm{E}=-10916254266\end{array}$} \\
\hline $\mathrm{CR}$ & -0.835535 & -0.000057 & -0.002900 \\
\hline $\mathrm{S}$ & 1.766897 & -0.126634 & 0.006278 \\
\hline $\mathrm{O}$ & -0.998382 & -2.036048 & -0.119088 \\
\hline $\mathrm{O}$ & 0.723554 & -0.052932 & 1.160197 \\
\hline $\mathrm{O}$ & 2.465804 & 1.295736 & -0.005282 \\
\hline $\mathrm{O}$ & 2.698993 & -1.214435 & 0.005252 \\
\hline $\mathrm{O}$ & 0.724183 & -0.060759 & -1.159782 \\
\hline $\mathrm{O}$ & -2.387553 & 0.047517 & -1.354499 \\
\hline $\mathrm{O}$ & -0.857780 & 2.038607 & -0.131330 \\
\hline $\mathrm{O}$ & -2.162323 & 0.057493 & 1.573803 \\
\hline $\mathrm{H}$ & -1.226408 & -2.604799 & 0.640217 \\
\hline $\mathrm{H}$ & -0.404642 & -2.551284 & -0.699415 \\
\hline $\mathrm{H}$ & -2.637693 & 0.839044 & -1.866050 \\
\hline $\mathrm{H}$ & -2.713537 & -0.733243 & -1.839139 \\
\hline
\end{tabular}




$\begin{array}{rrrr}\mathrm{H} & -1.111352 & 2.623179 & 0.607218 \\ \mathrm{H} & -0.189205 & 2.512332 & -0.663051 \\ \mathrm{H} & -1.897327 & 0.046818 & 2.512948 \\ \mathrm{H} & -3.136142 & 0.069234 & 1.531366 \\ \mathrm{H} & 3.446815 & 1.224812 & -0.009100\end{array}$

$\mathrm{CF}_{3} \mathrm{SO}_{3} \mathrm{H}$

$\mathrm{E}=-962.2035027$

$\begin{array}{lrrc}\mathrm{C} & -1.017795 & 0.005005 & -0.001686 \\ \mathrm{~F} & -1.440747 & -0.197762 & -1.252396 \\ \mathrm{~F} & -1.549122 & -0.911951 & 0.814351 \\ \mathrm{~F} & -1.379312 & 1.234105 & 0.404034 \\ \mathrm{~S} & 0.869444 & -0.145365 & 0.078792 \\ \mathrm{O} & 1.270430 & 0.202449 & 1.434743 \\ \mathrm{O} & 1.233752 & -1.375938 & -0.589693 \\ \mathrm{O} & 1.254475 & 1.086483 & -0.916489 \\ \mathrm{H} & 1.449043 & 1.872330 & -0.372944\end{array}$

$\mathrm{CF}_{3} \mathrm{SO}_{3}^{-}$

$\mathrm{E}=-961.7168269$

$\begin{array}{cccc}\text { C } & 0.956198 & 0.000055 & -0.000038 \\ \text { F } & 1.463169 & 1.263117 & 0.026344 \\ \text { F } & 1.462882 & -0.654388 & 1.080654 \\ \text { F } & 1.463189 & -0.608719 & -1.106964 \\ \text { S } & -0.942666 & -0.000001 & -0.000032 \\ \text { O } & -1.256428 & -1.448800 & -0.028998 \\ \text { O } & -1.256500 & 0.699274 & 1.269165 \\ \text { O } & -1.256785 & 0.749477 & -1.240112\end{array}$

$\left[\left(\mathrm{CF}_{3} \mathrm{SO}_{3} \mathrm{H}\right){ }^{4} \mathrm{Cr}\left(\mathrm{H}_{2} \mathrm{O}\right)_{4}\right]^{3+}$

$\mathrm{E}=-1353.5285439$

$\begin{array}{lllc}\mathrm{CR} & -1.520158 & -0.081126 & -0.010755 \\ \mathrm{O} & -2.754996 & -0.354243 & 1.555321 \\ \mathrm{O} & -2.973803 & -0.442238 & -1.352302 \\ \mathrm{H} & -2.506215 & -0.276004 & 2.501091 \\ \mathrm{H} & -3.708599 & -0.577208 & 1.513010 \\ \mathrm{O} & -1.111194 & -2.061023 & -0.056928 \\ \mathrm{O} & -2.045604 & 1.869080 & -0.105002 \\ \mathrm{O} & 0.125182 & 0.340804 & 1.157787 \\ \mathrm{O} & 0.138425 & 0.302170 & -1.197866 \\ \mathrm{H} & -3.165109 & -1.301198 & -1.782345 \\ \mathrm{H} & -3.534133 & 0.233995 & -1.786294 \\ \mathrm{H} & -1.323801 & -2.699106 & 0.655571 \\ \mathrm{H} & -0.642415 & -2.549675 & -0.764199 \\ \mathrm{H} & -2.488967 & 2.366953 & 0.613213 \\ \mathrm{H} & -1.816809 & 2.508370 & -0.810812\end{array}$




$\begin{array}{lrrr}\text { S } & 1.051649 & 0.597452 & -0.013032 \\ \text { O } & 1.505435 & 2.081685 & 0.073328 \\ \text { H } & 2.316431 & 2.371228 & -0.420740 \\ \text { C } & 2.604133 & -0.488895 & 0.007036 \\ \text { F } & 3.132611 & -0.325545 & 1.189119 \\ \text { F } & 2.164572 & -1.712001 & -0.204095 \\ \text { F } & 3.351115 & -0.034238 & -0.972668\end{array}$

$\left[\left(\mathrm{CF}_{3} \mathrm{SO}_{3} \mathrm{H}\right){ }^{4} \mathrm{Cr}\left(\mathrm{H}_{2} \mathrm{O}\right)_{3}\left(\mathrm{OH}^{-}\right)\right]^{2+}$

$\mathrm{E}=-1353.4673431$

$\begin{array}{lccc}\text { CR } & -1.605405 & -0.023127 & 0.006665 \\ \mathrm{O} & -1.647402 & -2.123973 & -0.161618 \\ \mathrm{O} & -2.956236 & -0.209017 & 1.520162 \\ \mathrm{H} & -1.077651 & -2.651951 & -0.749041 \\ \mathrm{H} & -2.019103 & -2.729983 & 0.504195 \\ \mathrm{O} & -3.073176 & 0.025211 & -1.380216 \\ \mathrm{O} & -1.503425 & 1.809862 & -0.048894 \\ \mathrm{H} & -3.917787 & -0.087237 & 1.412280 \\ \mathrm{H} & -2.735826 & -0.042348 & 2.456076 \\ \mathrm{H} & -3.185571 & 0.852602 & -1.886339 \\ \mathrm{H} & -3.385680 & -0.720399 & -1.924409 \\ \mathrm{H} & -2.094144 & 2.374011 & 0.477746 \\ \mathrm{~S} & 0.977940 & 0.394593 & 0.003121 \\ \mathrm{O} & 1.307360 & 1.922681 & -0.018264 \\ \mathrm{O} & 0.130806 & -0.020421 & -1.184439 \\ \mathrm{O} & 0.144572 & -0.020407 & 1.197174 \\ \mathrm{H} & 0.445818 & 2.426567 & -0.071200 \\ \mathrm{C} & 2.828312 & -0.377005 & 0.005043 \\ \mathrm{~F} & 3.387756 & 0.061331 & 1.100259 \\ \mathrm{~F} & 2.628494 & -1.675573 & 0.005447 \\ \mathrm{~F} & 3.390722 & 0.059892 & -1.089115\end{array}$

$\left[\left(\mathrm{CF}_{3} \mathrm{SO}_{3}^{-}\right)^{4} \mathrm{Cr}\left(\mathrm{H}_{2} \mathrm{O}\right)_{4}\right]^{2+}$

$\mathrm{E}=-1353.4981066$

$\begin{array}{lccc}\mathrm{CR} & -1.413856 & -0.083648 & -0.003303 \\ \mathrm{O} & -0.989096 & -2.074414 & -0.120115 \\ \mathrm{O} & -2.705792 & -0.448138 & 1.562594 \\ \mathrm{H} & -0.183329 & -2.390599 & -0.571901 \\ \mathrm{H} & -1.194817 & -2.710024 & 0.590575 \\ \mathrm{O} & -2.062857 & 1.855315 & -0.117602 \\ \mathrm{O} & -2.903651 & -0.499517 & -1.361747 \\ \mathrm{H} & -3.655746 & -0.661181 & 1.510723 \\ \mathrm{H} & -2.460309 & -0.371562 & 2.504131 \\ \mathrm{H} & -1.624194 & 2.500058 & -0.706901 \\ \mathrm{H} & -2.404936 & 2.352275 & 0.649441 \\ \mathrm{H} & -3.412479 & 0.181067 & -1.840061\end{array}$




$\begin{array}{lccc}\text { H } & -2.936180 & -1.318747 & -1.889805 \\ \text { S } & 1.043454 & 0.798634 & 0.008465 \\ \text { O } & 0.084302 & 0.357640 & 1.168330 \\ \text { O } & 0.083888 & 0.367273 & -1.164903 \\ \text { O } & 1.517822 & 2.155482 & 0.006921 \\ \text { C } & 2.525261 & -0.450746 & -0.000144 \\ \text { F } & 3.144870 & -0.324777 & 1.150674 \\ \text { F } & 1.992115 & -1.677878 & -0.132034 \\ \text { F } & 3.280881 & -0.148072 & -1.028567\end{array}$

Geometries of Met(III)-addition to glycine (Table 4).

\begin{tabular}{crrr}
\multicolumn{5}{c}{$\left[{ }^{4} \mathrm{Cr}\left(\mathrm{H}_{2} \mathrm{O}\right)_{6}\right]^{3+}$} \\
$\mathrm{E}=-544.2909777$ \\
$\mathrm{O}$ & 0.000000 & 0.000000 & 2.019812 \\
$\mathrm{H}$ & 0.791318 & 0.000000 & 2.599427 \\
$\mathrm{H}$ & -0.791318 & 0.000000 & 2.599427 \\
$\mathrm{O}$ & 0.000000 & 2.019812 & 0.000000 \\
$\mathrm{H}$ & 0.000000 & 2.599427 & 0.791318 \\
$\mathrm{H}$ & 0.000000 & 2.599427 & -0.791318 \\
$\mathrm{O}$ & 2.019812 & 0.000000 & 0.000000 \\
$\mathrm{H}$ & 2.599427 & 0.791318 & 0.000000 \\
$\mathrm{H}$ & 2.599427 & -0.791318 & 0.000000 \\
$\mathrm{O}$ & 0.000000 & 0.000000 & -2.019812 \\
$\mathrm{H}$ & -0.791318 & 0.000000 & -2.599427 \\
$\mathrm{H}$ & 0.791318 & 0.000000 & -2.599427 \\
$\mathrm{O}$ & -2.019812 & 0.000000 & 0.000000 \\
$\mathrm{H}$ & -2.599427 & -0.791318 & 0.000000 \\
$\mathrm{H}$ & -2.599427 & 0.791318 & 0.000000 \\
$\mathrm{O}$ & 0.000000 & -2.019812 & 0.000000 \\
$\mathrm{H}$ & 0.000000 & -2.599427 & 0.791318 \\
$\mathrm{H}$ & 0.000000 & -2.599427 & -0.791318 \\
$\mathrm{CR}$ & 0.000000 & 0.000000 & 0.000000
\end{tabular}

$\left[(\text { Glycine }){ }^{4} \mathrm{Cr}\left(\mathrm{H}_{2} \mathrm{O}\right)_{3}\left(\mathrm{OH}^{-}\right)\right]^{+2}$ (Equation 5) $\mathrm{E}=-675.8636629$
$\begin{array}{llll}\text { C } & 1.474613 & -0.058266 & 0.417153\end{array}$
$\begin{array}{llll}\text { C } & 2.973108 & -0.110926 & 0.673295\end{array}$
$\begin{array}{lllll}\mathrm{O} & 1.036836 & 0.001412 & -0.785395\end{array}$
$\begin{array}{lllll}\mathrm{N} & 3.727332 & 0.106446 & -0.620264\end{array}$
$\begin{array}{llll}\mathrm{H} & 3.271786 & 0.650329 & 1.396217\end{array}$
$\begin{array}{llll}\mathrm{H} & 3.242119 & -1.092709 & 1.070295\end{array}$
$\begin{array}{llll}\mathrm{O} & 0.651605 & -0.092723 & 1.378144\end{array}$
$\mathrm{H} \quad 3.691551 \quad 1.088158 \quad-0.919909$
H $\quad 4.716178 \quad-0.155936 \quad-0.524317$ 


$\begin{array}{cccc}\mathrm{H} & 3.308761 & -0.448849 & -1.378785 \\ \mathrm{O} & -2.630271 & 0.037127 & 1.042108 \\ \mathrm{H} & -3.457123 & 0.040131 & 0.525858 \\ \mathrm{H} & -2.830014 & -0.162298 & 1.971523 \\ \mathrm{O} & -0.907266 & -2.051083 & -0.161309 \\ \mathrm{H} & -1.520788 & -2.416191 & -0.824544 \\ \mathrm{H} & -0.864080 & -2.666790 & 0.589682 \\ \mathrm{O} & -0.811273 & 2.054056 & 0.017496 \\ \mathrm{H} & -1.386257 & 2.479390 & -0.645306 \\ \mathrm{H} & -0.894945 & 2.554190 & 0.847759 \\ \mathrm{CR} & -0.884774 & 0.001176 & -0.070659 \\ \mathrm{O} & -1.981522 & 0.084822 & -1.513141 \\ \mathrm{H} & -1.685143 & 0.103472 & -2.436718\end{array}$

$\left[(\text { Glycine }){ }^{4} \mathrm{Cr}\left(\mathrm{H}_{2} \mathrm{O}\right)_{4}\right]^{+3}$ (Equation 4)

$\mathrm{E}=-675.9684581$

$\begin{array}{cccc}\mathrm{C} & 2.977106 & -0.004197 & -0.635472 \\ \mathrm{H} & 3.213284 & 0.874215 & -1.245114 \\ \mathrm{H} & 3.213284 & -0.890336 & -1.233877 \\ \mathrm{C} & 1.490793 & -0.002290 & -0.322982 \\ \mathrm{O} & 0.636426 & -0.004606 & -1.275857 \\ \mathrm{O} & 0.995958 & 0.001445 & 0.864945 \\ \mathrm{CR} & -0.846606 & 0.000111 & 0.026448 \\ \mathrm{~N} & 3.860770 & 0.003573 & 0.590022 \\ \mathrm{H} & 3.724356 & -0.822666 & 1.188364 \\ \mathrm{H} & 4.851807 & 0.002364 & 0.300320 \\ \mathrm{O} & -0.930714 & 2.030532 & 0.100569 \\ \mathrm{O} & -0.933634 & -2.029699 & 0.111875 \\ \mathrm{O} & -2.195602 & 0.005559 & 1.570851 \\ \mathrm{H} & -2.537602 & 0.793582 & 2.036332 \\ \mathrm{O} & -2.330747 & -0.002900 & -1.376279 \\ \mathrm{H} & -3.295371 & -0.001632 & -1.216045 \\ \mathrm{H} & -2.187983 & -0.005763 & -2.344396 \\ \mathrm{H} & -0.471567 & 2.616727 & 0.732256 \\ \mathrm{H} & -1.332016 & 2.593477 & -0.590829 \\ \mathrm{H} & -2.538028 & -0.779248 & 2.041434 \\ \mathrm{H} & -1.336683 & -2.595723 & -0.575991 \\ \mathrm{H} & -0.474733 & -2.613223 & 0.746210 \\ \mathrm{H} & 3.723524 & 0.836841 & 1.178333\end{array}$

$\left[{ }^{6} \mathrm{Fe}\left(\mathrm{H}_{2} \mathrm{O}\right)_{6}\right]^{3+}$

$\mathrm{E}=--581.0284859$
$\begin{array}{llll}\text { FE } & -0.000001 & 0.000012 & 0.000000\end{array}$
$\begin{array}{llll}\mathrm{O} & -2.097364 & 0.006281 & 0.000450\end{array}$
$\begin{array}{llll}\mathrm{O} & 0.006685 & 2.015885 & 0.004502\end{array}$
$\begin{array}{llll}\text { O } & 2.097369 & -0.006367 & -0.000465\end{array}$ 


$\begin{array}{cccc}\mathrm{O} & -0.006651 & -2.015867 & -0.004491 \\ \mathrm{O} & -0.000491 & 0.004887 & -1.917370 \\ \mathrm{O} & 0.000442 & -0.004854 & 1.917369 \\ \mathrm{H} & -0.003284 & -0.796257 & -2.486114 \\ \mathrm{H} & 0.001382 & 0.809205 & -2.481619 \\ \mathrm{H} & 0.805155 & 2.584324 & 0.004618 \\ \mathrm{H} & -0.787792 & 2.589880 & 0.008776 \\ \mathrm{H} & -2.685236 & 0.010877 & -0.783695 \\ \mathrm{H} & -2.685021 & 0.006536 & 0.784773 \\ \mathrm{H} & 0.787845 & -2.589844 & -0.006798 \\ \mathrm{H} & -0.805108 & -2.584320 & -0.006514 \\ \mathrm{H} & 2.685014 & -0.005943 & -0.784797 \\ \mathrm{H} & 2.685255 & -0.011618 & 0.783664 \\ \mathrm{H} & 0.005069 & 0.796288 & 2.486105 \\ \mathrm{H} & -0.003177 & -0.809161 & 2.481627\end{array}$

$\left[(\text { Glycine }){ }^{6} \mathrm{Fe}\left(\mathrm{H}_{2} \mathrm{O}\right)_{3}\left(\mathrm{OH}^{-}\right)\right]^{+2}$ (Equation 5) $\mathrm{E}=-712.6582755$

$\begin{array}{cccc}\mathrm{O} & -0.684909 & -1.168576 & -0.579529 \\ \mathrm{~N} & -3.840231 & 0.524763 & 0.141654 \\ \mathrm{H} & -3.280270 & -1.508205 & 0.452518 \\ \mathrm{H} & -3.270325 & -1.004914 & -1.248737 \\ \mathrm{O} & -1.183026 & 0.704627 & 0.483791 \\ \mathrm{H} & -4.753125 & 0.275022 & 0.538705 \\ \mathrm{O} & 1.122101 & -1.132115 & 1.628084 \\ \mathrm{H} & 1.571096 & -0.844004 & 2.440214 \\ \mathrm{H} & 0.714744 & -1.997311 & 1.797286 \\ \mathrm{O} & 2.583990 & -0.998648 & -0.752280 \\ \mathrm{H} & 2.969745 & -1.792184 & -0.345382 \\ \mathrm{H} & 2.920655 & -0.918333 & -1.661807 \\ \mathrm{O} & 1.737362 & 1.504738 & 1.210803 \\ \mathrm{H} & 2.519356 & 2.023452 & 0.950977 \\ \mathrm{H} & 1.140223 & 2.114376 & 1.678515 \\ \mathrm{H} & -3.304597 & 1.079490 & 0.827835 \\ \mathrm{O} & 0.997038 & 1.076748 & -1.628806 \\ \mathrm{H} & 1.070744 & 1.840815 & -2.217802 \\ \mathrm{FE} & 0.901095 & 0.091488 & -0.170543 \\ \mathrm{H} & -4.008280 & 1.130666 & -0.669612 \\ \mathrm{C} & -1.543506 & -0.361582 & -0.100897 \\ \mathrm{C} & -3.026041 & -0.695930 & -0.231880\end{array}$

$\left[(\text { Glycine }){ }^{6} \mathrm{Fe}\left(\mathrm{H}_{2} \mathrm{O}\right)_{4}\right]^{+3}$ (Equation 4) $\mathrm{E}=-712.7527845$
$\begin{array}{llll}\text { C } & 3.029655 & 0.003142 & -0.604216\end{array}$
$\begin{array}{llll}\mathrm{H} & 3.274288 & 0.890386 & -1.197213\end{array}$
H $\quad 3.277597 \quad-0.874476-1.209916$ 


$\begin{array}{lccc}\mathrm{C} & 1.537355 & -0.000873 & -0.317088 \\ \mathrm{O} & 0.706834 & 0.000864 & -1.283309 \\ \mathrm{O} & 1.035541 & -0.004599 & 0.869390 \\ \mathrm{FE} & -0.865146 & -0.001050 & 0.052012 \\ \mathrm{~N} & 3.895211 & -0.003826 & 0.634733 \\ \mathrm{H} & 3.751035 & -0.838970 & 1.218510 \\ \mathrm{H} & 4.890571 & 0.002142 & 0.361032 \\ \mathrm{O} & -0.979365 & 2.071582 & 0.203573 \\ \mathrm{O} & -0.990198 & -2.072617 & 0.197213 \\ \mathrm{O} & -2.336196 & 0.002401 & 1.501367 \\ \mathrm{H} & -2.743772 & 0.790496 & 1.912468 \\ \mathrm{O} & -2.060995 & 0.005139 & -1.623877 \\ \mathrm{H} & -3.037754 & 0.009287 & -1.682315 \\ \mathrm{H} & -1.707083 & 0.004668 & -2.536832 \\ \mathrm{H} & -0.585819 & 2.637298 & 0.896183 \\ \mathrm{H} & -1.341184 & 2.663660 & -0.485560 \\ \mathrm{H} & -2.746063 & -0.783221 & 1.914914 \\ \mathrm{H} & -1.361105 & -2.660142 & -0.491036 \\ \mathrm{H} & -0.595133 & -2.643194 & 0.884990 \\ \mathrm{H} & 3.744695 & 0.820371 & 1.232299\end{array}$

$\begin{array}{cccc}{\left[{ }^{1} \mathrm{Al}\left(\mathrm{H}_{2} \mathrm{O}\right)_{6}\right]^{3+}} & & \\ \mathrm{E}=-700.2534589 & & \\ \mathrm{AL} & 0.000000 & 0.000000 & 0.000000 \\ \mathrm{O} & 0.000000 & 0.000000 & 1.941523 \\ \mathrm{O} & 0.000000 & 0.000000 & -1.941523 \\ \mathrm{H} & 0.000000 & -0.788072 & 2.524751 \\ \mathrm{H} & 0.000000 & 0.788072 & 2.524751 \\ \mathrm{O} & 0.000000 & 1.941523 & 0.000000 \\ \mathrm{O} & 0.000000 & -1.941523 & 0.000000 \\ \mathrm{O} & 1.941523 & 0.000000 & 0.000000 \\ \mathrm{O} & -1.941523 & 0.000000 & 0.000000 \\ \mathrm{H} & 0.000000 & 0.788072 & -2.524751 \\ \mathrm{H} & 0.000000 & -0.788072 & -2.524751 \\ \mathrm{H} & 0.788072 & 2.524751 & 0.000000 \\ \mathrm{H} & -0.788072 & 2.524751 & 0.000000 \\ \mathrm{H} & -0.788072 & -2.524751 & 0.000000 \\ \mathrm{H} & 0.788072 & -2.524751 & 0.000000 \\ \mathrm{H} & 2.524751 & 0.000000 & -0.788072 \\ \mathrm{H} & 2.524751 & 0.000000 & 0.788072 \\ \mathrm{H} & -2.524751 & 0.000000 & 0.788072 \\ \mathrm{H} & -2.524751 & 0.000000 & -0.788072\end{array}$

$\left[(\text { Glycine })^{1} \mathrm{Al}\left(\mathrm{H}_{2} \mathrm{O}\right)_{3}\left(\mathrm{OH}^{-}\right)\right]^{+2}$ (Equation 5) $\mathrm{E}=-831.8247049$

$\begin{array}{llll}\text { C } & 2.756461 & 0.000028 & -0.717404\end{array}$ 


$\begin{array}{cccc}\mathrm{H} & 2.996143 & 0.886335 & -1.309105 \\ \mathrm{H} & 2.995484 & -0.886685 & -1.308780 \\ \mathrm{C} & 1.289266 & 0.000474 & -0.327340 \\ \mathrm{O} & 0.378110 & 0.000729 & -1.227456 \\ \mathrm{O} & 0.936117 & 0.000397 & 0.886134 \\ \mathrm{AL} & -1.013391 & 0.000056 & 0.143893 \\ \mathrm{~N} & 3.613876 & -0.000125 & 0.528050 \\ \mathrm{H} & 3.421973 & -0.823188 & 1.113054 \\ \mathrm{H} & 4.613718 & -0.000216 & 0.289725 \\ \mathrm{O} & -0.946168 & 1.997499 & 0.110957 \\ \mathrm{O} & -0.944125 & -1.997409 & 0.111354 \\ \mathrm{O} & -2.125187 & -0.000005 & 1.451457 \\ \mathrm{O} & -2.443629 & -0.001857 & -1.187278 \\ \mathrm{H} & -3.359813 & -0.001385 & -0.855669 \\ \mathrm{H} & -2.439085 & 0.002630 & -2.158250 \\ \mathrm{H} & -1.538897 & 2.392471 & 0.776210 \\ \mathrm{H} & -0.894365 & 2.596122 & -0.652500 \\ \mathrm{H} & -2.023590 & 0.000541 & 2.407990 \\ \mathrm{H} & -0.896614 & -2.594766 & -0.653400 \\ \mathrm{H} & -1.535490 & -2.392500 & 0.777758 \\ \mathrm{H} & 3.422170 & 0.822946 & 1.113125\end{array}$

$\left[(\text { Glycine })^{1} \mathrm{Al}\left(\mathrm{H}_{2} \mathrm{O}\right)_{4}\right]^{+3}$ (Equation 4) $\mathrm{E}=-831.9250302$

$\begin{array}{lrrc}\mathrm{C} & -2.812072 & -0.000068 & 0.641655 \\ \mathrm{H} & -3.047395 & 0.881600 & 1.246285 \\ \mathrm{H} & -3.047403 & -0.881866 & 1.246094 \\ \mathrm{C} & -1.327316 & -0.000051 & 0.325086 \\ \mathrm{O} & -0.472210 & -0.000058 & 1.273087 \\ \mathrm{O} & -0.837205 & -0.000021 & -0.866383 \\ \mathrm{AL} & 0.945071 & -0.000001 & -0.032655 \\ \mathrm{~N} & -3.700843 & 0.000064 & -0.580435 \\ \mathrm{H} & -3.563560 & -0.828990 & -1.174168 \\ \mathrm{H} & -4.691192 & -0.000059 & -0.289310 \\ \mathrm{O} & 1.058291 & 1.960894 & -0.148445 \\ \mathrm{O} & 1.058366 & -1.960874 & -0.148612 \\ \mathrm{O} & 2.171396 & 0.000102 & -1.508667 \\ \mathrm{H} & 2.503721 & 0.790362 & -1.978677 \\ \mathrm{O} & 2.273719 & -0.000037 & 1.358520 \\ \mathrm{H} & 3.248310 & 0.000012 & 1.274156 \\ \mathrm{H} & 2.049595 & -0.000097 & 2.312301 \\ \mathrm{H} & 0.550929 & 2.531910 & -0.756984 \\ \mathrm{H} & 1.471636 & 2.541672 & 0.520770 \\ \mathrm{H} & 2.503692 & -0.790094 & -1.978806 \\ \mathrm{H} & 1.471848 & -2.541690 & 0.520487 \\ \mathrm{H} & 0.550957 & -2.531865 & -0.757137\end{array}$




$$
\begin{aligned}
& \text { H } \quad-3.563692 \quad 0.829336 \quad-1.173893 \\
& {\left[{ }^{1} \mathrm{Sc}\left(\mathrm{H}_{2} \mathrm{O}\right)_{6}\right]^{3+}} \\
& \mathrm{E}=-1218.5071573 \\
& \begin{array}{llll}
\mathrm{SC} & 0.000006 & 0.000000 & 0.000006
\end{array} \\
& \begin{array}{llll}
\mathrm{O} & 0.633684 & 1.850431 & -0.941159
\end{array} \\
& \begin{array}{llll}
\mathrm{O} & 1.753366 & 0.050368 & 1.278392
\end{array} \\
& \begin{array}{llll}
\text { O } & -0.633696 & -1.850442 & 0.941132
\end{array} \\
& \begin{array}{lllll}
\mathrm{O} & -1.753363 & -0.050365 & -1.278373
\end{array} \\
& \begin{array}{llll}
\text { O } & 1.112095 & -1.133796 & -1.479249
\end{array} \\
& \begin{array}{llll}
\mathrm{O} & -1.112098 & 1.133804 & 1.479244
\end{array} \\
& \text { H } \quad 0.786539 \quad-1.463560 \quad-2.344038 \\
& \mathrm{H} \quad 2.047358 \quad-1.425145 \quad-1.425067 \\
& \begin{array}{llll}
\mathrm{H} & 2.006404 & -0.600961 & 1.967109
\end{array} \\
& \begin{array}{llll}
\mathrm{H} & 2.461505 & 0.729341 & 1.290013
\end{array} \\
& \mathrm{H} \quad 1.206786 \quad 1.950200 \quad-1.731222 \\
& \mathrm{H} \quad 0.407320 \quad 2.764821 \quad-0.666899 \\
& \mathrm{H} \quad-2.461707 \quad-0.729129 \quad-1.289717 \\
& \mathrm{H} \quad-2.006206 \quad 0.600763 \quad-1.967351 \\
& \mathrm{H} \quad-0.407134 \quad-2.764819 \quad 0.666996 \\
& \mathrm{H} \quad-1.206966-1.950235 \quad 1.731071 \\
& \mathrm{H} \quad-0.786762 \quad 1.462988 \quad 2.344336 \\
& \text { H } \quad-2.047163 \quad 1.425726 \quad 1.424747
\end{aligned}
$$

$\left[(\text { Glycine })^{1} \mathrm{Sc}\left(\mathrm{H}_{2} \mathrm{O}\right)_{3}\left(\mathrm{OH}^{-}\right)\right]^{+2}$ (Equation 5)

$\mathrm{E}=-1350.0708252$

$\begin{array}{lrrr}\mathrm{SC} & 0.938274 & 0.129765 & 0.180320 \\ \mathrm{O} & 2.234939 & -1.632776 & 0.592517 \\ \mathrm{O} & 1.197657 & -0.670866 & -1.973255 \\ \mathrm{H} & 1.858925 & -2.526228 & 0.685088 \\ \mathrm{H} & 2.990302 & -1.588448 & 1.205429 \\ \mathrm{O} & 2.284753 & 1.665638 & -0.665705 \\ \mathrm{O} & -1.134812 & 0.727709 & -0.439794 \\ \mathrm{O} & 1.096965 & 0.940129 & 1.817270 \\ \mathrm{O} & -0.763419 & -1.256877 & 0.476536 \\ \mathrm{H} & 1.824450 & -1.367917 & -2.230996 \\ \mathrm{H} & 0.671725 & -0.467792 & -2.764100 \\ \mathrm{H} & 2.664319 & 1.790966 & -1.551103 \\ \mathrm{H} & 2.591008 & 2.400024 & -0.105333 \\ \mathrm{C} & -1.561145 & -0.375865 & 0.053462 \\ \mathrm{H} & 1.173154 & 1.289518 & 2.714378 \\ \mathrm{C} & -3.067891 & -0.616702 & 0.121232 \\ \mathrm{~N} & -3.785514 & 0.689330 & -0.150945 \\ \mathrm{H} & -3.358353 & -1.004335 & 1.097876 \\ \mathrm{H} & -3.353816 & -1.342178 & -0.643437 \\ \mathrm{H} & -3.152386 & 1.293022 & -0.701742\end{array}$




$$
\begin{array}{lrrr}
H & -4.664714 & 0.553601 & -0.661974 \\
H & -4.004226 & 1.191139 & 0.717087
\end{array}
$$

$\left[(\text { Glycine })^{1} \mathrm{Sc}\left(\mathrm{H}_{2} \mathrm{O}\right)_{4}\right]^{+3}$ (Equation 4)

$\mathrm{E}=-1350.1738195$

$\begin{array}{llcc}\mathrm{C} & 3.044370 & -0.000283 & 0.584228 \\ \mathrm{H} & 3.297489 & -0.882925 & 1.179710 \\ \mathrm{H} & 3.297442 & 0.881738 & 1.180669 \\ \mathrm{C} & 1.548474 & -0.000117 & 0.305548 \\ \mathrm{O} & 0.732546 & -0.001153 & 1.285366 \\ \mathrm{O} & 1.058460 & 0.001096 & -0.886057 \\ \mathrm{SC} & -0.921416 & 0.000006 & -0.058817 \\ \mathrm{~N} & 3.892655 & 0.000467 & -0.666116 \\ \mathrm{H} & 3.731502 & 0.829296 & -1.253691 \\ \mathrm{H} & 4.892092 & 0.000530 & -0.410193 \\ \mathrm{O} & -1.074168 & -2.179150 & -0.214029 \\ \mathrm{O} & -1.074810 & 2.179276 & -0.211565 \\ \mathrm{O} & -2.501646 & 0.000771 & -1.575465 \\ \mathrm{H} & -2.935057 & -0.777007 & -1.980688 \\ \mathrm{O} & -2.075200 & -0.001010 & 1.776048 \\ \mathrm{H} & -3.043388 & -0.001584 & 1.921317 \\ \mathrm{H} & -1.662852 & -0.001140 & 2.664817 \\ \mathrm{H} & -0.739271 & -2.760602 & -0.925081 \\ \mathrm{H} & -1.436303 & -2.776642 & 0.471006 \\ \mathrm{H} & -2.933956 & 0.779046 & -1.980917 \\ \mathrm{H} & -1.439424 & 2.775735 & 0.473053 \\ \mathrm{H} & -0.737480 & 2.761783 & -0.920599 \\ \mathrm{H} & 3.731822 & -0.827858 & -1.254484\end{array}$

$\left[{ }^{1} \mathrm{Y}\left(\mathrm{H}_{2} \mathrm{O}\right)_{8}\right]^{3+}$

$\mathrm{E}=-649.1800494$

$\begin{array}{rrrr}\mathrm{O} & 0.000000 & 2.047598 & 1.298952 \\ \mathrm{H} & 0.558982 & 2.836354 & 1.171778 \\ \mathrm{H} & -0.573139 & 2.251675 & 2.061110 \\ \mathrm{O} & -2.047598 & 0.000000 & 1.298952 \\ \mathrm{H} & -2.836354 & 0.558982 & 1.171778 \\ \mathrm{H} & -2.251675 & -0.573139 & 2.061110 \\ \mathrm{O} & 0.000000 & -2.047598 & 1.298952 \\ \mathrm{H} & -0.558982 & -2.836354 & 1.171778 \\ \mathrm{H} & 0.573139 & -2.251675 & 2.061110 \\ \mathrm{O} & 2.047598 & 0.000000 & 1.298952 \\ \mathrm{H} & 2.836354 & -0.558982 & 1.171778 \\ \mathrm{H} & 2.251675 & 0.573139 & 2.061110 \\ \mathrm{O} & 1.447871 & 1.447871 & -1.298952 \\ \mathrm{H} & 2.400865 & 1.610346 & -1.171778 \\ \mathrm{H} & 1.186904 & 1.997445 & -2.061110\end{array}$




$\begin{array}{lrrr}\mathrm{O} & -1.447871 & 1.447871 & -1.298952 \\ \mathrm{H} & -1.610346 & 2.400865 & -1.171778 \\ \mathrm{H} & -1.997445 & 1.186904 & -2.061110 \\ \mathrm{O} & -1.447871 & -1.447871 & -1.298952 \\ \mathrm{H} & -2.400865 & -1.610346 & -1.171778 \\ \mathrm{H} & -1.186904 & -1.997445 & -2.061110 \\ \mathrm{O} & 1.447871 & -1.447871 & -1.298952 \\ \mathrm{H} & 1.610346 & -2.400865 & -1.171778 \\ \mathrm{H} & 1.997445 & -1.186904 & -2.061110 \\ \mathrm{Y} & 0.000000 & 0.000000 & 0.000000\end{array}$

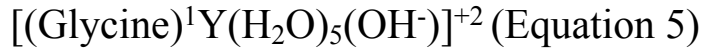
$\mathrm{E}=-780.677546$

$\begin{array}{crrc}\mathrm{Y} & -0.737011 & 0.020649 & 0.139509 \\ \mathrm{O} & -0.907193 & 2.468081 & -0.099388 \\ \mathrm{O} & -1.341681 & -2.080815 & -0.983935 \\ \mathrm{H} & -0.250679 & 3.157178 & -0.282730 \\ \mathrm{H} & -1.345916 & 2.704766 & 0.737910 \\ \mathrm{O} & -1.643120 & 0.678440 & 1.882804 \\ \mathrm{H} & -2.209511 & -2.512156 & -1.024377 \\ \mathrm{H} & -0.679964 & -2.714284 & -1.304202 \\ \mathrm{H} & -1.863524 & 0.902488 & 2.793224 \\ \mathrm{C} & 2.046558 & -0.275995 & -0.277288 \\ \mathrm{C} & 3.566016 & -0.441994 & -0.425894 \\ \mathrm{O} & 1.628758 & 0.756788 & 0.348526 \\ \mathrm{O} & 1.282148 & -1.145689 & -0.765176 \\ \mathrm{~N} & 4.230194 & 0.688289 & 0.335681 \\ \mathrm{H} & 3.849544 & -0.389975 & -1.477614 \\ \mathrm{H} & 3.890707 & -1.401109 & -0.023110 \\ \mathrm{H} & 4.763704 & 0.357936 & 1.145329 \\ \mathrm{H} & 3.443259 & 1.283444 & 0.670866 \\ \mathrm{H} & 4.859770 & 1.246909 & -0.247839 \\ \mathrm{O} & -0.083360 & -1.638292 & 1.796732 \\ \mathrm{H} & 0.201320 & -2.564005 & 1.767889 \\ \mathrm{H} & -0.502802 & -1.480628 & 2.658145 \\ \mathrm{O} & -3.144831 & 0.031550 & -0.304815 \\ \mathrm{H} & -3.782046 & 0.152619 & -1.024945 \\ \mathrm{H} & -3.553232 & 0.349575 & 0.519854 \\ \mathrm{O} & -0.748571 & 0.676311 & -2.237875 \\ \mathrm{H} & -1.096197 & 1.519280 & -2.569190 \\ \mathrm{H} & -0.604982 & 0.101540 & -3.005714\end{array}$

$\left[(\text { Glycine })^{1} \mathrm{Y}\left(\mathrm{H}_{2} \mathrm{O}\right)_{6}\right]^{+3}$ (Equation 4) $\mathrm{E}=-\mathbf{7 8 0 . 8 3 5 0 9 0 3}$

$\begin{array}{llll}\mathrm{Y} & -0.704048 & 0.037822 & -0.029380\end{array}$

$\begin{array}{llll}\mathrm{O} & -3.035378 & 0.457601 & 0.503692\end{array}$ 


\begin{tabular}{|c|c|c|c|}
\hline $\mathrm{O}$ & 0.358682 & 0.926564 & \\
\hline $\mathrm{H}$ & -3.805378 & & -0.042677 \\
\hline $\mathrm{H}$ & -3.401064 & & \\
\hline $\mathrm{O}$ & -0.598048 & 0.367875 & 2.351364 \\
\hline $\mathrm{O}$ & -1.942511 & -1.286986 & -1.650110 \\
\hline $\mathrm{O}$ & -1.209115 & -2.120476 & 071 \\
\hline $\mathrm{O}$ & -0.767 & & \\
\hline $\mathrm{O}$ & 1.15 & -1.3 & \\
\hline $\mathrm{O}$ & 1.66 & & \\
\hline $\mathrm{H}$ & -0.30 & & \\
\hline $\mathrm{H}$ & -0.12 & & \\
\hline $\mathrm{H}$ & -0.6 & -0.3 & 27 \\
\hline $\mathrm{H}$ & -0.3 & & \\
\hline $\mathrm{H}$ & -2.0 & -2.2 & -1.5 \\
\hline $\mathrm{H}$ & -2.30 & -1.0 & -2.5 \\
\hline $\mathrm{H}$ & -0.53 & -2.8 & \\
\hline $\mathrm{H}$ & -2.0 & -2.4 & 722 \\
\hline $\mathrm{H}$ & 0.03 & 3.00 & \\
\hline $\mathrm{H}$ & -1.5 & 3.0 & \\
\hline $\mathrm{C}$ & 2.01 & -0.35 & \\
\hline $\mathrm{C}$ & 3.4 & -0.7 & -0 . \\
\hline $\mathrm{N}$ & & & \\
\hline $\mathrm{H}$ & & -1.5 & \\
\hline $\mathrm{H}$ & 3.71 & -1.1 & \\
\hline $\mathrm{H}$ & & & \\
\hline $\mathrm{H}$ & 5.40 & & \\
\hline $\mathrm{H}$ & 4.248 & & -0. \\
\hline \multicolumn{4}{|c|}{$\left[{ }^{1} \mathrm{La}\left(\mathrm{H}_{2} \mathrm{O}\right)_{9}\right]^{3+}$} \\
\hline \multicolumn{4}{|c|}{$E=-1123.1190831$} \\
\hline & & & \\
\hline $\mathrm{O}$ & -1.22 & & \\
\hline $\mathrm{H}$ & -0.81 & & \\
\hline $\mathrm{O}$ & -2.41 & & \\
\hline $\mathrm{H}$ & -3.19 & -0.1 & -0. \\
\hline $\mathrm{O}$ & -1.73 & -1.4 & 3349 \\
\hline $\mathrm{H}$ & -2.01 & -2.3 & \\
\hline $\mathrm{O}$ & 0.000000 & -2.4512 & -0.9 \\
\hline $\mathrm{H}$ & -0.257062 & -2.733377 & 3250 \\
\hline $\mathrm{O}$ & 1.225337 & -1.4708 & \\
\hline $\mathrm{H}$ & 2.158648 & -1.747759 & 6119 \\
\hline $\mathrm{O}$ & 1.739422 & 1.440581 & \\
\hline $\mathrm{H}$ & 2.014450 & 2.358137 & 1.168898 \\
\hline $\mathrm{O}$ & 2.413513 & -0.448854 & -0.915795 \\
\hline $\mathrm{H}$ & 2.736738 & -1.229405 & -1.399797 \\
\hline $\mathrm{O}$ & 0.000000 & 0.000000 & -2.632095 \\
\hline
\end{tabular}




$\begin{array}{rrrr}\mathrm{H} & -0.773131 & 0.054757 & -3.221209 \\ \mathrm{O} & 0.000000 & 2.451231 & -0.907297 \\ \mathrm{H} & -0.283012 & 3.263438 & -0.451029 \\ \mathrm{H} & -2.736738 & 1.229405 & -1.399797 \\ \mathrm{H} & -2.254814 & -1.157548 & 2.119359 \\ \mathrm{H} & 0.283012 & -3.263438 & -0.451029 \\ \mathrm{H} & 3.192734 & 0.123469 & -0.798489 \\ \mathrm{H} & 0.817094 & -1.894237 & 2.567978 \\ \mathrm{H} & -2.158648 & 1.747759 & 1.806119 \\ \mathrm{H} & 0.257062 & 2.733377 & -1.803250 \\ \mathrm{H} & 2.254814 & 1.157548 & 2.119359 \\ \mathrm{H} & 0.773131 & -0.054757 & -3.221209\end{array}$

$\left[(\text { Glycine })^{1} \mathrm{La}\left(\mathrm{H}_{2} \mathrm{O}\right)_{6}\left(\mathrm{OH}^{-}\right)\right]^{+2}$ (Equation 5) $\mathrm{E}=-1178.1131485$

$\begin{array}{lrrr}\text { LA } & -0.774831 & -0.057919 & 0.000904 \\ \mathrm{O} & 1.420341 & 1.341484 & -0.031140 \\ \mathrm{O} & 1.925629 & -0.851616 & 0.012913 \\ \mathrm{C} & 2.239022 & 0.380617 & -0.010934 \\ \mathrm{C} & 3.743272 & 0.715258 & -0.014491 \\ \mathrm{~N} & 4.491213 & -0.601722 & 0.007636 \\ \mathrm{H} & 4.006551 & 1.304983 & 0.863433 \\ \mathrm{H} & 4.009370 & 1.277339 & -0.909491 \\ \mathrm{H} & 5.078031 & -0.741801 & -0.819174 \\ \mathrm{H} & 5.081059 & -0.712472 & 0.836732 \\ \mathrm{H} & 3.726858 & -1.316863 & 0.021504 \\ \mathrm{O} & -0.475631 & -0.708435 & 2.529116 \\ \mathrm{H} & -0.510967 & -1.571736 & 2.968660 \\ \mathrm{H} & -0.700398 & -0.043854 & 3.199793 \\ \mathrm{O} & -1.135266 & -2.673864 & 0.064118 \\ \mathrm{H} & -2.061571 & -2.966500 & 0.080591 \\ \mathrm{H} & -0.589199 & -3.474849 & 0.079110 \\ \mathrm{O} & -1.104407 & 1.915053 & -1.750832 \\ \mathrm{H} & -1.994361 & 2.253951 & -1.940152 \\ \mathrm{H} & -0.487410 & 2.648233 & -1.904154 \\ \mathrm{O} & -0.513951 & -0.836552 & -2.496469 \\ \mathrm{H} & -0.565299 & -1.720142 & -2.891963 \\ \mathrm{H} & -0.727579 & -0.202694 & -3.199663 \\ \mathrm{O} & -1.110684 & 1.996030 & 1.653968 \\ \mathrm{H} & -2.006739 & 2.330877 & 1.820255 \\ \mathrm{H} & -0.503926 & 2.742589 & 1.780807 \\ \mathrm{O} & -2.889298 & -0.037274 & 0.022375 \\ \mathrm{H} & -3.855179 & -0.027500 & 0.028870 \\ & & & \\ {[(\mathrm{Glycine})} & 1 \\ \left.\mathrm{La}\left(\mathrm{H}_{2} \mathrm{O}\right)_{7}\right]^{+3}(\text { Equation } 4) \\ \mathrm{E}=-1178.2740212 & & \end{array}$




$\begin{array}{lccc}\text { LA } & -0.640578 & 0.039116 & -0.031313 \\ \text { O } & 1.875660 & 0.758496 & 0.286887 \\ \text { O } & 1.368231 & -1.284683 & -0.438388 \\ \text { C } & 2.224070 & -0.395388 & -0.109075 \\ \text { C } & 3.700479 & -0.789059 & -0.187065 \\ \text { N } & 4.609758 & 0.387790 & 0.088366 \\ \text { H } & 3.906211 & -1.561428 & 0.558253 \\ \text { H } & 3.937590 & -1.196497 & -1.171787 \\ \text { H } & 4.280176 & 0.924304 & 0.901041 \\ \mathrm{H} & 5.572260 & 0.074716 & 0.273777 \\ \mathrm{H} & 4.647175 & 1.038188 & -0.705539 \\ \mathrm{O} & -0.673753 & 2.480229 & 0.849306 \\ \mathrm{H} & -1.424690 & 3.059526 & 1.071233 \\ \mathrm{H} & 0.127199 & 2.997147 & 1.047829 \\ \mathrm{O} & -1.975754 & -0.928203 & -2.030457 \\ \mathrm{H} & -2.409681 & -0.488739 & -2.782946 \\ \mathrm{H} & -2.108526 & -1.881841 & -2.177009 \\ \mathrm{O} & -3.124897 & 0.281546 & 0.718912 \\ \mathrm{H} & -3.920315 & 0.229273 & 0.158974 \\ \mathrm{H} & -3.460580 & 0.378824 & 1.627854 \\ \mathrm{O} & -0.553386 & -0.236624 & 2.531027 \\ \mathrm{H} & -0.613661 & -1.077488 & 3.017776 \\ \mathrm{H} & -0.365731 & 0.442222 & 3.202727 \\ \mathrm{O} & -1.074993 & -2.477009 & 0.433049 \\ \mathrm{H} & -1.844420 & -3.001676 & 0.716314 \\ \mathrm{H} & -0.348097 & -3.111783 & 0.296750 \\ \mathrm{O} & -0.419872 & 1.584478 & -2.099815 \\ \mathrm{H} & -0.405358 & 2.557289 & -2.067415 \\ \mathrm{H} & -0.242077 & 1.354670 & -3.028898\end{array}$

Geometries of calculated acidities of $\left[\mathrm{Met}(\mathrm{III})\left(\mathrm{H}_{2} \mathrm{O}\right)_{\mathrm{z}}\left(\mathrm{H}_{2} \mathrm{O}\right)_{2 \mathrm{z}}\right]^{3+}$ and $\left[\mathrm{Met}(\mathrm{III})\left(\mathrm{H}_{2} \mathrm{O}\right)_{\mathrm{z}}\right]^{3+}$ complexes (Table 5).

\begin{tabular}{|c|c|c|c|}
\hline \multicolumn{4}{|c|}{$\left[{ }^{1} \mathrm{Al}\left(\mathrm{H}_{2} \mathrm{O}\right)_{6}(\mathrm{H} 2 \mathrm{O})_{12}\right]^{3+}$} \\
\hline AL & 0.000000 & 0.000000 & 0.000006 \\
\hline $\mathrm{O}$ & 0.000000 & 0.000000 & 1.919342 \\
\hline $\mathrm{O}$ & 1.9743 & 0.000004 & 0.000003 \\
\hline $\mathrm{O}$ & 0.0000 & 0.000000 & -1.9 \\
\hline $\mathrm{O}$ & -1.974339 & -0.000004 & 0.000003 \\
\hline $\mathrm{O}$ & 0.000000 & -1.882327 & 0.000000 \\
\hline $\mathrm{O}$ & 0.000000 & 1.882327 & 000 \\
\hline $\mathrm{H}$ & -0.351366 & -2.469252 & -0.742541 \\
\hline $\mathrm{H}$ & 0.351385 & -2.469248 & 0.742534 \\
\hline $\mathrm{H}$ & 2.548398 & -0.585761 & -0.546529 \\
\hline
\end{tabular}




\begin{tabular}{|c|c|c|c|}
\hline $\mathrm{H}$ & 2.548390 & 0.585763 & 0.546550 \\
\hline H & -0.469237 & -0.660625 & 2.500778 \\
\hline & 0.469237 & 0.660625 & 2.500778 \\
\hline $\mathrm{H}$ & -2.548398 & 0.585761 & -0.546529 \\
\hline & -2.548390 & -0.585763 & 0.546550 \\
\hline & 0.469242 & -0.660623 & -2.500762 \\
\hline & -0.469242 & 0.660623 & -2.500762 \\
\hline & 0.351366 & 2.469252 & -0.742541 \\
\hline & -0.351385 & 2.469248 & 0.742534 \\
\hline & 3.612123 & 1.589360 & 1.689210 \\
\hline H & 4.003074 & 2.410002 & 1.342020 \\
\hline 0 & 3.612189 & -1.589215 & -1.689236 \\
\hline $\mathrm{H}$ & 4.003231 & -2.409830 & -1.342086 \\
\hline 0 & -3.612189 & 1.589215 & -1.689236 \\
\hline $\mathrm{H}$ & -4.353500 & 1.118000 & -2.108383 \\
\hline $\mathrm{O}$ & -3.612123 & -1.589360 & 1.689210 \\
\hline $\mathrm{H}$ & -4.003074 & -2.410002 & 1.342020 \\
\hline $\mathrm{H}$ & 4.353495 & 1.118229 & 2.108342 \\
\hline $\mathrm{H}$ & 4.353500 & -1.118000 & -2.108383 \\
\hline $\mathrm{H}$ & -4.003231 & 2.409830 & -1.342086 \\
\hline $\mathrm{H}$ & -4.353495 & -1.118229 & 2.108342 \\
\hline $\mathrm{O}$ & -0.733242 & -3.347108 & -2.012621 \\
\hline $\mathrm{H}$ & -1.122891 & -4.231304 & -2.087765 \\
\hline $\mathrm{O}$ & 0.733401 & -3.347065 & 2.012597 \\
\hline $\mathrm{H}$ & 0.084391 & -3.241553 & 2.731592 \\
\hline $\mathrm{H}$ & -0.084200 & -3.241541 & -2.731580 \\
\hline $\mathrm{H}$ & 1.123129 & -4.231229 & 2.087725 \\
\hline $\mathrm{O}$ & 0.733242 & 3.347108 & -2.012621 \\
\hline $\mathrm{H}$ & 1.122891 & 4.231304 & -2.087765 \\
\hline $\mathrm{O}$ & -0.733401 & 3.347065 & 2.012597 \\
\hline $\mathrm{H}$ & -0.084391 & 3.241553 & 2.731592 \\
\hline $\mathrm{H}$ & 0.084200 & 3.241541 & -2.731580 \\
\hline $\mathrm{H}$ & -1.123129 & 4.231229 & 2.087725 \\
\hline $\mathrm{O}$ & 1.301430 & 1.856276 & 3.374728 \\
\hline $\mathrm{H}$ & 2.207995 & 1.927137 & 3.008786 \\
\hline $\mathrm{O}$ & -1.301430 & -1.856276 & 3.374728 \\
\hline $\mathrm{H}$ & -1.390097 & -1.837970 & 4.342152 \\
\hline $\mathrm{H}$ & 1.390097 & 1.837970 & 4.342152 \\
\hline $\mathrm{H}$ & -2.207995 & -1.927137 & 3.008786 \\
\hline $\mathrm{O}$ & 1.301461 & -1.856261 & -3.374687 \\
\hline $\mathrm{H}$ & 2.208039 & -1.927084 & -3.008773 \\
\hline $\mathrm{O}$ & -1.301461 & 1.856261 & -3.374687 \\
\hline $\mathrm{H}$ & -2.208039 & 1.927084 & -3.008773 \\
\hline $\mathrm{H}$ & 1.390096 & -1.837990 & -4.342114 \\
\hline $\mathrm{H}$ & -1.390096 & 1.837990 & -4.342114 \\
\hline
\end{tabular}




$\begin{array}{cccc}{\left[{ }^{1} \mathrm{Al}\left(\mathrm{H}_{2} \mathrm{O}\right)_{5}(\mathrm{OH})\left(\mathrm{H}_{2} \mathrm{O}\right)_{12}\right]^{2+}(\mathrm{a})} \\ \mathrm{E}=-1618.043036 & \\ \mathrm{AL} & -0.126877 & 0.146170 & 0.041143 \\ \mathrm{O} & -1.315288 & 1.649195 & -0.168637 \\ \mathrm{O} & 0.836828 & 1.190194 & 1.350104 \\ \mathrm{H} & -0.992406 & 2.550461 & 0.125781 \\ \mathrm{H} & -1.812269 & 1.800107 & -1.023166 \\ \mathrm{O} & -1.236568 & -0.790905 & -1.282247 \\ \mathrm{O} & -1.318847 & -0.457874 & 1.465245 \\ \mathrm{O} & 0.905933 & 0.833860 & -1.509997 \\ \mathrm{O} & 1.038664 & -1.215402 & 0.222157 \\ \mathrm{H} & 1.141006 & 0.648098 & 2.134618 \\ \mathrm{H} & 1.382555 & 2.013370 & 1.289010 \\ \mathrm{H} & -0.944749 & -1.694751 & -1.565926 \\ \mathrm{H} & -2.224675 & -0.745059 & -1.298695 \\ \mathrm{H} & -1.133279 & -1.244102 & 2.043105 \\ \mathrm{H} & -2.222620 & -0.105534 & 1.609287 \\ \mathrm{H} & 1.136172 & 0.110210 & -2.138197 \\ \mathrm{H} & 1.746299 & 1.340842 & -1.385545 \\ \mathrm{H} & 0.834137 & -2.126750 & 0.475396 \\ \mathrm{O} & -0.384872 & 4.048603 & 0.462783 \\ \mathrm{H} & -0.775928 & 4.920810 & 0.313040 \\ \mathrm{H} & 0.578054 & 4.163331 & 0.574375 \\ \mathrm{O} & -2.950383 & 2.056813 & -2.191672 \\ \mathrm{H} & -2.934575 & 2.522005 & -3.039887 \\ \mathrm{H} & -3.579041 & 1.316884 & -2.258755 \\ \mathrm{O} & -0.485958 & -2.580054 & 2.870772 \\ \mathrm{H} & -0.941898 & -3.040523 & 3.592188 \\ \mathrm{H} & -0.265970 & -3.257668 & 2.189782 \\ \mathrm{O} & 1.966203 & -1.460611 & -2.829253 \\ \mathrm{H} & 2.362530 & -1.494390 & -3.714231 \\ \mathrm{H} & 2.710332 & -1.438190 & -2.179432 \\ \mathrm{O} & 3.485823 & -0.879337 & -0.599284 \\ \mathrm{H} & 4.173896 & -1.378206 & -0.133561 \\ \mathrm{H} & 2.602629 & -1.046372 & -0.127081 \\ \mathrm{O} & 1.525663 & -0.413916 & 3.360899 \\ \mathrm{H} & 1.744312 & -0.209158 & 4.281405 \\ \mathrm{H} & 1.019602 & -1.247808 & 3.360943 \\ \mathrm{O} & -0.125759 & -3.166652 & -2.043581 \\ \mathrm{H} & -0.472589 & -3.717398 & -2.763261 \\ \mathrm{H} & 0.695186 & -2.736908 & -2.386380 \\ \mathrm{O} & -3.958858 & -0.426122 & -1.222265 \\ \mathrm{H} & -4.644679 & -1.030178 & -1.546504 \\ \mathrm{H} & -4.204492 & -0.175909 & -0.306485 \\ \mathrm{O} & 2.339933 & 3.404102 & 0.967453 \\ \mathrm{H} & 2.875391 & 3.854470 & 1.638933\end{array}$




$\begin{array}{rrrr}\mathrm{H} & 2.956205 & 3.039844 & 0.292818 \\ \mathrm{O} & 0.340421 & -4.048798 & 0.659627 \\ \mathrm{H} & 0.886162 & -4.842819 & 0.763920 \\ \mathrm{H} & 0.087012 & -4.003956 & -0.285642 \\ \mathrm{O} & 3.422881 & 1.820065 & -1.023697 \\ \mathrm{H} & 3.697242 & 0.893375 & -0.798304 \\ \mathrm{H} & 4.027066 & 2.138969 & -1.712465 \\ \mathrm{O} & -3.988559 & 0.509788 & 1.487914 \\ \mathrm{H} & -3.988622 & 1.481658 & 1.476726 \\ \mathrm{H} & -4.646641 & 0.257450 & 2.156783\end{array}$

$\left[{ }^{1} \mathrm{Al}\left(\mathrm{H}_{2} \mathrm{O}\right)_{5}(\mathrm{OH})\left(\mathrm{H}_{2} \mathrm{O}\right)_{12}\right]^{2+}(\mathrm{b})$

$\mathrm{E}=-1618.043038$

$\begin{array}{lrrr}\mathrm{O} & -0.834587 & 1.191225 & -1.350028 \\ \mathrm{H} & -1.140854 & 0.649398 & -2.133936 \\ \mathrm{H} & -1.378133 & 2.015870 & -1.289089 \\ \mathrm{O} & -1.528616 & -0.411890 & -3.359586 \\ \mathrm{H} & -1.744473 & -0.206514 & -4.280616 \\ \mathrm{H} & -1.025514 & -1.247599 & -3.358417 \\ \mathrm{O} & -2.332664 & 3.408455 & -0.967755 \\ \mathrm{H} & -2.867204 & 3.859886 & -1.639248 \\ \mathrm{H} & -2.949647 & 3.045618 & -0.292988 \\ \mathrm{O} & 0.478941 & -2.580828 & -2.871043 \\ \mathrm{H} & 0.258694 & -3.258249 & -2.189899 \\ \mathrm{H} & 0.933127 & -3.041892 & -3.593186 \\ \mathrm{O} & -3.419040 & 1.827347 & 1.023551 \\ \mathrm{H} & -4.022331 & 2.147560 & 1.712501 \\ \mathrm{H} & -3.695094 & 0.901049 & 0.798874 \\ \mathrm{O} & -1.040894 & -1.213463 & -0.221733 \\ \mathrm{H} & -0.837963 & -2.125108 & -0.475174 \\ \mathrm{O} & -3.487026 & -0.872395 & 0.600791 \\ \mathrm{H} & -4.176339 & -1.370411 & 0.135970 \\ \mathrm{H} & -2.604476 & -1.041302 & 0.128033 \\ \mathrm{O} & -0.347438 & -4.048823 & -0.659475 \\ \mathrm{H} & -0.895096 & -4.841586 & -0.763247 \\ \mathrm{H} & -0.094456 & -4.003654 & 0.285933 \\ \mathrm{O} & 0.119813 & -3.167437 & 2.043288 \\ \mathrm{H} & 0.465385 & -3.719270 & 2.762736 \\ \mathrm{H} & -0.700217 & -2.736115 & 2.386208 \\ \mathrm{O} & -1.967690 & -1.456172 & 2.830334 \\ \mathrm{H} & -2.711976 & -1.432321 & 2.180700 \\ \mathrm{H} & -2.363761 & -1.489148 & 3.715442 \\ \mathrm{O} & 1.235286 & -0.793057 & 1.282344 \\ \mathrm{H} & 0.942084 & -1.696434 & 1.565867 \\ \mathrm{H} & 2.223512 & -0.748624 & 1.299261 \\ \mathrm{O} & 3.957342 & -0.432149 & 1.222211\end{array}$




$\begin{array}{cccc}\mathrm{H} & 4.642063 & -1.037254 & 1.546831 \\ \mathrm{H} & 4.203229 & -0.183260 & 0.306114 \\ \mathrm{O} & 3.989099 & 0.501340 & -1.488667 \\ \mathrm{H} & 3.991283 & 1.473213 & -1.478158 \\ \mathrm{H} & 4.647612 & 0.247050 & -2.156373 \\ \mathrm{O} & 1.319107 & 1.646362 & 0.167883 \\ \mathrm{H} & 1.815469 & 1.796963 & 1.022884 \\ \mathrm{H} & 0.998075 & 2.548063 & -0.127286 \\ \mathrm{O} & 0.393689 & 4.047191 & -0.465291 \\ \mathrm{H} & 0.786476 & 4.918621 & -0.315544 \\ \mathrm{H} & -0.569131 & 4.163866 & -0.575840 \\ \mathrm{O} & 2.952703 & 2.052953 & 2.191954 \\ \mathrm{H} & 2.936279 & 2.517055 & 3.040768 \\ \mathrm{H} & 3.580634 & 1.312398 & 2.258938 \\ \mathrm{O} & 1.318118 & -0.461432 & -1.465084 \\ \mathrm{H} & 2.222580 & -0.110984 & -1.609634 \\ \mathrm{H} & 1.129978 & -1.246478 & -2.043703 \\ \mathrm{O} & -0.903625 & 0.836125 & 1.509930 \\ \mathrm{H} & -1.134845 & 0.113236 & 2.138646 \\ \mathrm{H} & -1.743049 & 1.344563 & 1.385497 \\ \mathrm{AL} & 0.127534 & 0.145767 & -0.041373\end{array}$

$\left[{ }^{1} \mathrm{Al}\left(\mathrm{H}_{2} \mathrm{O}\right)_{5}(\mathrm{OH})\left(\mathrm{H}_{2} \mathrm{O}\right)_{12}\right]^{2+}(\mathrm{c})$ $\mathrm{E}=-1618.039803$
$\begin{array}{llll}\text { AL } & -0.075321 & -0.023910 & 0.123886\end{array}$
$\begin{array}{llll}\mathrm{O} & -0.533062 & -0.710159 & -1.618589\end{array}$
$\begin{array}{llll}\mathrm{O} & -1.673061 & -0.838234 & 0.871764\end{array}$
$\begin{array}{llll}\mathrm{H} & 0.200293 & -1.052811 & -2.219494\end{array}$

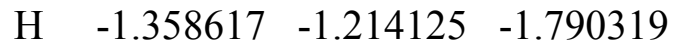
$\begin{array}{llll}\mathrm{O} & 0.176683 & 0.912641 & 1.822588\end{array}$
$\begin{array}{llll}\mathrm{O} & 1.414053 & 0.982535 & -0.654806\end{array}$
$\begin{array}{llll}\mathrm{O} & -1.207864 & 1.512889 & -0.367025\end{array}$
$\begin{array}{llll}\text { O } & 0.978959 & -1.420640 & 0.519288\end{array}$
$\mathrm{H} \quad-1.636522 \quad-1.629871 \quad 1.482553$

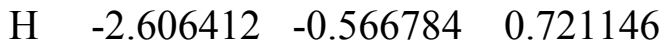
$\begin{array}{llll}\mathrm{H} & 0.179956 & 0.325937 & 2.639585\end{array}$

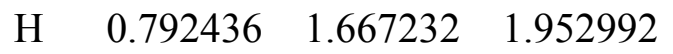
$\begin{array}{llll}\mathrm{H} & 2.244100 & 0.543458 & -0.976251\end{array}$
$\begin{array}{llll}\mathrm{H} & 1.413027 & 1.934828 & -0.900410\end{array}$

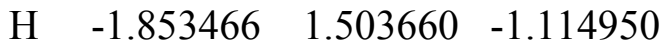
$\begin{array}{llll}\mathrm{H} & -1.270254 & 2.364165 & 0.127418\end{array}$

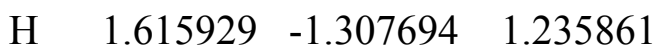
$\begin{array}{llll}\text { O } & 1.444190 & -1.437257 & -3.155898\end{array}$
$\mathrm{H} \quad 1.551770 \quad-2.251517 \quad-3.667015$
$\mathrm{H} \quad 2.307748 \quad-1.218374 \quad-2.747397$
O $\quad 0.244291 \quad-0.860122 \quad 3.752565$ 


$\begin{array}{crrr}\mathrm{H} & 0.271773 & -0.779079 & 4.716434 \\ \mathrm{H} & -0.280545 & -1.661891 & 3.533196 \\ \mathrm{O} & -1.310733 & -2.864378 & 2.552873 \\ \mathrm{H} & -1.993168 & -3.406064 & 2.976937 \\ \mathrm{H} & -0.773948 & -3.467036 & 1.947585 \\ \mathrm{O} & 0.112864 & -3.946508 & 0.674800 \\ \mathrm{H} & 0.761335 & -4.663670 & 0.676475 \\ \mathrm{H} & 0.581775 & -3.088896 & 0.487134 \\ \mathrm{O} & 3.588582 & -0.235332 & -1.776836 \\ \mathrm{H} & 4.116255 & 0.287740 & -2.400447 \\ \mathrm{H} & 4.243874 & -0.752343 & -1.238602 \\ \mathrm{O} & -3.296154 & 1.247994 & -2.130663 \\ \mathrm{H} & -3.620262 & 1.889897 & -2.781232 \\ \mathrm{H} & -3.243503 & 0.380229 & -2.584378 \\ \mathrm{O} & -0.936466 & 4.023054 & 0.711961 \\ \mathrm{H} & -1.618045 & 4.685252 & 0.905162 \\ \mathrm{H} & -0.330376 & 3.998804 & 1.481398 \\ \mathrm{O} & -4.264667 & -0.341058 & 0.021524 \\ \mathrm{H} & -5.082939 & -0.379408 & 0.540521 \\ \mathrm{H} & -4.316776 & 0.455343 & -0.542544 \\ \mathrm{O} & 1.464879 & 3.764624 & -0.798848 \\ \mathrm{H} & 1.900034 & 4.330109 & -1.455627 \\ \mathrm{H} & 0.577160 & 4.143132 & -0.639410 \\ \mathrm{O} & 1.467092 & 3.373405 & 2.016640 \\ \mathrm{H} & 1.994014 & 3.715294 & 2.755565 \\ \mathrm{H} & 1.922651 & 3.629993 & 1.190530 \\ \mathrm{O} & -3.102808 & -1.551586 & -2.279158 \\ \mathrm{H} & -3.341765 & -2.271307 & -2.883752 \\ \mathrm{H} & -3.706674 & -1.605774 & -1.512947 \\ \mathrm{O} & 5.378465 & -1.662421 & -0.309580 \\ \mathrm{H} & 6.090809 & -1.279729 & 0.224216 \\ \mathrm{H} & 5.685580 & -2.543455 & -0.571260\end{array}$

$\left[{ }^{1} \mathrm{Al}\left(\mathrm{H}_{2} \mathrm{O}\right)_{5}(\mathrm{OH})\left(\mathrm{H}_{2} \mathrm{O}\right)_{12}\right]^{2+}(\mathrm{d})$ $\mathrm{E}=-1618.042612$
$\begin{array}{llll}\text { AL } & -0.016913 & -0.265077 & 0.172790\end{array}$
$\begin{array}{llll}\text { O } & 1.145315 & -1.134285 & -1.141905\end{array}$
$\begin{array}{llll}\mathrm{O} & -0.975236 & 0.637602 & 1.567714\end{array}$
$\mathrm{H} \quad 0.695638 \quad-1.318975 \quad-2.027346$
$\mathrm{H} \quad 2.030418 \quad-0.761970 \quad-1.356319$
$\begin{array}{llll}\mathrm{O} & -0.011732 & 1.386312 & -0.896762\end{array}$
$\begin{array}{llll}\text { O } & 1.650671 & 0.402313 & 0.987462\end{array}$
$\begin{array}{llll}\mathrm{O} & -1.527041 & -0.930314 & -0.513077\end{array}$
$\mathrm{H} \quad-1.913839 \quad 0.893565 \quad 1.364009$
$\mathrm{H} \quad-0.584583 \quad 1.363009 \quad 2.124203$
H $\quad-0.868155 \quad 1.781206 \quad-1.209836$ 


\begin{tabular}{|c|c|c|c|}
\hline $\mathrm{H}$ & 0.769469 & 1.843195 & -1.283584 \\
\hline $\mathrm{H}$ & 2.419724 & -0.173132 & 1.210313 \\
\hline $\mathrm{H}$ & 1.901292 & 1.344968 & 1.169295 \\
\hline $\mathrm{H}$ & -1.868716 & -0.782603 & -1.408863 \\
\hline $\mathrm{O}$ & -3.504796 & 1.390197 & 0.889040 \\
\hline $\mathrm{H}$ & -3.924291 & 2.070757 & 1.438484 \\
\hline $\mathrm{H}$ & -4.025149 & 0.545833 & 1.049509 \\
\hline $\mathrm{O}$ & 0.074864 & -1.31 & -3.514874 \\
\hline $\mathrm{H}$ & 0.161172 & -1.99 & -4.197830 \\
\hline $\mathrm{H}$ & -0.826770 & -0.92 & -3.590094 \\
\hline $\mathrm{O}$ & 3.715933 & -0.0 & -1.504173 \\
\hline $\mathrm{H}$ & 4.305523 & -0.33 & -2.222631 \\
\hline $\mathrm{H}$ & 4.087 & -0.4 & -0.6 \\
\hline $\mathrm{O}$ & -0.028156 & 2.7 & 2.82 \\
\hline $\mathrm{H}$ & -0.121 & 3.1 & 205 \\
\hline $\mathrm{H}$ & 0.729 & 3.2 & 2.409083 \\
\hline $\mathrm{O}$ & -4.634 & -0.9 & \\
\hline $\mathrm{H}$ & -5.436 & -1.1 & 1.895351 \\
\hline $\mathrm{H}$ & -3.976 & -1.6 & 1.542794 \\
\hline $\mathrm{O}$ & 2.3 & 2.9 & 1.2 \\
\hline $\mathrm{H}$ & 3.175 & 3.3 & 1.67 \\
\hline $\mathrm{H}$ & $2.50^{7}$ & 3.1 & 0.2 \\
\hline $\mathrm{O}$ & 3.878669 & -1.2 & 1.1 \\
\hline $\mathrm{H}$ & & & \\
\hline $\mathrm{H}$ & 3.5538 & -2.1 & 2090 \\
\hline $\mathrm{O}$ & -2.524424 & 2.1 & -1.5 \\
\hline $\mathrm{H}$ & -2.827 & 2.9 & -1.8 \\
\hline $\mathrm{H}$ & -3.024514 & 1.8 & -0.7 \\
\hline $\mathrm{O}$ & 2.418 & -3.6 & 1.24 \\
\hline $\mathrm{H}$ & 2.519967 & -4.2 & 0.537226 \\
\hline $\mathrm{H}$ & 2.471668 & -4.13 & 4913 \\
\hline $\mathrm{O}$ & 2.441902 & 2.50 & -1.548240 \\
\hline $\mathrm{H}$ & 2.637884 & 3.08 & -2.297641 \\
\hline $\mathrm{H}$ & 3.040721 & 1.72 & -1.625943 \\
\hline $\mathrm{O}$ & -2.467365 & -0.2 & -3.1 \\
\hline $\mathrm{H}$ & -3.266358 & -0.5 & -3.640792 \\
\hline $\mathrm{H}$ & -2.643833 & 0.6 & -2.870040 \\
\hline $\mathrm{O}$ & -2.388578 & -2.651498 & 1.193816 \\
\hline $\mathrm{H}$ & -2.222182 & -2.1 & 0.362840 \\
\hline $\mathrm{H}$ & -2.617190 & -3.553825 & 0.924443 \\
\hline $\mathrm{O}$ & 0.173048 & -1.816633 & 1.373442 \\
\hline $\mathrm{H}$ & 0.838554 & -2.512632 & 1.198516 \\
\hline $\mathrm{H}$ & -0.708259 & -2.260415 & 1.523961 \\
\hline
\end{tabular}




\begin{tabular}{|c|c|c|c|}
\hline $\mathrm{AL}$ & -0.148578 & 0.017269 & 0.014044 \\
\hline $\mathrm{O}$ & -0.429731 & 1.117015 & 1.603751 \\
\hline $\mathrm{O}$ & -0.170195 & -1.103133 & -1.566820 \\
\hline $\mathrm{H}$ & 0.180829 & 0.912792 & 2.376508 \\
\hline $\mathrm{H}$ & -0.620746 & 2.081252 & 1.583385 \\
\hline $\mathrm{O}$ & -0.198897 & -1.561949 & 1.156185 \\
\hline $\mathrm{O}$ & -0.141152 & 1.597183 & -1.132047 \\
\hline $\mathrm{O}$ & 1.641376 & 0.051354 & 0.091189 \\
\hline $\mathrm{H}$ & 0.528305 & -0.899467 & -2.267317 \\
\hline $\mathrm{H}$ & -0.313830 & -2.073941 & -1.531399 \\
\hline $\mathrm{H}$ & 0.638265 & -1.861787 & 1.597952 \\
\hline $\mathrm{H}$ & -0.933937 & -2.200274 & 1.304606 \\
\hline $\mathrm{H}$ & 0.734512 & 1.893901 & -1.494760 \\
\hline $\mathrm{H}$ & -0.863080 & 2.232859 & -1.343473 \\
\hline $\mathrm{H}$ & 2.060492 & 0.803479 & 0.531467 \\
\hline $\mathrm{O}$ & 1.734728 & -0.495329 & -3.238268 \\
\hline $\mathrm{H}$ & 1.767391 & -0.602824 & -4.19 \\
\hline $\mathrm{H}$ & 2.082757 & 0.397665 & -3.024106 \\
\hline $\mathrm{O}$ & -1.488803 & 3.685911 & 1.499681 \\
\hline $\mathrm{H}$ & -1.313115 & 4.412551 & 2.11 \\
\hline $\mathrm{H}$ & -1.515965 & 4.068807 & 0.600857 \\
\hline $\mathrm{O}$ & 1.344567 & 0.476272 & 3.42 \\
\hline $\mathrm{H}$ & 1.454894 & 0.696673 & 4.356727 \\
\hline $\mathrm{H}$ & 1.767245 & -0.394289 & 3.261712 \\
\hline $\mathrm{O}$ & -1.133277 & -3.727598 & -1.528766 \\
\hline $\mathrm{H}$ & -0.857890 & -4.458045 & -2.104539 \\
\hline $\mathrm{H}$ & -1.266572 & -4.097560 & -0.63 \\
\hline $\mathrm{O}$ & 2.186701 & -2.044026 & 2.416459 \\
\hline $\mathrm{H}$ & 2.444794 & -2.792453 & 2.976226 \\
\hline $\mathrm{H}$ & 2.920248 & -1.907544 & 1.759784 \\
\hline $\mathrm{O}$ & 3.809698 & -1.342636 & 0.321260 \\
\hline $\mathrm{H}$ & 4.126795 & -1.981635 & -0.334007 \\
\hline $\mathrm{H}$ & 2.944996 & -0.952060 & 0.000621 \\
\hline $\mathrm{O}$ & -2.193312 & -3.471556 & 1.098579 \\
\hline $\mathrm{H}$ & -2.505021 & -4.028218 & 1.829345 \\
\hline $\mathrm{H}$ & -2.985638 & -3.122612 & 0.644767 \\
\hline $\mathrm{O}$ & 2.361779 & 2.039034 & -2.159832 \\
\hline $\mathrm{H}$ & 2.656216 & 2.794901 & -2.691058 \\
\hline $\mathrm{H}$ & 3.084806 & 1.859830 & -1.500071 \\
\hline $\mathrm{O}$ & 4.248948 & 1.454792 & -0.261696 \\
\hline $\mathrm{H}$ & 5.049866 & 1.958807 & -0.056070 \\
\hline $\mathrm{H}$ & 4.437575 & 0.517348 & -0.048590 \\
\hline $\mathrm{O}$ & -2.179157 & 3.457406 & -1.249368 \\
\hline $\mathrm{H}$ & -2.419225 & 4.028760 & -1.995714 \\
\hline $\mathrm{H}$ & -3.007593 & 3.064589 & -0.910046 \\
\hline $\mathrm{O}$ & -3.647091 & 2.047495 & 0.766208 \\
\hline
\end{tabular}




$\begin{array}{crrc}\mathrm{H} & -3.145316 & 2.632610 & 1.371693 \\ \mathrm{H} & -4.556671 & 2.004739 & 1.100401 \\ \mathrm{O} & -3.433277 & -2.173944 & -1.132974 \\ \mathrm{H} & -4.285551 & -2.177885 & -1.595931 \\ \mathrm{H} & -2.824445 & -2.747791 & -1.644123 \\ \mathrm{O} & -2.091125 & -0.031413 & -0.098581 \\ \mathrm{H} & -2.698873 & 0.609479 & 0.345310 \\ \mathrm{H} & -2.609229 & -0.696198 & -0.615455\end{array}$

$\left[{ }^{1} \mathrm{Al}\left(\mathrm{H}_{2} \mathrm{O}\right)_{5}(\mathrm{OH})\left(\mathrm{H}_{2} \mathrm{O}\right)_{12}\right]^{2+}(\mathrm{f})$ $\mathrm{E}=-1618.042612$
AL $\quad-0.016985 \quad-0.265580 \quad 0.172436$
$\begin{array}{lllll}\text { O } & 1.146147 & -1.131882 & -1.143580\end{array}$
$\begin{array}{llll}\mathrm{O} & -0.975826 & 0.634646 & 1.568635\end{array}$
$\mathrm{H} \quad 0.696944 \quad-1.313959 \quad-2.029844$
$\mathrm{H} \quad 2.031597 \quad-0.759472 \quad-1.356495$
$\begin{array}{llll}\mathrm{O} & 1.650092 & 0.400520 & 0.989464\end{array}$
$\begin{array}{llll}\mathrm{O} & 0.172957 & -1.819355 & 1.370387\end{array}$
$\begin{array}{lllll}\text { O } & -0.011634 & 1.387768 & -0.894087\end{array}$
$\begin{array}{llll}\mathrm{O} & -1.526699 & -0.930082 & -0.514864\end{array}$
$\mathrm{H} \quad-1.914604 \quad 0.890481 \quad 1.365370$
$\mathrm{H} \quad-0.586090 \quad 1.358537 \quad 2.127832$
H $1.900733 \quad 1.343057 \quad 1.171979$
H $\quad 2.419231 \quad-0.175057 \quad 1.211534$
$\mathrm{H} \quad-0.708243 \quad-2.263591 \quad 1.519776$
$\mathrm{H} \quad 0.838495 \quad-2.514935 \quad 1.193911$
$\mathrm{H} \quad-0.867935 \quad 1.783398 \quad-1.206540$
$\mathrm{H} \quad 0.769571 \quad 1.844585 \quad-1.281048$
$\mathrm{H} \quad-1.868310 \quad-0.780398 \quad-1.410345$
$\begin{array}{llll}\text { O } & -3.505126 & 1.387975 & 0.891520\end{array}$
$\mathrm{H} \quad-3.924234 \quad 2.067495 \quad 1.442544$
$\begin{array}{llll}\mathrm{H} & -4.025648 & 0.543394 & 1.050353\end{array}$
O $\quad 0.076413 \quad-1.304423 \quad-3.517268$
$\mathrm{H} \quad 0.162922 \quad-1.982868 \quad-4.201801$
$\mathrm{H} \quad-0.825790 \quad-0.921040 \quad-3.590634$
$\begin{array}{llll}\mathrm{O} & -4.635252 & -0.940629 & 1.401338\end{array}$
$\mathrm{H} \quad-5.436952 \quad-1.169281 \quad 1.892033$
H $\quad-3.977449 \quad-1.654354 \quad 1.539312$
O $\quad 3.716780 \quad-0.054638-1.503262$
$\mathrm{H} \quad 4.306450 \quad-0.334845 \quad-2.221704$
H $\quad 4.088099 \quad-0.424927 \quad-0.674103$
$\begin{array}{llll}\text { O } & -0.029118 & 2.779492 & 2.826746\end{array}$
$\mathrm{H} \quad-0.120942 \quad 3.115697 \quad 3.729640$
$\mathrm{H} \quad 0.729474 \quad 3.227513 \quad 2.413364$
$\begin{array}{llll}\text { O } & 2.390269 & 2.994654 & 1.229029\end{array}$
H $\quad 3.174089 \quad 3.348019 \quad 1.677888$ 


$\begin{array}{lrrr}\mathrm{H} & 2.505887 & 3.174024 & 0.271384 \\ \mathrm{O} & 3.878923 & -1.209238 & 1.105526 \\ \mathrm{H} & 4.606865 & -1.133550 & 1.743050 \\ \mathrm{H} & 3.554520 & -2.136550 & 1.160930 \\ \mathrm{O} & -2.388934 & -2.654917 & 1.188447 \\ \mathrm{H} & -2.618061 & -3.556641 & 0.917546 \\ \mathrm{H} & -2.222049 & -2.105302 & 0.358575 \\ \mathrm{O} & -2.466712 & -0.217836 & -3.183800 \\ \mathrm{H} & -3.265040 & -0.518707 & -3.643210 \\ \mathrm{H} & -2.643569 & 0.692532 & -2.867692 \\ \mathrm{O} & -2.524558 & 2.118492 & -1.584706 \\ \mathrm{H} & -2.828225 & 2.997056 & -1.861564 \\ \mathrm{H} & -3.024850 & 1.888297 & -0.759211 \\ \mathrm{O} & 2.418342 & -3.631845 & 1.239416 \\ \mathrm{H} & 2.520621 & -4.285260 & 0.527597 \\ \mathrm{H} & 2.471517 & -4.141414 & 2.065765 \\ \mathrm{O} & 2.441845 & 2.507024 & -1.544179 \\ \mathrm{H} & 2.637456 & 3.092936 & -2.292717 \\ \mathrm{H} & 3.041047 & 1.731855 & -1.623240\end{array}$

\begin{tabular}{|c|c|c|c|}
\hline \multicolumn{4}{|c|}{$\left[{ }^{1} \mathrm{Al}\left(\mathrm{H}_{2} \mathrm{O}\right)_{6}\right]^{3+}$} \\
\hline $\mathrm{AL}$ & 0.000000 & 0.000000 & 0.000000 \\
\hline $\mathrm{O}$ & 0.000000 & 0.000000 & 1.941523 \\
\hline $\mathrm{O}$ & 0.000000 & 0.000000 & -1.941523 \\
\hline $\mathrm{H}$ & 0.000000 & -0.788072 & 2.524751 \\
\hline $\mathrm{H}$ & 0.000000 & 0.788072 & 2.524751 \\
\hline $\mathrm{O}$ & 0.000000 & 1.941523 & 0.000000 \\
\hline $\mathrm{O}$ & 0.000000 & -1.941523 & 0.000000 \\
\hline $\mathrm{O}$ & 1.941523 & 0.000000 & 0.000000 \\
\hline $\mathrm{O}$ & -1.941523 & 0.000000 & 0.000000 \\
\hline $\mathrm{H}$ & 0.000000 & 0.788072 & -2.524751 \\
\hline $\mathrm{H}$ & 0.000000 & -0.788072 & -2.524751 \\
\hline $\mathrm{H}$ & 0.788072 & 2.524751 & 0.000000 \\
\hline $\mathrm{H}$ & -0.788072 & 2.524751 & 0.000000 \\
\hline $\mathrm{H}$ & -0.788072 & -2.524751 & 0.000000 \\
\hline $\mathrm{H}$ & 0.788072 & -2.524751 & 0.000000 \\
\hline $\mathrm{H}$ & 2.524751 & 0.000000 & -0.788072 \\
\hline $\mathrm{H}$ & 2.524751 & 0.000000 & 0.788072 \\
\hline $\mathrm{H}$ & -2.524751 & 0.000000 & 0.788072 \\
\hline $\mathrm{H}$ & -2.524751 & 0.000000 & -0.788072 \\
\hline
\end{tabular}

$\left[{ }^{1} \mathrm{Al}\left(\mathrm{H}_{2} \mathrm{O}\right)_{5}(\mathrm{OH})\right]^{2+}(\mathrm{a})$

$\mathrm{E}=-700.190103$

$\begin{array}{llll}\text { AL } & -0.043038 & -0.007092 & -0.120976 \\ \text { O } & 0.234635 & -1.961211 & -0.088048\end{array}$ 


$\begin{array}{crcc}\mathrm{O} & -0.069259 & 1.970833 & -0.062392 \\ \mathrm{H} & -0.165641 & -2.434044 & -0.842704 \\ \mathrm{H} & 0.455271 & -2.602928 & 0.609815 \\ \mathrm{O} & 0.565950 & 0.031834 & 1.773486 \\ \mathrm{O} & -1.868808 & -0.114136 & 0.620224 \\ \mathrm{O} & 1.905646 & 0.146502 & -0.500898 \\ \mathrm{O} & -0.777889 & -0.046762 & -1.675079 \\ \mathrm{H} & -0.539302 & 2.380842 & -0.813489 \\ \mathrm{H} & -0.066662 & 2.601781 & 0.679119 \\ \mathrm{H} & 0.034518 & 0.179986 & 2.576180 \\ \mathrm{H} & 1.507907 & 0.054186 & 2.023663 \\ \mathrm{H} & -2.283523 & -0.685080 & 1.289649 \\ \mathrm{H} & -2.486208 & -0.048714 & -0.137656 \\ \mathrm{H} & 2.392333 & -0.577609 & -0.936324 \\ \mathrm{H} & 2.284799 & 0.985938 & -0.821379 \\ \mathrm{H} & -0.496186 & 0.021347 & -2.592530\end{array}$

$$
\begin{array}{cccc}
{\left[{ }^{1} \mathrm{Al}\left(\mathrm{H}_{2} \mathrm{O}\right)_{5}(\mathrm{OH})\right]^{2+}(\mathrm{b})} \\
\mathrm{E}=-700.189447 \\
\mathrm{O} & 1.972610 & 0.022462 & -0.057970 \\
\mathrm{H} & 2.433899 & -0.138678 & -0.903198 \\
\mathrm{H} & 2.625531 & 0.093850 & 0.660917 \\
\mathrm{O} & -0.014140 & 1.993952 & -0.083599 \\
\mathrm{H} & 0.764801 & 2.509919 & -0.362324 \\
\mathrm{H} & -0.800119 & 2.499421 & -0.361786 \\
\mathrm{O} & -0.004287 & 0.196101 & 1.823549 \\
\mathrm{H} & -0.000454 & -0.545842 & 2.455264 \\
\mathrm{H} & -0.009951 & 1.037336 & 2.314204 \\
\mathrm{O} & -1.972799 & -0.005779 & -0.063125 \\
\mathrm{H} & -2.627263 & 0.054940 & 0.655355 \\
\mathrm{H} & -2.431133 & -0.173208 & -0.908710 \\
\mathrm{O} & 0.013429 & -1.939720 & 0.332849 \\
\mathrm{H} & -0.756368 & -2.472025 & 0.057067 \\
\mathrm{H} & 0.794998 & -2.459649 & 0.066792 \\
\mathrm{AL} & 0.000065 & -0.008750 & -0.151863 \\
\mathrm{O} & 0.005703 & -0.325483 & -1.832443 \\
\mathrm{H} & 0.001092 & 0.175424 & -2.653455
\end{array}
$$

$$
\begin{aligned}
& {\left[{ }^{1} \mathrm{Al}\left(\mathrm{H}_{2} \mathrm{O}\right)_{5}(\mathrm{OH})\right]^{2+}(\mathrm{c})} \\
& \mathrm{E}=-700.1894467 \\
& \begin{array}{llll}
\text { O } & 0.004502 & -1.993738 & -0.084621
\end{array} \\
& \mathrm{H} \quad 0.788630 \quad-2.502766-0.361538 \\
& \begin{array}{llll}
\mathrm{H} & -0.776369 & -2.506160 & -0.364452
\end{array} \\
& \begin{array}{llll}
\mathrm{O} & 1.972625 & -0.004330 & -0.059678
\end{array}
\end{aligned}
$$

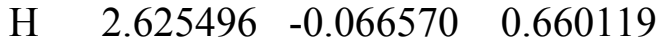

$$
\begin{aligned}
& \begin{array}{llll}
\mathrm{H} & 2.433008 & 0.161488 & -0.904472
\end{array}
\end{aligned}
$$




$\begin{array}{lccl}\mathrm{O} & -0.000275 & -0.196600 & 1.823617 \\ \mathrm{H} & 0.001302 & -1.037851 & 2.314276 \\ \mathrm{H} & 0.001303 & 0.545328 & 2.455363 \\ \mathrm{O} & -1.972627 & -0.013203 & -0.061262 \\ \mathrm{H} & -2.432425 & 0.150629 & -0.906781 \\ \mathrm{H} & -2.626552 & -0.083149 & 0.656856 \\ \mathrm{O} & -0.004338 & 1.939634 & 0.333423 \\ \mathrm{H} & -0.785256 & 2.462748 & 0.071802 \\ \mathrm{H} & 0.765988 & 2.468722 & 0.052898 \\ \mathrm{AL} & 0.000209 & 0.008726 & -0.151862 \\ \mathrm{O} & 0.000157 & 0.326723 & -1.832265 \\ \mathrm{H} & 0.001808 & -0.173747 & -2.653562\end{array}$

$\left[{ }^{1} \mathrm{Al}\left(\mathrm{H}_{2} \mathrm{O}\right)_{5}(\mathrm{OH})\right]^{2+}(\mathrm{d})$

$\mathrm{E}=-700.1894464$

$\begin{array}{lccc}\mathrm{O} & 0.004963 & 0.197280 & 1.823648 \\ \mathrm{H} & 0.013584 & 1.038707 & 2.313925 \\ \mathrm{H} & -0.000177 & -0.544336 & 2.455738 \\ \mathrm{O} & 0.020051 & 1.993703 & -0.084999 \\ \mathrm{H} & -0.757090 & 2.511910 & -0.364572 \\ \mathrm{H} & 0.807842 & 2.496547 & -0.362813 \\ \mathrm{O} & 1.972592 & -0.011454 & -0.063157 \\ \mathrm{H} & 2.430563 & -0.180759 & -0.908571 \\ \mathrm{H} & 2.627067 & 0.046931 & 0.655514 \\ \mathrm{O} & -1.972387 & 0.028417 & -0.057896 \\ \mathrm{H} & -2.624814 & 0.103018 & 0.661120 \\ \mathrm{H} & -2.434470 & -0.132096 & -0.902813 \\ \mathrm{O} & -0.019197 & -1.939352 & 0.334017 \\ \mathrm{H} & 0.748171 & -2.474209 & 0.056420 \\ \mathrm{H} & -0.803122 & -2.456776 & 0.070068 \\ \mathrm{AL} & -0.000145 & -0.008817 & -0.151938 \\ \mathrm{O} & -0.006647 & -0.327068 & -1.832277 \\ \mathrm{H} & -0.000671 & 0.173482 & -2.653512\end{array}$

\begin{tabular}{|c|c|c|c|}
\hline \\
\hline & & & \\
\hline \multirow{2}{*}{\multicolumn{4}{|c|}{$\begin{array}{lll}\mathrm{O} & -0.229115 & 1.961868 \\
\mathrm{H} & -0.448562 & 2.603280\end{array}$}} \\
\hline & & & \\
\hline \multicolumn{4}{|c|}{0.173261} \\
\hline $\mathrm{O}$ & -0.566536 & $\begin{array}{l}2 . \\
-0 .\end{array}$ & 1.773286 \\
\hline \multirow{2}{*}{$\begin{array}{l}\mathrm{H} \\
\mathrm{H}\end{array}$} & -0.035913 & -0.184553 & 2.576117 \\
\hline & -1.508653 & -0.053115 & 2.023159 \\
\hline \multirow{2}{*}{$\begin{array}{l}\mathrm{O} \\
\mathrm{H}\end{array}$} & -1.906012 & -0.139991 & -0.501455 \\
\hline & -2.288555 & -0.977836 & -0.822060 \\
\hline \multirow{2}{*}{$\begin{array}{l}\mathrm{H} \\
\mathrm{O}\end{array}$} & -2.389345 & 0.586153 & -0.937247 \\
\hline & 0.063607 & -1.971055 & -0.066369 \\
\hline
\end{tabular}




\begin{tabular}{cccc}
$\mathrm{H}$ & 0.532451 & -2.381239 & -0.818114 \\
$\mathrm{H}$ & 0.060348 & -2.602834 & 0.674439 \\
$\mathrm{O}$ & 1.869103 & 0.107474 & 0.620720 \\
$\mathrm{H}$ & 2.285163 & 0.675086 & 1.292143 \\
$\mathrm{H}$ & 2.486648 & 0.042313 & -0.137051 \\
$\mathrm{AL}$ & 0.043174 & 0.007088 & -0.120866 \\
$\mathrm{O}$ & 0.778358 & 0.049219 & -1.674793 \\
$\mathrm{H}$ & 0.496650 & -0.019526 & -2.592202 \\
\multicolumn{4}{|l}{} \\
$\left.\mathrm{H}{ }^{1} \mathrm{Al}\left(\mathrm{H}_{2} \mathrm{O}\right)_{5}(\mathrm{OH})\right]^{2+}(\mathrm{f})$ \\
$\mathrm{E}=-700.1894466$ & \\
$\mathrm{O}$ & -0.014906 & 1.939404 & 0.333807 \\
$\mathrm{H}$ & 0.752292 & 2.472819 & 0.052933 \\
$\mathrm{H}$ & -0.799009 & 2.458234 & 0.073223 \\
$\mathrm{O}$ & 1.972657 & 0.006536 & -0.058317 \\
$\mathrm{H}$ & 2.432416 & 0.174510 & -0.903035 \\
$\mathrm{H}$ & 2.625683 & -0.052009 & 0.661649 \\
$\mathrm{O}$ & -0.000578 & -0.197304 & 1.823672 \\
$\mathrm{H}$ & 0.005112 & -1.038797 & 2.313879 \\
$\mathrm{H}$ & -0.003704 & 0.544248 & 2.455849 \\
$\mathrm{O}$ & 0.015185 & -1.993755 & -0.084903 \\
$\mathrm{H}$ & -0.762197 & -2.510464 & -0.366572 \\
$\mathrm{H}$ & 0.802724 & -2.498313 & -0.360338 \\
$\mathrm{O}$ & -1.972381 & -0.023160 & -0.062569 \\
$\mathrm{H}$ & -2.626171 & -0.097659 & 0.655225 \\
$\mathrm{H}$ & -2.432842 & 0.138241 & -0.908193 \\
$\mathrm{AL}$ & 0.000118 & 0.008863 & -0.151974 \\
$\mathrm{O}$ & -0.000046 & 0.326753 & -1.832363 \\
$\mathrm{H}$ & 0.004715 & -0.173829 & -2.653581
\end{tabular}

$\left[{ }^{1} \mathrm{Al}\left(\mathrm{H}_{2} \mathrm{O}\right)_{5}\right]^{3+}$

$\mathrm{E}=-623.7093638$

$\begin{array}{lrrr}\mathrm{AL} & 0.000000 & 0.000000 & 0.038580 \\ \mathrm{O} & -1.742093 & 0.000000 & 0.729502 \\ \mathrm{O} & 1.742093 & 0.000000 & 0.729502 \\ \mathrm{H} & -2.290893 & 0.790563 & 0.934528 \\ \mathrm{H} & -2.290893 & -0.790563 & 0.934528 \\ \mathrm{O} & 0.000000 & 0.000000 & -1.838074 \\ \mathrm{O} & 0.000000 & 1.927042 & 0.176674 \\ \mathrm{O} & 0.000000 & -1.927042 & 0.176674 \\ \mathrm{H} & 2.290893 & -0.790563 & 0.934528 \\ \mathrm{H} & 2.290893 & 0.790563 & 0.934528 \\ \mathrm{H} & -0.791711 & 0.000000 & -2.425276 \\ \mathrm{H} & 0.791711 & 0.000000 & -2.425276 \\ \mathrm{H} & 0.000000 & 2.479601 & 0.990951 \\ \mathrm{H} & 0.000000 & 2.552678 & -0.582612\end{array}$




$$
\begin{array}{lrrr}
\mathrm{H} & 0.000000 & -2.479601 & 0.990951 \\
\mathrm{H} & 0.000000 & -2.552678 & -0.582612 \\
& \\
\left.\left[{ }^{1} \mathrm{Al}\left(\mathrm{H}_{2} \mathrm{O}\right)\right)_{4}(\mathrm{OH})\right]^{2+} \\
\mathrm{E}=-623.6813087
\end{array}
$$

$\begin{array}{lrrr}\mathrm{AL} & -0.004789 & -0.121052 & -0.087390 \\ \mathrm{O} & -0.316530 & -1.492012 & -1.002022 \\ \mathrm{O} & 0.070130 & 1.707888 & -0.595947 \\ \mathrm{H} & 0.123536 & -2.213475 & -1.462801 \\ \mathrm{O} & 0.157965 & -0.220757 & 1.821413 \\ \mathrm{O} & -1.946589 & 0.144087 & -0.007255 \\ \mathrm{O} & 1.972477 & -0.066591 & -0.218100 \\ \mathrm{H} & -0.705497 & 2.191203 & -0.943092 \\ \mathrm{H} & 0.881800 & 2.174046 & -0.875603 \\ \mathrm{H} & 0.075365 & -1.081097 & 2.281207 \\ \mathrm{H} & 0.125813 & 0.487560 & 2.492960 \\ \mathrm{H} & -2.575079 & 0.516102 & 0.637115 \\ \mathrm{H} & -2.397522 & -0.558278 & -0.521529 \\ \mathrm{H} & 2.407102 & -0.533669 & -0.958299 \\ \mathrm{H} & 2.627115 & 0.010368 & 0.501404\end{array}$

$$
\begin{aligned}
& {\left[{ }^{1} \mathrm{Al}\left(\mathrm{H}_{2} \mathrm{O}\right)_{4}\right]^{3+}} \\
& \mathrm{E}=-547.1598716 \\
& \begin{array}{llll}
\text { AL } & 0.000000 & 0.000000 & 0.000000
\end{array} \\
& \begin{array}{llll}
\mathrm{O} & 0.000000 & 1.480411 & 1.081572
\end{array} \\
& \begin{array}{llll}
\mathrm{O} & 1.480411 & 0.000000 & -1.081572
\end{array} \\
& \mathrm{H} \quad-0.553141 \quad 2.293745 \quad 0.964300 \\
& \mathrm{H} \quad 0.543462 \quad 1.624957 \quad 1.896705 \\
& \text { O } \quad-1.480411 \quad 0.000000 \quad-1.081572 \\
& \begin{array}{llll}
\mathrm{O} & 0.000000 & -1.480411 & 1.081572
\end{array} \\
& \mathrm{H} \quad 2.293745 \quad 0.553141 \quad-0.964300 \\
& \mathrm{H} \quad 1.624957 \quad-0.543462-1.896705 \\
& \mathrm{H} \quad-2.293745 \quad-0.553141 \quad-0.964300 \\
& \mathrm{H} \quad-1.624957 \quad 0.543462 \quad-1.896705 \\
& \mathrm{H} \quad 0.553141 \quad-2.293745 \quad 0.964300 \\
& \mathrm{H} \quad-0.543462 \quad-1.624957 \quad 1.896705
\end{aligned}
$$

$\left[{ }^{1} \mathrm{Al}\left(\mathrm{H}_{2} \mathrm{O}\right)_{3}(\mathrm{OH})\right]^{2+}$

$\mathrm{E}=-547.1655055$
AL $\quad 0.163542 \quad-0.022317 \quad 0.000000$
$\begin{array}{llll}\mathrm{O} & -0.604610 & 0.739981 & 1.514993\end{array}$
$\begin{array}{llll}\mathrm{O} & -0.604610 & 0.739981 & -1.514993\end{array}$
$\begin{array}{llll}\mathrm{H} & -0.017892 & 1.247699 & 2.118281\end{array}$
$\mathrm{H} \quad-1.530592 \quad 0.849390 \quad 1.811994$
$\begin{array}{llll}\mathrm{O} & -0.604610 & -1.727709 & 0.000000\end{array}$
$\begin{array}{llll}\text { O } & 1.765808 & 0.332893 & 0.000000\end{array}$ 


$$
\begin{array}{lrrr}
\mathrm{H} & -0.017892 & 1.247699 & -2.118281 \\
\mathrm{H} & -1.530592 & 0.849390 & -1.811994 \\
\mathrm{H} & -0.662363 & -2.306099 & -0.790589 \\
\mathrm{H} & -0.662363 & -2.306099 & 0.790589 \\
\mathrm{H} & 2.679825 & 0.026977 & 0.000000
\end{array}
$$

$\left[{ }^{1} \mathrm{Al}\left(\mathrm{H}_{2} \mathrm{O}\right)_{3}\right]^{3+}$

$\mathrm{E}=-470.5472209$

$\begin{array}{lrrc}\mathrm{AL} & 0.000000 & 0.000000 & 0.000000 \\ \mathrm{O} & 0.000000 & 1.800588 & 0.000000 \\ \mathrm{O} & -1.559355 & -0.900294 & 0.000000 \\ \mathrm{H} & 0.640352 & 2.397347 & 0.480447 \\ \mathrm{H} & -0.640352 & 2.397347 & -0.480447 \\ \mathrm{O} & 1.559355 & -0.900294 & 0.000000 \\ \mathrm{H} & -1.755988 & -1.753235 & -0.480447 \\ \mathrm{H} & -2.396340 & -0.644112 & 0.480447 \\ \mathrm{H} & 1.755988 & -1.753235 & 0.480447 \\ \mathrm{H} & 2.396340 & -0.644112 & -0.480447\end{array}$

$\left[{ }^{1} \mathrm{Al}\left(\mathrm{H}_{2} \mathrm{O}\right)_{2}(\mathrm{OH})\right]^{2+}$

$\mathrm{E}=-470.5989909$

$\begin{array}{lrrr}\mathrm{AL} & 0.000000 & 0.145497 & 0.000000 \\ \mathrm{O} & -1.165567 & -1.257093 & 0.000000 \\ \mathrm{O} & 1.753128 & -0.376746 & 0.000000 \\ \mathrm{H} & -2.135285 & -1.065615 & 0.000000 \\ \mathrm{H} & -1.042135 & -2.232401 & 0.000000 \\ \mathrm{O} & -0.691663 & 1.610582 & 0.000000 \\ \mathrm{H} & 2.483651 & 0.288311 & 0.000000 \\ \mathrm{H} & 2.160919 & -1.272765 & 0.000000 \\ \mathrm{H} & -0.634336 & 2.577065 & 0.000000\end{array}$

$\left[{ }^{1} \mathrm{Al}\left(\mathrm{H}_{2} \mathrm{O}\right)_{2}\right]^{3+}$

$\mathrm{E}=-393.8893089$
$\begin{array}{llll}\mathrm{AL} & 0.000000 & 0.000000 & 0.000000\end{array}$
$\begin{array}{lllll}\mathrm{O} & 0.000000 & 0.000000 & 1.775791\end{array}$
$\begin{array}{lllll}\mathrm{O} & 0.000000 & 0.000000 & -1.775791\end{array}$
$\begin{array}{llll}\mathrm{H} & 0.000000 & 0.811578 & 2.376735\end{array}$
$\begin{array}{llll}\mathrm{H} & 0.000000 & -0.811578 & 2.376735\end{array}$
$\begin{array}{llll}\mathrm{H} & -0.811578 & 0.000000 & -2.376735\end{array}$
$\begin{array}{llll}\mathrm{H} & 0.811578 & 0.000000 & -2.376735\end{array}$

$\left[{ }^{1} \mathrm{Al}\left(\mathrm{H}_{2} \mathrm{O}\right)(\mathrm{OH})\right]^{2+}$

$\mathrm{E}=-393.9965732$
$\begin{array}{llll}\text { AL } & 0.000000 & 0.170552 & 0.000000\end{array}$
$\begin{array}{llll}\mathrm{O} & -1.141105 & -1.223762 & 0.000000\end{array}$
$\begin{array}{llll}\mathrm{O} & 1.320055 & 1.110522 & 0.000000\end{array}$ 


$$
\begin{array}{crcc}
\mathrm{H} & -1.502389 & -1.683340 & 0.804115 \\
\mathrm{H} & -1.502389 & -1.683340 & -0.804115 \\
\mathrm{H} & 1.573173 & 2.055431 & 0.000000
\end{array}
$$

$\left[{ }^{1} \mathrm{Al}\left(\mathrm{H}_{2} \mathrm{O}\right)\right]^{3+}$

$\mathrm{E}=-317.1668042$
$\begin{array}{llll}\text { AL } & 0.000000 & 0.000000 & 0.823160\end{array}$
$\begin{array}{lllll}\mathrm{O} & 0.000000 & 0.000000 & -0.950203\end{array}$

\begin{tabular}{|c|c|c|c|}
\hline $\begin{array}{l}{\left[{ }^{1} \mathrm{~A}\right]} \\
\mathrm{E}=\end{array}$ & $\begin{array}{l}\mathrm{DH})]^{2+} \\
17.3476452\end{array}$ & & \\
\hline $\mathrm{AL}$ & 0.037062 & -0.710591 & 0.000000 \\
\hline $\mathrm{O}$ & 0.037062 & 0.962642 & 0.000000 \\
\hline $\mathrm{H}$ & -0.778299 & 1.536550 & 0.000000 \\
\hline
\end{tabular}
$\mathrm{H} \quad 0.000000 \quad 0.831851 \quad-1.549730$
$\mathrm{H} \quad 0.000000 \quad-0.831851 \quad-1.549730$

\begin{tabular}{cccc}
\multicolumn{5}{c}{$\left[{ }^{6} \mathrm{Fe}\left(\mathrm{H}_{2} \mathrm{O}\right)_{6}(\mathrm{H} 2 \mathrm{O}){ }_{12}\right]^{3+}$} \\
$\mathrm{E}=-1499.059213$ \\
$\mathrm{FE}$ & 0.000000 & 0.000000 & 0.000010 \\
$\mathrm{O}$ & 0.000000 & 0.000000 & -2.033097 \\
$\mathrm{O}$ & 0.000000 & -2.097981 & 0.000001 \\
$\mathrm{O}$ & 0.000000 & 0.000000 & 2.033124 \\
$\mathrm{O}$ & 0.000000 & 2.097981 & 0.000001 \\
$\mathrm{O}$ & 1.991451 & -0.000013 & -0.000056 \\
$\mathrm{O}$ & -1.991451 & 0.000013 & -0.000056 \\
$\mathrm{H}$ & 2.575271 & 0.285567 & 0.777033 \\
$\mathrm{H}$ & 2.575105 & -0.285611 & -0.777265 \\
$\mathrm{H}$ & 0.587504 & -2.670223 & 0.546449 \\
$\mathrm{H}$ & -0.587621 & -2.670165 & -0.546376 \\
$\mathrm{H}$ & 0.636527 & 0.509273 & -2.609797 \\
$\mathrm{H}$ & -0.636527 & -0.509273 & -2.609797 \\
$\mathrm{H}$ & -0.587504 & 2.670223 & 0.546449 \\
$\mathrm{H}$ & 0.587621 & 2.670165 & -0.546376 \\
$\mathrm{H}$ & 0.636557 & -0.509227 & 2.609818 \\
$\mathrm{H}$ & -0.636557 & 0.509227 & 2.609818 \\
$\mathrm{H}$ & -2.575271 & -0.285567 & 0.777033 \\
$\mathrm{H}$ & -2.575105 & 0.285611 & -0.777265 \\
$\mathrm{O}$ & -1.591566 & -3.701653 & -1.727845 \\
$\mathrm{H}$ & -2.426431 & -4.075880 & -1.396391 \\
$\mathrm{O}$ & 1.591819 & -3.701405 & 1.727878 \\
$\mathrm{H}$ & 2.426670 & -4.075550 & 1.396295 \\
$\mathrm{O}$ & -1.591819 & 3.701405 & 1.727878 \\
$\mathrm{H}$ & -1.124314 & 4.453482 & 2.131697 \\
$\mathrm{O}$ & 1.591566 & 3.701653 & -1.727845
\end{tabular}




$\begin{array}{rrrr}\mathrm{H} & 2.426431 & 4.075880 & -1.396391 \\ \mathrm{H} & -1.123935 & -4.453680 & -2.131611 \\ \mathrm{H} & 1.124314 & -4.453482 & 2.131697 \\ \mathrm{H} & -2.426670 & 4.075550 & 1.396295 \\ \mathrm{H} & 1.123935 & 4.453680 & -2.131611 \\ \mathrm{O} & 3.405053 & 0.578036 & 2.088678 \\ \mathrm{H} & 4.303129 & 0.924601 & 2.201050 \\ \mathrm{O} & 3.405328 & -0.577591 & -2.088760 \\ \mathrm{H} & 3.240648 & 0.076996 & -2.791992 \\ \mathrm{H} & 3.240922 & -0.076940 & 2.791673 \\ \mathrm{H} & 4.303745 & -0.923366 & -2.200850 \\ \mathrm{O} & -3.405053 & -0.578036 & 2.088678 \\ \mathrm{H} & -4.303129 & -0.924601 & 2.201050 \\ \mathrm{O} & -3.405328 & 0.577591 & -2.088760 \\ \mathrm{H} & -3.240648 & -0.076996 & -2.791992 \\ \mathrm{H} & -3.240922 & 0.076940 & 2.791673 \\ \mathrm{H} & -4.303745 & 0.923366 & -2.200850 \\ \mathrm{O} & -1.825172 & -1.402412 & -3.430622 \\ \mathrm{H} & -1.875632 & -2.301384 & -3.041272 \\ \mathrm{O} & 1.825172 & 1.402412 & -3.430622 \\ \mathrm{H} & 1.809563 & 1.514714 & -4.395690 \\ \mathrm{H} & -1.809563 & -1.514714 & -4.395690 \\ \mathrm{H} & 1.875632 & 2.301384 & -3.041272 \\ \mathrm{O} & 1.825308 & -1.402193 & 3.430715 \\ \mathrm{H} & 1.875804 & -2.301139 & 3.041304 \\ \mathrm{O} & -1.825308 & 1.402193 & 3.430715 \\ \mathrm{H} & -1.875804 & 2.301139 & 3.041304 \\ \mathrm{H} & 1.809715 & -1.514554 & 4.395777 \\ \mathrm{H} & -1.809715 & 1.514554 & 4.395777 \\ & & & \end{array}$

$\left[{ }^{6} \mathrm{Fe}\left(\mathrm{H}_{2} \mathrm{O}\right)_{5}(\mathrm{OH})\left(\mathrm{H}_{2} \mathrm{O}\right)_{12}\right]^{2+}$ (a)

$\mathrm{E}=-1498.856907$

$\begin{array}{llll}\mathrm{O} & 0.391611 & 1.378974 & -1.531759 \\ \mathrm{H} & 0.821157 & 2.258104 & -1.427541 \\ \mathrm{H} & -0.441798 & 1.487064 & -2.051766 \\ \mathrm{O} & -2.139057 & 1.433706 & -2.685136 \\ \mathrm{H} & -2.305626 & 1.702833 & -3.602069 \\ \mathrm{H} & -2.405971 & 0.484524 & -2.618286 \\ \mathrm{O} & 1.944417 & 3.673346 & -1.190115 \\ \mathrm{H} & 1.875760 & 4.488634 & -1.710884 \\ \mathrm{H} & 2.045516 & 3.933097 & -0.251899 \\ \mathrm{O} & -2.638491 & -1.338790 & -2.252102 \\ \mathrm{H} & -3.066508 & -1.892779 & -2.923131 \\ \mathrm{H} & -3.140449 & -1.485049 & -1.403727 \\ \mathrm{O} & -3.860468 & -1.740649 & 0.138953 \\ \mathrm{H} & -4.619155 & -2.267816 & 0.425514\end{array}$




$\begin{array}{lrrr}\mathrm{H} & -3.385878 & -1.445194 & 0.938334 \\ \mathrm{O} & 2.674607 & 3.139471 & 1.484528 \\ \mathrm{H} & 3.012440 & 3.616171 & 2.258863 \\ \mathrm{H} & 3.434457 & 2.674799 & 1.078561 \\ \mathrm{O} & 3.954403 & 1.794248 & -0.660885 \\ \mathrm{H} & 4.853568 & 1.698909 & -1.011462 \\ \mathrm{H} & 3.500178 & 2.466315 & -1.210453 \\ \mathrm{O} & -2.377097 & -0.836701 & 2.705119 \\ \mathrm{H} & -2.680133 & -1.049298 & 3.601564 \\ \mathrm{H} & -2.373638 & 0.139731 & 2.637095 \\ \mathrm{O} & -2.049972 & 2.207116 & 2.341338 \\ \mathrm{H} & -2.268657 & 2.841329 & 3.041982 \\ \mathrm{H} & -2.532971 & 2.498032 & 1.539230 \\ \mathrm{O} & -3.321359 & 2.433092 & -0.252062 \\ \mathrm{H} & -4.289804 & 2.476775 & -0.263762 \\ \mathrm{H} & -3.038725 & 2.411663 & -1.189551 \\ \mathrm{O} & -1.661620 & 0.179606 & 0.121374 \\ \mathrm{H} & -2.302680 & 0.903792 & -0.010769 \\ \mathrm{FE} & 0.131330 & -0.029196 & 0.038140 \\ \mathrm{O} & -0.047176 & -1.493238 & -1.423211 \\ \mathrm{H} & 0.526076 & -2.270513 & -1.610065 \\ \mathrm{H} & -0.963314 & -1.618739 & -1.780477 \\ \mathrm{O} & 1.598655 & -3.733724 & -1.545430 \\ \mathrm{H} & 1.722877 & -4.295693 & -2.326268 \\ \mathrm{H} & 2.489442 & -3.482895 & -1.227013 \\ \mathrm{O} & 0.457647 & 1.459634 & 1.472933 \\ \mathrm{H} & 1.251242 & 2.022800 & 1.619607 \\ \mathrm{H} & -0.350447 & 1.874662 & 1.858170 \\ \mathrm{O} & 2.229011 & -0.278214 & -0.025177 \\ \mathrm{H} & 2.877468 & 0.377783 & -0.371345 \\ \mathrm{H} & 2.710836 & -1.097184 & 0.237527 \\ \mathrm{O} & 3.384605 & -2.751146 & 0.433148 \\ \mathrm{H} & 4.305678 & -2.901202 & 0.697100 \\ \mathrm{H} & 2.821789 & -3.280078 & 1.036763 \\ \mathrm{O} & 1.024447 & -4.038244 & 1.202908 \\ \mathrm{H} & 0.794300 & -4.798524 & 1.759457 \\ \mathrm{H} & 0.952958 & -4.325373 & 0.270000 \\ \mathrm{O} & 0.089117 & -1.442956 & 1.594438 \\ \mathrm{H} & 0.296728 & -2.398847 & 1.485105 \\ \mathrm{H} & -0.767531 & -1.353003 & 2.083070\end{array}$

$\left[{ }^{6} \mathrm{Fe}\left(\mathrm{H}_{2} \mathrm{O}\right)_{5}(\mathrm{OH})\left(\mathrm{H}_{2} \mathrm{O}\right)_{12}\right]^{2+}(\mathrm{b})$

$\mathrm{E}=-1498.856909$

$\begin{array}{llll}\mathrm{O} & -0.086903 & 1.442630 & 1.595623\end{array}$

$\begin{array}{llll}\mathrm{H} & -0.291817 & 2.398953 & 1.485377\end{array}$

$\begin{array}{llll}\mathrm{H} & 0.769873 & 1.350906 & 2.083722\end{array}$ 


\begin{tabular}{|c|c|c|c|}
\hline $\mathrm{O}$ & 2.378287 & 0.831881 & 2.705428 \\
\hline $\mathrm{H}$ & 2.681366 & 1.044045 & 3.601967 \\
\hline $\mathrm{H}$ & 2.372934 & -0.144564 & 2.637636 \\
\hline $\mathrm{O}$ & -1.015636 & 4.041053 & 1.202998 \\
\hline $\mathrm{H}$ & -0.785033 & 4.800597 & 1.760371 \\
\hline $\mathrm{H}$ & -0.943465 & 4.329124 & 0.270475 \\
\hline $\mathrm{O}$ & -1.591980 & 3.737059 & -1.546162 \\
\hline $\mathrm{H}$ & -1.715350 & 4.298359 & -2.327611 \\
\hline $\mathrm{H}$ & -2.483133 & 3.488417 & -1.227020 \\
\hline $\mathrm{O}$ & 2.046298 & -2.211023 & 2.340754 \\
\hline $\mathrm{H}$ & 2.264543 & -2.845704 & 3.041116 \\
\hline $\mathrm{H}$ & 2.529131 & -2.501967 & 1.538581 \\
\hline $\mathrm{O}$ & 3.318177 & -2.436188 & -0.252694 \\
\hline $\mathrm{H}$ & 4.286686 & -2.478676 & -0.264028 \\
\hline $\mathrm{H}$ & 3.035784 & -2.416953 & -1.190236 \\
\hline $\mathrm{O}$ & 2.135283 & -1.436703 & -2.686575 \\
\hline $\mathrm{H}$ & 2.300694 & -1.705606 & -3.603779 \\
\hline $\mathrm{H}$ & 2.403664 & -0.487969 & -2.619518 \\
\hline $\mathrm{O}$ & 2.640131 & 1.334719 & -2.251887 \\
\hline $\mathrm{H}$ & 3.069646 & 1.888079 & -2.922482 \\
\hline $\mathrm{H}$ & 3.142930 & 1.478508 & -1.403629 \\
\hline $\mathrm{O}$ & 3.867191 & 1.727780 & 0.138630 \\
\hline $\mathrm{H}$ & 4.624967 & 2.256178 & 0.425384 \\
\hline $\mathrm{H}$ & 3.389946 & 1.436170 & 0.937855 \\
\hline $\mathrm{O}$ & -3.378282 & 2.758869 & 0.433954 \\
\hline $\mathrm{H}$ & -4.298458 & 2.910978 & 0.699855 \\
\hline $\mathrm{H}$ & -2.812918 & 3.286763 & 1.036160 \\
\hline $\mathrm{O}$ & 1.660664 & -0.182516 & 0.121292 \\
\hline $\mathrm{H}$ & 2.301395 & -0.906834 & -0.012173 \\
\hline FE & -0.131782 & 0.029365 & 0.038110 \\
\hline $\mathrm{O}$ & 0.049783 & 1.494477 & -1.421544 \\
\hline $\mathrm{H}$ & -0.523255 & 2.271481 & -1.610386 \\
\hline $\mathrm{H}$ & 0.965902 & 1.618577 & -1.779401 \\
\hline $\mathrm{O}$ & -0.460434 & -1.458901 & 1.473202 \\
\hline $\mathrm{H}$ & 0.347384 & -1.875183 & 1.857653 \\
\hline $\mathrm{H}$ & -1.254289 & -2.021821 & 1.618993 \\
\hline $\mathrm{O}$ & -2.679026 & -3.138448 & 1.483207 \\
\hline $\mathrm{H}$ & -3.015314 & -3.615265 & 2.258137 \\
\hline $\mathrm{H}$ & -3.439219 & -2.672165 & 1.079756 \\
\hline $\mathrm{O}$ & -3.959217 & -1.787252 & -0.657841 \\
\hline $\mathrm{H}$ & -4.859143 & -1.689604 & -1.005859 \\
\hline $\mathrm{H}$ & -3.507884 & -2.459574 & -1.209431 \\
\hline $\mathrm{O}$ & -1.952889 & -3.668587 & -1.192590 \\
\hline $\mathrm{H}$ & -1.885984 & -4.483384 & -1.714341 \\
\hline $\mathrm{H}$ & -2.053857 & -3.929255 & -0.254579 \\
\hline $\mathrm{O}$ & -2.228867 & 0.282718 & -0.025040 \\
\hline
\end{tabular}




$\begin{array}{rrrc}\mathrm{H} & -2.878926 & -0.372356 & -0.369872 \\ \mathrm{H} & -2.708828 & 1.102616 & 0.237946 \\ \mathrm{O} & -0.394519 & -1.378125 & -1.531638 \\ \mathrm{H} & -0.826613 & -2.256162 & -1.428441 \\ \mathrm{H} & 0.438473 & -1.487755 & -2.052002\end{array}$

$\left[{ }^{6} \mathrm{Fe}\left(\mathrm{H}_{2} \mathrm{O}\right)_{5}(\mathrm{OH})\left(\mathrm{H}_{2} \mathrm{O}\right)_{12}\right]^{2+}(\mathrm{c})$

$\mathrm{E}=-1498.854451$

$\begin{array}{cccc}\mathrm{O} & -2.072825 & 0.184314 & 0.785189 \\ \mathrm{H} & -2.321627 & -0.461419 & 1.490255 \\ \mathrm{H} & -2.868196 & 0.410771 & 0.247746 \\ \mathrm{O} & -4.171274 & 0.506214 & -1.008090 \\ \mathrm{H} & -5.043128 & 0.894961 & -0.838228 \\ \mathrm{H} & -3.806414 & 0.948410 & -1.806161 \\ \mathrm{O} & -2.422214 & -1.447225 & 2.961331 \\ \mathrm{H} & -3.270168 & -1.655617 & 3.382865 \\ \mathrm{H} & -1.952416 & -2.301126 & 2.821119 \\ \mathrm{O} & -0.809567 & -3.603671 & 2.239548 \\ \mathrm{H} & -0.175793 & -3.953046 & 2.886841 \\ \mathrm{H} & -1.181134 & -4.379968 & 1.790796 \\ \mathrm{O} & -2.432245 & 1.366459 & -3.036714 \\ \mathrm{H} & -2.563939 & 1.677261 & -3.945666 \\ \mathrm{H} & -1.880366 & 0.557661 & -3.069502 \\ \mathrm{O} & -0.810774 & 2.017969 & -0.927452 \\ \mathrm{H} & -0.614869 & 2.910309 & -0.569606 \\ \mathrm{H} & -1.342916 & 2.073139 & -1.749478 \\ \mathrm{O} & 0.319877 & 4.075707 & 0.478841 \\ \mathrm{H} & 0.037685 & 4.943991 & 0.805391 \\ \mathrm{H} & 0.294392 & 3.447360 & 1.229737 \\ \mathrm{O} & -0.910684 & -0.688730 & -1.846401 \\ \mathrm{H} & -1.658602 & -1.324438 & -1.668872 \\ \mathrm{H} & -0.188163 & -1.229129 & -2.290257 \\ \mathrm{O} & 1.000175 & -2.177336 & -2.898454 \\ \mathrm{H} & 1.080246 & -2.557205 & -3.784232 \\ \mathrm{H} & 1.905272 & -2.050161 & -2.541380 \\ \mathrm{O} & -3.150156 & -2.110997 & -1.365896 \\ \mathrm{H} & -3.528942 & -2.837386 & -1.881996 \\ \mathrm{H} & -3.831868 & -1.413808 & -1.298883 \\ \mathrm{O} & 3.405329 & -1.410988 & -1.633136 \\ \mathrm{H} & 4.122792 & -1.125206 & -2.219700 \\ \mathrm{H} & 3.825442 & -2.047146 & -0.997927 \\ \mathrm{O} & 4.675369 & -3.191777 & -0.013936 \\ \mathrm{H} & 4.963061 & -4.065368 & -0.318038 \\ \mathrm{H} & 5.230466 & -2.973546 & 0.748915 \\ \mathrm{O} & 0.461659 & 1.483638 & 1.594085 \\ \mathrm{H} & 1.447173 & 1.451302 & 1.756464\end{array}$




$\begin{array}{cccc}\mathrm{H} & 0.027401 & 1.198318 & 2.451653 \\ \mathrm{O} & -0.652234 & 0.616059 & 3.841743 \\ \mathrm{H} & -0.752714 & 1.028771 & 4.710665 \\ \mathrm{H} & -1.311903 & -0.101831 & 3.768668 \\ \mathrm{O} & 3.124682 & 1.703903 & 1.775273 \\ \mathrm{H} & 3.703543 & 1.866394 & 2.533509 \\ \mathrm{H} & 3.374330 & 2.327002 & 1.067106 \\ \mathrm{O} & 2.711501 & 3.114265 & -0.647662 \\ \mathrm{H} & 3.255978 & 3.619148 & -1.270902 \\ \mathrm{H} & 1.975538 & 3.700856 & -0.365956 \\ \mathrm{O} & 1.640742 & 0.570781 & -0.985213 \\ \mathrm{H} & 2.049236 & 1.468432 & -1.009423 \\ \mathrm{H} & 2.346855 & -0.120818 & -1.060779 \\ \mathrm{O} & 0.390787 & -1.348626 & 0.732701 \\ \mathrm{H} & 0.012183 & -2.169746 & 1.092717 \\ \mathrm{FE} & -0.143985 & 0.190893 & -0.017828\end{array}$

\begin{tabular}{cccc}
\multicolumn{5}{c}{$\left.\mathrm{Fe}\left(\mathrm{H}_{2} \mathrm{O}\right)_{5}(\mathrm{OH})\left(\mathrm{H}_{2} \mathrm{O}\right)_{12}\right]^{2+}(\mathrm{d})$} \\
$\mathrm{E}=-1498.859647$ \\
$\mathrm{O}$ & -1.454198 & 1.366971 & -0.281415 \\
$\mathrm{H}$ & -2.363633 & 1.289646 & -0.638116 \\
$\mathrm{O}$ & -4.222291 & 0.819531 & -0.900295 \\
$\mathrm{H}$ & -4.855967 & 1.412818 & -1.332401 \\
$\mathrm{H}$ & -4.289106 & -0.046441 & -1.349626 \\
$\mathrm{O}$ & 1.353275 & 4.544989 & 0.408598 \\
$\mathrm{H}$ & 1.580122 & 5.471846 & 0.569141 \\
$\mathrm{H}$ & 0.584579 & 4.318684 & 0.969140 \\
$\mathrm{O}$ & -3.776324 & -1.877583 & -1.895331 \\
$\mathrm{H}$ & -4.038036 & -2.545418 & -1.239647 \\
$\mathrm{H}$ & -4.147824 & -2.185360 & -2.738783 \\
$\mathrm{O}$ & -0.711223 & 3.109627 & 1.675486 \\
$\mathrm{H}$ & -1.346945 & 3.360409 & 2.363062 \\
$\mathrm{H}$ & -1.221370 & 2.685260 & 0.941662 \\
$\mathrm{O}$ & -1.682515 & -1.123357 & 1.104885 \\
$\mathrm{H}$ & -1.346033 & -1.732580 & 1.822104 \\
$\mathrm{H}$ & -2.435289 & -0.584465 & 1.480959 \\
$\mathrm{O}$ & -3.744749 & 0.392805 & 1.845099 \\
$\mathrm{H}$ & -4.422732 & 0.251902 & 2.521439 \\
$\mathrm{H}$ & -4.197472 & 0.629254 & 1.010522 \\
$\mathrm{O}$ & -0.704879 & -2.660438 & 3.040010 \\
$\mathrm{H}$ & -1.141752 & -3.067498 & 3.801124 \\
$\mathrm{H}$ & 0.243460 & -2.568436 & 3.248807 \\
$\mathrm{O}$ & 2.104573 & -2.343191 & 2.666235 \\
$\mathrm{H}$ & 2.662897 & -3.100334 & 2.901684 \\
$\mathrm{H}$ & 2.660947 & -1.538663 & 2.741543 \\
$\mathrm{O}$ & 3.239002 & 0.152157 & 2.027185
\end{tabular}




\begin{tabular}{|c|c|c|c|}
\hline $\mathrm{H}$ & & 0.711400 & \\
\hline $\mathrm{H}$ & & 0.040299 & \\
\hline $\mathrm{O}$ & 1.023554 & -1.703167 & 0.238884 \\
\hline $\mathrm{H}$ & & -2.050972 & 1.094775 \\
\hline & 1.482406 & -2.170876 & -0.493278 \\
\hline $\mathrm{C}$ & & -2.490149 & 0058 \\
\hline & 3.024626 & -3.365646 & 3131 \\
\hline $\mathrm{H}$ & & -2.199831 & 3013 \\
\hline $\mathrm{O}$ & & -1.081746 & 7810 \\
\hline $\mathrm{H}$ & 1.13 & -0.2 & 316 \\
\hline $\mathrm{H}$ & & -0.9 & \\
\hline $\mathrm{O}$ & 2 & 8690 & -1.3 \\
\hline $\mathrm{H}$ & & & \\
\hline $\mathrm{H}$ & 1.11 & 1.6 & -1.5 \\
\hline $\mathrm{O}$ & & & \\
\hline $\mathrm{H}$ & 0.9 & & -2.5 \\
\hline $\mathrm{H}$ & & & \\
\hline $\mathrm{O}$ & & -0.1 & -0 . \\
\hline $\mathrm{H}$ & & & -1.1 \\
\hline $\mathrm{H}$ & & & \\
\hline $\mathrm{O}$ & & -1. & -1 . \\
\hline $\mathrm{H}$ & & & -2 . \\
\hline $\mathrm{H}$ & 7 & -1.5 & -1 . \\
\hline $\mathrm{O}$ & & & \\
\hline $\mathrm{H}$ & & & \\
\hline $\mathrm{H}$ & & & \\
\hline $\mathrm{FE}$ & 47 & $-0 .($ & -0 . \\
\hline \multicolumn{4}{|c|}{$\left[{ }^{6} \mathrm{Fe}\left(\mathrm{H}_{2} \mathrm{O}\right)_{5}(\mathrm{OH})\left(\mathrm{H}_{2} \mathrm{O}\right)_{12}\right]^{2+}(\mathrm{e})$} \\
\hline \multicolumn{4}{|c|}{$E=-1498.854451$} \\
\hline $\mathrm{O}$ & -2.073077 & & \\
\hline & -2.3 & -0. & \\
\hline & $28 t$ & & 13 \\
\hline & & & \\
\hline & & & \\
\hline & -3.8 & 0.9 & -1.8 \\
\hline $\mathrm{O}$ & -2.4 & -1.4 & \\
\hline $\mathrm{H}$ & -3.27 & -1.6 & 3377 \\
\hline & -1.9 & -2.2 & \\
\hline $\mathrm{O}$ & -0.80 & -3.601883 & 2.242256 \\
\hline & & -3.9 & \\
\hline $\mathrm{H}$ & -1.178210 & -4.378386 & 1.793049 \\
\hline & -2.43 & 1.363058 & -3.038099 \\
\hline $\mathrm{H}$ & -2.563790 & 1.673213 & -3.947289 \\
\hline $\mathrm{H}$ & -1.879926 & 0.554518 & -3.070163 \\
\hline $\mathrm{O}$ & -3.149102 & -2.113356 & -1.365502 \\
\hline
\end{tabular}




$\begin{array}{lrrr}\mathrm{H} & -3.527660 & -2.840156 & -1.881185 \\ \mathrm{H} & -3.830966 & -1.416265 & -1.299033 \\ \mathrm{O} & -0.909913 & -0.690440 & -1.846280 \\ \mathrm{H} & -1.657454 & -1.326461 & -1.668344 \\ \mathrm{H} & -0.186947 & -1.230872 & -2.289498 \\ \mathrm{O} & 1.001842 & -2.178797 & -2.896447 \\ \mathrm{H} & 1.082280 & -2.559041 & -3.782039 \\ \mathrm{H} & 1.906822 & -2.050224 & -2.539539 \\ \mathrm{O} & 3.406360 & -1.410311 & -1.631322 \\ \mathrm{H} & 4.123505 & -1.124616 & -2.218328 \\ \mathrm{H} & 3.826944 & -2.046139 & -0.996079 \\ \mathrm{O} & 4.678031 & -3.189870 & -0.012234 \\ \mathrm{H} & 5.232828 & -2.971493 & 0.750790 \\ \mathrm{H} & 4.965877 & -4.063492 & -0.316095 \\ \mathrm{O} & 1.640830 & 0.570850 & -0.985268 \\ \mathrm{H} & 2.048657 & 1.468775 & -1.009765 \\ \mathrm{H} & 2.347638 & -0.120188 & -1.059814 \\ \mathrm{O} & 2.710069 & 3.115533 & -0.648754 \\ \mathrm{H} & 3.254293 & 3.620406 & -1.272225 \\ \mathrm{H} & 1.973608 & 3.701784 & -0.367688 \\ \mathrm{O} & 0.317133 & 4.076045 & 0.476422 \\ \mathrm{H} & 0.034561 & 4.944360 & 0.802559 \\ \mathrm{H} & 0.292129 & 3.448092 & 1.227670 \\ \mathrm{O} & -0.811529 & 2.016796 & -0.928929 \\ \mathrm{H} & -0.616278 & 2.909469 & -0.571518 \\ \mathrm{H} & -1.343470 & 2.071195 & -1.751139 \\ \mathrm{O} & 0.460351 & 1.484941 & 1.593275 \\ \mathrm{H} & 1.445813 & 1.453008 & 1.755948 \\ \mathrm{H} & 0.025909 & 1.200059 & 2.450872 \\ \mathrm{O} & 3.123303 & 1.706375 & 1.774875 \\ \mathrm{H} & 3.701810 & 1.869351 & 2.533278 \\ \mathrm{H} & 3.373002 & 2.329318 & 1.066596 \\ \mathrm{O} & -0.654106 & 0.618842 & 3.841326 \\ \mathrm{H} & -0.754476 & 1.031901 & 4.710093 \\ \mathrm{H} & -1.313244 & -0.099597 & 3.768895 \\ \mathrm{O} & 0.391629 & -1.348109 & 0.733355 \\ \mathrm{H} & 0.013595 & -2.168466 & 1.095750 \\ \mathrm{FE} & -0.144023 & 0.190607 & -0.018135\end{array}$

$\left[{ }^{6} \mathrm{Fe}\left(\mathrm{H}_{2} \mathrm{O}\right)_{5}(\mathrm{OH})\left(\mathrm{H}_{2} \mathrm{O}\right)_{12}\right]^{2+}$ (f)

$\mathrm{E}=-1498.859647$

$\begin{array}{lrrr}\mathrm{O} & 1.022514 & -1.703249 & 0.241358 \\ \mathrm{H} & 1.482915 & -2.171482 & -0.489527 \\ \mathrm{H} & 1.374869 & -2.048922 & 1.098187 \\ \mathrm{O} & 2.656047 & -2.493584 & -1.883904 \\ \mathrm{H} & 3.026699 & -3.369499 & -2.075157\end{array}$




\begin{tabular}{|c|c|c|c|}
\hline $\mathrm{H}$ & .169005 & -2.204802 & \\
\hline & 2.101900 & -2.338428 & \\
\hline & 2.660215 & -3.095385 & \\
\hline & 2.658393 & -1.533852 & 2.744543 \\
\hline & 0.947622 & -1.088879 & \\
\hline & 1.138722 & -0.217341 & \\
\hline & & -0.9 & \\
\hline & 3.238191 & & \\
\hline & & & \\
\hline & 3.61 & 0.0 & \\
\hline & & & \\
\hline & 1.12 & & -1.5 \\
\hline & 2.0 & & \\
\hline & 3.768 & -0.1 & -0. \\
\hline & 4.538 & 0.2 & -1.1 \\
\hline & & & \\
\hline & 1.33 & 3.1 & -1.8 \\
\hline & & & -2.5 \\
\hline & & 3.7 & $-1 .($ \\
\hline & & & \\
\hline & & & \\
\hline & & & \\
\hline C & -0.7 & & \\
\hline & & & \\
\hline $\mathrm{H}$ & & & \\
\hline $\mathrm{O}$ & -1.4 & & -0.2 \\
\hline $\mathrm{H}$ & -2.36 & & \\
\hline $\mathrm{O}$ & -4.22 & 0.8 & -0 . \\
\hline $\mathrm{H}$ & -4.8 & 1.4 & \\
\hline $\mathrm{H}$ & $-4.2 \xi$ & -0.0 & \\
\hline $\mathrm{O}$ & -3.77 & -1.8 & \\
\hline $\mathrm{H}$ & -4.03 & -2.5 & -1.2 \\
\hline $\mathrm{H}$ & & -2.1 & -2. \\
\hline $\mathrm{O}$ & -1.68 & -1.1 & \\
\hline $\mathrm{H}$ & -2.43 & -0.5 & \\
\hline $\mathrm{H}$ & -1.347524 & -1.72 & 1.8 \\
\hline $\mathrm{O}$ & -0.707571 & -2.6 & \\
\hline $\mathrm{H}$ & -1.145004 & -3.060 & 3.8 \\
\hline $\mathrm{H}$ & 0.240539 & -2.563325 & 3.2 \\
\hline $\mathrm{O}$ & -3.745607 & & \\
\hline $\mathrm{H}$ & -4.424073 & 0.256730 & 2.5 \\
\hline $\mathrm{H}$ & -4.197764 & 0.631536 & \\
\hline $\mathrm{O}$ & -1.035931 & -1.231255 & -1.702895 \\
\hline $\mathrm{H}$ & & & -1.807044 \\
\hline $\mathrm{H}$ & -0.553030 & -1.282235 & -2.556057 \\
\hline $\mathrm{O}$ & 0.589457 & 0.764950 & 1.572520 \\
\hline
\end{tabular}




\begin{tabular}{cccc}
$\mathrm{H}$ & 0.241879 & 1.660764 & 1.824298 \\
$\mathrm{H}$ & 1.551333 & 0.687973 & 1.780812 \\
$\mathrm{FE}$ & -0.352917 & -0.093117 & -0.065408 \\
\multicolumn{5}{c}{} \\
{$\left[\begin{array}{l}6 \\
{[}\end{array} \mathrm{Fe}\left(\mathrm{H}_{2} \mathrm{O}\right)_{6}\right]^{3+}$} \\
$\mathrm{E}=--581.0284859$ \\
$\mathrm{FE}$ & -0.000001 & 0.000012 & 0.000000 \\
$\mathrm{O}$ & -2.097364 & 0.006281 & 0.000450 \\
$\mathrm{O}$ & 0.006685 & 2.015885 & 0.004502 \\
$\mathrm{O}$ & 2.097369 & -0.006367 & -0.000465 \\
$\mathrm{O}$ & -0.006651 & -2.015867 & -0.004491 \\
$\mathrm{O}$ & -0.000491 & 0.004887 & -1.917370 \\
$\mathrm{O}$ & 0.000442 & -0.004854 & 1.917369 \\
$\mathrm{H}$ & -0.003284 & -0.796257 & -2.486114 \\
$\mathrm{H}$ & 0.001382 & 0.809205 & -2.481619 \\
$\mathrm{H}$ & 0.805155 & 2.584324 & 0.004618 \\
$\mathrm{H}$ & -0.787792 & 2.589880 & 0.008776 \\
$\mathrm{H}$ & -2.685236 & 0.010877 & -0.783695 \\
$\mathrm{H}$ & -2.685021 & 0.006536 & 0.784773 \\
$\mathrm{H}$ & 0.787845 & -2.589844 & -0.006798 \\
$\mathrm{H}$ & -0.805108 & -2.584320 & -0.006514 \\
$\mathrm{H}$ & 2.685014 & -0.005943 & -0.784797 \\
$\mathrm{H}$ & 2.685255 & -0.011618 & 0.783664 \\
$\mathrm{H}$ & 0.005069 & 0.796288 & 2.486105 \\
$\mathrm{H}$ & -0.003177 & -0.809161 & 2.481627
\end{tabular}

\begin{tabular}{cccc}
\multicolumn{5}{c}{$\left.\mathrm{C} F\left(\mathrm{H}_{2} \mathrm{O}\right)_{5}(\mathrm{OH})\right]^{2+}(\mathrm{a})$} \\
$\mathrm{E}=-581.0301229$ \\
$\mathrm{O}$ & 2.024108 & 0.574270 & -0.019207 \\
$\mathrm{H}$ & 2.559515 & 0.721249 & 0.780115 \\
$\mathrm{H}$ & 2.587691 & 0.736309 & -0.797036 \\
$\mathrm{O}$ & 0.581900 & -2.038602 & 0.181608 \\
$\mathrm{H}$ & -0.024501 & -2.772960 & -0.023262 \\
$\mathrm{H}$ & 1.473749 & -2.347580 & -0.059417 \\
$\mathrm{O}$ & -0.003473 & 0.003396 & 1.972833 \\
$\mathrm{H}$ & -0.223424 & 0.762460 & 2.540645 \\
$\mathrm{H}$ & 0.215526 & -0.752283 & 2.545506 \\
$\mathrm{O}$ & -0.582406 & 2.039194 & 0.174399 \\
$\mathrm{H}$ & 0.023442 & 2.773020 & -0.034064 \\
$\mathrm{H}$ & -1.474584 & 2.346879 & -0.067103 \\
$\mathrm{O}$ & -2.023819 & -0.574885 & -0.023569 \\
$\mathrm{H}$ & -2.585145 & -0.739162 & -0.802568 \\
$\mathrm{H}$ & -2.561611 & -0.719213 & 0.774631 \\
$\mathrm{FE}$ & 0.000318 & -0.000357 & -0.182505 \\
$\mathrm{O}$ & 0.003223 & -0.002791 & -1.936719 \\
$\mathrm{H}$ & 0.004819 & -0.004099 & -2.907071
\end{tabular}




$$
\begin{array}{cccc}
\multicolumn{5}{c}{\left.\mathrm{Fe}\left(\mathrm{H}_{2} \mathrm{O}\right)_{5}(\mathrm{OH})\right]^{2+}(\mathrm{b})} \\
\mathrm{E}=-581.0301241 \\
\mathrm{O} & 2.023165 & -0.578193 & -0.021713 \\
\mathrm{H} & 2.585130 & -0.741337 & -0.800479 \\
\mathrm{H} & 2.558700 & -0.729311 & 0.776743 \\
\mathrm{O} & 0.585761 & 2.038033 & 0.174776 \\
\mathrm{H} & 1.482672 & 2.341252 & -0.054471 \\
\mathrm{H} & -0.014171 & 2.773162 & -0.045850 \\
\mathrm{O} & 0.001224 & 0.003438 & 1.972996 \\
\mathrm{H} & -0.215560 & -0.752884 & 2.545650 \\
\mathrm{H} & 0.218498 & 0.763251 & 2.540829 \\
\mathrm{O} & -2.023159 & 0.578345 & -0.021294 \\
\mathrm{H} & -2.557923 & 0.731391 & 0.777310 \\
\mathrm{H} & -2.585609 & 0.740570 & -0.799900 \\
\mathrm{O} & -0.585527 & -2.037525 & 0.181384 \\
\mathrm{H} & -1.482369 & -2.341941 & -0.046543 \\
\mathrm{H} & 0.014514 & -2.773474 & -0.036186 \\
\mathrm{FE} & -0.000148 & -0.000412 & -0.182512 \\
\mathrm{O} & -0.001241 & -0.003456 & -1.936734 \\
\mathrm{H} & -0.001836 & -0.005106 & -2.907092
\end{array}
$$

$$
\begin{array}{lccc}
\multicolumn{5}{c}{\left.\mathrm{C} \mathrm{Fe}\left(\mathrm{H}_{2} \mathrm{O}\right)_{5}(\mathrm{OH})\right]^{2+}(\mathrm{c})} \\
\mathrm{E}=-581.0301458 \\
\mathrm{O} & 0.671991 & -2.010929 & 0.176033 \\
\mathrm{H} & 1.582979 & -2.274163 & -0.047099 \\
\mathrm{H} & 0.106569 & -2.771376 & -0.049866 \\
\mathrm{O} & 1.997099 & 0.662699 & -0.020868 \\
\mathrm{H} & 2.523345 & 0.842051 & 0.777924 \\
\mathrm{H} & 2.552105 & 0.849499 & -0.799298 \\
\mathrm{O} & 0.000044 & -0.001407 & 1.973387 \\
\mathrm{H} & 0.249590 & -0.750383 & 2.542399 \\
\mathrm{H} & -0.248664 & 0.745831 & 2.544968 \\
\mathrm{O} & -1.996743 & -0.663861 & -0.021574 \\
\mathrm{H} & -2.551419 & -0.850461 & -0.800286 \\
\mathrm{H} & -2.523054 & -0.844163 & 0.776962 \\
\mathrm{O} & -0.671882 & 2.010992 & 0.179133 \\
\mathrm{H} & -1.583294 & 2.274192 & -0.042171 \\
\mathrm{H} & -0.106899 & 2.771573 & -0.047297 \\
\mathrm{FE} & -0.000096 & 0.000325 & -0.182449 \\
\mathrm{O} & -0.000304 & 0.002017 & -1.936789 \\
\mathrm{H} & -0.000391 & 0.002866 & -2.907148
\end{array}
$$

$\left[{ }^{6} \mathrm{Fe}\left(\mathrm{H}_{2} \mathrm{O}\right)_{5}(\mathrm{OH})\right]^{2+}(\mathrm{d})$

$\mathrm{E}=-581.0301441$

$\begin{array}{llll}\text { O } & -0.000965 & 0.004475 & 1.972926\end{array}$ 


$\begin{array}{cccc}\mathrm{H} & 0.242552 & 0.756633 & 2.540262 \\ \mathrm{H} & -0.242548 & -0.743980 & 2.545945 \\ \mathrm{O} & 0.654169 & 2.017122 & 0.174027 \\ \mathrm{H} & 0.080338 & 2.771686 & -0.050286 \\ \mathrm{H} & 1.562006 & 2.289784 & -0.050524 \\ \mathrm{O} & 2.002333 & -0.645551 & -0.018232 \\ \mathrm{H} & 2.560740 & -0.828874 & -0.795080 \\ \mathrm{H} & 2.529098 & -0.817075 & 0.781930 \\ \mathrm{O} & -2.001814 & 0.647022 & -0.022361 \\ \mathrm{H} & -2.529357 & 0.821283 & 0.776699 \\ \mathrm{H} & -2.559757 & 0.826683 & -0.800403 \\ \mathrm{O} & -0.654307 & -2.016848 & 0.181100 \\ \mathrm{H} & -0.082074 & -2.773017 & -0.041913 \\ \mathrm{H} & -1.563377 & -2.290424 & -0.037262 \\ \mathrm{FE} & -0.000040 & -0.000730 & -0.183198 \\ \mathrm{O} & 0.000832 & -0.004629 & -1.937285 \\ \mathrm{H} & 0.001429 & -0.006442 & -2.907636\end{array}$

$\begin{array}{cccc}{\left[{ }^{6} \mathrm{Fe}\left(\mathrm{H}_{2} \mathrm{O}\right)_{5}(\mathrm{OH})\right]^{2+}(\mathrm{e})} \\ \mathrm{E}=-581.0301305 \\ \mathrm{O} & -2.017319 & -0.598537 & -0.021149 \\ \mathrm{H} & -2.547652 & -0.766059 & 0.777484 \\ \mathrm{H} & -2.578194 & -0.766181 & -0.799709 \\ \mathrm{O} & -0.000895 & -0.002765 & 1.973493 \\ \mathrm{H} & -0.225208 & 0.751537 & 2.545858 \\ \mathrm{H} & 0.223617 & -0.760241 & 2.541576 \\ \mathrm{O} & 0.605592 & -2.032421 & 0.174624 \\ \mathrm{H} & 1.510088 & -2.323720 & -0.039814 \\ \mathrm{H} & 0.017985 & -2.772557 & -0.061488 \\ \mathrm{O} & 2.017610 & 0.597611 & -0.018270 \\ \mathrm{H} & 2.579572 & 0.765589 & -0.795970 \\ \mathrm{H} & 2.547559 & 0.762401 & 0.781188 \\ \mathrm{O} & -0.605512 & 2.032275 & 0.179489 \\ \mathrm{H} & -1.510083 & 2.324569 & -0.033396 \\ \mathrm{H} & -0.018096 & 2.773031 & -0.055260 \\ \mathrm{FE} & -0.000044 & 0.000453 & -0.183111 \\ \mathrm{O} & 0.000591 & 0.003252 & -1.937192 \\ \mathrm{H} & 0.001027 & 0.004520 & -2.907548\end{array}$

$\left[{ }^{6} \mathrm{Fe}\left(\mathrm{H}_{2} \mathrm{O}\right)_{5}(\mathrm{OH})\right]^{2+}(\mathrm{f})$

$\mathrm{E}=-581.0301411$

$\begin{array}{rrrr}\mathrm{O} & -0.638253 & 2.022146 & 0.178532 \\ \mathrm{H} & -0.061689 & 2.773056 & -0.050963 \\ \mathrm{H} & -1.546086 & 2.300538 & -0.038706 \\ \mathrm{O} & 2.007207 & 0.629296 & -0.017271 \\ \mathrm{H} & 2.567321 & 0.809025 & -0.793710\end{array}$




$\begin{array}{lccc}\mathrm{H} & 2.533906 & 0.798548 & 0.783407 \\ \mathrm{O} & -0.001050 & -0.002627 & 1.973432 \\ \mathrm{H} & 0.234920 & -0.756795 & 2.541303 \\ \mathrm{H} & -0.236756 & 0.747988 & 2.546084 \\ \mathrm{O} & 0.638282 & -2.021760 & 0.174772 \\ \mathrm{H} & 0.060704 & -2.771395 & -0.056377 \\ \mathrm{H} & 1.545396 & -2.298931 & -0.047044 \\ \mathrm{O} & -2.006546 & -0.631574 & -0.020490 \\ \mathrm{H} & -2.533857 & -0.804029 & 0.779096 \\ \mathrm{H} & -2.566422 & -0.807134 & -0.798063 \\ \mathrm{FE} & -0.000120 & 0.000780 & -0.183455 \\ \mathrm{O} & 0.000771 & 0.003034 & -1.937406 \\ \mathrm{H} & 0.002391 & 0.000737 & -2.907741\end{array}$

$\left[{ }^{1} \mathrm{Sc}\left(\mathrm{H}_{2} \mathrm{O}\right)_{6}\left(\mathrm{H}_{2} \mathrm{O}\right)_{12}\right]^{3+}$

$\mathrm{E}=-2136.480403$

$\begin{array}{lrrc}\mathrm{SC} & 0.000000 & 0.000000 & 0.000008 \\ \mathrm{O} & 0.000000 & 0.000000 & -2.135977 \\ \mathrm{O} & 2.190170 & 0.000007 & 0.000041 \\ \mathrm{O} & 0.000000 & 0.000000 & 2.135998 \\ \mathrm{O} & -2.190170 & -0.000007 & 0.000041 \\ \mathrm{O} & 0.000000 & 2.103202 & -0.000010 \\ \mathrm{O} & 0.000000 & -2.103202 & -0.000010 \\ \mathrm{H} & -0.238137 & 2.694557 & 0.784305 \\ \mathrm{H} & 0.238142 & 2.694549 & -0.784332 \\ \mathrm{H} & 2.769770 & 0.550299 & 0.578801 \\ \mathrm{H} & 2.769779 & -0.550292 & -0.578705 \\ \mathrm{H} & -0.504475 & 0.633494 & -2.719937 \\ \mathrm{H} & 0.504475 & -0.633494 & -2.719937 \\ \mathrm{H} & -2.769770 & -0.550299 & 0.578801 \\ \mathrm{H} & -2.769779 & 0.550292 & -0.578705 \\ \mathrm{H} & 0.504496 & 0.633480 & 2.719954 \\ \mathrm{H} & -0.504496 & -0.633480 & 2.719954 \\ \mathrm{H} & 0.238137 & -2.694557 & 0.784305 \\ \mathrm{H} & -0.238142 & -2.694549 & -0.784332 \\ \mathrm{O} & 3.753324 & -1.519192 & -1.813194 \\ \mathrm{H} & 4.147489 & -2.350976 & -1.497130 \\ \mathrm{O} & 3.753282 & 1.519452 & 1.813153 \\ \mathrm{H} & 4.147278 & 2.351292 & 1.497023 \\ \mathrm{O} & -3.753282 & -1.519452 & 1.813153 \\ \mathrm{H} & -4.489104 & -1.036964 & 2.229256 \\ \mathrm{O} & -3.753324 & 1.519192 & -1.813194 \\ \mathrm{H} & -4.147489 & 2.350976 & -1.497130 \\ \mathrm{H} & 4.489026 & -1.036559 & -2.229342 \\ \mathrm{H} & 4.489104 & 1.036964 & 2.229256 \\ \mathrm{H} & -4.147278 & -2.351292 & 1.497023\end{array}$




$\begin{array}{crrc}\mathrm{H} & -4.489026 & 1.036559 & -2.229342 \\ \mathrm{O} & -0.473526 & 3.500896 & 2.139219 \\ \mathrm{H} & -0.794754 & 4.404039 & 2.281633 \\ \mathrm{O} & 0.473449 & 3.500870 & -2.139276 \\ \mathrm{H} & -0.173638 & 3.294150 & -2.837936 \\ \mathrm{H} & 0.173504 & 3.294175 & 2.837929 \\ \mathrm{H} & 0.794702 & 4.403994 & -2.281744 \\ \mathrm{O} & 0.473526 & -3.500896 & 2.139219 \\ \mathrm{H} & 0.794754 & -4.404039 & 2.281633 \\ \mathrm{O} & -0.473449 & -3.500870 & -2.139276 \\ \mathrm{H} & 0.173638 & -3.294150 & -2.837936 \\ \mathrm{H} & -0.173504 & -3.294175 & 2.837929 \\ \mathrm{H} & -0.794702 & -4.403994 & -2.281744 \\ \mathrm{O} & 1.434204 & -1.828396 & -3.508368 \\ \mathrm{H} & 2.335878 & -1.842997 & -3.123265 \\ \mathrm{O} & -1.434204 & 1.828396 & -3.508368 \\ \mathrm{H} & -1.541995 & 1.834966 & -4.474072 \\ \mathrm{H} & 1.541995 & -1.834966 & -4.474072 \\ \mathrm{H} & -2.335878 & 1.842997 & -3.123265 \\ \mathrm{O} & 1.434223 & 1.828388 & 3.508433 \\ \mathrm{H} & 2.335879 & 1.843096 & 3.123288 \\ \mathrm{O} & -1.434223 & -1.828388 & 3.508433 \\ \mathrm{H} & -2.335879 & -1.843096 & 3.123288 \\ \mathrm{H} & 1.542060 & 1.834894 & 4.474132 \\ \mathrm{H} & -1.542060 & -1.834894 & 4.474132\end{array}$

$\left[{ }^{1} \mathrm{Sc}\left(\mathrm{H}_{2} \mathrm{O}\right)_{5}(\mathrm{OH})\left(\mathrm{H}_{2} \mathrm{O}\right)_{12}\right]^{2+}$ (a)

$\mathrm{E}=-2136.2699996$

$\begin{array}{lrrr}\mathrm{SC} & -0.155056 & 0.019456 & 0.020636 \\ \mathrm{O} & -2.386811 & 0.171492 & -0.027041 \\ \mathrm{O} & -0.115080 & 1.521955 & 1.616610 \\ \mathrm{H} & -2.904248 & 1.004999 & 0.077801 \\ \mathrm{H} & -3.029647 & -0.562659 & -0.169988 \\ \mathrm{O} & -0.025193 & 1.580883 & -1.494734 \\ \mathrm{O} & -0.300027 & -1.481928 & -1.586731 \\ \mathrm{O} & -0.361042 & -1.572957 & 1.511304 \\ \mathrm{O} & 1.703422 & -0.153261 & 0.079984 \\ \mathrm{H} & 0.750412 & 1.517644 & 2.097046 \\ \mathrm{H} & -0.513111 & 2.420254 & 1.652889 \\ \mathrm{H} & 0.878083 & 1.674585 & -1.894533 \\ \mathrm{H} & -0.553357 & 2.397866 & -1.638600 \\ \mathrm{H} & 0.564614 & -1.627008 & -2.043654 \\ \mathrm{H} & -0.833149 & -2.306901 & -1.621196 \\ \mathrm{H} & 0.498135 & -1.868407 & 1.901210 \\ \mathrm{H} & -1.047530 & -2.265986 & 1.639517 \\ \mathrm{H} & 2.563251 & -0.597868 & 0.041629\end{array}$




$\begin{array}{crrr}\mathrm{O} & -3.607124 & 2.653488 & 0.175049 \\ \mathrm{H} & -4.560368 & 2.778333 & 0.303460 \\ \mathrm{H} & -3.151407 & 3.155612 & 0.883741 \\ \mathrm{O} & -4.002402 & -2.063320 & -0.319233 \\ \mathrm{H} & -4.953852 & -2.027388 & -0.504120 \\ \mathrm{H} & -3.595565 & -2.638518 & -1.001359 \\ \mathrm{O} & 2.559399 & 1.375048 & -2.348091 \\ \mathrm{H} & 2.973418 & 1.903243 & -3.048182 \\ \mathrm{H} & 3.043653 & 1.594134 & -1.507085 \\ \mathrm{O} & 3.743184 & 1.979105 & 0.039282 \\ \mathrm{H} & 4.511345 & 2.516784 & 0.277408 \\ \mathrm{H} & 3.311355 & 1.700981 & 0.868940 \\ \mathrm{O} & 2.229029 & -1.967340 & 2.392136 \\ \mathrm{H} & 2.517639 & -2.563860 & 3.100408 \\ \mathrm{H} & 2.743114 & -2.214366 & 1.590743 \\ \mathrm{O} & -2.000832 & -3.699252 & -1.378491 \\ \mathrm{H} & -1.998215 & -4.453206 & -1.989044 \\ \mathrm{H} & -1.927093 & -4.060099 & -0.471293 \\ \mathrm{O} & -1.447409 & 3.962302 & 1.371000 \\ \mathrm{H} & -1.366899 & 4.704843 & 1.990202 \\ \mathrm{H} & -1.259403 & 4.308879 & 0.474623 \\ \mathrm{O} & 2.400047 & 1.058234 & 2.651937 \\ \mathrm{H} & 2.735165 & 1.305696 & 3.527752 \\ \mathrm{H} & 2.420783 & 0.078688 & 2.613525 \\ \mathrm{O} & 2.289232 & -1.486483 & -2.595760 \\ \mathrm{H} & 2.527385 & -1.789642 & -3.485890 \\ \mathrm{H} & 2.461477 & -0.514822 & -2.577913 \\ \mathrm{O} & 3.621983 & -2.232032 & -0.095024 \\ \mathrm{H} & 4.574806 & -2.396338 & -0.157099 \\ \mathrm{H} & 3.271843 & -2.262813 & -1.008698 \\ \mathrm{O} & -1.634121 & 3.856183 & -1.464888 \\ \mathrm{H} & -1.686877 & 4.501766 & -2.187161 \\ \mathrm{H} & -2.546410 & 3.548440 & -1.288362 \\ \mathrm{O} & -2.345521 & -3.522666 & 1.441254 \\ \mathrm{H} & -2.545365 & -4.129210 & 2.171541 \\ \mathrm{H} & -3.183661 & -3.077846 & 1.199893\end{array}$

$\left[{ }^{1} \mathrm{Sc}\left(\mathrm{H}_{2} \mathrm{O}\right)_{5}(\mathrm{OH})\left(\mathrm{H}_{2} \mathrm{O}\right)_{12}\right]^{2+}(\mathrm{b})$

$\mathrm{E}=-2136.269999$

$\begin{array}{llll}\text { SC } & -0.155355 & 0.019571 & 0.020035\end{array}$

$\begin{array}{llll}\mathrm{O} & -2.387481 & 0.169621 & -0.028187\end{array}$

$\begin{array}{llll}\mathrm{O} & -0.116808 & 1.521916 & 1.616167\end{array}$

$\begin{array}{llll}\mathrm{H} & -2.905918 & 1.002568 & 0.076070\end{array}$

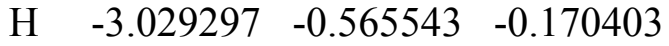

$\begin{array}{lllll}\mathrm{O} & -0.297919 & -1.481781 & -1.587800\end{array}$

$\begin{array}{llll}\mathrm{O} & -0.360151 & -1.573615 & 1.510094\end{array}$ 


\begin{tabular}{|c|c|c|c|}
\hline $\mathrm{O}$ & -0.026462 & 1.581405 & -1.495058 \\
\hline D & 1.703407 & -0.150831 & 0.079528 \\
\hline & 0.749152 & 1.519265 & 2.095670 \\
\hline & -0.516849 & 2.419379 & 1.652922 \\
\hline $\mathrm{H}$ & 0.567098 & -1.626635 & -2.043911 \\
\hline & -0.830368 & -2.307205 & -1.622140 \\
\hline $\mathrm{H}$ & 0.499015 & -1.867493 & 1.901272 \\
\hline $\mathrm{H}$ & -1.045834 & -2.267438 & 1.638332 \\
\hline $\mathrm{H}$ & 0.877087 & 1.675923 & -1.894034 \\
\hline $\mathrm{H}$ & -0.554882 & 2.398341 & -1.637861 \\
\hline $\mathrm{H}$ & 2.562429 & -0.597357 & 0.044350 \\
\hline $\mathrm{O}$ & -3.610877 & 2.650026 & 0.173688 \\
\hline $\mathrm{H}$ & -4.564502 & 2.773196 & 0.300898 \\
\hline $\mathrm{H}$ & -3.156961 & 3.151782 & 0.883740 \\
\hline $\mathrm{O}$ & -3.999883 & -2.067809 & -0.319800 \\
\hline $\mathrm{H}$ & -4.951246 & -2.032493 & -0.505323 \\
\hline $\mathrm{H}$ & -3.592117 & -2.642751 & -1.001592 \\
\hline $\mathrm{O}$ & 2.558267 & 1.378120 & -2.347967 \\
\hline $\mathrm{H}$ & 2.971955 & 1.906645 & -3.047999 \\
\hline $\mathrm{H}$ & 3.042748 & 1.596924 & -1.507013 \\
\hline $\mathrm{O}$ & 3.743243 & 1.981128 & 0.039088 \\
\hline $\mathrm{H}$ & 4.509967 & 2.520556 & 0.277826 \\
\hline $\mathrm{H}$ & 3.310657 & 1.703053 & 0.868352 \\
\hline $\mathrm{O}$ & 2.229155 & -1.965534 & 2.393286 \\
\hline $\mathrm{H}$ & 2.517226 & -2.561517 & 3.102235 \\
\hline $\mathrm{H}$ & 2.744059 & -2.212953 & 1.592576 \\
\hline $\mathrm{O}$ & -1.452540 & 3.959540 & 1.372562 \\
\hline $\mathrm{H}$ & -1.371955 & 4.701500 & 1.992447 \\
\hline $\mathrm{H}$ & -1.265807 & 4.307063 & 0.476259 \\
\hline $\mathrm{O}$ & -1.996275 & -3.701066 & -1.379096 \\
\hline $\mathrm{H}$ & -1.993484 & -4.454787 & -1.989937 \\
\hline $\mathrm{H}$ & -1.921588 & -4.062245 & -0.472112 \\
\hline $\mathrm{O}$ & 2.398762 & 1.060637 & 2.652320 \\
\hline $\mathrm{H}$ & 2.732833 & 1.309128 & 3.528236 \\
\hline $\mathrm{H}$ & 2.420233 & 0.081109 & 2.614824 \\
\hline $\mathrm{O}$ & 2.293393 & -1.483821 & -2.594882 \\
\hline $\mathrm{H}$ & 2.532359 & -1.786640 & -3.484906 \\
\hline $\mathrm{H}$ & 2.463570 & -0.511790 & -2.577184 \\
\hline $\mathrm{O}$ & 3.623293 & -2.229002 & -0.093127 \\
\hline $\mathrm{H}$ & 4.576679 & -2.390457 & -0.154080 \\
\hline $\mathrm{H}$ & 3.274745 & -2.258783 & -1.007458 \\
\hline $\mathrm{O}$ & -1.637230 & 3.855828 & -1.462854 \\
\hline $\mathrm{H}$ & -2.549589 & 3.547399 & -1.287989 \\
\hline $\mathrm{H}$ & -1.689444 & 4.502738 & -2.183987 \\
\hline $\mathrm{O}$ & -2.342300 & -3.525738 & 1.440800 \\
\hline $\mathrm{H}$ & -3.180904 & -3.081870 & 1.199356 \\
\hline
\end{tabular}




\begin{tabular}{|c|c|c|c|}
\hline & -2.541360 & -4.131788 & 71707 \\
\hline \multicolumn{4}{|c|}{$\left[{ }^{1} \mathrm{Sc}\left(\mathrm{H}_{2} \mathrm{O}\right)_{5}(\mathrm{OH})\left(\mathrm{H}_{2} \mathrm{O}\right)_{12}\right]^{2+}(\mathrm{c})$} \\
\hline \multicolumn{4}{|c|}{$E=-2136.267313$} \\
\hline & 2.308180 & -0.261052 & 0.337099 \\
\hline & 3.027721 & -0.100325 & -0.317097 \\
\hline & 2.644814 & -0.099768 & \\
\hline $\mathrm{O}$ & 4.053345 & 0.625910 & -1.6 \\
\hline $\mathrm{H}$ & 4.95 & & \\
\hline $\mathrm{H}$ & 3.55 & 0.5 & \\
\hline & 2.88 & & \\
\hline $\mathrm{H}$ & 3.76 & -0.0 & \\
\hline & 2.25 & & \\
\hline D & 1.9 & & \\
\hline & & & \\
\hline $\mathrm{H}$ & & & \\
\hline $\mathrm{O}$ & 0.9 & 1.4 & \\
\hline $\mathrm{H}$ & $0.5^{\prime}$ & & \\
\hline $\mathrm{H}$ & $1.1^{\prime}$ & 2.4 & \\
\hline $\mathrm{O}$ & & & \\
\hline $\mathrm{H}$ & -0.2 & & \\
\hline $\mathrm{H}$ & & & \\
\hline $\mathrm{O}$ & 2.8 & & \\
\hline $\mathrm{H}$ & & & \\
\hline $\mathrm{H}$ & 3.5 & & \\
\hline $\mathrm{O}$ & -1.5 & & \\
\hline $\mathrm{H}$ & -1.6 & & \\
\hline $\mathrm{H}$ & -2.35 & & \\
\hline $\mathrm{O}$ & -3.7 & & -0 \\
\hline $\mathrm{H}$ & -4.47 & & -1 \\
\hline $\mathrm{H}$ & -4.17 & 2.0 & \\
\hline $\mathrm{O}$ & -5.005630 & & \\
\hline $\mathrm{H}$ & & & \\
\hline $\mathrm{H}$ & -5.48 & & \\
\hline $\mathrm{O}$ & -1.86 & -0.1 & \\
\hline $\mathrm{H}$ & -2.596628 & & -0. \\
\hline $\mathrm{H}$ & -2.247669 & -0.9 & \\
\hline $\mathrm{O}$ & -2.767563 & -2.603678 & -1.771939 \\
\hline $\mathrm{H}$ & -3.415595 & -2.778096 & \\
\hline $\mathrm{H}$ & -1.974492 & -3.148214 & -1.9 \\
\hline $\mathrm{O}$ & -0.187835 & -3.72 & 6699 \\
\hline $\mathrm{H}$ & -0.020116 & -3.596951 & -0.780439 \\
\hline $\mathrm{H}$ & 0.142610 & -4.607963 & -1.978324 \\
\hline $\mathrm{O}$ & -0.110987 & -2.214262 & \\
\hline $\mathrm{H}$ & -1.041082 & -2.436290 & 0.957633 \\
\hline $\mathrm{H}$ & 0.489076 & -2.432512 & 1.440185 \\
\hline
\end{tabular}




$\begin{array}{lrrr}\mathrm{O} & 1.466667 & -2.628692 & 2.751000 \\ \mathrm{H} & 1.817345 & -3.437885 & 3.148097 \\ \mathrm{H} & 2.063769 & -1.889780 & 2.988432 \\ \mathrm{O} & -2.666085 & -2.874295 & 1.059232 \\ \mathrm{H} & -3.115143 & -3.478525 & 1.666904 \\ \mathrm{H} & -3.062988 & -2.982312 & 0.174609 \\ \mathrm{O} & 0.701809 & -1.149119 & -1.972059 \\ \mathrm{H} & 0.562410 & -2.103710 & -2.148497 \\ \mathrm{H} & 1.126863 & -0.717850 & -2.743454 \\ \mathrm{O} & -0.147268 & 0.728693 & 1.593543 \\ \mathrm{H} & -0.046063 & 1.092414 & 2.484256 \\ \mathrm{SC} & 0.166272 & -0.100519 & -0.036821\end{array}$

$\left[{ }^{1} \mathrm{Sc}\left(\mathrm{H}_{2} \mathrm{O}\right)_{5}(\mathrm{OH})\left(\mathrm{H}_{2} \mathrm{O}\right)_{12}\right]^{2+}(\mathrm{d})$

$\mathrm{E}=-2136.274154$

$\begin{array}{rrrr}\mathrm{O} & -1.587113 & -0.717248 & -0.993169 \\ \mathrm{H} & -2.172187 & -0.221388 & -1.600585 \\ \mathrm{O} & -3.094254 & 1.192601 & -2.466195 \\ \mathrm{H} & -3.908795 & 1.084755 & -2.980208 \\ \mathrm{H} & -3.342559 & 1.625641 & -1.621669 \\ \mathrm{O} & -4.047329 & -2.613688 & 0.882107 \\ \mathrm{H} & -4.718425 & -3.256288 & 1.151121 \\ \mathrm{H} & -3.343793 & -3.095136 & 0.401014 \\ \mathrm{O} & -3.207157 & 2.024763 & 0.246961 \\ \mathrm{H} & -3.741627 & 2.761423 & 0.581694 \\ \mathrm{H} & -3.431548 & 1.236685 & 0.805373 \\ \mathrm{O} & -1.821507 & -3.333460 & -0.762522 \\ \mathrm{H} & -2.008666 & -3.888248 & -1.535205 \\ \mathrm{H} & -1.902658 & -2.377394 & -1.044339 \\ \mathrm{O} & 1.072990 & 0.348848 & -1.954937 \\ \mathrm{H} & 2.034319 & 0.275346 & -2.198777 \\ \mathrm{H} & 0.553362 & 0.816612 & -2.669031 \\ \mathrm{O} & -0.524133 & 1.724544 & -3.565109 \\ \mathrm{H} & -0.487534 & 1.867084 & -4.521582 \\ \mathrm{H} & -1.469091 & 1.628199 & -3.320612 \\ \mathrm{O} & 3.699056 & 0.066908 & -2.349493 \\ \mathrm{H} & 4.190781 & -0.033852 & -3.177131 \\ \mathrm{H} & 4.105575 & -0.525091 & -1.688677 \\ \mathrm{O} & 4.288678 & -1.347482 & 0.088943 \\ \mathrm{H} & 5.176251 & -1.242792 & 0.466311 \\ \mathrm{H} & 4.032560 & -2.284548 & 0.228916 \\ \mathrm{O} & 2.980861 & -3.843637 & 0.505594 \\ \mathrm{H} & 3.157599 & -4.567994 & -0.117991 \\ \mathrm{H} & 3.036586 & -4.244974 & 1.388946 \\ \mathrm{O} & 2.063963 & 0.136864 & 0.836394 \\ \mathrm{H} & 2.911999 & -0.325656 & 0.638498\end{array}$




$\begin{array}{cccc}\mathrm{H} & 2.273725 & 0.947192 & 1.358815 \\ \mathrm{O} & 2.370299 & 2.511890 & 2.226081 \\ \mathrm{H} & 3.192084 & 2.828471 & 2.632676 \\ \mathrm{H} & 2.069661 & 3.218151 & 1.614590 \\ \mathrm{O} & 1.235289 & 4.171983 & 0.180063 \\ \mathrm{H} & 0.841200 & 5.025552 & 0.425438 \\ \mathrm{H} & 1.786128 & 4.357418 & -0.598642 \\ \mathrm{O} & -0.713018 & -0.398660 & 1.853863 \\ \mathrm{H} & -0.320940 & 0.102101 & 2.619156 \\ \mathrm{H} & -1.698659 & -0.379832 & 1.956369 \\ \mathrm{O} & -3.453666 & -0.213811 & 1.870327 \\ \mathrm{H} & -3.910444 & -0.123788 & 2.721273 \\ \mathrm{H} & -3.749028 & -1.093206 & 1.493431 \\ \mathrm{O} & 0.309177 & 1.172484 & 3.764212 \\ \mathrm{H} & 0.391523 & 1.052859 & 4.720904 \\ \mathrm{H} & 1.029602 & 1.762544 & 3.473339 \\ \mathrm{O} & -0.476005 & 1.883054 & 0.051828 \\ \mathrm{H} & 0.036892 & 2.708518 & -0.062737 \\ \mathrm{H} & -1.434103 & 2.097923 & 0.181328 \\ \mathrm{O} & 0.627801 & -2.365046 & -0.192198 \\ \mathrm{H} & 1.377598 & -2.900571 & 0.138607 \\ \mathrm{H} & -0.147896 & -2.960330 & -0.379926 \\ \mathrm{SC} & 0.085669 & -0.242031 & -0.155365\end{array}$

$\left[{ }^{1} \mathrm{Sc}\left(\mathrm{H}_{2} \mathrm{O}\right)_{5}(\mathrm{OH})\left(\mathrm{H}_{2} \mathrm{O}\right)_{12}\right]^{2+}(\mathrm{e})$

$\mathrm{E}=-2136.267312$

$\begin{array}{rrrr}\mathrm{O} & 2.307593 & -0.264232 & 0.338419 \\ \mathrm{H} & 2.643690 & -0.104133 & 1.252771 \\ \mathrm{H} & 3.027915 & -0.104339 & -0.315088 \\ \mathrm{O} & 2.875857 & -0.180278 & 3.035004 \\ \mathrm{H} & 3.756726 & -0.097951 & 3.431754 \\ \mathrm{H} & 2.288234 & 0.446728 & 3.518604 \\ \mathrm{O} & 4.055554 & 0.621540 & -1.627276 \\ \mathrm{H} & 4.959782 & 0.328580 & -1.818345 \\ \mathrm{H} & 3.556792 & 0.594905 & -2.473338 \\ \mathrm{O} & 0.985297 & 1.480570 & 4.275263 \\ \mathrm{H} & 0.570150 & 1.141133 & 5.084261 \\ \mathrm{H} & 1.164332 & 2.418656 & 4.447266 \\ \mathrm{O} & 1.989774 & 0.703220 & -3.535990 \\ \mathrm{H} & 1.419662 & 1.346361 & -3.066331 \\ \mathrm{H} & 1.981940 & 0.934035 & -4.477882 \\ \mathrm{O} & -0.114934 & -2.214389 & 0.663325 \\ \mathrm{H} & -1.045780 & -2.435020 & 0.953941 \\ \mathrm{H} & 0.483649 & -2.433814 & 1.438684 \\ \mathrm{O} & 1.458594 & -2.631847 & 2.751138 \\ \mathrm{H} & 1.809157 & -3.441971 & 3.146462\end{array}$




$\begin{array}{cccc}\mathrm{H} & 2.056709 & -1.893868 & 2.989003 \\ \mathrm{O} & -2.671552 & -2.870759 & 1.053535 \\ \mathrm{H} & -3.121700 & -3.475474 & 1.659930 \\ \mathrm{H} & -3.067737 & -2.977337 & 0.168407 \\ \mathrm{O} & -2.769744 & -2.597388 & -1.777561 \\ \mathrm{H} & -3.417413 & -2.770202 & -2.477828 \\ \mathrm{H} & -1.977284 & -3.142928 & -1.977568 \\ \mathrm{O} & -0.191971 & -3.726558 & -1.740490 \\ \mathrm{H} & -0.025107 & -3.596082 & -0.783864 \\ \mathrm{H} & 0.137040 & -4.605936 & -1.982810 \\ \mathrm{O} & -1.864656 & -0.149221 & -0.818665 \\ \mathrm{H} & -2.247784 & -0.926270 & -1.290432 \\ \mathrm{H} & -2.595314 & 0.483375 & -0.602465 \\ \mathrm{O} & -3.748031 & 1.826417 & -0.553316 \\ \mathrm{H} & -4.475581 & 1.761317 & -1.191581 \\ \mathrm{H} & -4.172805 & 2.097103 & 0.302261 \\ \mathrm{O} & -4.995635 & 2.619827 & 1.724384 \\ \mathrm{H} & -5.320108 & 3.519338 & 1.879142 \\ \mathrm{H} & -5.473850 & 2.048521 & 2.343005 \\ \mathrm{O} & 0.589622 & 1.725276 & -1.263376 \\ \mathrm{H} & -0.198253 & 2.335316 & -1.395669 \\ \mathrm{H} & 1.340589 & 2.285948 & -0.919558 \\ \mathrm{O} & 2.855478 & 2.955385 & -0.538767 \\ \mathrm{H} & 3.157713 & 3.874161 & -0.568167 \\ \mathrm{H} & 3.573122 & 2.393310 & -0.889635 \\ \mathrm{O} & -1.552906 & 3.218391 & -1.630696 \\ \mathrm{H} & -1.627962 & 4.171758 & -1.775047 \\ \mathrm{H} & -2.391688 & 2.905089 & -1.228904 \\ \mathrm{O} & -0.147907 & 0.727217 & 1.593796 \\ \mathrm{H} & -0.049807 & 1.088703 & 2.485728 \\ \mathrm{O} & 0.701968 & -1.148237 & -1.972691 \\ \mathrm{H} & 0.561218 & -2.102466 & -2.150090 \\ \mathrm{H} & 1.127998 & -0.716855 & -2.743487 \\ \mathrm{SC} & 0.166311 & -0.100436 & -0.037211 \\ & & & \end{array}$

$\left[{ }^{1} \mathrm{Sc}\left(\mathrm{H}_{2} \mathrm{O}\right)_{5}(\mathrm{OH})\left(\mathrm{H}_{2} \mathrm{O}\right)_{12}\right]^{2+}$ (f)

$\mathrm{E}=-2136.274153$

$\begin{array}{rrrr}\mathrm{O} & 2.063313 & 0.135216 & 0.836477 \\ \mathrm{H} & 2.273117 & 0.944732 & 1.360127 \\ \mathrm{H} & 2.911370 & -0.327208 & 0.638358 \\ \mathrm{O} & 2.370684 & 2.508538 & 2.229093 \\ \mathrm{H} & 3.192233 & 2.824927 & 2.636305 \\ \mathrm{H} & 2.069846 & 3.215514 & 1.618487 \\ \mathrm{O} & 4.288058 & -1.348750 & 0.089002 \\ \mathrm{H} & 5.175617 & -1.244199 & 0.466446 \\ \mathrm{H} & 4.032106 & -2.285937 & 0.228358\end{array}$




\begin{tabular}{|c|c|c|c|}
\hline $\mathrm{O}$ & 1.236842 & 4.170895 & 0.185312 \\
\hline $\mathrm{H}$ & 0.842255 & 5.023905 & 0.431851 \\
\hline $\mathrm{H}$ & 1.788089 & 4.357839 & -0.592750 \\
\hline $\mathrm{O}$ & 2.979355 & -3.845438 & 0.501948 \\
\hline $\mathrm{H}$ & 3.156059 & -4.568478 & -0.123174 \\
\hline $\mathrm{H}$ & 3.034683 & -4.248746 & 1.384429 \\
\hline $\mathrm{O}$ & -0.713975 & -0.400494 & 1.853036 \\
\hline $\mathrm{H}$ & -1.699648 & -0.381298 & 1.955187 \\
\hline $\mathrm{H}$ & -0.322046 & 0.098963 & 2.619239 \\
\hline $\mathrm{O}$ & 0.308069 & 1.169333 & 3.764629 \\
\hline $\mathrm{H}$ & 0.389627 & 1.049175 & 4.721323 \\
\hline $\mathrm{H}$ & 1.029400 & 1.758704 & 3.474582 \\
\hline $\mathrm{O}$ & -3.454748 & -0.214766 & 1.868425 \\
\hline $\mathrm{H}$ & -3.912217 & -0.126566 & 2.719184 \\
\hline $\mathrm{H}$ & -3.749558 & -1.093518 & 1.489723 \\
\hline $\mathrm{O}$ & -4.047197 & -2.615329 & 0.880637 \\
\hline $\mathrm{H}$ & -4.719366 & -3.257543 & 47850 \\
\hline $\mathrm{H}$ & -3.344429 & -3.096424 & 8120 \\
\hline $\mathrm{O}$ & -1.822837 & -3.332625 & -0.766687 \\
\hline $\mathrm{H}$ & -2.009485 & -3.886288 & 0302 \\
\hline $\mathrm{H}$ & -1.903860 & -2.376178 & -1.047174 \\
\hline $\mathrm{O}$ & -1.587997 & -0.716100 & 3903 \\
\hline $\mathrm{H}$ & -2.171818 & -0.219138 & -1.601617 \\
\hline $\mathrm{O}$ & -3.091451 & 1.197390 & -2.466464 \\
\hline $\mathrm{H}$ & -3.905351 & 1.089907 & 1576 \\
\hline $\mathrm{H}$ & -3.340904 & 1.629014 & -1.621548 \\
\hline $\mathrm{O}$ & -3.206367 & 2.025511 & 0.247777 \\
\hline $\mathrm{H}$ & -3.740569 & 2.762195 & 0.582903 \\
\hline $\mathrm{H}$ & -3.431379 & 1.237059 & 0.805444 \\
\hline $\mathrm{O}$ & 1.072577 & 0.347037 & -1.956097 \\
\hline $\mathrm{H}$ & 0.554530 & 0.820017 & -2.667882 \\
\hline $\mathrm{H}$ & 2.034372 & 0.275152 & -2.198565 \\
\hline $\mathrm{O}$ & 3.699651 & 0.069107 & -2.347646 \\
\hline $\mathrm{H}$ & 4.191486 & -0.030622 & -3.175351 \\
\hline $\mathrm{H}$ & 4.105708 & -0.524208 & -1.687734 \\
\hline $\mathrm{O}$ & -0.520809 & 1.735095 & -3.559595 \\
\hline $\mathrm{H}$ & -0.483319 & 1.881663 & -4.515438 \\
\hline $\mathrm{H}$ & -1.466016 & 1.636697 & -3.316799 \\
\hline $\mathrm{O}$ & -0.474993 & 1.882671 & 0.052391 \\
\hline $\mathrm{H}$ & -1.432928 & 2.097864 & 0.182342 \\
\hline $\mathrm{H}$ & 0.038437 & 2.708054 & -0.060308 \\
\hline $\mathrm{O}$ & 0.626529 & -2.365674 & -0.194339 \\
\hline $\mathrm{H}$ & -0.149267 & -2.960569 & -0.382965 \\
\hline $\mathrm{H}$ & 1.376277 & -2.901710 & 0.135730 \\
\hline $\mathrm{SC}$ & 0.085205 & -0.242652 & -0.155941 \\
\hline
\end{tabular}




\begin{tabular}{|c|c|c|c|}
\hline \multicolumn{4}{|c|}{$\begin{array}{l}{\left[{ }^{1} \mathrm{Sc}\left(\mathrm{H}_{2} \mathrm{O}\right)_{6}\right]^{3+}} \\
\mathrm{E}=-1218.5071573\end{array}$} \\
\hline & 0.000006 & 0.000000 & 0.000006 \\
\hline $\mathrm{O}$ & 0.633684 & 1.850431 & -0.941159 \\
\hline $\mathrm{O}$ & 1.753366 & 0.050368 & 1.278392 \\
\hline $\mathrm{O}$ & -0.633696 & -1.850442 & 0.941132 \\
\hline $\mathrm{O}$ & -1.753363 & -0.050365 & -1.278373 \\
\hline $\mathrm{O}$ & 1.112095 & -1.133796 & -1.479249 \\
\hline $\mathrm{O}$ & -1.112098 & 1.133804 & 1.479244 \\
\hline $\mathrm{H}$ & 0.786539 & -1.463560 & -2.344038 \\
\hline $\mathrm{H}$ & 2.047358 & -1.425145 & -1.425067 \\
\hline $\mathrm{H}$ & 2.006404 & -0.600961 & 1.967109 \\
\hline $\mathrm{H}$ & 2.461505 & 0.729341 & 1.290013 \\
\hline $\mathrm{H}$ & 1.206786 & 1.950200 & -1.731222 \\
\hline $\mathrm{H}$ & 0.407320 & 2.764821 & -0.666899 \\
\hline $\mathrm{H}$ & -2.461707 & -0.729129 & -1.289717 \\
\hline $\mathrm{H}$ & -2.006206 & 0.600763 & -1.967351 \\
\hline $\mathrm{H}$ & -0.407134 & -2.764819 & 0.666996 \\
\hline $\mathrm{H}$ & -1.206966 & -1.950235 & 1.731071 \\
\hline $\mathrm{H}$ & -0.786762 & 1.462988 & 2.344336 \\
\hline $\mathrm{H}$ & -2.047163 & 1.425726 & 1.424747 \\
\hline
\end{tabular}

$\begin{array}{crrr}{\left[{ }^{1} \mathrm{Sc}\left(\mathrm{H}_{2} \mathrm{O}\right)_{5}(\mathrm{OH})\right]^{2+}(\mathrm{a})} \\ \mathrm{E}=-1218.447616 \\ \mathrm{SC} & 0.000063 & 0.000009 & -0.204279 \\ \mathrm{O} & -2.077554 & -0.741280 & -0.066263 \\ \mathrm{O} & 2.077725 & 0.741025 & -0.065479 \\ \mathrm{H} & -2.593407 & -0.944698 & -0.868090 \\ \mathrm{H} & -2.652359 & -0.920541 & 0.698642 \\ \mathrm{O} & 0.000388 & -0.000063 & -2.027164 \\ \mathrm{H} & 2.593801 & 0.944342 & -0.867191 \\ \mathrm{H} & 2.652301 & 0.920456 & 0.699558 \\ \mathrm{H} & 0.000462 & -0.000130 & -2.995317 \\ \mathrm{O} & 0.751361 & -2.082602 & 0.172407 \\ \mathrm{H} & 0.194928 & -2.880644 & 0.126381 \\ \mathrm{H} & 1.649409 & -2.373600 & -0.068220 \\ \mathrm{O} & -0.751379 & 2.082708 & 0.171855 \\ \mathrm{H} & -0.194881 & 2.880743 & 0.126570 \\ \mathrm{H} & -1.649172 & 2.373791 & -0.069627 \\ \mathrm{O} & -0.000635 & 0.000174 & 2.085983 \\ \mathrm{H} & 0.297276 & -0.720467 & 2.668349 \\ \mathrm{H} & -0.298928 & 0.720858 & 2.668097\end{array}$

$\left[{ }^{1} \mathrm{Sc}\left(\mathrm{H}_{2} \mathrm{O}\right)_{5}(\mathrm{OH})\right]^{2+}(\mathrm{b})$

$\mathrm{E}=-1218.447594$

$\begin{array}{llll}\text { SC } & -0.001760 & 0.003297 & -0.198646\end{array}$ 


$\begin{array}{crcc}\mathrm{O} & 1.809808 & 1.265124 & -0.074690 \\ \mathrm{O} & -1.799552 & -1.276006 & -0.052793 \\ \mathrm{H} & 2.347072 & 1.564003 & 0.679951 \\ \mathrm{H} & 2.223188 & 1.623488 & -0.881658 \\ \mathrm{O} & -0.026051 & 0.008120 & -2.021482 \\ \mathrm{H} & -2.232333 & -1.626030 & -0.852991 \\ \mathrm{H} & -2.321537 & -1.577077 & 0.711623 \\ \mathrm{H} & -0.036811 & 0.010511 & -2.989572 \\ \mathrm{O} & 0.014438 & -0.007947 & 2.093056 \\ \mathrm{H} & 0.521918 & -0.607209 & 2.668087 \\ \mathrm{H} & -0.495375 & 0.573339 & 2.684211 \\ \mathrm{O} & -1.288378 & 1.803371 & 0.172985 \\ \mathrm{H} & -2.192281 & 1.875852 & -0.184024 \\ \mathrm{H} & -0.969824 & 2.717655 & 0.272702 \\ \mathrm{O} & 1.291444 & -1.799250 & 0.140829 \\ \mathrm{H} & 2.210846 & -1.853552 & -0.177717 \\ \mathrm{H} & 0.968447 & -2.717500 & 0.177717\end{array}$

$\left[{ }^{1} \mathrm{Sc}\left(\mathrm{H}_{2} \mathrm{O}\right)_{5}(\mathrm{OH})\right]^{2+}(\mathrm{c})$

$\mathrm{E}=-1218.447622$

$\begin{array}{lrrr}\mathrm{SC} & 0.000091 & 0.000027 & -0.204492 \\ \mathrm{O} & -2.084181 & 0.722575 & -0.066880 \\ \mathrm{O} & 2.084416 & -0.721995 & -0.065834 \\ \mathrm{H} & -2.602053 & 0.919803 & -0.868954 \\ \mathrm{H} & -2.660270 & 0.898425 & 0.697846 \\ \mathrm{O} & 0.001038 & 0.000359 & -2.027363 \\ \mathrm{H} & 2.603089 & -0.918286 & -0.867610 \\ \mathrm{H} & 2.659836 & -0.898375 & 0.699276 \\ \mathrm{H} & 0.001509 & 0.000627 & -2.995512 \\ \mathrm{O} & -0.732463 & -2.089317 & 0.172221 \\ \mathrm{H} & -0.170076 & -2.882662 & 0.117486 \\ \mathrm{H} & -1.630573 & -2.386806 & -0.060098 \\ \mathrm{O} & 0.731707 & 2.089298 & 0.173941 \\ \mathrm{H} & 0.169197 & 2.882575 & 0.120128 \\ \mathrm{H} & 1.629678 & 2.387254 & -0.058304 \\ \mathrm{O} & -0.000586 & -0.000983 & 2.085699 \\ \mathrm{H} & 0.287184 & 0.723467 & 2.668438 \\ \mathrm{H} & -0.288884 & -0.726088 & 2.667362\end{array}$

$\left[{ }^{1} \mathrm{Sc}\left(\mathrm{H}_{2} \mathrm{O}\right)_{5}(\mathrm{OH})\right]^{2+}(\mathrm{d})$

$\mathrm{E}=-1218.447609$

$\begin{array}{lccc}\mathrm{SC} & -0.000439 & 0.000442 & -0.205713 \\ \mathrm{O} & -2.085435 & 0.717567 & -0.059982 \\ \mathrm{O} & 2.082947 & -0.723421 & -0.069849 \\ \mathrm{H} & -2.671213 & 0.859187 & 0.704398 \\ \mathrm{H} & -2.599655 & 0.932092 & -0.859957\end{array}$ 


$\begin{array}{crcc}\mathrm{O} & -0.005285 & 0.004668 & -2.028616 \\ \mathrm{H} & 2.671974 & -0.866966 & 0.691714 \\ \mathrm{H} & 2.593134 & -0.938306 & -0.872260 \\ \mathrm{H} & -0.006725 & 0.007369 & -2.996758 \\ \mathrm{O} & 0.004516 & 0.000807 & 2.085754 \\ \mathrm{H} & -0.309760 & -0.711822 & 2.669345 \\ \mathrm{H} & 0.321625 & 0.713628 & 2.667561 \\ \mathrm{O} & 0.733364 & 2.087804 & 0.171362 \\ \mathrm{H} & 1.595214 & 2.410424 & -0.148795 \\ \mathrm{H} & 0.162282 & 2.874781 & 0.227301 \\ \mathrm{O} & -0.729828 & -2.087920 & 0.170876 \\ \mathrm{H} & -1.590960 & -2.411305 & -0.149639 \\ \mathrm{H} & -0.158938 & -2.874393 & 0.230711\end{array}$

$\left[{ }^{1} \mathrm{Sc}\left(\mathrm{H}_{2} \mathrm{O}\right)_{5}(\mathrm{OH})\right]^{2+}(\mathrm{e})$

$\mathrm{E}=-1218.447505$

$\begin{array}{lrcc}\mathrm{O} & 2.158821 & -0.452173 & -0.075195 \\ \mathrm{H} & 2.751122 & -0.577492 & 0.687115 \\ \mathrm{H} & 2.697864 & -0.560172 & -0.880274 \\ \mathrm{O} & 0.001954 & -0.010701 & 2.083026 \\ \mathrm{H} & -0.163491 & -0.778956 & 2.657141 \\ \mathrm{H} & 0.163877 & 0.746809 & 2.672204 \\ \mathrm{O} & 0.451576 & 2.168158 & 0.193221 \\ \mathrm{H} & -0.192578 & 2.885385 & 0.053088 \\ \mathrm{H} & 1.327577 & 2.569333 & 0.049486 \\ \mathrm{O} & -2.161888 & 0.439887 & -0.066637 \\ \mathrm{H} & -2.703680 & 0.559415 & -0.868208 \\ \mathrm{H} & -2.750762 & 0.558407 & 0.699421 \\ \mathrm{O} & -0.450226 & -2.166834 & 0.170165 \\ \mathrm{H} & 0.198031 & -2.882146 & 0.039511 \\ \mathrm{H} & -1.321357 & -2.568980 & 0.001330 \\ \mathrm{SC} & 0.000176 & 0.003338 & -0.205737 \\ \mathrm{O} & -0.001452 & 0.015215 & -2.028759 \\ \mathrm{H} & -0.000586 & 0.029887 & -2.996906\end{array}$

$\left[{ }^{1} \mathrm{Sc}\left(\mathrm{H}_{2} \mathrm{O}\right)_{5}(\mathrm{OH})\right]^{2+}(\mathrm{f})$

$\mathrm{E}=-1218.447616$

$\begin{array}{llll}\mathrm{SC} & 0.000386 & 0.000264 & -0.203291\end{array}$

$\begin{array}{llll}\mathrm{O} & -2.079115 & 0.736759 & -0.069456\end{array}$

$\begin{array}{llll}\mathrm{O} & 2.078441 & -0.738536 & -0.063428\end{array}$

$\begin{array}{llll}\mathrm{H} & -2.659688 & 0.906924 & 0.693187\end{array}$

$\begin{array}{llll}\mathrm{H} & -2.591084 & 0.943777 & -0.872824\end{array}$

$\begin{array}{llll}\mathrm{O} & 0.004790 & 0.001108 & -2.026316\end{array}$

$\begin{array}{llll}\mathrm{H} & 2.656535 & -0.907808 & 0.701252\end{array}$

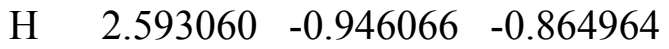

$\begin{array}{llll}\mathrm{H} & 0.006866 & 0.000417 & -2.994461\end{array}$ 


$\begin{array}{cccc}\mathrm{O} & -0.749309 & -2.083645 & 0.169337 \\ \mathrm{H} & -0.188059 & -2.879390 & 0.151786 \\ \mathrm{H} & -1.636766 & -2.382086 & -0.099872 \\ \mathrm{O} & 0.747256 & 2.084380 & 0.172179 \\ \mathrm{H} & 1.630957 & 2.385987 & -0.106068 \\ \mathrm{H} & 0.184899 & 2.879479 & 0.169350 \\ \mathrm{O} & -0.002179 & -0.000533 & 2.086866 \\ \mathrm{H} & -0.310219 & -0.717859 & 2.668044 \\ \mathrm{H} & 0.306323 & 0.714812 & 2.670233\end{array}$

\begin{tabular}{cccc}
\multicolumn{5}{c}{$\left.{ }^{1} \mathrm{Y}\left(\mathrm{H}_{2} \mathrm{O}\right)_{24}\right]^{3+}$} & & \\
$\mathrm{E}=$ & -1873.001021 & & \\
$\mathrm{Y}$ & -0.062314 & -0.008654 & -0.002538 \\
$\mathrm{O}$ & -0.513132 & -2.236093 & -0.672811 \\
$\mathrm{H}$ & -1.211492 & -2.524352 & -1.302305 \\
$\mathrm{H}$ & 0.027209 & -3.040312 & -0.449511 \\
$\mathrm{O}$ & 1.039267 & -1.077088 & 1.812635 \\
$\mathrm{H}$ & 0.617839 & -1.362389 & 2.669662 \\
$\mathrm{H}$ & 1.942694 & -1.440733 & 1.823246 \\
$\mathrm{O}$ & 2.011047 & -0.934947 & -0.962343 \\
$\mathrm{H}$ & 2.874556 & -0.475038 & -1.072078 \\
$\mathrm{H}$ & 2.095935 & -1.774092 & -1.454336 \\
$\mathrm{O}$ & 1.660819 & 1.548260 & 0.658030 \\
$\mathrm{H}$ & 1.693797 & 2.477871 & 0.333596 \\
$\mathrm{H}$ & 2.511020 & 1.362823 & 1.112185 \\
$\mathrm{O}$ & -1.078605 & 2.088316 & 0.642830 \\
$\mathrm{H}$ & -1.962427 & 2.382419 & 0.334206 \\
$\mathrm{H}$ & -0.777586 & 2.751572 & 1.293204 \\
$\mathrm{O}$ & 0.248585 & 1.182250 & -2.023566 \\
$\mathrm{H}$ & -0.139168 & 2.095710 & -2.097171 \\
$\mathrm{H}$ & 1.152834 & 1.270231 & -2.419758 \\
$\mathrm{O}$ & -2.169310 & 0.118533 & -1.197830 \\
$\mathrm{H}$ & -2.185073 & 0.132773 & -2.174135 \\
$\mathrm{H}$ & -2.944811 & -0.435139 & -0.916690 \\
$\mathrm{O}$ & -1.741424 & -0.547786 & 1.599509 \\
$\mathrm{H}$ & -2.420425 & 0.089884 & 1.941234 \\
$\mathrm{H}$ & -2.036681 & -1.436793 & 1.875288 \\
$\mathrm{O}$ & -2.523802 & -2.969169 & -2.535881 \\
$\mathrm{H}$ & -2.595921 & -3.842637 & -2.951311 \\
$\mathrm{H}$ & -2.547657 & -2.304434 & -3.257748 \\
$\mathrm{O}$ & 1.094984 & -4.341663 & -0.278928 \\
$\mathrm{H}$ & 1.727306 & -4.372108 & -1.022757 \\
$\mathrm{H}$ & 0.947326 & -5.250067 & 0.021294 \\
$\mathrm{O}$ & 0.004626 & -2.081182 & 4.060209 \\
$\mathrm{H}$ & 0.136216 & -1.911357 & 5.003663 \\
$\mathrm{H}$ & -0.889075 & -2.463981 & 3.954467
\end{tabular}




$\begin{array}{crrr}\mathrm{O} & 3.877775 & -1.647087 & 2.656344 \\ \mathrm{H} & 3.800795 & -1.752153 & 3.619159 \\ \mathrm{H} & 4.473442 & -2.360642 & 2.374991 \\ \mathrm{O} & 4.474914 & 0.404479 & -1.142124 \\ \mathrm{H} & 5.326915 & 0.087217 & -1.479435 \\ \mathrm{H} & 4.622481 & 0.678395 & -0.208603 \\ \mathrm{O} & 2.758015 & -3.484602 & -2.399077 \\ \mathrm{H} & 3.700812 & -3.715564 & -2.373225 \\ \mathrm{H} & 2.484051 & -3.660203 & -3.313921 \\ \mathrm{O} & 1.445634 & 4.268356 & -0.017992 \\ \mathrm{H} & 2.181613 & 4.874666 & -0.197477 \\ \mathrm{H} & 1.001770 & 4.586659 & 0.797326 \\ \mathrm{O} & 4.265272 & 0.910575 & 1.590624 \\ \mathrm{H} & 4.261538 & 0.046201 & 2.063654 \\ \mathrm{H} & 4.838269 & 1.505385 & 2.100263 \\ \mathrm{O} & -3.794850 & 2.917825 & 0.163975 \\ \mathrm{H} & -4.186310 & 2.585918 & -0.659308 \\ \mathrm{H} & -4.074890 & 3.845628 & 0.223232 \\ \mathrm{O} & -0.196499 & 4.439762 & 2.258016 \\ \mathrm{H} & 0.188431 & 4.333695 & 3.143434 \\ \mathrm{H} & -0.820529 & 5.179327 & 2.344194 \\ \mathrm{O} & -0.549655 & 3.763258 & -2.029943 \\ \mathrm{H} & 0.135829 & 4.142143 & -1.442666 \\ \mathrm{H} & -0.615744 & 4.346950 & -2.800243 \\ \mathrm{O} & 2.733680 & 1.753049 & -2.948726 \\ \mathrm{H} & 3.513113 & 1.458495 & -2.439500 \\ \mathrm{H} & 3.044910 & 2.057692 & -3.813056 \\ \mathrm{O} & -2.570057 & -0.587026 & -4.073156 \\ \mathrm{H} & -1.887077 & -0.389671 & -4.734423 \\ \mathrm{H} & -3.411858 & -0.371468 & -4.507863 \\ \mathrm{O} & -4.145701 & -1.633785 & -0.659880 \\ \mathrm{H} & -3.919372 & -2.356050 & -1.276692 \\ \mathrm{H} & -5.107225 & -1.629456 & -0.549365 \\ \mathrm{O} & -3.660319 & 1.174993 & 2.429864 \\ \mathrm{H} & -4.281895 & 1.186117 & 3.171328 \\ \mathrm{H} & -3.980977 & 1.812287 & 1.764517 \\ \mathrm{H} & -2.539963 & -2.952901 & 3.136106 \\ & -2.584628 & -3.880159 & 2.852758 \\ & -3.340054 & -2.814386 & 3.668566\end{array}$

$$
\begin{aligned}
& {\left[{ }^{1} \mathrm{Y}\left(\mathrm{H}_{2} \mathrm{O}\right)_{23}(\mathrm{OH})\right]^{2+} \text { (a) }} \\
& \mathrm{E}=-1872.771598 \\
& \begin{array}{llll}
\text { Y } & 0.320785 & -0.264764 & -0.106619
\end{array} \\
& \begin{array}{llll}
\mathrm{O} & 0.382183 & 2.029468 & -0.789635
\end{array} \\
& \begin{array}{llll}
\mathrm{O} & 2.525224 & 0.375163 & -0.496819
\end{array} \\
& \text { H } \quad 1.095078 \quad 2.564822 \quad-0.370666
\end{aligned}
$$




$\begin{array}{cccc}\mathrm{H} & -0.239146 & 2.668227 & -1.202184 \\ \mathrm{O} & -1.314070 & -1.878003 & 0.512677 \\ \mathrm{O} & -1.394228 & -0.125055 & -1.751157 \\ \mathrm{O} & 0.067678 & 0.527615 & 1.887822 \\ \mathrm{H} & 3.302002 & -0.058635 & -0.079276 \\ \mathrm{H} & 2.880230 & 1.010178 & -1.170205 \\ \mathrm{H} & -2.287167 & -1.802351 & 0.308652 \\ \mathrm{H} & -1.263520 & -2.380677 & 1.355870 \\ \mathrm{H} & -1.753600 & -0.969720 & -2.115988 \\ \mathrm{H} & -2.028520 & 0.586781 & -1.998287 \\ \mathrm{H} & -0.011691 & 1.459249 & 2.150109 \\ \mathrm{O} & -3.901874 & -1.952524 & -0.064881 \\ \mathrm{H} & -3.924639 & -2.229438 & -0.996563 \\ \mathrm{H} & -4.643987 & -1.344592 & 0.108228 \\ \mathrm{O} & 5.031577 & -0.365909 & 0.280757 \\ \mathrm{H} & 5.377864 & -0.851804 & 1.043519 \\ \mathrm{H} & 5.229102 & 0.582516 & 0.437153 \\ \mathrm{O} & 2.185383 & 3.583400 & 0.750317 \\ \mathrm{H} & 2.307531 & 4.494001 & 0.440558 \\ \mathrm{H} & 1.512448 & 3.632540 & 1.472352 \\ \mathrm{O} & -2.445021 & -2.595397 & -2.489290 \\ \mathrm{H} & -2.656638 & -2.837693 & -3.403301 \\ \mathrm{H} & -1.812064 & -3.276667 & -2.166480 \\ \mathrm{H} & -0.318147 & -4.296630 & -1.653537 \\ \mathrm{O} & -0.909632 & -3.339204 & 2.867988 \\ \mathrm{H} & -1.610184 & -3.691929 & 3.435953 \\ \mathrm{H} & -0.434240 & -2.626822 & 3.375327 \\ \mathrm{O} & -1.492893 & 3.910071 & -1.662686 \\ \mathrm{H} & -1.284941 & 4.607876 & -2.302545 \\ \mathrm{H} & -2.166889 & 3.315627 & -2.082002 \\ \mathrm{O} & -3.204382 & 1.924511 & -2.279106 \\ \mathrm{H} & -3.810201 & 1.914885 & -3.035486 \\ \mathrm{H} & -3.760507 & 2.001433 & -1.444272 \\ \mathrm{O} & -4.399972 & 2.257768 & 0.023893 \\ \mathrm{H} & -4.861984 & 1.559564 & 0.525736 \\ \mathrm{H} & -3.858792 & 2.794476 & 0.632545 \\ \mathrm{H} & -0.022213 & 3.515116 & 2.427978 \\ \mathrm{H} & -0.064461 & 3.830831 & 3.342835 \\ \mathrm{H} & -2.378141 & 3.789003 & 1.997613 \\ \mathrm{H} & 4.180535 & 0.991936 \\ \mathrm{H} & 5.017196 & 1.219146 \\ \mathrm{H} & 4.267518 & 0.054461 \\ \mathrm{H} & 2.493367 & 0.506785 \\ \mathrm{H} & -2.112228 & -2.149691\end{array}$




$\begin{array}{lrrr}\mathrm{H} & -0.103431 & -4.495273 & -0.711596 \\ \mathrm{O} & 0.676376 & -4.554650 & 0.973814 \\ \mathrm{H} & 1.177488 & -5.349191 & 1.213725 \\ \mathrm{H} & 0.080851 & -4.354129 & 1.734563 \\ \mathrm{O} & -5.910322 & -0.041711 & 0.904003 \\ \mathrm{H} & -6.069107 & -0.293183 & 1.827888 \\ \mathrm{H} & -6.792993 & 0.036652 & 0.507232 \\ \mathrm{O} & 3.702295 & 2.257252 & -2.037471 \\ \mathrm{H} & 4.081507 & 2.207170 & -2.926305 \\ \mathrm{H} & 4.415243 & 2.523552 & -1.425677 \\ \mathrm{O} & 1.132925 & -1.895615 & -1.688994 \\ \mathrm{H} & 0.731633 & -2.777434 & -1.840749 \\ \mathrm{H} & 2.109065 & -1.979308 & -1.815683 \\ \mathrm{O} & 3.894557 & -2.158349 & -1.852983 \\ \mathrm{H} & 4.446635 & -2.534916 & -2.552629 \\ \mathrm{H} & 4.478701 & -1.614514 & -1.294190 \\ \mathrm{O} & 1.627345 & -1.841252 & 1.197522 \\ \mathrm{H} & 1.543567 & -2.800355 & 1.018376 \\ \mathrm{H} & 1.517090 & -1.722857 & 2.170181 \\ \mathrm{H} & 0.301172 & -0.427026 & 3.072188 \\ \mathrm{O} & 0.562263 & -1.251082 & 3.637963 \\ \mathrm{H} & 0.905838 & -0.955147 & 4.492199\end{array}$

$\left[{ }^{1} \mathrm{Y}\left(\mathrm{H}_{2} \mathrm{O}\right)_{23}(\mathrm{OH})\right]^{2+}(\mathrm{b})$

$\mathrm{E}=-1872.773581$

$\begin{array}{rrrr}\mathrm{Y} & 0.149001 & 0.222004 & -0.508268 \\ \mathrm{O} & 0.510736 & -2.107309 & -0.940840 \\ \mathrm{O} & -1.842272 & -0.576121 & -1.445848 \\ \mathrm{H} & 1.283426 & -2.345483 & -1.503679 \\ \mathrm{H} & -0.094847 & -2.886111 & -0.889196 \\ \mathrm{O} & -1.461547 & 1.126065 & 0.994681 \\ \mathrm{O} & -0.616484 & 2.163893 & -1.680579 \\ \mathrm{O} & 1.752616 & 0.243800 & -1.952587 \\ \mathrm{H} & -1.966741 & -1.322019 & -2.093630 \\ \mathrm{H} & -2.738827 & -0.198570 & -1.298047 \\ \mathrm{H} & -1.721114 & 2.099790 & 1.003633 \\ \mathrm{H} & -1.934745 & 0.727064 & 1.755973 \\ \mathrm{H} & -1.549477 & 2.446025 & -1.801341 \\ \mathrm{H} & -0.081762 & 2.493302 & -2.438298 \\ \mathrm{H} & 2.143622 & 1.047510 & -2.323222 \\ \mathrm{O} & -2.209488 & 3.626578 & 1.006936 \\ \mathrm{H} & -1.436432 & 4.219449 & 1.013507 \\ \mathrm{H} & -2.676930 & 3.742097 & 0.157395 \\ \mathrm{O} & -2.447309 & -2.683658 & -2.936186 \\ \mathrm{H} & -2.407181 & -2.945242 & -3.865981 \\ \mathrm{H} & -2.148301 & -3.441563 & -2.389850\end{array}$




\begin{tabular}{|c|c|c|c|}
\hline $\mathrm{O}$ & -1.370743 & -4.129637 & -0.843861 \\
\hline $\mathrm{H}$ & -1.210203 & -5.081373 & -0.761007 \\
\hline & -1.974157 & -3.872346 & -0.088609 \\
\hline $\mathrm{O}$ & -2.936744 & -0.011203 & 3.117525 \\
\hline $\mathrm{H}$ & -3.119899 & 0.542562 & 3.891900 \\
\hline $\mathrm{H}$ & -2.327273 & -0.733160 & 3.403449 \\
\hline $\mathrm{O}$ & -1.104364 & -2.020843 & 3.183307 \\
\hline $\mathrm{H}$ & -0.746122 & -2.527079 & 3.928103 \\
\hline $\mathrm{H}$ & -0.339097 & -1.648280 & 2.684167 \\
\hline $\mathrm{O}$ & -4.423619 & 0.405904 & -1.032001 \\
\hline $\mathrm{H}$ & -5.053132 & 0.062258 & -1.685049 \\
\hline $\mathrm{H}$ & -4.661974 & -0.025260 & -0.173359 \\
\hline $\mathrm{O}$ & -2.831072 & -3.276088 & 1.201575 \\
\hline $\mathrm{H}$ & -3.590844 & -2.667886 & 1.133331 \\
\hline $\mathrm{H}$ & -2.292912 & -2.965368 & 1.953529 \\
\hline $\mathrm{O}$ & 0.921927 & -0.816663 & 1.587683 \\
\hline $\mathrm{H}$ & 1.561887 & -1.573977 & 1.464142 \\
\hline $\mathrm{H}$ & 1.382076 & -0.221261 & 2.250357 \\
\hline $\mathrm{O}$ & 2.856296 & -2.677433 & 1.542595 \\
\hline $\mathrm{H}$ & 2.837744 & -3.586152 & 1.872481 \\
\hline $\mathrm{H}$ & 3.570385 & -2.635213 & 0.865300 \\
\hline $\mathrm{O}$ & 2.299648 & 0.690524 & 3.325575 \\
\hline $\mathrm{H}$ & 2.685237 & 0.297942 & 4.121899 \\
\hline $\mathrm{H}$ & 3.040337 & 1.101505 & 2.825352 \\
\hline $\mathrm{O}$ & 4.009322 & 1.856237 & 1.473549 \\
\hline $\mathrm{H}$ & 4.499336 & 2.668991 & 1.668076 \\
\hline $\mathrm{H}$ & 4.653897 & 1.251990 & 1.003192 \\
\hline $\mathrm{O}$ & 5.665882 & 0.231012 & 0.177985 \\
\hline $\mathrm{H}$ & 5.413053 & -0.683830 & -0.078104 \\
\hline $\mathrm{H}$ & 6.631872 & 0.267860 & 0.205220 \\
\hline $\mathrm{O}$ & 4.723028 & -2.326091 & -0.501300 \\
\hline $\mathrm{H}$ & 5.382896 & -3.006694 & -0.703235 \\
\hline $\mathrm{H}$ & 4.127362 & -2.254634 & -1.297087 \\
\hline $\mathrm{O}$ & -3.318293 & 2.949706 & -1.532157 \\
\hline $\mathrm{H}$ & -3.844616 & 3.484923 & -2.144980 \\
\hline $\mathrm{H}$ & -3.851326 & 2.147470 & -1.328878 \\
\hline $\mathrm{O}$ & -4.785848 & -1.027778 & 1.312799 \\
\hline $\mathrm{H}$ & -5.673514 & -1.238058 & 1.642327 \\
\hline $\mathrm{H}$ & -4.295678 & -0.615719 & 2.061451 \\
\hline $\mathrm{O}$ & 0.477260 & 4.606565 & 1.466624 \\
\hline $\mathrm{H}$ & 0.865872 & 5.406732 & 1.079239 \\
\hline $\mathrm{H}$ & 0.514978 & 4.740210 & 2.427456 \\
\hline $\mathrm{O}$ & 1.098806 & 2.907118 & -3.727666 \\
\hline $\mathrm{H}$ & 1.434156 & 3.801016 & -3.894022 \\
\hline $\mathrm{H}$ & 1.013934 & 2.491113 & -4.598983 \\
\hline $\mathrm{O}$ & 1.437709 & 2.014951 & 0.466688 \\
\hline
\end{tabular}




$\begin{array}{rrrc}\mathrm{H} & 1.078351 & 2.855742 & 0.811830 \\ \mathrm{H} & 2.393708 & 1.992650 & 0.709562 \\ \mathrm{H} & 2.504121 & -1.046664 & -2.351760 \\ \mathrm{O} & 2.839791 & -2.016024 & -2.398355 \\ \mathrm{H} & 2.945910 & -2.252428 & -3.331232\end{array}$

$\left[{ }^{1} \mathrm{Y}\left(\mathrm{H}_{2} \mathrm{O}\right)_{23}(\mathrm{OH})\right]^{2+}(\mathrm{c})$

$\mathrm{E}=-1872.749462$

$\begin{array}{rrrc}\mathrm{Y} & -0.114814 & 0.027465 & 0.033678 \\ \mathrm{O} & 0.606605 & 0.941287 & -2.173676 \\ \mathrm{O} & -0.208505 & -2.296511 & -0.744258 \\ \mathrm{H} & 0.281664 & 1.881628 & -2.286895 \\ \mathrm{H} & 1.588706 & 1.005525 & -2.307254 \\ \mathrm{O} & 0.590693 & -0.747916 & 2.169141 \\ \mathrm{O} & -2.036574 & -0.874235 & 1.186691 \\ \mathrm{O} & 1.609955 & 1.766529 & 0.599765 \\ \mathrm{H} & 0.264312 & -3.091742 & -0.400326 \\ \mathrm{H} & -0.731783 & -2.591149 & -1.520403 \\ \mathrm{H} & -0.023893 & -1.143546 & 2.843888 \\ \mathrm{H} & 1.496311 & -0.887859 & 2.494542 \\ \mathrm{H} & -2.845393 & -0.308264 & 1.317257 \\ \mathrm{H} & -2.336272 & -1.798485 & 1.195403 \\ \mathrm{H} & 1.258794 & 2.682365 & 0.521748 \\ \mathrm{H} & 2.332024 & 1.787382 & 1.256805 \\ \mathrm{O} & -0.873235 & -2.024871 & 4.039365 \\ \mathrm{H} & -1.208770 & -1.637014 & 4.859815 \\ \mathrm{H} & -1.623051 & -2.509975 & 3.636686 \\ \mathrm{O} & 1.330309 & -4.419698 & -0.005528 \\ \mathrm{H} & 1.173785 & -5.355406 & 0.181719 \\ \mathrm{H} & 2.120009 & -4.357615 & -0.575343 \\ \mathrm{O} & -4.313706 & 0.578556 & 1.366879 \\ \mathrm{H} & -4.888852 & 0.724817 & 2.130356 \\ \mathrm{H} & -4.170898 & 1.464032 & 0.951771 \\ \mathrm{O} & -0.197719 & 3.475960 & -2.326806 \\ \mathrm{H} & -0.153362 & 4.130346 & -3.037047 \\ \mathrm{H} & 0.043813 & 3.930878 & -1.486938 \\ \mathrm{O} & 3.281638 & 1.190666 & -2.670134 \\ \mathrm{H} & 3.628034 & 1.830017 & -3.308371 \\ \mathrm{H} & 3.860189 & 1.227354 & -1.882233 \\ \mathrm{O} & 0.560231 & 4.415923 & 0.186356 \\ \mathrm{H} & 1.110380 & 5.208322 & 0.275863 \\ \mathrm{H} & -0.304599 & 4.626147 & 0.638771 \\ \mathrm{O} & -1.648391 & -2.908936 & -3.123407 \\ \mathrm{H} & -1.545974 & -3.699060 & -3.673959 \\ \mathrm{H} & -1.366382 & -2.139942 & -3.669231 \\ \mathrm{O} & 3.946849 & 1.539974 & 2.260848\end{array}$




$\begin{array}{crrr}\mathrm{H} & 4.346121 & 2.256732 & 2.776885 \\ \mathrm{H} & 3.789702 & 0.791550 & 2.878819 \\ \mathrm{O} & 3.175813 & -0.744716 & 3.709272 \\ \mathrm{H} & 3.760316 & -1.519245 & 3.730587 \\ \mathrm{H} & 2.849387 & -0.645342 & 4.618288 \\ \mathrm{O} & -1.833297 & 4.825769 & 1.342796 \\ \mathrm{H} & -2.211417 & 5.612206 & 1.759447 \\ \mathrm{H} & -2.574650 & 4.312325 & 0.941391 \\ \mathrm{O} & -0.861444 & -0.431659 & -4.129476 \\ \mathrm{H} & -0.145481 & 0.036615 & -3.642460 \\ \mathrm{H} & -0.921539 & -0.040314 & -5.013117 \\ \mathrm{O} & -2.928433 & -3.389738 & 2.576973 \\ \mathrm{H} & -2.796397 & -4.341134 & 2.445300 \\ \mathrm{H} & -3.870234 & -3.288203 & 2.785225 \\ \mathrm{O} & 4.660676 & 0.642366 & -0.266385 \\ \mathrm{H} & 5.570574 & 0.325163 & -0.363451 \\ \mathrm{H} & 4.599229 & 1.063826 & 0.620400 \\ \mathrm{O} & 3.363590 & -3.340266 & -1.650352 \\ \mathrm{H} & 3.268387 & -3.391582 & -2.614464 \\ \mathrm{H} & 4.302719 & -3.508902 & -1.476078 \\ \mathrm{O} & -3.438470 & 2.904018 & 0.175753 \\ \mathrm{H} & -2.533969 & 2.426122 & 0.197420 \\ \mathrm{H} & -3.677705 & 3.035287 & -0.753176 \\ \mathrm{O} & -1.958885 & -0.032917 & -1.532329 \\ \mathrm{H} & -1.858430 & 0.033517 & -2.498944 \\ \mathrm{H} & -2.715693 & -0.650416 & -1.372796 \\ \mathrm{O} & -3.765428 & -2.052989 & -1.416314 \\ \mathrm{H} & -4.717979 & -2.017660 & -1.583911 \\ \mathrm{H} & -3.356698 & -2.573957 & -2.133476 \\ \mathrm{O} & 2.221273 & -0.860163 & -0.320792 \\ \mathrm{H} & 2.430446 & -1.698716 & -0.770457 \\ \mathrm{H} & 3.054880 & -0.345868 & -0.323749 \\ \mathrm{H} & -0.953285 & 2.314475 & 1.407374 \\ \mathrm{O} & -1.173462 & 1.814023 & 0.609976\end{array}$

\begin{tabular}{|c|c|c|c|}
\hline \multicolumn{4}{|c|}{$\left[{ }^{1} \mathrm{Y}\left(\mathrm{H}_{2} \mathrm{O}\right)_{23}(\mathrm{OH})\right]^{2+}(\mathrm{d})$} \\
\hline $\mathrm{Y}$ & 0.272329 & 0.312652 & -0.462801 \\
\hline $\mathrm{O}$ & 2.035740 & 0.165798 & -2.034035 \\
\hline $\mathrm{O}$ & -1.848696 & 0.094760 & 236 \\
\hline $\mathrm{H}$ & 2.845939 & 0.723444 & -1.9 \\
\hline $\mathrm{H}$ & 2.378413 & -0.729556 & 510 \\
\hline $\mathrm{O}$ & -0.973127 & -1.28 & 753 \\
\hline $\mathrm{O}$ & -0.874064 & 1.502154 & 1.275991 \\
\hline $\mathrm{O}$ & 1.834495 & -1.060024 & 0.784465 \\
\hline $\mathrm{H}$ & -2.413976 & -0.659369 & -1.283032 \\
\hline
\end{tabular}




\begin{tabular}{|c|c|c|c|}
\hline $\mathrm{H}$ & -2.476705 & 0.816580 & -1.793287 \\
\hline & -1.170825 & -1.178647 & 1.905386 \\
\hline & -1.710939 & -1.799465 & 0.567725 \\
\hline & -0.636932 & 2.432592 & 1.540225 \\
\hline & -1.787451 & 1.333624 & 1.581578 \\
\hline & 2.807104 & -0.927625 & 0.762750 \\
\hline 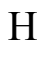 & 1.645402 & -1.925716 & 1.207158 \\
\hline & -1.964222 & -1.227050 & 3.561935 \\
\hline $\mathrm{H}$ & -1.572996 & -0.893372 & 4.382849 \\
\hline $\mathrm{H}$ & -2.671386 & -0.588758 & 3.312864 \\
\hline 0 & -3.319745 & -1.946080 & -0.574911 \\
\hline $\mathrm{H}$ & -4.162933 & -1.506766 & -0.337902 \\
\hline $\mathrm{H}$ & -3.475888 & -2.569031 & -1.304522 \\
\hline 0 & -0.070478 & 3.991007 & 1.957066 \\
\hline $\mathrm{H}$ & -0.644834 & 4.706895 & 2.262428 \\
\hline $\mathrm{H}$ & 0.430644 & 4.336150 & 1.186116 \\
\hline $\mathrm{O}$ & 4.378723 & 1.418270 & -1.449818 \\
\hline $\mathrm{H}$ & 5.136083 & 1.494255 & -2.047638 \\
\hline $\mathrm{H}$ & 4.648134 & 0.831475 & -0.715214 \\
\hline $\mathrm{O}$ & 3.303432 & -2.282802 & -2.323887 \\
\hline $\mathrm{H}$ & 3.773973 & -2.621441 & -3.098571 \\
\hline $\mathrm{H}$ & 2.867332 & -3.046204 & -1.897938 \\
\hline $\mathrm{O}$ & 4.580920 & -0.308909 & 0.865253 \\
\hline $\mathrm{H}$ & 5.325682 & -0.919789 & 0.972112 \\
\hline $\mathrm{H}$ & 4.589986 & 0.297326 & 1.636389 \\
\hline $\mathrm{O}$ & -4.060962 & 1.844999 & -1.713790 \\
\hline $\mathrm{H}$ & -4.557980 & 2.075702 & -2.512768 \\
\hline $\mathrm{H}$ & -3.506333 & 2.651242 & -1.476358 \\
\hline $\mathrm{O}$ & 1.339848 & -3.675013 & 1.773502 \\
\hline $\mathrm{H}$ & 1.964561 & -4.087096 & 2.389383 \\
\hline $\mathrm{H}$ & 0.442634 & -3.773499 & 2.192015 \\
\hline $\mathrm{O}$ & -1.091513 & -3.886979 & 2.960404 \\
\hline $\mathrm{H}$ & -1.482989 & -4.660880 & 3.389272 \\
\hline $\mathrm{H}$ & -1.568691 & -3.098897 & 3.285650 \\
\hline $\mathrm{O}$ & 3.972098 & 1.663073 & 2.779089 \\
\hline $\mathrm{H}$ & 3.802451 & 1.501585 & 3.720489 \\
\hline $\mathrm{H}$ & 4.459800 & 2.501796 & 2.744761 \\
\hline $\mathrm{O}$ & -2.274694 & 3.643587 & -1.101906 \\
\hline $\mathrm{H}$ & -1.396656 & 3.200975 & -1.295856 \\
\hline $\mathrm{H}$ & -2.219418 & 4.558938 & -1.408696 \\
\hline $\mathrm{O}$ & -3.493368 & 0.785671 & 2.371638 \\
\hline $\mathrm{H}$ & -4.129842 & 0.554846 & 1.650282 \\
\hline $\mathrm{H}$ & -3.933440 & 1.464761 & 2.905737 \\
\hline $\mathrm{O}$ & 1.548775 & -4.105734 & -0.942688 \\
\hline $\mathrm{H}$ & 1.405641 & -5.018694 & -1.232734 \\
\hline $\mathrm{H}$ & 1.500176 & -4.109139 & 0.044083 \\
\hline
\end{tabular}




$\begin{array}{rrrr}\mathrm{O} & -2.250053 & -2.888066 & -3.171544 \\ \mathrm{H} & -2.512897 & -2.344621 & -3.931153 \\ \mathrm{H} & -2.182109 & -3.791151 & -3.519370 \\ \mathrm{O} & 1.417321 & 4.171687 & -0.357179 \\ \mathrm{H} & 0.884586 & 3.572301 & -0.972043 \\ \mathrm{H} & 1.942177 & 4.786286 & -0.888769 \\ \mathrm{O} & 1.949625 & 1.711895 & 0.595885 \\ \mathrm{H} & 2.418937 & 1.637732 & 1.445044 \\ \mathrm{H} & 2.011707 & 2.653906 & 0.305486 \\ \mathrm{O} & 0.051927 & -1.845455 & -1.648095 \\ \mathrm{H} & -0.672508 & -2.095409 & -2.253722 \\ \mathrm{H} & 0.536956 & -2.671782 & -1.430889 \\ \mathrm{O} & -5.074027 & 0.147519 & 0.178827 \\ \mathrm{H} & -6.039045 & 0.165443 & 0.271776 \\ \mathrm{H} & -4.837967 & 0.828958 & -0.504047 \\ \mathrm{H} & 0.219998 & 2.239572 & -2.482912 \\ \mathrm{O} & 0.020246 & 2.305519 & -1.536312\end{array}$

$\left[{ }^{1} \mathrm{Y}\left(\mathrm{H}_{2} \mathrm{O}\right)_{23}(\mathrm{OH})\right]^{2+}(\mathrm{e})$

$\mathrm{E}=-1872.756479$

$\begin{array}{rrrr}\mathrm{Y} & 0.244154 & -0.028430 & -0.136543 \\ \mathrm{O} & -0.482930 & -0.663334 & -2.291532 \\ \mathrm{O} & -1.744209 & 0.153547 & 0.442165 \\ \mathrm{H} & -0.138502 & -1.411586 & -2.842476 \\ \mathrm{H} & -1.428038 & -0.561698 & -2.537658 \\ \mathrm{H} & -2.514402 & -0.066163 & 0.976814 \\ \mathrm{O} & 0.050000 & -2.904460 & -3.689894 \\ \mathrm{H} & 0.441795 & -3.096675 & -4.552755 \\ \mathrm{H} & 0.353951 & -3.593530 & -3.065776 \\ \mathrm{O} & -3.231679 & -0.464193 & -2.693503 \\ \mathrm{H} & -3.649083 & -0.770402 & -3.512746 \\ \mathrm{H} & -3.448627 & -1.128207 & -1.988864 \\ \mathrm{O} & -3.563048 & -2.208760 & -0.604454 \\ \mathrm{H} & -4.100718 & -3.008050 & -0.713930 \\ \mathrm{H} & -4.024297 & -1.644735 & 0.068198 \\ \mathrm{O} & -4.617757 & -0.455296 & 1.205944 \\ \mathrm{H} & -5.279131 & -0.631814 & 1.889845 \\ \mathrm{H} & -4.677564 & 0.503661 & 0.985114 \\ \mathrm{O} & -4.360464 & 2.242542 & 0.551428 \\ \mathrm{H} & -5.118237 & 2.847005 & 0.534982 \\ \mathrm{H} & -3.960194 & 2.267827 & -0.353485 \\ \mathrm{O} & -3.077880 & 2.178560 & -1.896695 \\ \mathrm{H} & -3.362049 & 2.758790 & -2.619620 \\ \mathrm{H} & -3.204204 & 1.251794 & -2.218829 \\ \mathrm{O} & 0.844200 & -4.151137 & -1.314371 \\ \mathrm{H} & 0.191882 & -3.830593 & -0.651094\end{array}$




\begin{tabular}{|c|c|c|c|}
\hline $\mathrm{H}$ & 1.188756 & -4.997549 & -0.995373 \\
\hline $\mathrm{O}$ & 2.299859 & 1.268822 & -0.444624 \\
\hline $\mathrm{H}$ & 2.592097 & 1.733273 & -1.252329 \\
\hline $\mathrm{H}$ & 3.098790 & 1.089723 & 0.099193 \\
\hline $\mathrm{O}$ & 4.226138 & 0.189341 & 1.210757 \\
\hline $\mathrm{H}$ & 4.743676 & 0.619927 & 1.906955 \\
\hline $\mathrm{H}$ & 3.477102 & -0.276658 & 1.640052 \\
\hline $\mathrm{O}$ & 1.684581 & -0.968116 & 1.755737 \\
\hline $\mathrm{H}$ & 1.335703 & -0.765580 & 2.652715 \\
\hline $\mathrm{H}$ & 1.827600 & -1.950869 & 1.755170 \\
\hline $\mathrm{O}$ & 1.728472 & -3.666483 & 2.119559 \\
\hline $\mathrm{H}$ & 2.407351 & -4.212408 & 2.541832 \\
\hline $\mathrm{H}$ & 0.929217 & -3.708080 & 2.681273 \\
\hline $\mathrm{O}$ & 0.113447 & -0.299297 & 3.927440 \\
\hline $\mathrm{H}$ & 0.265570 & -0.007935 & 4.838586 \\
\hline $\mathrm{H}$ & -0.001561 & 0.508696 & 3.368919 \\
\hline $\mathrm{O}$ & 0.257450 & 1.535432 & 1.881528 \\
\hline $\mathrm{H}$ & 0.957643 & 2.236706 & 1.945619 \\
\hline $\mathrm{H}$ & -0.605108 & 2.029197 & 1.845442 \\
\hline $\mathrm{O}$ & -1.982571 & 3.086130 & 1.924088 \\
\hline $\mathrm{H}$ & -2.221154 & 3.690191 & 2.640705 \\
\hline $\mathrm{H}$ & -2.822163 & 2.781670 & 1.519465 \\
\hline $\mathrm{O}$ & 2.124877 & 3.515711 & 1.825836 \\
\hline $\mathrm{H}$ & 2.270523 & 4.220100 & 2.473247 \\
\hline $\mathrm{H}$ & 1.943828 & 3.951358 & 0.966604 \\
\hline $\mathrm{O}$ & 1.340075 & 4.414037 & -0.773425 \\
\hline $\mathrm{H}$ & 1.065890 & 5.328976 & -0.938614 \\
\hline $\mathrm{H}$ & 1.991184 & 4.182792 & -1.468863 \\
\hline $\mathrm{O}$ & 3.024073 & 3.060072 & -2.611523 \\
\hline $\mathrm{H}$ & 2.646037 & 2.859886 & -3.482836 \\
\hline $\mathrm{H}$ & 3.963498 & 3.246018 & -2.770235 \\
\hline $\mathrm{O}$ & -0.778283 & -2.849088 & 3.104503 \\
\hline $\mathrm{H}$ & -1.480733 & -3.296955 & 3.599056 \\
\hline $\mathrm{H}$ & -0.650434 & -1.968512 & 3.522853 \\
\hline $\mathrm{O}$ & 1.892005 & -1.589723 & -1.019962 \\
\hline $\mathrm{H}$ & 1.731089 & -2.547266 & -1.160205 \\
\hline $\mathrm{H}$ & 2.843710 & -1.409335 & -1.213541 \\
\hline $\mathrm{O}$ & 4.545610 & -0.992182 & -1.315967 \\
\hline $\mathrm{H}$ & 5.269482 & -1.517658 & -1.684663 \\
\hline $\mathrm{H}$ & 4.817468 & -0.699686 & -0.425197 \\
\hline $\mathrm{O}$ & -0.260837 & 2.079137 & -1.237628 \\
\hline $\mathrm{H}$ & 0.128248 & 2.941702 & -0.986623 \\
\hline $\mathrm{H}$ & -1.217661 & 2.213756 & -1.409389 \\
\hline 0 & -0.733205 & -2.512053 & 0.262997 \\
\hline $\mathrm{H}$ & -1.652457 & -2.370096 & -0.056999 \\
\hline $\mathrm{H}$ & -0.831167 & -2.675911 & 1.227423 \\
\hline
\end{tabular}




\begin{tabular}{crrc}
\multicolumn{5}{c}{$\left.{ }^{1} \mathrm{Y}\left(\mathrm{H}_{2} \mathrm{O}\right)_{23}(\mathrm{OH})\right]^{2+}(\mathrm{f})$} \\
$\mathrm{E}=-1872.761853$ & & \\
$\mathrm{Y}$ & 0.292134 & 0.030410 & 0.339492 \\
$\mathrm{O}$ & 1.639155 & -2.058884 & 0.043046 \\
$\mathrm{O}$ & 2.002891 & 1.661339 & 0.810603 \\
$\mathrm{H}$ & 1.171463 & -2.913948 & 0.222350 \\
$\mathrm{H}$ & 1.908918 & -2.166458 & -0.909519 \\
$\mathrm{O}$ & -0.255788 & 1.896440 & -1.127326 \\
$\mathrm{O}$ & -1.007335 & -0.967607 & -1.371089 \\
$\mathrm{O}$ & -1.147009 & 1.227602 & 1.557233 \\
$\mathrm{H}$ & 2.156832 & 2.432882 & 0.223591 \\
$\mathrm{H}$ & 2.778496 & 1.549776 & 1.404097 \\
$\mathrm{H}$ & -1.027901 & 2.462715 & -0.880081 \\
$\mathrm{H}$ & 0.458688 & 2.477348 & -1.446950 \\
$\mathrm{H}$ & -1.346797 & -1.891652 & -1.390732 \\
$\mathrm{H}$ & -1.349679 & -0.533686 & -2.191232 \\
$\mathrm{H}$ & -0.744835 & 1.990527 & 2.000967 \\
$\mathrm{O}$ & -2.616101 & 2.794290 & -0.219163 \\
$\mathrm{H}$ & -2.360829 & 2.205486 & 0.532401 \\
$\mathrm{H}$ & -3.021513 & 3.606338 & 0.160307 \\
$\mathrm{O}$ & 2.193679 & 3.493077 & -1.269779 \\
$\mathrm{H}$ & 2.230613 & 4.461351 & -1.270020 \\
$\mathrm{H}$ & 2.935057 & 3.173077 & -1.825274 \\
$\mathrm{O}$ & 0.448091 & -4.486399 & -0.210630 \\
$\mathrm{H}$ & 0.463900 & -5.337226 & 0.249065 \\
$\mathrm{H}$ & -0.433001 & -4.391446 & -0.628426 \\
$\mathrm{O}$ & 2.145919 & -2.686235 & -2.572795 \\
$\mathrm{H}$ & 2.047304 & -3.636262 & -2.731883 \\
$\mathrm{H}$ & 1.619269 & -2.222843 & -3.250177 \\
$\mathrm{O}$ & -1.982642 & -3.604666 & -1.358856 \\
$\mathrm{H}$ & -2.401503 & -4.012189 & -2.131554 \\
$\mathrm{H}$ & -2.655011 & -3.598581 & -0.627855 \\
$\mathrm{O}$ & 4.145220 & 0.957163 & 2.481439 \\
$\mathrm{H}$ & 5.060027 & 1.275270 & 2.491491 \\
$\mathrm{H}$ & 4.179439 & -0.015541 & 2.334248 \\
$\mathrm{O}$ & -1.831892 & 0.086195 & -3.759114 \\
$\mathrm{H}$ & -2.465980 & -0.423495 & -4.285650 \\
$\mathrm{H}$ & -2.304939 & 0.936021 & -3.515605 \\
$\mathrm{O}$ & -3.131504 & 2.227111 & -2.897043 \\
$\mathrm{H}$ & -3.916714 & 2.654096 & -3.265512 \\
$\mathrm{H}$ & -3.118486 & 2.402979 & -1.929150 \\
$\mathrm{O}$ & -3.302477 & -3.344645 & 0.932708 \\
$\mathrm{H}$ & -3.957245 & -2.639596 & 1.217932 \\
$\mathrm{H}$ & -3.471261 & -4.117967 & 1.491204 \\
$\mathrm{O}$ & 3.645208 & -1.770487 & 2.006736
\end{tabular}




$\begin{array}{crrr}\mathrm{H} & 3.164792 & -2.015637 & 1.184339 \\ \mathrm{H} & 4.157072 & -2.544501 & 2.285038 \\ \mathrm{O} & -3.762301 & 5.091489 & 0.858433 \\ \mathrm{H} & -3.492520 & 6.010142 & 0.716193 \\ \mathrm{H} & -4.664209 & 5.127756 & 1.208772 \\ \mathrm{O} & 0.841809 & -0.572270 & -4.145242 \\ \mathrm{H} & 1.201357 & -0.383385 & -5.024523 \\ \mathrm{H} & -0.095520 & -0.264126 & -4.151952 \\ \mathrm{O} & 4.164072 & 1.928089 & -2.535139 \\ \mathrm{H} & 5.066376 & 1.915819 & -2.178338 \\ \mathrm{H} & 4.272276 & 1.908738 & -3.499391 \\ \mathrm{O} & -1.008127 & -1.734525 & 1.436513 \\ \mathrm{H} & -1.723037 & -2.348383 & 1.164422 \\ \mathrm{H} & -1.319776 & -1.388058 & 2.301032 \\ \mathrm{O} & -2.568068 & -0.242967 & 3.198173 \\ \mathrm{H} & -2.081312 & 0.451821 & 2.652015 \\ \mathrm{H} & -2.539348 & 0.029711 & 4.125944 \\ \mathrm{O} & 1.197795 & -0.447840 & 2.522789 \\ \mathrm{H} & 1.906476 & -1.109309 & 2.633326 \\ \mathrm{H} & 1.339362 & 0.226502 & 3.235862 \\ \mathrm{O} & 1.978797 & 1.413864 & 4.295216 \\ \mathrm{H} & 1.950294 & 1.480118 & 5.260220 \\ \mathrm{H} & 2.914881 & 1.448206 & 4.024083 \\ \mathrm{O} & 1.843697 & 0.183346 & -1.656850 \\ \mathrm{H} & 2.712210 & 0.607972 & -1.761699 \\ \mathrm{H} & 1.529285 & -0.025620 & -2.566090 \\ \mathrm{O} & -4.689642 & -1.296305 & 1.800134 \\ \mathrm{H} & -5.593306 & -1.194842 & 2.128129 \\ \mathrm{H} & -4.080461 & -0.823280 & 2.414449\end{array}$

$\begin{array}{lrrr}{\left[{ }^{1} \mathrm{Y}\left(\mathrm{H}_{2} \mathrm{O}\right)_{23}(\mathrm{OH})\right]^{2+}(\mathrm{g})} \\ \mathrm{E}=-1872.772272 \\ \mathrm{Y} & -0.164096 & -0.114525 & 0.072740 \\ \mathrm{O} & -0.644018 & 1.196982 & -1.999175 \\ \mathrm{O} & 1.371069 & -0.603714 & 1.781202 \\ \mathrm{H} & -1.268508 & 1.962530 & -1.856783 \\ \mathrm{H} & 0.152873 & 1.625009 & -2.415582 \\ \mathrm{O} & -1.420017 & -1.181976 & 1.780267 \\ \mathrm{O} & 0.998421 & 2.026353 & 0.519202 \\ \mathrm{O} & 0.092391 & -2.248726 & -0.580842 \\ \mathrm{H} & 1.245402 & -1.377427 & 2.397198 \\ \mathrm{H} & 2.338356 & -0.425613 & 1.781477 \\ \mathrm{H} & -2.132106 & -0.839342 & 2.373617 \\ \mathrm{H} & -1.367094 & -2.164201 & 1.855953 \\ \mathrm{H} & 0.584885 & 2.904903 & 0.383814 \\ \mathrm{H} & 1.950948 & 2.177997 & 0.700702\end{array}$




\begin{tabular}{|c|c|c|c|}
\hline $\mathrm{H}$ & -0.360546 & -2.573875 & -1.379672 \\
\hline D & 1.257195 & -2.854267 & 3.222534 \\
\hline $\mathrm{H}$ & 1.186656 & -3.026223 & 4.171444 \\
\hline & 0.625334 & -3.451480 & 2.767791 \\
\hline & -3.494630 & -0.098158 & 3.174418 \\
\hline $\mathrm{H}$ & -4.007425 & -0.482885 & 3.899412 \\
\hline - & -4.123424 & 0.154241 & 2.470873 \\
\hline ) & -2.197223 & 3.361747 & -1.545873 \\
\hline & -2.476181 & 3.987858 & -2.229532 \\
\hline & -1.683761 & 3.874581 & -0.886616 \\
\hline & 1.348481 & 2.663998 & -3.092859 \\
\hline & 1.395836 & 2.991608 & -4.001880 \\
\hline & 2.270147 & 2.532101 & -2.785920 \\
\hline & -0.440361 & 4.496425 & 0.414307 \\
\hline$\exists$ & -0.048534 & 5.372838 & 0.279241 \\
\hline & -0.801170 & 4.480888 & 1.326042 \\
\hline$J$ & -1.432159 & -3.213048 & -3.029909 \\
\hline H & -1.171214 & -3.857273 & -3.704177 \\
\hline 7 & -1.625660 & -2.370736 & -3.492890 \\
\hline & 3.815005 & 2.310986 & 0.806215 \\
\hline $\mathrm{H}$ & 4.287035 & 3.028439 & 1.255123 \\
\hline & 4.084903 & 1.461075 & 1.247364 \\
\hline 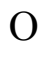 & 4.139676 & -0.143993 & 1.862911 \\
\hline & 4.504707 & -0.869521 & 1.269915 \\
\hline $\mathrm{H}$ & 4.512418 & -0.294800 & 2.744878 \\
\hline D & -1.426818 & 3.691206 & 2.937898 \\
\hline$H$ & -0.819432 & 3.608841 & 3.690387 \\
\hline 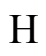 & -2.272272 & 3.977825 & 3.319485 \\
\hline $\mathrm{O}$ & -2.097940 & -0.543904 & -3.632627 \\
\hline $\mathrm{H}$ & -1.460630 & 0.148911 & -3.347675 \\
\hline & -2.579109 & -0.203623 & -4.401624 \\
\hline $\mathrm{O}$ & -0.676683 & -3.762492 & 1.482391 \\
\hline $\mathrm{H}$ & -0.376536 & -3.354645 & 0.617273 \\
\hline $\mathrm{H}$ & -1.272439 & -4.493221 & 1.263622 \\
\hline $\mathrm{O}$ & 3.733157 & 1.848302 & -1.886798 \\
\hline $\mathrm{H}$ & 4.569077 & 1.664590 & -2.341257 \\
\hline $\mathrm{H}$ & 3.955623 & 2.145459 & -0.972457 \\
\hline $\mathrm{O}$ & -4.324457 & 0.810374 & 0.649691 \\
\hline $\mathrm{H}$ & -3.954072 & 0.200326 & -0.026536 \\
\hline $\mathrm{H}$ & -5.070724 & 1.279221 & 0.248138 \\
\hline $\mathrm{O}$ & -1.694543 & 1.572367 & 0.944449 \\
\hline $\mathrm{H}$ & -1.538668 & 2.124093 & 1.734778 \\
\hline $\mathrm{H}$ & -2.671210 & 1.479393 & 0.856596 \\
\hline$U$ & -2.540836 & -0.702058 & -0.863206 \\
\hline $\mathrm{H}$ & -2.618583 & -0.611868 & -1.838054 \\
\hline $\mathrm{H}$ & -2.758238 & -1.657705 & -0.688310 \\
\hline
\end{tabular}




$\begin{array}{lrrr}\mathrm{O} & -3.076213 & -3.375466 & -0.797674 \\ \mathrm{H} & -3.955538 & -3.780486 & -0.812972 \\ \mathrm{H} & -2.645693 & -3.584604 & -1.654511 \\ \mathrm{O} & 1.867019 & -0.136655 & -1.246586 \\ \mathrm{H} & 2.248906 & -1.025663 & -1.430994 \\ \mathrm{H} & 2.539386 & 0.530991 & -1.504186 \\ \mathrm{O} & 2.600279 & -2.772907 & -1.203757 \\ \mathrm{H} & 1.640206 & -2.759219 & -0.893149 \\ \mathrm{H} & 2.668026 & -3.423886 & -1.916919 \\ \mathrm{O} & 4.874819 & -2.024119 & 0.181352 \\ \mathrm{H} & 5.544239 & -2.708022 & 0.323241 \\ \mathrm{H} & 4.100624 & -2.448993 & -0.253772\end{array}$

$\left[{ }^{1} \mathrm{Y}\left(\mathrm{H}_{2} \mathrm{O}\right)_{23}(\mathrm{OH})\right]^{2+}(\mathrm{h})$

$\mathrm{E}=-1872.771379$

$\begin{array}{rrrr}\mathrm{Y} & -0.004904 & 0.004220 & -0.145231 \\ \mathrm{O} & -1.904655 & 0.983707 & 0.808219 \\ \mathrm{O} & -1.711903 & -0.944493 & -1.565733 \\ \mathrm{H} & -2.212321 & 1.910923 & 0.499502 \\ \mathrm{H} & -2.265147 & 0.862670 & 1.710342 \\ \mathrm{O} & 0.832219 & -2.128585 & -0.933482 \\ \mathrm{O} & -0.148326 & 1.528430 & -1.683807 \\ \mathrm{H} & -1.626823 & -1.761407 & -2.113692 \\ \mathrm{H} & -2.652022 & -0.665180 & -1.604787 \\ \mathrm{H} & 0.390987 & -2.984726 & -0.724776 \\ \mathrm{H} & 1.359499 & -2.271347 & -1.743306 \\ \mathrm{H} & -0.946125 & 1.533952 & -2.237324 \\ \mathrm{O} & -2.694779 & 3.297068 & 0.038108 \\ \mathrm{H} & -2.931166 & 3.179171 & -0.907155 \\ \mathrm{H} & -1.923710 & 3.899222 & 0.071991 \\ \mathrm{O} & -4.391561 & -0.147582 & -1.383226 \\ \mathrm{H} & -5.149094 & -0.551758 & -1.832431 \\ \mathrm{H} & -4.491813 & -0.356575 & -0.427967 \\ \mathrm{O} & -4.253869 & -1.206932 & 1.262538 \\ \mathrm{H} & -4.945170 & -1.836159 & 1.520706 \\ \mathrm{H} & -4.090943 & -0.638881 & 2.049018 \\ \mathrm{O} & -1.469294 & -3.365072 & -2.837200 \\ \mathrm{H} & -2.009362 & -3.762739 & -3.534657 \\ \mathrm{H} & -1.432401 & -3.997864 & -2.094670 \\ \mathrm{O} & -0.787607 & -4.247881 & -0.261730 \\ \mathrm{H} & -0.529485 & -5.057433 & 0.202975 \\ \mathrm{H} & -1.167750 & -3.630828 & 0.402808 \\ \mathrm{O} & -3.240925 & 2.250743 & -2.475806 \\ \mathrm{H} & -3.708087 & 2.661931 & -3.217743 \\ \mathrm{H} & -3.801215 & 1.510157 & -2.167297 \\ \mathrm{O} & -0.145771 & 4.460708 & 0.314156\end{array}$




\begin{tabular}{|c|c|c|c|}
\hline $\mathrm{H}$ & 0.008914 & 5.337402 & \\
\hline $\mathrm{H}$ & 0.341110 & 4.413575 & -0.546945 \\
\hline $\mathrm{O}$ & -3.072630 & 0.313597 & 3.286833 \\
\hline $\mathrm{H}$ & -3.427110 & 0.962759 & 3.913228 \\
\hline $\mathrm{H}$ & -2.350436 & -0.175571 & 3.739395 \\
\hline $\mathrm{O}$ & -0.874182 & -1.314273 & 9333 \\
\hline $\mathrm{H}$ & -0.662292 & -1.813024 & 4.612555 \\
\hline $\mathrm{H}$ & -0.022579 & -1.043410 & \\
\hline $\mathrm{O}$ & 1.153667 & -0.860783 & 1.960679 \\
\hline $\mathrm{H}$ & 1.563915 & -1.758209 & \\
\hline $\mathrm{H}$ & 1.9237 & -0.314395 & 2.2 \\
\hline $\mathrm{O}$ & 3.450 & 0.34 & 2.7 \\
\hline $\mathrm{H}$ & 3.854 & & \\
\hline $\mathrm{H}$ & 3.672 & 1.209968 & 2.3 \\
\hline $\mathrm{O}$ & 2.44 & -3.208238 & \\
\hline $\mathrm{H}$ & 2.95 & -3.6 & \\
\hline $\mathrm{H}$ & & & \\
\hline $\mathrm{O}$ & 3.52 & 2.77 & \\
\hline $\mathrm{H}$ & 3.80 & 3.60 & 1.7 \\
\hline $\mathrm{H}$ & 3.70 & 2.8 & \\
\hline $\mathrm{O}$ & 4.06 & -2.1 & -0. \\
\hline $\mathrm{H}$ & 5.03 & -2.0 & -0.5 \\
\hline $\mathrm{H}$ & 3.85 & -2.46 & 020 \\
\hline $\mathrm{O}$ & & 24 & \\
\hline $\mathrm{H}$ & 2.84 & -2.20 & -3.7 \\
\hline $\mathrm{H}$ & 2.67 & -3.68 & -3.3 \\
\hline $\mathrm{O}$ & -1.46 & -1.9 & 1.1 \\
\hline $\mathrm{H}$ & -1.34 & -1.94 & \\
\hline $\mathrm{H}$ & -2.4 & -1.7 & 1. \\
\hline $\mathrm{O}$ & 2.3 & 0.16 & -0.7 \\
\hline $\mathrm{H}$ & 3.022212 & -0.528887 & -0.5 \\
\hline $\mathrm{H}$ & 2.802004 & 0.97 & -1.0 \\
\hline $\mathrm{O}$ & 0.808745 & 1.93 & 116 \\
\hline $\mathrm{H}$ & 1.74 & 2.18 & \\
\hline $\mathrm{H}$ & 0.35 & 2.79 & \\
\hline $\mathrm{H}$ & 0.58 & 2.78 & -1.9 \\
\hline $\mathrm{O}$ & 1.14 & 3.67 & -1.9 \\
\hline $\mathrm{H}$ & 0.983678 & 4.086520 & -2.814066 \\
\hline $\mathrm{H}$ & 2.691560 & & -1.6 \\
\hline $\mathrm{O}$ & 3.503500 & 2.636950 & -1.336006 \\
\hline $\mathrm{H}$ & 4.224176 & 2.822199 & -1.956244 \\
\hline \multicolumn{4}{|c|}{$\left[{ }^{1} \mathrm{Y}\left(\mathrm{H}_{2} \mathrm{O}\right)_{8}\right]^{3+}$} \\
\hline \multicolumn{4}{|c|}{$E=-649.1800494$} \\
\hline $\mathrm{O}$ & 0.000000 & 2.047598 & 1.298952 \\
\hline $\mathrm{H}$ & 0.558982 & 2.836354 & 1.171778 \\
\hline
\end{tabular}




\begin{tabular}{|c|c|c|c|}
\hline $\mathrm{H}$ & -0.573139 & 2.251675 & \\
\hline $\mathrm{O}$ & -2.047598 & 0.000000 & \\
\hline $\mathrm{H}$ & -2.836354 & 0.558982 & \\
\hline $\mathrm{H}$ & -2.251675 & -0.573139 & \\
\hline $\mathrm{O}$ & 0.000000 & -2.047598 & \\
\hline $\mathrm{H}$ & -0.558982 & -2.836354 & \\
\hline $\mathrm{H}$ & 0.573139 & -2.251675 & \\
\hline $\mathrm{O}$ & 2.04 & 0.000000 & \\
\hline $\mathrm{H}$ & 2.836 & -0.558982 & \\
\hline $\mathrm{H}$ & 2.25 & 0.5731 & \\
\hline $\mathrm{O}$ & 1.44 & & \\
\hline $\mathrm{H}$ & 2.40 & 1.6 & \\
\hline $\mathrm{H}$ & 1.18 & & \\
\hline $\mathrm{O}$ & -1.4 & 1.4 & -1.2 \\
\hline $\mathrm{H}$ & -1.6 & & \\
\hline $\mathrm{H}$ & -1.9 & 1.18 & \\
\hline $\mathrm{O}$ & -1.4 & & \\
\hline $\mathrm{H}$ & -2.4 & & -1. \\
\hline $\mathrm{H}$ & -1.1 & -1.9 & -2. \\
\hline $\mathrm{O}$ & 1.4 & & -1.2 \\
\hline $\mathrm{H}$ & 1.6 & -2.4 & \\
\hline $\mathrm{H}$ & 1.9 & -1.18 & \\
\hline Y & 0.00 & 0.00 & \\
\hline \multicolumn{4}{|c|}{$\left[{ }^{1} \mathrm{Y}\left(\mathrm{H}_{2} \mathrm{O}\right)_{7}(\mathrm{OH})\right]^{2+}(\mathrm{a})$} \\
\hline \multicolumn{4}{|c|}{$E=-649.0550005$} \\
\hline $\mathrm{O}$ & & & -0.6 \\
\hline $\mathrm{H}$ & 2.3 & -1.9 & $-0 .($ \\
\hline $\mathrm{H}$ & 1.3 & -2.6 & -0.5 \\
\hline $\mathrm{O}$ & -1.3 & -2.0 & -0.2 \\
\hline $\mathrm{H}$ & -1.3 & -2.7 & \\
\hline $\mathrm{H}$ & -1.91 & -2.35 & -1.0 \\
\hline $\mathrm{O}$ & -1.4 & 0.51 & -1.8 \\
\hline $\mathrm{H}$ & -2.3 & 0.65 & -1. \\
\hline $\mathrm{H}$ & -1.2 & & -2.7 \\
\hline $\mathrm{O}$ & 1.4 & 0.6 & -1.8 \\
\hline $\mathrm{H}$ & 1.6 & & -2.2 \\
\hline $\mathrm{H}$ & 2.067166 & 0.066019 & -2.2 \\
\hline $\mathrm{O}$ & 2.13 & 0.668442 & \\
\hline $\mathrm{H}$ & 2.970397 & 1.114454 & 0.89 \\
\hline $\mathrm{H}$ & 2.200254 & 0.339255 & 2.0 \\
\hline $\mathrm{O}$ & -0.09 & & \\
\hline $\mathrm{H}$ & -0.587150 & 3.075231 & -0.16 \\
\hline $\mathrm{H}$ & & & \\
\hline $\mathrm{O}$ & -2.349524 & 0.578355 & 0.781805 \\
\hline $\mathrm{H}$ & -2.821888 & -0.102817 & 1.289360 \\
\hline
\end{tabular}




\begin{tabular}{|c|c|c|c|}
\hline $\mathrm{H}$ & -2.531143 & 1.411158 & \\
\hline Y & -0.001854 & -0.047934 & 0.128625 \\
\hline $\mathrm{O}$ & 0.022870 & -0.847789 & 1.990091 \\
\hline $\mathrm{H}$ & -0.089872 & -1.204101 & 2.878025 \\
\hline \multicolumn{4}{|c|}{$\left[{ }^{1} \mathrm{Y}\left(\mathrm{H}_{2} \mathrm{O}\right)_{7}(\mathrm{OH})\right]^{2+}(\mathrm{b})$} \\
\hline \multicolumn{4}{|c|}{$E=-649.0564975$} \\
\hline $\mathrm{O}$ & -2.172823 & -0.000163 & -1.237958 \\
\hline $\mathrm{H}$ & -3.124 & -0.0 & -1.0 \\
\hline $\mathrm{H}$ & -2.062089 & 0.000831 & -2.20 \\
\hline $\mathrm{O}$ & -0.841878 & -2.2679 & \\
\hline $\mathrm{H}$ & -0.237 & -3.026 & \\
\hline $\mathrm{H}$ & -1.620 & -2.575 & -0.3 \\
\hline $\mathrm{O}$ & 1.926617 & -1.6 & -0.1 \\
\hline $\mathrm{H}$ & 2.235 & -1.6 & -1.1 \\
\hline $\mathrm{H}$ & 2.608689 & -2.0 & \\
\hline $\mathrm{O}$ & -1.714 & & \\
\hline $\mathrm{H}$ & -2.184 & -0.7 & \\
\hline $\mathrm{H}$ & -2.185 & 0.7 & 2.0 \\
\hline $\mathrm{O}$ & -0.842 & 2.2 & \\
\hline $\mathrm{H}$ & -1.622 & 2.5 & -0.3 \\
\hline $\mathrm{H}$ & -0.238 & 3.0 & \\
\hline $\mathrm{O}$ & 1.926 & 1.6 & -0.1 \\
\hline $\mathrm{H}$ & & & \\
\hline $\mathrm{H}$ & 2.605 & 2.0 & \\
\hline $\mathrm{O}$ & 1.040 & -0.0 & \\
\hline $\mathrm{H}$ & 1.94 & -0.0 & \\
\hline $\mathrm{H}$ & 0.442 & -0.0 & \\
\hline Y & 0.04 & 0.0 & -0 . \\
\hline $\mathrm{O}$ & 0.513 & 0.0 & $-2 .($ \\
\hline $\mathrm{H}$ & 0.686475 & 0.00 & -3.0 \\
\hline \multicolumn{4}{|c|}{$\left[{ }^{1} \mathrm{Y}\left(\mathrm{H}_{2} \mathrm{O}\right)_{7}(\mathrm{OH})\right]^{2+}(\mathrm{c})$} \\
\hline \multicolumn{4}{|c|}{$E=-649.0550005$} \\
\hline $\mathrm{O}$ & 1.423858 & & \\
\hline $\mathrm{H}$ & 1.613 & 1.5 & -2.2 \\
\hline $\mathrm{H}$ & 2.067166 & 0.0 & -2.2 \\
\hline $\mathrm{O}$ & 1.601210 & -1.794621 & -0.6 \\
\hline $\mathrm{H}$ & 2.378990 & -1.9 & $-0 .($ \\
\hline $\mathrm{H}$ & 1.311743 & -2.657775 & -0.98 \\
\hline $\mathrm{O}$ & -1.303748 & -2.076392 & -0.3 \\
\hline $\mathrm{H}$ & -1.363801 & -2.736257 & 0.356290 \\
\hline $\mathrm{H}$ & -1.910452 & -2.354239 & -1.059759 \\
\hline $\mathrm{O}$ & -1.438701 & 0.516984 & -1.824133 \\
\hline $\mathrm{H}$ & -2.396716 & 0.650501 & -1.732373 \\
\hline $\mathrm{H}$ & -1.218875 & 0.610918 & -2.764699 \\
\hline
\end{tabular}




$\begin{array}{rrrr}\mathrm{O} & -0.093596 & 2.408600 & 0.340267 \\ \mathrm{H} & -0.587150 & 3.075231 & -0.164121 \\ \mathrm{H} & 0.449999 & 2.892598 & 0.984027 \\ \mathrm{O} & 2.137592 & 0.668442 & 1.116951 \\ \mathrm{H} & 2.970397 & 1.114454 & 0.896902 \\ \mathrm{H} & 2.200254 & 0.339255 & 2.030342 \\ \mathrm{O} & -2.349524 & 0.578355 & 0.781805 \\ \mathrm{H} & -2.821888 & -0.102817 & 1.289360 \\ \mathrm{H} & -2.531143 & 1.411158 & 1.247836 \\ \mathrm{Y} & -0.001854 & -0.047934 & 0.128625 \\ \mathrm{O} & 0.022870 & -0.847789 & 1.990091 \\ \mathrm{H} & -0.089872 & -1.204101 & 2.878025\end{array}$

$\left[{ }^{1} \mathrm{Y}\left(\mathrm{H}_{2} \mathrm{O}\right)_{7}(\mathrm{OH})\right]^{2+}(\mathrm{d})$

$\mathrm{E}=-649.0564975$

$\begin{array}{rrrr}\mathrm{O} & -0.841878 & -2.267903 & 0.110105 \\ \mathrm{H} & -0.237322 & -3.026549 & 0.167853 \\ \mathrm{H} & -1.620424 & -2.575565 & -0.382146 \\ \mathrm{O} & -2.172823 & -0.000163 & -1.237958 \\ \mathrm{H} & -3.124882 & -0.000604 & -1.051879 \\ \mathrm{H} & -2.062089 & 0.000831 & -2.204294 \\ \mathrm{O} & 1.926617 & -1.615677 & -0.198751 \\ \mathrm{H} & 2.235916 & -1.696716 & -1.117454 \\ \mathrm{H} & 2.608689 & -2.008380 & 0.368173 \\ \mathrm{O} & 1.040394 & -0.003984 & 2.157338 \\ \mathrm{H} & 1.943952 & -0.006238 & 2.510648 \\ \mathrm{H} & 0.442916 & -0.004558 & 2.923319 \\ \mathrm{O} & -1.714182 & -0.001335 & 1.752108 \\ \mathrm{H} & -2.184602 & -0.781564 & 2.089180 \\ \mathrm{H} & -2.185233 & 0.777382 & 2.091769 \\ \mathrm{O} & -0.842703 & 2.267928 & 0.112475 \\ \mathrm{H} & -1.622691 & 2.576232 & -0.377072 \\ \mathrm{H} & -0.238567 & 3.026870 & 0.170641 \\ \mathrm{O} & 1.926356 & 1.616882 & -0.191474 \\ \mathrm{H} & 2.239242 & 1.701976 & -1.108560 \\ \mathrm{H} & 2.605346 & 2.008878 & 0.379641 \\ \mathrm{Y} & 0.046902 & 0.000374 & -0.119851 \\ \mathrm{O} & 0.513730 & 0.002502 & -2.096488 \\ \mathrm{H} & 0.686475 & 0.007411 & -3.044478\end{array}$

$\left[{ }^{1} \mathrm{Y}\left(\mathrm{H}_{2} \mathrm{O}\right)_{7}(\mathrm{OH})\right]^{2+}(\mathrm{e})$

$\mathrm{E}=-649.0550005$
$\begin{array}{lllll}\mathrm{O} & -1.438701 & 0.516984 & -1.824133\end{array}$
$\begin{array}{lllll}\mathrm{H} & -2.396716 & 0.650501 & -1.732373\end{array}$

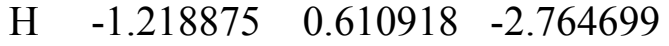
$\begin{array}{llll}\mathrm{O} & 1.423858 & 0.694220 & -1.832844\end{array}$ 


$\begin{array}{crrr}\mathrm{H} & 1.613948 & 1.557491 & -2.232835 \\ \mathrm{H} & 2.067166 & 0.066019 & -2.200926 \\ \mathrm{O} & 1.601210 & -1.794621 & -0.649737 \\ \mathrm{H} & 2.378990 & -1.975398 & -0.095458 \\ \mathrm{H} & 1.311743 & -2.657775 & -0.988861 \\ \mathrm{O} & -1.303748 & -2.076392 & -0.354915 \\ \mathrm{H} & -1.363801 & -2.736257 & 0.356290 \\ \mathrm{H} & -1.910452 & -2.354239 & -1.059759 \\ \mathrm{O} & -2.349524 & 0.578355 & 0.781805 \\ \mathrm{H} & -2.821888 & -0.102817 & 1.289360 \\ \mathrm{H} & -2.531143 & 1.411158 & 1.247836 \\ \mathrm{O} & -0.093596 & 2.408600 & 0.340267 \\ \mathrm{H} & -0.587150 & 3.075231 & -0.164121 \\ \mathrm{H} & 0.449999 & 2.892598 & 0.984027 \\ \mathrm{O} & 2.137592 & 0.668442 & 1.116951 \\ \mathrm{H} & 2.970397 & 1.114454 & 0.896902 \\ \mathrm{H} & 2.200254 & 0.339255 & 2.030342 \\ \mathrm{Y} & -0.001854 & -0.047934 & 0.128625 \\ \mathrm{O} & 0.022870 & -0.847789 & 1.990091 \\ \mathrm{H} & -0.089872 & -1.204101 & 2.878025\end{array}$

\begin{tabular}{crrr}
\multicolumn{5}{l}{$\left[{ }^{1} \mathrm{Y}\left(\mathrm{H}_{2} \mathrm{O}\right)_{7}(\mathrm{OH})\right]^{2+}(\mathrm{f})$} \\
$\mathrm{E}=-649.0564975$ \\
$\mathrm{O}$ & 1.926617 & -1.615677 & -0.198751 \\
$\mathrm{H}$ & 2.235916 & -1.696716 & -1.117454 \\
$\mathrm{H}$ & 2.608689 & -2.008380 & 0.368173 \\
$\mathrm{O}$ & -0.841878 & -2.267903 & 0.110105 \\
$\mathrm{H}$ & -0.237322 & -3.026549 & 0.167853 \\
$\mathrm{H}$ & -1.620424 & -2.575565 & -0.382146 \\
$\mathrm{O}$ & -2.172823 & -0.000163 & -1.237958 \\
$\mathrm{H}$ & -3.124882 & -0.000604 & -1.051879 \\
$\mathrm{H}$ & -2.062089 & 0.000831 & -2.204294 \\
$\mathrm{O}$ & 1.926356 & 1.616882 & -0.191474 \\
$\mathrm{H}$ & 2.239242 & 1.701976 & -1.108560 \\
$\mathrm{H}$ & 2.605346 & 2.008878 & 0.379641 \\
$\mathrm{O}$ & 1.040394 & -0.003984 & 2.157338 \\
$\mathrm{H}$ & 1.943952 & -0.006238 & 2.510648 \\
$\mathrm{H}$ & 0.442916 & -0.004558 & 2.923319 \\
$\mathrm{O}$ & -1.714182 & -0.001335 & 1.752108 \\
$\mathrm{H}$ & -2.184602 & -0.781564 & 2.089180 \\
$\mathrm{H}$ & -2.185233 & 0.777382 & 2.091769 \\
$\mathrm{O}$ & -0.842703 & 2.267928 & 0.112475 \\
$\mathrm{H}$ & -1.622691 & 2.576232 & -0.377072 \\
$\mathrm{H}$ & -0.238567 & 3.026870 & 0.170641 \\
$\mathrm{Y}$ & 0.046902 & 0.000374 & -0.119851 \\
$\mathrm{O}$ & 0.513730 & 0.002502 & -2.096488
\end{tabular}




\begin{tabular}{|c|c|c|c|}
\hline $\mathrm{H}$ & 0.686475 & 0.007411 & -3.044478 \\
\hline \multicolumn{4}{|c|}{$\left[{ }^{1} \mathrm{Y}\left(\mathrm{H}_{2} \mathrm{O}\right)_{7}(\mathrm{OH})\right]^{2+}(\mathrm{g})$} \\
\hline \multicolumn{4}{|c|}{$E=-649.0550005$} \\
\hline $\mathrm{O}$ & -1.303748 & -2.076392 & -0.354915 \\
\hline $\mathrm{H}$ & -1.363801 & -2.736257 & 0.356290 \\
\hline $\mathrm{H}$ & -1.910452 & -2.354239 & -1.059759 \\
\hline $\mathrm{O}$ & -1.438701 & 0.516984 & -1.824133 \\
\hline $\mathrm{H}$ & -2.396716 & 0.650 & -1.7 \\
\hline $\mathrm{H}$ & -1.218875 & 0.61 & -2.7 \\
\hline $\mathrm{O}$ & 1.423 & 0.69 & \\
\hline $\mathrm{H}$ & 1.613948 & 1.557491 & -2.2 \\
\hline $\mathrm{H}$ & 2.067166 & 0.066 & -2.2 \\
\hline $\mathrm{O}$ & 1.601210 & -1.794621 & -0. \\
\hline $\mathrm{H}$ & 2.378990 & -1.97 & -0. \\
\hline $\mathrm{H}$ & 1.311743 & -2.65 & -0 \\
\hline $\mathrm{O}$ & -2.349524 & 0.578355 & \\
\hline $\mathrm{H}$ & -2.821888 & -0.10 & 1.2 \\
\hline $\mathrm{H}$ & -2.531143 & 1.411158 & \\
\hline $\mathrm{O}$ & -0.093596 & 2.408600 & 0.3 \\
\hline $\mathrm{H}$ & -0.587150 & 3.075231 & -0.1 \\
\hline $\mathrm{H}$ & 0.449999 & 2.892598 & 0.984027 \\
\hline $\mathrm{O}$ & 2.137592 & 0.668442 & 1.1 \\
\hline $\mathrm{H}$ & 2.970397 & 1.114454 & 0.896902 \\
\hline $\mathrm{H}$ & 2.200254 & 0.339255 & 2.030342 \\
\hline Y & -0.001854 & -0.047934 & \\
\hline $\mathrm{O}$ & 0.022870 & -0.847789 & 1.990091 \\
\hline $\mathrm{H}$ & -0.089872 & -1.204101 & 2.878025 \\
\hline
\end{tabular}

$\left[{ }^{1} \mathrm{Y}\left(\mathrm{H}_{2} \mathrm{O}\right)_{7}(\mathrm{OH})\right]^{2+}(\mathrm{h})$

$\mathrm{E}=-649.0550005$

$\begin{array}{rrrr}\mathrm{O} & 2.349524 & 0.578356 & 0.781805 \\ \mathrm{H} & 2.821888 & -0.102817 & 1.289360 \\ \mathrm{H} & 2.531143 & 1.411158 & 1.247836 \\ \mathrm{O} & 0.093596 & 2.408600 & 0.340268 \\ \mathrm{H} & 0.587150 & 3.075231 & -0.164121 \\ \mathrm{H} & -0.449999 & 2.892598 & 0.984027 \\ \mathrm{O} & -2.137592 & 0.668442 & 1.116951 \\ \mathrm{H} & -2.970397 & 1.114454 & 0.896902 \\ \mathrm{H} & -2.200254 & 0.339255 & 2.030342 \\ \mathrm{O} & 1.303748 & -2.076392 & -0.354915 \\ \mathrm{H} & 1.363801 & -2.736257 & 0.356290 \\ \mathrm{H} & 1.910452 & -2.354239 & -1.059759 \\ \mathrm{O} & 1.438701 & 0.516984 & -1.824133 \\ \mathrm{H} & 2.396715 & 0.650501 & -1.732373 \\ \mathrm{H} & 1.218875 & 0.610918 & -2.764699\end{array}$




$\begin{array}{lrrr}\mathrm{O} & -1.423858 & 0.694220 & -1.832844 \\ \mathrm{H} & -1.613948 & 1.557491 & -2.232835 \\ \mathrm{H} & -2.067166 & 0.066019 & -2.200926 \\ \mathrm{O} & -1.601210 & -1.794621 & -0.649737 \\ \mathrm{H} & -2.378990 & -1.975398 & -0.095458 \\ \mathrm{H} & -1.311743 & -2.657775 & -0.988861 \\ \mathrm{Y} & 0.001854 & -0.047934 & 0.128625 \\ \mathrm{O} & -0.022870 & -0.847789 & 1.990091 \\ \mathrm{H} & 0.089872 & -1.204101 & 2.878025\end{array}$

\begin{tabular}{|c|c|c|c|}
\hline \multicolumn{4}{|c|}{$\begin{array}{l}{\left[{ }^{1} \mathrm{La}\left(\mathrm{H}_{2} \mathrm{O}\right)_{27}\right]^{3+}} \\
\mathrm{E}=-2499.877838\end{array}$} \\
\hline LA & 0.456551 & -0.173702 & -0.1 \\
\hline $\mathrm{O}$ & -0.345556 & -2.623177 & \\
\hline $\mathrm{H}$ & -0.888787 & -2.838715 & \\
\hline $\mathrm{H}$ & -0.533434 & -3.327672 & 0031 \\
\hline $\mathrm{O}$ & -1.208870 & -4.644664 & -1.6 \\
\hline $\mathrm{H}$ & -0.543433 & -5.02 & -2 \\
\hline $\mathrm{H}$ & -1.6774 & & \\
\hline $\mathrm{O}$ & -1.969710 & -3.0 & \\
\hline $\mathrm{H}$ & -2.195157 & & \\
\hline $\mathrm{H}$ & -2.832708 & -2.5 & \\
\hline $\mathrm{O}$ & -4.32 & & \\
\hline $\mathrm{H}$ & -5.124200 & -1.8 & \\
\hline $\mathrm{H}$ & -4.5 & & \\
\hline $\mathrm{O}$ & -4.452887 & -1.0 & -0 . \\
\hline $\mathrm{H}$ & -4.933534 & -0.2 & \\
\hline $\mathrm{H}$ & -4.470614 & -1.7 & \\
\hline $\mathrm{O}$ & -2.860810 & -2.5 & -2 . \\
\hline $\mathrm{H}$ & -3.1416 & -2.5 & -3 . \\
\hline $\mathrm{H}$ & -2.399819 & -3.3 & -2.5 \\
\hline $\mathrm{O}$ & -5.185124 & & -0.9 \\
\hline $\mathrm{H}$ & -6.025238 & 1.9 & -1.2 \\
\hline $\mathrm{H}$ & -4.469321 & & -1. \\
\hline $\mathrm{O}$ & -2.804414 & & -2.0 \\
\hline $\mathrm{H}$ & -2.974884 & 3.7 & -2 \\
\hline $\mathrm{H}$ & -2.255008 & & -2. \\
\hline $\mathrm{O}$ & -0.773188 & 2.0 & -3.8 \\
\hline $\mathrm{H}$ & -0.952504 & & -4.8 \\
\hline $\mathrm{H}$ & 0.087150 & 2.4 & -3. \\
\hline $\mathrm{O}$ & 1.934298 & 2.682688 & -3.18 \\
\hline $\mathrm{H}$ & 2.368188 & $3.4 \mathrm{C}$ & -3.5 \\
\hline $\mathrm{H}$ & 2.465299 & 1.931246 & -3.533154 \\
\hline $\mathrm{O}$ & 2.965484 & 0.072571 & -3.671717 \\
\hline $\mathrm{H}$ & 3.464789 & -0.287446 & -4.421833 \\
\hline
\end{tabular}




\begin{tabular}{|c|c|c|c|}
\hline $\mathrm{H}$ & 276828 & -0.381468 & \\
\hline $\mathrm{O}$ & 2.838572 & -1.075681 & \\
\hline & 3.580962 & -0.631016 & \\
\hline & 3.064704 & -2.050131 & $-0.97^{\prime}$ \\
\hline & 3.500745 & -3.611100 & -0.58 \\
\hline & 4.028033 & -4.260763 & \\
\hline & 3.885955 & -3.535238 & \\
\hline & 4.231555 & -2.384306 & \\
\hline & & -2.751824 & \\
\hline & 4.771467 & -1.609364 & \\
\hline & & -0.0 & \\
\hline & 5.921876 & 0.0 & \\
\hline & & 0.8 & \\
\hline & 4.080913 & 2.4 & \\
\hline & 4.712745 & 3.20 & \\
\hline & & 2.78 & \\
\hline & 2.251776 & 3.2 & 2.9 \\
\hline & 2.41 & 3.7 & \\
\hline & 1.428604 & 3.6 & \\
\hline & -0.200151 & 4.0 & \\
\hline & -0.340313 & 4.9 & \\
\hline & & 3.6 & \\
\hline $\mathrm{O}$ & -2.121198 & 2.5 & \\
\hline & -2.324281 & 2.7 & \\
\hline $\mathrm{H}$ & -2.977094 & 2.2 & \\
\hline & -4.143194 & 1.3 & \\
\hline $\mathrm{H}$ & -4.6781 & 1.5 & \\
\hline & -4.313664 & 0.4 & 2.0 \\
\hline $\mathrm{O}$ & -0.685862 & 9334 & \\
\hline H & -0.520287 & 5031 & \\
\hline & -1.203780 & 4367 & \\
\hline $\mathrm{O}$ & 1.802028 & -1.070292 & \\
\hline & 2.636320 & -1.600281 & \\
\hline $\mathrm{H}$ & 1.332628 & -1.28 & 2.7 \\
\hline $\mathrm{O}$ & -0.060409 & -1.547648 & \\
\hline $\mathrm{H}$ & 0.031496 & -1.565702 & 4.889274 \\
\hline $\mathrm{H}$ & -0.752631 & -2.200990 & 3.67046 \\
\hline $\mathrm{O}$ & -0.684658 & 2.090607 & -0.298580 \\
\hline $\mathrm{H}$ & -0.439421 & 2.868819 & 0.255069 \\
\hline $\mathrm{H}$ & -1.506252 & 2.319875 & -0.788401 \\
\hline $\mathrm{O}$ & 2.145856 & 1.698040 & -0.498322 \\
\hline $\mathrm{H}$ & 2.070266 & 2.226531 & -1.322121 \\
\hline $\mathrm{H}$ & 2.884101 & 2.074650 & 0.047504 \\
\hline $\mathrm{O}$ & 0.428190 & -0.224598 & -2.663320 \\
\hline $\mathrm{H}$ & 1.257018 & -0.266314 & -3.191583 \\
\hline & -0.164800 & 0.396767 & -3.144258 \\
\hline
\end{tabular}




\begin{tabular}{|c|c|c|c|}
\hline $\mathrm{O}$ & -1.850020 & -0.432063 & \\
\hline $\mathrm{H}$ & -1.969538 & -1.089353 & -1.897856 \\
\hline $\mathrm{H}$ & -2.716936 & -0.494872 & \\
\hline \multicolumn{4}{|c|}{$\left[{ }^{1} \mathrm{La}\left(\mathrm{H}_{2} \mathrm{O}\right)_{26}(\mathrm{OH})\right]^{2+}(\mathrm{a})$} \\
\hline \multicolumn{4}{|c|}{$E=-2499.629273$} \\
\hline $\mathrm{O}$ & -2.462034 & 0.694568 & 0.638833 \\
\hline $\mathrm{H}$ & -2.998297 & 1.146793 & \\
\hline $\mathrm{H}$ & -2.639903 & & \\
\hline $\mathrm{O}$ & -2.913779 & 2.434029 & \\
\hline $\mathrm{H}$ & -3.261400 & & \\
\hline $\mathrm{H}$ & -2.2 & 3.1 & \\
\hline $\mathrm{O}$ & -0.8 & & \\
\hline $\mathrm{H}$ & -1.0 & & \\
\hline $\mathrm{H}$ & 0.0 & & \\
\hline $\mathrm{O}$ & 1.6 & & \\
\hline $\mathrm{H}$ & & & \\
\hline $\mathrm{H}$ & 2.4 & & \\
\hline $\mathrm{O}$ & 3.6 & 3.0 & \\
\hline $\mathrm{H}$ & $4.2^{\prime}$ & & \\
\hline $\mathrm{H}$ & 4.1 & 2.2 & \\
\hline $\mathrm{O}$ & 4.3 & & \\
\hline $\mathrm{H}$ & 4.9 & 0.2 & \\
\hline $\mathrm{H}$ & & & \\
\hline $\mathrm{O}$ & 4.5 & -1.6 & \\
\hline $\mathrm{H}$ & 5.20 & -2.3 & \\
\hline $\mathrm{H}$ & 4.4 & -1.5 & \\
\hline $\mathrm{O}$ & 3.8 & -1.6 & \\
\hline $\mathrm{H}$ & 4.4 & -1.3 & \\
\hline $\mathrm{H}$ & $3.5^{\prime}$ & -2.57 & \\
\hline $\mathrm{O}$ & 2.78 & -4.0 & -2 . \\
\hline $\mathrm{H}$ & 3.03 & -4.8 & \\
\hline $\mathrm{H}$ & 1.80 & -3.9 & \\
\hline $\mathrm{O}$ & 0.1 & -3.2 & \\
\hline $\mathrm{H}$ & -0.1 & -3.0 & \\
\hline $\mathrm{H}$ & -0.58 & $-3.5 s$ & -3 \\
\hline $\mathrm{O}$ & -4.26 & & \\
\hline $\mathrm{H}$ & -5.060383 & 2.200076 & -1.1 \\
\hline $\mathrm{H}$ & -4.533239 & 0.78 & \\
\hline $\mathrm{O}$ & -4.319912 & -0.960883 & 3915 \\
\hline $\mathrm{H}$ & -4.733671 & -1.42 & -2.6 \\
\hline $\mathrm{H}$ & -4.568809 & -1.47 & -1.061995 \\
\hline $\mathrm{O}$ & -4.968430 & -2.292686 & \\
\hline $\mathrm{H}$ & -5.817798 & -2.707122 & 0.521309 \\
\hline $\mathrm{H}$ & -4.409859 & -2.347424 & 1.125991 \\
\hline $\mathrm{O}$ & -3.274478 & -2.469340 & 2.545742 \\
\hline
\end{tabular}




\begin{tabular}{|c|c|c|c|}
\hline $\mathrm{H}$ & 3.707712 & -2.532928 & \\
\hline $\mathrm{H}$ & & -3.260693 & \\
\hline $\mathrm{O}$ & -1.296291 & -4.244913 & \\
\hline $\mathrm{H}$ & -1.289068 & -5.212678 & \\
\hline $\mathrm{H}$ & -0.510526 & -3.952221 & \\
\hline 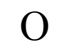 & .763897 & & \\
\hline $\mathrm{H}$ & 1.385047 & -2.5 & \\
\hline 11 & 1.28 & -3.0 & \\
\hline $\mathrm{O}$ & -0.97 & -0.7 & \\
\hline $\mathrm{H}$ & -0.38 & -1.2 & \\
\hline $\mathrm{H}$ & -1.8 & -1.2 & \\
\hline $\mathrm{O}$ & 1.35 & 0.7 & \\
\hline $\mathrm{H}$ & & & \\
\hline $\mathrm{H}$ & 1.33 & 1.6 & \\
\hline $\mathrm{O}$ & 1.85 & -1.9 & \\
\hline $\mathrm{H}$ & 1.23 & -2.4 & \\
\hline $\mathrm{H}$ & 2.7 & -1.9 & \\
\hline $\mathrm{O}$ & -0.3 & & \\
\hline $\mathrm{H}$ & -0.2 & & \\
\hline $\mathrm{H}$ & -0.6 & & \\
\hline $\mathrm{O}$ & -0.3 & & -2.7 \\
\hline $\mathrm{H}$ & -0.3 & & \\
\hline $\mathrm{H}$ & -1.1 & & \\
\hline $\mathrm{O}$ & -2.4 & 2.2 & \\
\hline $\mathrm{H}$ & -2.8 & & \\
\hline $\mathrm{H}$ & -3.2 & 2.2 & -2.8 \\
\hline $\mathrm{O}$ & -1.6 & -0.0 & -2. \\
\hline $\mathrm{H}$ & -2.4 & -0.5 & -1.9 \\
\hline $\mathrm{H}$ & -1.7 & 0.6 & -2.6 \\
\hline $\mathrm{O}$ & 1.1 & -0.7 & -2.1 \\
\hline $\mathrm{H}$ & & & \\
\hline $\mathrm{H}$ & 2.12 & -0.8 & -2.2 \\
\hline $\mathrm{O}$ & 2.2 & & -0.6 \\
\hline $\mathrm{H}$ & 2.55 & & -0.0 \\
\hline $\mathrm{H}$ & 2.25 & & -1.5 \\
\hline $\mathrm{O}$ & 2.25 & 2.63 & $-2.9 c$ \\
\hline $\mathrm{H}$ & 2.6 & 2.49 & -3.8 \\
\hline $\mathrm{H}$ & 1.389 & 3.07 & -3.1 \\
\hline $\mathrm{H}$ & -0.882136 & -2.956522 & 0.3 \\
\hline $\mathrm{O}$ & -0.374847 & -2.363758 & -0.27 \\
\hline LA & -0.028186 & -0.026103 & 0.04 \\
\hline \multicolumn{4}{|c|}{$\left[{ }^{1} \mathrm{La}\left(\mathrm{H}_{2} \mathrm{O}\right)_{26}(\mathrm{OH})\right]^{2+}(\mathrm{b})$} \\
\hline \multicolumn{4}{|c|}{$E=-2499.62446$} \\
\hline $\mathrm{O}$ & & & \\
\hline $\mathrm{H}$ & -2.505096 & 1.798037 & 1.590431 \\
\hline
\end{tabular}




\begin{tabular}{|c|c|c|c|}
\hline H & -1.019722 & 2.101226 & \\
\hline ) & 0.025565 & 3.382971 & \\
\hline & 0.244481 & 3.689416 & 3.334096 \\
\hline & 0.810106 & 3.555243 & 1.877457 \\
\hline & 2.097837 & & \\
\hline & 2.351955 & 4.53 & \\
\hline & 2.941469 & & \\
\hline & 4.593414 & 2.3 & 499 \\
\hline & 5.26 & & \\
\hline & 4.98 & $2.0^{\prime}$ & 573 \\
\hline & 5.39 & & -1.5 \\
\hline & 6.298000 & 1.24 & -1.8 \\
\hline & 5.077 & 0.3 & -1. \\
\hline & 4.099192 & -0.9 & -0.869 \\
\hline & 3.957910 & -1.8 & -1.298 \\
\hline H & 3.18 & -0.5 & -0.8 \\
\hline $\mathrm{O}$ & 1.85 & 0.3 & -0.8 \\
\hline $\mathrm{H}$ & 2.10 & & -1.4 \\
\hline LA & -0.24 & 423 & -0 . \\
\hline $\mathrm{O}$ & 2.977 & 2.43 & -2.4 \\
\hline $\mathrm{H}$ & 2.80 & 2.6 & -3.3 \\
\hline & 3.91 & 2.1 & -2.3 \\
\hline $\mathrm{O}$ & -4.35 & 2.3 & 1.6 \\
\hline $\mathrm{H}$ & -4.68 & 2.7 & 2.4 \\
\hline $\mathrm{H}$ & -4.70 & 1.4 & 1.6 \\
\hline $\mathrm{O}$ & -4.832 & -0.3 & 965 \\
\hline $\mathrm{H}$ & -5.72 & -0.6 & 0.9 \\
\hline $\mathrm{H}$ & -4.386563 & -1.0 & 1.7 \\
\hline $\mathrm{O}$ & -3.37 & -2.3 & 42407 \\
\hline $\mathrm{H}$ & -3.84 & -3.1 & 3424 \\
\hline $\mathrm{H}$ & -2.625786 & -2.21 & 80907 \\
\hline $\mathrm{O}$ & -1.147214 & -1.98 & 53210 \\
\hline $\mathrm{H}$ & -1.1843 & -2.0 & \\
\hline $\mathrm{H}$ & -0.40 & $-2.5^{\prime}$ & 3.666 \\
\hline $\mathrm{O}$ & 1.048731 & -3.30 & \\
\hline $\mathrm{H}$ & 1.323301 & -4.213499 & 3.079419 \\
\hline $\mathrm{H}$ & 1.810491 & -2.71 & 3.101673 \\
\hline $\mathrm{O}$ & 2.765016 & -1.291471 & 3.367983 \\
\hline $\mathrm{H}$ & 3.073971 & -1.134206 & 4.272682 \\
\hline $\mathrm{H}$ & 3.523837 & -0.98 & 2.751256 \\
\hline $\mathrm{O}$ & 4.591889 & -0.440400 & 1.782811 \\
\hline $\mathrm{H}$ & 4.593784 & 0.530312 & 1.663256 \\
\hline $\mathrm{H}$ & 4.507795 & -0.800291 & 0.867770 \\
\hline $\mathrm{O}$ & 2.683914 & -3.430895 & -1.674708 \\
\hline $\mathrm{H}$ & 3.085284 & -4.310576 & -1.619186 \\
\hline $\mathrm{H}$ & 2.050345 & -3.457774 & -2.425961 \\
\hline
\end{tabular}




\begin{tabular}{|c|c|c|c|}
\hline O & 0.560220 & -3.206235 & -3.499494 \\
\hline $\mathrm{H}$ & 0.634566 & -3.484940 & -4.424900 \\
\hline $\mathrm{H}$ & -0.299740 & -3.549574 & -3.164937 \\
\hline $\mathrm{O}$ & -2.024780 & -3.622661 & -2.391515 \\
\hline $\mathrm{H}$ & -2.513085 & -4.457228 & -2.460077 \\
\hline & -2.587211 & -2.931048 & -2.808965 \\
\hline $\mathrm{O}$ & -3.148135 & -1.229881 & -3.3 \\
\hline $\mathrm{H}$ & -3.685172 & -0.994752 & $-4 .($ \\
\hline $\mathrm{H}$ & -3.3452 & -0.579341 & -2.5 \\
\hline $\mathrm{O}$ & -3.0883 & 1565 & -1.1 \\
\hline $\mathrm{H}$ & -3.6082 & & \\
\hline $\mathrm{H}$ & -3.488690 & 1.387018 & -1 . \\
\hline $\mathrm{O}$ & -4.318393 & 3.0 & \\
\hline $\mathrm{H}$ & -5.15 & 3.2 & \\
\hline $\mathrm{H}$ & -4.48 & 2.98 & -0. \\
\hline $\mathrm{O}$ & 0.522 & -0.1 & \\
\hline $\mathrm{H}$ & -0.165 & -0.2 & \\
\hline $\mathrm{H}$ & 1.367 & -0.4 & \\
\hline $\mathrm{O}$ & -0.059767 & 2.4 & -0.8 \\
\hline $\mathrm{H}$ & 0.770 & 2.9 & -0.6 \\
\hline $\mathrm{H}$ & -0.690312 & 3.1 & -1.2 \\
\hline $\mathrm{O}$ & -1.890 & 4.2 & \\
\hline $\mathrm{H}$ & -1.828163 & 5.13 & $-2 .($ \\
\hline $\mathrm{H}$ & -2.81 & 4.0 & -1 . \\
\hline $\mathrm{O}$ & -0.50 & -0.7 & -2 \\
\hline $\mathrm{H}$ & 0.09 & -1.3 & -3. \\
\hline $\mathrm{H}$ & -1.36 & -0.8 & -3 . \\
\hline $\mathrm{O}$ & 0.822 & -2.3598 & 0.1 \\
\hline $\mathrm{H}$ & 0.855366 & -2.787502 & 1.0 \\
\hline $\mathrm{H}$ & 1.555 & -2.7409 & -0. \\
\hline $\mathrm{O}$ & -2.087874 & -1.927153 & \\
\hline $\mathrm{H}$ & -1.9681 & -2.673 & -0.6 \\
\hline $\mathrm{H}$ & -2.527338 & -2.273439 & 0.809486 \\
\hline \multicolumn{4}{|c|}{$\left[{ }^{1} \mathrm{La}\left(\mathrm{H}_{2} \mathrm{O}\right)_{26}(\mathrm{OH})\right]^{2+}(\mathrm{c})$} \\
\hline \multicolumn{4}{|c|}{$E=-2499.62446$} \\
\hline $\mathrm{O}$ & 4.591755 & -0.440524 & \\
\hline $\mathrm{H}$ & 4.592853 & 0.530141 & 1.664342 \\
\hline $\mathrm{H}$ & 4.508623 & -0.800885 & 0.86 \\
\hline $\mathrm{O}$ & 4.592779 & 2.350475 & 0.879975 \\
\hline $\mathrm{H}$ & 5.262192 & 2.904312 & 1.310154 \\
\hline $\mathrm{H}$ & 4.982858 & 2.071329 & 0.014365 \\
\hline $\mathrm{O}$ & 5.393682 & 1.278376 & -1.543108 \\
\hline $\mathrm{H}$ & 6.298508 & 1.244158 & -1.886175 \\
\hline $\mathrm{H}$ & 5.078218 & 0.341161 & -1.430477 \\
\hline $\mathrm{O}$ & 2.978598 & 2.434488 & -2.422478 \\
\hline
\end{tabular}




\begin{tabular}{|c|c|c|c|}
\hline $\mathrm{H}$ & 2.802728 & 2.612400 & -3.356720 \\
\hline H & 3.915204 & 2.157244 & -2.361328 \\
\hline & 2.097347 & 3.674190 & 0.572474 \\
\hline & 2.351922 & 4.538672 & 0.216599 \\
\hline & 2.940776 & 3.166482 & 0.645741 \\
\hline & 0.025378 & 3.385185 & 2.442716 \\
\hline 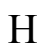 & 0.244201 & 3.691636 & 3.332973 \\
\hline & 0.810143 & 3.556866 & 1.876435 \\
\hline 0 & -1.613345 & 1.398989 & 1.614571 \\
\hline $\mathrm{H}$ & -2.505018 & 1.799115 & 1.589196 \\
\hline $\mathrm{H}$ & -1.019741 & 2.103004 & 1.991872 \\
\hline $\mathrm{O}$ & -4.357041 & 2.324171 & 1.618739 \\
\hline $\mathrm{H}$ & -4.684934 & 2.790424 & 2.402383 \\
\hline $\mathrm{H}$ & -4.708167 & 1.403439 & 1.645528 \\
\hline $\mathrm{O}$ & -4.833998 & -0.331078 & 1.154991 \\
\hline $\mathrm{H}$ & -5.728646 & -0.665401 & 0.989573 \\
\hline $\mathrm{H}$ & -4.389359 & -1.005607 & 1.720560 \\
\hline $\mathrm{O}$ & -3.377113 & -2.352578 & 2.442578 \\
\hline $\mathrm{H}$ & -3.847952 & -3.144180 & 2.743832 \\
\hline $\mathrm{H}$ & -2.627696 & -2.213304 & 3.080955 \\
\hline $\mathrm{O}$ & -1.148990 & -1.985854 & 3.953177 \\
\hline $\mathrm{H}$ & -1.185902 & -2.030812 & 4.920421 \\
\hline $\mathrm{H}$ & -0.406557 & -2.572546 & 3.666378 \\
\hline $\mathrm{O}$ & 1.046486 & -3.306686 & 2.8715 \\
\hline $\mathrm{H}$ & 1.320219 & -4.212464 & 3.081193 \\
\hline $\mathrm{H}$ & 1.808634 & -2.710875 & 3.102856 \\
\hline $\mathrm{O}$ & 0.521831 & -0.158590 & 2.256048 \\
\hline $\mathrm{H}$ & -0.166201 & -0.265595 & 2.935235 \\
\hline $\mathrm{H}$ & 1.367197 & -0.463653 & 2.666596 \\
\hline $\mathrm{O}$ & -4.317702 & 3.033241 & -1.027391 \\
\hline $\mathrm{H}$ & -5.154311 & 3.284632 & -1.446631 \\
\hline $\mathrm{H}$ & -4.479535 & 2.986838 & -0.055759 \\
\hline $\mathrm{O}$ & -2.087665 & -1.926979 & 0.004683 \\
\hline $\mathrm{H}$ & -1.967138 & -2.673634 & -0.615620 \\
\hline $\mathrm{H}$ & -2.528554 & -2.272123 & 0.810037 \\
\hline $\mathrm{O}$ & -2.024199 & -3.623982 & -2.389852 \\
\hline $\mathrm{H}$ & -2.512411 & -4.458597 & -2.458424 \\
\hline $\mathrm{H}$ & -2.586675 & -2.932436 & -2.807328 \\
\hline $\mathrm{O}$ & -3.147490 & -1.231262 & -3.314125 \\
\hline $\mathrm{H}$ & -3.684371 & -0.996501 & -4.085306 \\
\hline $\mathrm{H}$ & -3.344584 & -0.580298 & -2.596821 \\
\hline $\mathrm{O}$ & -3.088263 & 0.491124 & -1.159408 \\
\hline $\mathrm{H}$ & -3.608348 & 0.055252 & -0.452852 \\
\hline $\mathrm{H}$ & -3.488625 & 1.386527 & -1.254346 \\
\hline $\mathrm{O}$ & -0.506191 & -0.712569 & -2.637541 \\
\hline $\mathrm{H}$ & 0.099954 & -1.366523 & -3.040037 \\
\hline
\end{tabular}




$\begin{array}{lccc}\mathrm{H} & -1.363653 & -0.817659 & -3.099742 \\ \mathrm{O} & 0.560786 & -3.207760 & -3.498276 \\ \mathrm{H} & 0.634353 & -3.487188 & -4.423522 \\ \mathrm{H} & -0.298987 & -3.550677 & -3.162814 \\ \mathrm{O} & 2.684564 & -3.431347 & -1.673612 \\ \mathrm{H} & 3.085853 & -4.311031 & -1.617582 \\ \mathrm{H} & 2.051100 & -3.458571 & -2.424941 \\ \mathrm{O} & 0.821873 & -2.360275 & 0.128221 \\ \mathrm{H} & 0.854509 & -2.787599 & 1.007873 \\ \mathrm{H} & 1.555997 & -2.740981 & -0.403576 \\ \mathrm{O} & -1.889990 & 4.201176 & -1.826605 \\ \mathrm{H} & -1.826886 & 5.138567 & -2.053844 \\ \mathrm{H} & -2.811312 & 4.017440 & -1.555307 \\ \mathrm{O} & -0.059138 & 2.457184 & -0.892191 \\ \mathrm{H} & 0.770492 & 2.904622 & -0.620510 \\ \mathrm{H} & -0.689739 & 3.148313 & -1.216444 \\ \mathrm{O} & 1.851497 & 0.304552 & -0.855023 \\ \mathrm{H} & 2.108129 & 1.040539 & -1.451293 \\ \mathrm{LA} & -0.249146 & 0.038379 & -0.195749 \\ \mathrm{H} & 3.523125 & -0.985500 & 2.752351 \\ \mathrm{O} & 2.764007 & -1.291447 & 3.368750 \\ \mathrm{H} & 3.072578 & -1.133924 & 4.273542 \\ \mathrm{H} & 3.182493 & -0.533415 & -0.857979 \\ \mathrm{O} & 4.099923 & -0.978227 & -0.868172 \\ \mathrm{H} & 3.958710 & -1.840707 & -1.297719\end{array}$

\begin{tabular}{crrr}
\multicolumn{5}{c}{$\left[{ }^{1} \mathrm{La}\left(\mathrm{H}_{2} \mathrm{O}\right){ }_{26}(\mathrm{OH})\right]^{2+}(\mathrm{d})$} \\
$\mathrm{E}=-2499.622711$ \\
$\mathrm{O}$ & -2.056172 & 0.931868 & 1.588136 \\
$\mathrm{H}$ & -2.901153 & 1.318631 & 1.273537 \\
$\mathrm{H}$ & -1.662513 & 1.621433 & 2.175528 \\
$\mathrm{O}$ & -0.801772 & 2.816877 & 3.186908 \\
$\mathrm{H}$ & -1.174097 & 3.494979 & 3.767572 \\
$\mathrm{H}$ & -0.057348 & 3.229004 & 2.705725 \\
$\mathrm{O}$ & 1.125118 & 4.013707 & 1.322601 \\
$\mathrm{H}$ & 1.090983 & 4.982079 & 1.305610 \\
$\mathrm{H}$ & 2.071544 & 3.776184 & 1.180807 \\
$\mathrm{O}$ & 3.809505 & 3.296768 & 0.714701 \\
$\mathrm{H}$ & 4.477327 & 3.982105 & 0.873095 \\
$\mathrm{H}$ & 3.883066 & 3.045587 & -0.237481 \\
$\mathrm{O}$ & 3.907656 & 2.090462 & -1.758062 \\
$\mathrm{H}$ & 4.088353 & 2.512516 & -2.611925 \\
$\mathrm{H}$ & 4.452923 & 1.267088 & -1.721245 \\
$\mathrm{O}$ & 5.175807 & -0.313634 & -1.270433 \\
$\mathrm{H}$ & 6.078701 & -0.503155 & -1.568837 \\
$\mathrm{H}$ & 4.644552 & -1.120360 & -1.461451
\end{tabular}




\begin{tabular}{|c|c|c|c|}
\hline $\mathrm{O}$ & -4.623023 & 1.822484 & 0.715193 \\
\hline $\mathrm{H}$ & -5.145503 & 2.395301 & 1.296562 \\
\hline H & -4.931264 & 0.897358 & 0.874104 \\
\hline $\int$ & -4.858682 & -0.852655 & 1.073725 \\
\hline $\mathrm{H}$ & -5.638609 & -1.417664 & 0.977402 \\
\hline & -4.289176 & -1.265780 & 1.760967 \\
\hline $\mathrm{O}$ & -3.040026 & -2.233890 & 2.765393 \\
\hline $\mathrm{H}$ & -3.387782 & -2.971494 & 3.288939 \\
\hline $\mathrm{H}$ & -2.325252 & -1.827338 & 3.311708 \\
\hline $\mathrm{O}$ & -0.691581 & -1.356490 & 4.019974 \\
\hline $\mathrm{H}$ & -0.635518 & -1.263230 & 4.982776 \\
\hline $\mathrm{H}$ & 0.058977 & -1.935655 & 3.747857 \\
\hline $\mathrm{O}$ & 1.672543 & -2.584643 & 3.041636 \\
\hline $\mathrm{H}$ & 2.032947 & -3.425851 & 3.358943 \\
\hline $\mathrm{H}$ & 2.345931 & -1.885914 & 3.232796 \\
\hline $\mathrm{O}$ & 3.087122 & -0.284539 & 3.252079 \\
\hline $\mathrm{H}$ & 3.370655 & 0.080335 & 4.103673 \\
\hline $\mathrm{H}$ & 3.783055 & -0.004853 & 2.584627 \\
\hline $\mathrm{O}$ & 4.778295 & 0.666302 & 1.482714 \\
\hline $\mathrm{H}$ & 5.008672 & 0.252747 & 0.629507 \\
\hline $\mathrm{H}$ & 4.520356 & 1.589640 & 1.289545 \\
\hline $\mathrm{O}$ & 3.637937 & -2.695521 & -1.398192 \\
\hline $\mathrm{H}$ & 4.168112 & -3.447540 & -1.092917 \\
\hline $\mathrm{H}$ & 2.953975 & -3.076215 & -2.001887 \\
\hline $\mathrm{O}$ & 1.438646 & -3.666886 & -2.775928 \\
\hline $\mathrm{H}$ & 1.503174 & -4.178585 & -3.596562 \\
\hline $\mathrm{H}$ & 0.697742 & -4.060136 & -2.255065 \\
\hline $\mathrm{O}$ & -0.892872 & -4.354199 & -1.315028 \\
\hline $\mathrm{H}$ & -1.168697 & -5.273230 & -1.178061 \\
\hline $\mathrm{H}$ & -1.645440 & -3.895265 & -1.777745 \\
\hline $\mathrm{O}$ & 0.596793 & 0.399267 & 2.105356 \\
\hline $\mathrm{H}$ & 0.021173 & 0.177518 & 2.863577 \\
\hline $\mathrm{H}$ & 1.511040 & 0.253291 & 2.435270 \\
\hline $\mathrm{O}$ & 1.642275 & -1.601153 & 0.272028 \\
\hline $\mathrm{H}$ & 1.574479 & -2.118496 & 1.100662 \\
\hline $\mathrm{H}$ & 2.388020 & -1.965652 & -0.247491 \\
\hline $\mathrm{O}$ & -0.977254 & -2.447118 & 0.861973 \\
\hline $\mathrm{H}$ & -1.802030 & -2.548416 & 1.381663 \\
\hline $\mathrm{H}$ & -0.933218 & -3.196514 & 0.231221 \\
\hline $\mathrm{O}$ & -0.457435 & 2.486396 & -0.449957 \\
\hline $\mathrm{H}$ & 0.051579 & 3.106065 & 0.115072 \\
\hline $\mathrm{H}$ & -0.888023 & 2.997506 & -1.175824 \\
\hline $\mathrm{O}$ & -1.783557 & 3.532573 & -2.611745 \\
\hline $\mathrm{H}$ & -1.782155 & 4.466437 & -2.869664 \\
\hline $\mathrm{H}$ & -2.733501 & 3.307567 & -2.402016 \\
\hline $\mathrm{O}$ & -4.281304 & 2.988746 & -1.850125 \\
\hline
\end{tabular}




$\begin{array}{cccc}\mathrm{H} & -5.063907 & 2.874474 & -2.406077 \\ \mathrm{H} & -4.452999 & 2.524996 & -1.002617 \\ \mathrm{O} & 0.097252 & 1.887010 & -3.914026 \\ \mathrm{H} & -0.168310 & 1.509709 & -4.763935 \\ \mathrm{H} & -0.640723 & 2.459095 & -3.611548 \\ \mathrm{O} & 1.431326 & 0.641669 & -1.895856 \\ \mathrm{H} & 2.200538 & 1.228269 & -1.756775 \\ \mathrm{H} & 0.978288 & 0.999650 & -2.702795 \\ \mathrm{O} & -0.282932 & -1.367443 & -2.499397 \\ \mathrm{H} & 0.475269 & -1.914897 & -2.773456 \\ \mathrm{H} & -1.095725 & -1.841477 & -2.785535 \\ \mathrm{O} & -2.560913 & -0.560940 & -0.992977 \\ \mathrm{H} & -3.357552 & -0.542167 & -0.437534 \\ \mathrm{LA} & -0.454334 & -0.086708 & -0.232605 \\ \mathrm{H} & -2.769943 & -1.808519 & -1.864295 \\ \mathrm{O} & -2.653571 & -2.654552 & -2.448745 \\ \mathrm{H} & -3.397437 & -2.722519 & -3.063067 \\ & & & \\ {\left[{ }^{1} \mathrm{La}\left(\mathrm{H}_{2} \mathrm{O}\right)_{26}(\mathrm{OH})\right]^{2+}(\mathrm{e})} & \\ \mathrm{E}= & -2499.617391 & & \\ \mathrm{O} & 1.953689 & -1.176945 & -0.786260 \\ \mathrm{H} & 2.709267 & -0.553662 & -0.846349 \\ \mathrm{H} & 2.023614 & -1.630420 & -1.653498 \\ \mathrm{O} & 2.964687 & -1.642310 & -3.401359 \\ \mathrm{H} & 3.253232 & -2.374299 & -3.964468 \\ \mathrm{H} & 2.238323 & -1.162511 & -3.895983 \\ \mathrm{O} & 0.866413 & -0.329897 & -4.306974 \\ \mathrm{H} & -0.875523 & 1.617887 & 3.972903 \\ \mathrm{H} & 0.691255 & 0.149241 & -5.127356 \\ \mathrm{H} & 0.591880 & 0.251458 & -3.541131 \\ \mathrm{O} & -3.845878 & 0.365201 & -3.392955 \\ \mathrm{H} & -4.239270 & 0.176742 & -4.255091 \\ \mathrm{H} & -4.255048 & 1.177197 & -3.032737 \\ \mathrm{O} & -4.590259 & 2.278186 & -1.504965 \\ \mathrm{H} & -5.056013 & 3.126598 & -1.545102 \\ \mathrm{H} & -5.013260 & 1.741216 & -0.799119 \\ \mathrm{O} & -4.997029 & 0.347157 & 0.428958 \\ \mathrm{H} & -5.838906 & -0.111223 & 0.572051 \\ \mathrm{H} & -4.644196 & 0.563736 & 1.323493 \\ \mathrm{O} & -3.764174 & 0.627980 & 2.947666 \\ -4.289842 & 0.494441 & 3.750518 \\ \mathrm{H} & -1.589993 & 1.282901 & 3.183480 \\ \mathrm{H} & -1.672877 & 2.171532 & 3.576431 \\ \mathrm{H} & 0.257720 & 5.173643\end{array}$




\begin{tabular}{|c|c|c|c|}
\hline & 1.385560 & 1.135531 & \\
\hline & 2.417585 & 2.156027 & 3.034770 \\
\hline & .212156 & 1.823782 & 2.508756 \\
\hline & 2.678446 & 3.017697 & 3.393807 \\
\hline & 4.335769 & 0.010994 & -1.671912 \\
\hline & 4.102437 & -0.540522 & -2.454606 \\
\hline & 4.877603 & -0.535459 & -1.0 \\
\hline & 5.146229 & -1.257663 & 0.7 \\
\hline & 6.001075 & -1.637570 & \\
\hline & 4.457028 & -1.893284 & 1.0 \\
\hline & 3.018716 & -2.84 & \\
\hline & 3.262967 & -3.273149 & 2.6 \\
\hline & 2.412417 & -3.470044 & \\
\hline ) & 0.918233 & -4.405959 & 0.7 \\
\hline & 1.066157 & -5.3 & 0.4 \\
\hline & 0.05 & -4.2 & \\
\hline & -1.763902 & -4.0 & 1.6 \\
\hline & -2.204385 & -4.5 & 2.2 \\
\hline $\mathrm{H}$ & -2.305734 & -4.032195 & 0.7 \\
\hline $\mathrm{O}$ & -2.932227 & -3.626976 & -0.9 \\
\hline $\mathrm{H}$ & -3.154297 & -2.680834 & -1.0 \\
\hline & -3.48 & -4.1 & -1. \\
\hline $\mathrm{O}$ & -0.366657 & -2.5 & -1.0 \\
\hline & -1.170251 & 2601 & -1.2 \\
\hline $\mathrm{H}$ & 0.2575 & -3.1 & -0.6 \\
\hline $\mathrm{O}$ & -2.903133 & -0.869 & -1.2 \\
\hline $\mathrm{H}$ & -3.573225 & -0.5 & -0.5 \\
\hline $\mathrm{H}$ & -3.206809 & -0.5 & -2.1 \\
\hline $\mathrm{O}$ & -2.092852 & -1.099831 & 1.5 \\
\hline $\mathrm{H}$ & -1.952244 & -2.023663 & 1.8 \\
\hline $\mathrm{H}$ & -2.707801 & -0.6 & 2.1 \\
\hline $\mathrm{O}$ & 0.843799 & -0.9927 & 1.8 \\
\hline $\mathrm{H}$ & 0.705158 & -0.608042 & 2.7 \\
\hline $\mathrm{H}$ & 1.667730 & -1.5218 & 1.8 \\
\hline $\mathrm{O}$ & 0.279198 & 1.836687 & 1.27 \\
\hline $\mathrm{H}$ & 1.088111 & 2.014044 & 1.807104 \\
\hline $\mathrm{H}$ & -0.434944 & 2.340036 & 1.705208 \\
\hline $\mathrm{O}$ & 4.379493 & 1.421010 & 1.495826 \\
\hline $\mathrm{H}$ & 4.731546 & 0.525860 & 1.31 \\
\hline $\mathrm{H}$ & 4.390866 & 1.914292 & 0.64 \\
\hline $\mathrm{O}$ & 4.194862 & 2.642093 & -1.106408 \\
\hline $\mathrm{H}$ & 4.969030 & 3.163345 & -1.368942 \\
\hline $\mathrm{H}$ & 4.312702 & 1.741698 & -1.503094 \\
\hline $\mathrm{C}$ & -2.052412 & 1.969414 & -0.187904 \\
\hline $\mathrm{H}$ & -2.870270 & 2.097488 & -0.711795 \\
\hline & -1.560216 & 2.828257 & -0.275159 \\
\hline
\end{tabular}




$\begin{array}{llll}\mathrm{O} & -0.596589 & 4.269721 & -0.330544 \\ \mathrm{H} & -0.913002 & 5.158455 & -0.543900 \\ \mathrm{H} & 0.245160 & 4.142899 & -0.822648 \\ \mathrm{O} & 1.669486 & 3.671424 & -1.910553 \\ \mathrm{H} & 1.823300 & 4.227272 & -2.688534 \\ \mathrm{H} & 2.559233 & 3.370391 & -1.612141 \\ \mathrm{H} & 0.639583 & 1.924306 & -2.181059 \\ \mathrm{O} & 0.283043 & 1.024604 & -2.073902 \\ \mathrm{LA} & -0.418678 & -0.087839 & -0.304099\end{array}$

$\left[{ }^{1} \mathrm{La}\left(\mathrm{H}_{2} \mathrm{O}\right)_{26}(\mathrm{OH})\right]^{2+}$ (f)

$\mathrm{E}=-2499.617173$

$\begin{array}{lrrr}\text { LA } & -0.310000 & 0.170850 & -0.367985 \\ \mathrm{O} & 0.831753 & 2.459919 & 0.109974 \\ \mathrm{O} & 0.068910 & -0.104274 & 2.139527 \\ \mathrm{H} & 1.159673 & 2.746519 & 0.992153 \\ \mathrm{H} & 1.282296 & 3.017115 & -0.558337 \\ \mathrm{H} & 0.778177 & -0.694409 & 2.488420 \\ \mathrm{H} & -0.665368 & -0.173802 & 2.785883 \\ \mathrm{O} & 2.123253 & 3.716110 & -2.129179 \\ \mathrm{H} & 1.784590 & 2.918238 & -2.589640 \\ \mathrm{H} & 1.744808 & 4.489776 & -2.572936 \\ \mathrm{O} & 1.885423 & -1.803595 & 3.412355 \\ \mathrm{H} & 2.212929 & -1.473953 & 4.263081 \\ \mathrm{H} & 2.703591 & -1.861253 & 2.835096 \\ \mathrm{O} & 4.117562 & -1.545094 & 2.017861 \\ \mathrm{H} & 4.331094 & -1.857323 & 1.117231 \\ \mathrm{H} & 4.184513 & -0.570894 & 1.968795 \\ \mathrm{O} & 4.054849 & 1.316224 & 1.568102 \\ \mathrm{H} & 4.935227 & 1.717862 & 1.634799 \\ \mathrm{H} & 3.906571 & 1.157744 & 0.598701 \\ \mathrm{O} & 3.776334 & 0.669328 & -1.111619 \\ \mathrm{H} & 4.134260 & -0.247667 & -1.154184 \\ \mathrm{H} & 4.395381 & 1.296475 & -1.558489 \\ \mathrm{O} & 4.889069 & 2.936297 & -2.009215 \\ \mathrm{H} & 5.572501 & 3.312134 & -2.580716 \\ \mathrm{H} & 4.070674 & 3.453769 & -2.143560 \\ \mathrm{O} & 4.596332 & -2.007599 & -0.830773 \\ \mathrm{H} & 5.449076 & -2.358888 & -1.127879 \\ \mathrm{H} & 3.917707 & -2.686377 & -1.059845 \\ \mathrm{O} & 2.567288 & -3.864998 & -1.085431 \\ \mathrm{H} & 2.704378 & -4.759801 & -0.742670 \\ \mathrm{H} & 1.823850 & -3.914533 & -1.724547 \\ \mathrm{O} & 0.370400 & -3.755726 & -2.881095 \\ \mathrm{H} & 0.491047 & -4.205662 & -3.731396 \\ \mathrm{H} & -0.549813 & -3.948336 & -2.590865\end{array}$




\begin{tabular}{|c|c|c|c|}
\hline $\mathrm{O}$ & -2.337174 & -3.648785 & -2.092096 \\
\hline H & -2.941429 & -4.406189 & -2.062508 \\
\hline & -2.741683 & -2.980307 & -2.693088 \\
\hline O & -3.010196 & -1.370374 & -3.561797 \\
\hline & -3.550515 & -1.230823 & -4.353423 \\
\hline $\mathrm{H}$ & -3.113893 & -0.578378 & -2.978466 \\
\hline 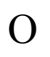 & -2.771109 & 0.689788 & -1.733060 \\
\hline $\mathrm{H}$ & -3.484559 & 0.520211 & -1.078119 \\
\hline $\mathrm{H}$ & -2.927381 & 1.625539 & -2.038371 \\
\hline $\mathrm{O}$ & -3.197333 & 3.295100 & -2.184448 \\
\hline $\mathrm{H}$ & -3.608346 & 3.803525 & -2.897420 \\
\hline $\mathrm{H}$ & -3.646746 & 3.538311 & -1.348098 \\
\hline $\mathrm{O}$ & -4.212173 & 3.156376 & 0.414023 \\
\hline $\mathrm{H}$ & -4.666002 & 3.800705 & 0.977211 \\
\hline $\mathrm{H}$ & -4.721770 & 2.316164 & 0.453836 \\
\hline $\mathrm{O}$ & -4.919849 & 0.488336 & 0.217281 \\
\hline $\mathrm{H}$ & -5.801331 & 0.178578 & -0.040299 \\
\hline $\mathrm{H}$ & -4.629016 & -0.093495 & 0.961284 \\
\hline $\mathrm{O}$ & -3.940094 & -1.373681 & 2.058256 \\
\hline $\mathrm{H}$ & -4.538628 & -2.057212 & 2.395645 \\
\hline $\mathrm{H}$ & -3.312763 & -1.167946 & 2.804124 \\
\hline $\mathrm{O}$ & -2.004434 & -0.963504 & 96494 \\
\hline $\mathrm{H}$ & -2.121455 & -0.490164 & 4.733582 \\
\hline $\mathrm{H}$ & -1.509551 & -1.807236 & 4.096394 \\
\hline $\mathrm{O}$ & -0.464852 & -3.126385 & 4.080377 \\
\hline $\mathrm{H}$ & -0.414582 & -3.887914 & \\
\hline $\mathrm{H}$ & 0.452168 & -2.888437 & 3.818201 \\
\hline $\mathrm{O}$ & 1.992516 & 2.888052 & 2.586131 \\
\hline $\mathrm{H}$ & 2.379145 & 3.729923 & 2.870008 \\
\hline $\mathrm{H}$ & 2.753954 & 2.301538 & 2.350266 \\
\hline $\mathrm{O}$ & -1.953304 & 1.578966 & 1.202399 \\
\hline $\mathrm{H}$ & -2.622427 & 2.244461 & 0.944815 \\
\hline $\mathrm{H}$ & -1.547197 & 1.918267 & 2.037629 \\
\hline $\mathrm{O}$ & -0.706739 & 2.413466 & 3.531615 \\
\hline $\mathrm{H}$ & -0.935733 & 3.178989 & 4.077421 \\
\hline $\mathrm{H}$ & 0.255651 & 2.472902 & 3.359970 \\
\hline $\mathrm{O}$ & -2.102606 & -1.682993 & -0.020146 \\
\hline $\mathrm{H}$ & -2.165329 & -2.497384 & -0.558686 \\
\hline $\mathrm{H}$ & -2.750717 & -1.746701 & 0.715268 \\
\hline $\mathrm{O}$ & -0.334721 & -0.986797 & -2.871509 \\
\hline $\mathrm{H}$ & -1.151840 & -1.098196 & -3.398133 \\
\hline $\mathrm{H}$ & 0.093935 & -1.869887 & -2.856773 \\
\hline $\mathrm{O}$ & 1.379318 & 1.081866 & -2.321929 \\
\hline $\mathrm{H}$ & 1.142161 & 0.413003 & -2.989557 \\
\hline $\mathrm{H}$ & 2.261806 & 0.816721 & -1.936557 \\
\hline $\mathrm{H}$ & 1.668601 & -2.166933 & -0.203577 \\
\hline
\end{tabular}




\begin{tabular}{|c|c|c|c|}
\hline $\mathrm{O}$ & 1.201359 & .329378 & \\
\hline \multicolumn{4}{|c|}{$\left[{ }^{1} \mathrm{La}\left(\mathrm{H}_{2} \mathrm{O}\right)_{26}(\mathrm{OH})\right]^{2+}(\mathrm{g})$} \\
\hline \multicolumn{4}{|c|}{$E=-2499.608099$} \\
\hline LA & 0.593180 & 0.143989 & -0.017286 \\
\hline $\mathrm{O}$ & -0.384774 & -0.588288 & \\
\hline $\mathrm{O}$ & -0.215884 & -2.234844 & \\
\hline $\mathrm{H}$ & -0.833636 & -1.454757 & -2.585485 \\
\hline $\mathrm{H}$ & -0.639110 & -0.050 & \\
\hline H & -0.99 & -2.46 & \\
\hline $\mathrm{H}$ & 0.5 & -2.69 & \\
\hline $\mathrm{O}$ & -1.24 & 1.128 & -4 \\
\hline $\mathrm{H}$ & -0.51 & 1.702 & \\
\hline $\mathrm{H}$ & -1.5 & 0.666 & \\
\hline $\mathrm{O}$ & -2.2 & $-2.91^{\prime}$ & \\
\hline $\mathrm{H}$ & $-2.4^{\prime}$ & -3.8 & \\
\hline $\mathrm{H}$ & & & \\
\hline $\mathrm{O}$ & -4.4 & -1.8 & \\
\hline $\mathrm{H}$ & -4.6 & -0.9 & \\
\hline $\mathrm{H}$ & -4.4 & -2.1 & \\
\hline $\mathrm{O}$ & -4.31 & -2.4 & \\
\hline $\mathrm{H}$ & -5.0 & -2.8 & \\
\hline $\mathrm{H}$ & -4.4 & -1.4 & \\
\hline $\mathrm{O}$ & -4.4 & 0.2 & \\
\hline $\mathrm{H}$ & -4.85 & 0.6 & \\
\hline $\mathrm{H}$ & -4.25 & & \\
\hline $\mathrm{O}$ & -3.1 & 2.24 & \\
\hline $\mathrm{H}$ & -3.5 & 3.08 & \\
\hline $\mathrm{H}$ & -2.5 & 1.9 & \\
\hline $\mathrm{O}$ & -5.04 & 0.99 & \\
\hline $\mathrm{H}$ & -5.8 & 1.2 & \\
\hline $\mathrm{H}$ & -4.3 & 1.56 & \\
\hline $\mathrm{O}$ & -2.9 & 2.39 & \\
\hline $\mathrm{H}$ & -3.0 & 2.68 & \\
\hline $\mathrm{H}$ & -2.3 & 3.1 & \\
\hline $\mathrm{O}$ & -1.2 & 4.27 & \\
\hline $\mathrm{H}$ & -1.6 & 5.11 & \\
\hline $\mathrm{H}$ & -0.38 & 4.49 & \\
\hline $\mathrm{O}$ & 1.23 & 4.67 & \\
\hline $\mathrm{H}$ & 1.627260 & 5.176407 & \\
\hline $\mathrm{H}$ & 1.94 & 4.41 & \\
\hline $\mathrm{O}$ & 2.811886 & 3.881 & -0.0 \\
\hline $\mathrm{H}$ & 3.360621 & 4.515628 & -0.5 \\
\hline $\mathrm{H}$ & 3.115943 & 2.976129 & -0.2 \\
\hline $\mathrm{O}$ & 3.003476 & 1.243111 & -0.856512 \\
\hline $\mathrm{H}$ & 3.627766 & 0.774631 & -0.255613 \\
\hline
\end{tabular}




\begin{tabular}{|c|c|c|c|}
\hline $\mathrm{H}$ & & & \\
\hline $\mathrm{O}$ & & & \\
\hline $\mathrm{H}$ & & .037688 & \\
\hline $\mathrm{H}$ & & & \\
\hline $\mathrm{O}$ & 4.737665 & & \\
\hline $\mathrm{H}$ & & & \\
\hline $\mathrm{H}$ & & -1.290043 & \\
\hline $\mathrm{O}$ & & & \\
\hline $\mathrm{H}$ & & & \\
\hline $\mathrm{H}$ & & -0.7 & \\
\hline $\mathrm{O}$ & & -1.5 & \\
\hline $\mathrm{H}$ & 33 & -1.9 & \\
\hline $\mathrm{H}$ & & -2.0 & \\
\hline $\mathrm{O}$ & 79 & -2.9 & \\
\hline $\mathrm{H}$ & & -3.8 & \\
\hline $\mathrm{H}$ & & -2.4 & \\
\hline $\mathrm{O}$ & & -1. & \\
\hline $\mathrm{H}$ & & -1.5 & 5.0 \\
\hline $\mathrm{H}$ & & & \\
\hline $\mathrm{O}$ & & -3. & -2.6 \\
\hline $\mathrm{H}$ & -2.0 & -3. & -3.4 \\
\hline $\mathrm{H}$ & & -2.9 & \\
\hline $\mathrm{O}$ & 2.0 & -1.8 & -0. \\
\hline $\mathrm{H}$ & 5 & -1.8 & \\
\hline $\mathrm{H}$ & & -2.6 & \\
\hline $\mathrm{O}$ & & & \\
\hline $\mathrm{H}$ & & -4.8 & -2.2 \\
\hline $\mathrm{H}$ & -0.2 & -3.8 & -1. \\
\hline $\mathrm{O}$ & & & \\
\hline $\mathrm{H}$ & -0.7 & -0 . & 16 \\
\hline $\mathrm{H}$ & -1.7 & & \\
\hline $\mathrm{O}$ & & & -0.5 \\
\hline $\mathrm{H}$ & 51 & 3.2 & -0.5 \\
\hline $\mathrm{H}$ & -0.3 & & \\
\hline $\mathrm{O}$ & & & \\
\hline $\mathrm{H}$ & -1.9 & & \\
\hline $\mathrm{H}$ & -2.66 & & \\
\hline $\mathrm{H}$ & & & \\
\hline $\mathrm{O}$ & 1.913200 & 0.266977 & \\
\hline \multicolumn{4}{|c|}{$\left[{ }^{1} \mathrm{La}\left(\mathrm{H}_{2} \mathrm{O}\right)_{26}(\mathrm{OH})\right]^{2+}(\mathrm{h})$} \\
\hline \multicolumn{4}{|c|}{$E=-2499.607717$} \\
\hline & 0.698955 & 0.052556 & \\
\hline $\mathrm{O}$ & & 0.154401 & -2.480384 \\
\hline $\mathrm{O}$ & -1.045530 & -1.791949 & -0.411501 \\
\hline $\mathrm{H}$ & -1.318186 & -0.512368 & -2.553238 \\
\hline
\end{tabular}




\begin{tabular}{|c|c|c|c|}
\hline $\mathrm{H}$ & -0.813594 & 0.866348 & -3.110038 \\
\hline & -1.707420 & -2.208794 & 0.198937 \\
\hline & -0.425909 & -2.486952 & -0.696238 \\
\hline & -1.390713 & 2.502987 & -4.143195 \\
\hline & -0.627283 & 2.943962 & -4.549541 \\
\hline & -1.999557 & 2.321355 & -4.877148 \\
\hline & -2.785991 & -3.070174 & 1.202603 \\
\hline & -3.033473 & -3.679592 & 0.470855 \\
\hline $\mathrm{H}$ & -3.557365 & -2.459339 & 1.347903 \\
\hline$U$ & -4.756390 & -1.243616 & 1.345104 \\
\hline $\mathrm{H}$ & -4.663504 & -0.372085 & 1.770842 \\
\hline H & -5.041527 & -1.077408 & 0.425898 \\
\hline 0 & -5.173015 & -0.540835 & -1.473873 \\
\hline $\mathrm{H}$ & -6.082664 & -0.562931 & -1.806985 \\
\hline $\mathrm{H}$ & -4.971863 & 0.395277 & -1.228255 \\
\hline $\mathrm{O}$ & -4.235295 & 1.767579 & -0.448322 \\
\hline $\mathrm{H}$ & -4.449442 & 1.956164 & 0.491104 \\
\hline $\mathrm{H}$ & -3.954181 & 2.596188 & -0.882379 \\
\hline $\mathrm{O}$ & -2.487211 & 3.649285 & -1.732231 \\
\hline $\mathrm{H}$ & -2.534633 & 4.616491 & -1.751536 \\
\hline $\mathrm{H}$ & -2.171461 & 3.371445 & -2.617017 \\
\hline $\mathrm{O}$ & -4.341613 & 1.573681 & 2.319788 \\
\hline $\mathrm{H}$ & -5.000965 & 1.902222 & 2.949456 \\
\hline $\mathrm{H}$ & -3.460700 & 1.741771 & 2.722299 \\
\hline $\mathrm{O}$ & -1.700649 & 1.851008 & 3.433939 \\
\hline $\mathrm{H}$ & -1.666797 & 1.864474 & 4.403009 \\
\hline $\mathrm{H}$ & -1.056971 & 2.533941 & 3.135264 \\
\hline $\mathrm{O}$ & 0.346416 & 3.655530 & 2.569049 \\
\hline $\mathrm{H}$ & 0.219222 & 4.593462 & 2.779342 \\
\hline $\mathrm{H}$ & 1.282285 & 3.445010 & 2.801675 \\
\hline $\mathrm{O}$ & 3.030623 & 2.864281 & 2.697850 \\
\hline $\mathrm{H}$ & 3.666006 & 3.098993 & 3.391259 \\
\hline $\mathrm{H}$ & 3.453336 & 3.085846 & 1.833444 \\
\hline $\mathrm{O}$ & 3.748959 & 3.205182 & 0.030756 \\
\hline $\mathrm{H}$ & 4.365780 & 3.825347 & -0.384877 \\
\hline $\mathrm{H}$ & 3.774932 & 2.356796 & -0.487106 \\
\hline $\mathrm{O}$ & 3.227619 & 0.861707 & -1.222723 \\
\hline $\mathrm{H}$ & 3.778233 & 0.146192 & -0.831347 \\
\hline $\mathrm{H}$ & 3.320044 & 0.716542 & -2.205560 \\
\hline $\mathrm{O}$ & 3.407404 & 0.087097 & -3.782954 \\
\hline $\mathrm{H}$ & 4.016162 & 0.357310 & -4.484372 \\
\hline $\mathrm{H}$ & 3.551833 & -0.875737 & -3.634968 \\
\hline $\mathrm{O}$ & 3.836836 & -2.453460 & -2.747707 \\
\hline $\mathrm{H}$ & 4.150023 & -3.284449 & -3.132608 \\
\hline $\mathrm{H}$ & 4.391821 & -2.261494 & -1.960490 \\
\hline $\mathrm{O}$ & 4.903461 & -1.385324 & -0.400387 \\
\hline
\end{tabular}




$\begin{array}{crcc}\mathrm{H} & 5.852777 & -1.280169 & -0.237751 \\ \mathrm{H} & 4.524990 & -1.783395 & 0.419930 \\ \mathrm{O} & 3.773003 & -2.173402 & 2.047371 \\ \mathrm{H} & 4.358928 & -2.375522 & 2.792465 \\ \mathrm{H} & 3.032971 & -2.842631 & 2.108393 \\ \mathrm{O} & 1.800569 & -3.926379 & 2.461459 \\ \mathrm{H} & 1.788726 & -4.833611 & 2.126768 \\ \mathrm{H} & 0.867015 & -3.660101 & 2.607062 \\ \mathrm{O} & -0.812833 & -2.965416 & 3.009919 \\ \mathrm{H} & -1.069569 & -3.238891 & 3.903616 \\ \mathrm{H} & -1.584042 & -3.175439 & 2.416968 \\ \mathrm{O} & -3.007079 & -1.613286 & -2.905160 \\ \mathrm{H} & -3.257487 & -1.753637 & -3.830551 \\ \mathrm{H} & -3.804043 & -1.233718 & -2.463134 \\ \mathrm{O} & -0.399416 & -0.338065 & 2.136246 \\ \mathrm{H} & -0.543896 & -1.212925 & 2.564153 \\ \mathrm{H} & -0.904558 & 0.336821 & 2.634838 \\ \mathrm{O} & 2.589802 & 0.261105 & 1.593181 \\ \mathrm{H} & 2.771507 & 0.992354 & 2.213183 \\ \mathrm{H} & 3.031465 & -0.559560 & 1.917789 \\ \mathrm{O} & 1.013063 & 2.721886 & -0.060807 \\ \mathrm{H} & 1.906712 & 3.113208 & -0.156677 \\ \mathrm{H} & 0.616381 & 3.154481 & 0.723814 \\ \mathrm{O} & -1.465667 & 1.630635 & 0.118048 \\ \mathrm{H} & -1.463929 & 2.439847 & -0.428842 \\ \mathrm{H} & -2.386926 & 1.316309 & 0.020737 \\ \mathrm{O} & -2.989167 & -4.116091 & -1.322858 \\ \mathrm{H} & -3.394337 & -4.855607 & -1.796104 \\ \mathrm{H} & -2.961018 & -3.355421 & -1.938130 \\ \mathrm{H} & 2.205291 & -2.245547 & -1.451702 \\ \mathrm{O} & 1.656500 & -1.780431 & -0.801066 \\ & & & \\ \mathrm{C} \mathrm{La}\left(\mathrm{H}{ }_{2} \mathrm{O}\right) 26(\mathrm{OH}) & 2 . & (\mathrm{i}) & \\ \mathrm{E}= & -2499.61717 & & \\ \mathrm{LA} & 0.309809 & -0.172598 & -0.367417 \\ \mathrm{O} & -1.201274 & 1.327998 & -0.332125 \\ \mathrm{H} & -1.668483 & 2.165880 & -0.209508 \\ \mathrm{O} & -2.566241 & 3.862258 & -1.096470 \\ \mathrm{H} & -2.703234 & 4.758123 & -0.756458 \\ \mathrm{O} & -1.822907 & 3.909717 & -1.735829 \\ \mathrm{H} & -0.499473 & 3.746515 & -2.892419 \\ \mathrm{H} & 0.550847 & 3.939881 & -2.603053 \\ \mathrm{H} & 2.338420 & 3.641322 & -2.104331 \\ \mathrm{H} & 2.398288 & -2.077068 \\ \mathrm{H} & & \\ \mathrm{H} & 2.970728 & -2.703383\end{array}$




$\begin{array}{lrrr}\mathrm{O} & 3.009611 & 1.357727 & -3.566928 \\ \mathrm{H} & 3.549626 & 1.215387 & -4.358263 \\ \mathrm{H} & 3.113301 & 0.567685 & -2.980946 \\ \mathrm{O} & 2.770634 & -0.695915 & -1.731179 \\ \mathrm{H} & 3.484237 & -0.524133 & -1.076978 \\ \mathrm{H} & 2.926712 & -1.632722 & -2.033320 \\ \mathrm{O} & 3.196360 & -3.302793 & -2.174298 \\ \mathrm{H} & 3.606973 & -3.813630 & -2.885778 \\ \mathrm{H} & 3.646116 & -3.543322 & -1.337353 \\ \mathrm{O} & 4.212064 & -3.155789 & 0.423369 \\ \mathrm{H} & 4.666188 & -3.798248 & 0.988456 \\ \mathrm{H} & 4.721574 & -2.315400 & 0.460329 \\ \mathrm{O} & 4.919737 & -0.488330 & 0.217933 \\ \mathrm{H} & 5.801217 & -0.179655 & -0.040943 \\ \mathrm{H} & 4.629216 & 0.096099 & 0.959986 \\ \mathrm{O} & 3.940765 & 1.380129 & 2.053161 \\ \mathrm{H} & 4.539310 & 2.064910 & 2.387980 \\ \mathrm{H} & 3.313665 & 1.176952 & 2.799941 \\ \mathrm{O} & 2.005931 & 0.976228 & 3.893433 \\ \mathrm{H} & 2.123471 & 0.506374 & 4.732409 \\ \mathrm{H} & 1.511189 & 1.820757 & 4.090128 \\ \mathrm{O} & 0.467044 & 3.140645 & 4.069059 \\ \mathrm{H} & 0.416942 & 3.903995 & 4.660474 \\ \mathrm{H} & -0.450051 & 2.902036 & 3.807790 \\ \mathrm{O} & -1.883659 & 1.815806 & 3.405909 \\ \mathrm{H} & -1.786418 & -2.926808 & -2.579493 \\ \mathrm{H} & -2.211286 & 1.489580 & 4.257899 \\ \mathrm{H} & -2.701761 & 1.871424 & 2.828379 \\ \mathrm{O} & -4.116391 & 1.552352 & 2.013190 \\ \mathrm{H} & -4.331137 & 1.860914 & 1.111557 \\ \mathrm{H} & -4.183614 & 0.577961 & 1.968115 \\ \mathrm{O} & -4.055277 & -1.310315 & 1.573491 \\ \mathrm{H} & -4.935956 & -1.711099 & 1.641391 \\ \mathrm{H} & -3.906923 & -1.154993 & 0.603608 \\ \mathrm{O} & -3.776819 & -0.672184 & -1.108697 \\ \mathrm{H} & -4.396110 & -1.300526 & -1.553576 \\ \mathrm{H} & -4.134384 & 0.244790 & -1.154367 \\ \mathrm{O} & -4.891465 & -2.940480 & -2.000692 \\ \mathrm{H} & -5.574045 & -3.316040 & -2.573399 \\ \mathrm{H} & -4.073495 & -3.459232 & -2.132527 \\ \mathrm{H} & -5.595762 & 2.006353 & -0.836453 \\ \mathrm{H} & -3.916871 & 2.684264 & -1.067331 \\ \mathrm{H} & -1.74560 & -3.722642 & -2.115904 \\ \mathrm{H} & -40959 & 2.139333\end{array}$




\begin{tabular}{|c|c|c|c|}
\hline $\mathrm{H}$ & 666343 & 181692 & \\
\hline $\mathrm{H}$ & -0.776716 & 0.702684 & \\
\hline $\mathrm{O}$ & 2.102983 & 1.682129 & -0.026042 \\
\hline $\mathrm{H}$ & & & \\
\hline $\mathrm{H}$ & 2.750461 & & \\
\hline $\mathrm{O}$ & -0.833093 & -2.459152 & 0.11 \\
\hline $\mathrm{H}$ & -1.284362 & -3.018213 & -0.5 \\
\hline $\mathrm{H}$ & -1.160800 & -2.7 & 1.0 \\
\hline $\mathrm{O}$ & -1.993389 & -2.8 & \\
\hline $\mathrm{H}$ & -2.380 & -3.7 & \\
\hline $\mathrm{H}$ & -2.75 & -2.2 & \\
\hline $\mathrm{O}$ & $-1.3^{\prime}$ & -1.0 & -2.3 \\
\hline $\mathrm{H}$ & -1.1 & -0.4 & \\
\hline $\mathrm{H}$ & -2.2 & -0.8 & -1. \\
\hline $\mathrm{O}$ & 0.3 & & -2.8 \\
\hline $\mathrm{H}$ & -0.0 & & -2.8 \\
\hline $\mathrm{H}$ & 1.15 & 1.0 & -3.4 \\
\hline $\mathrm{O}$ & 0.7 & -2. & \\
\hline $\mathrm{H}$ & 0.9 & -3. & \\
\hline $\mathrm{H}$ & -0.2 & -2 & \\
\hline $\mathrm{O}$ & & -1.5 & \\
\hline $\mathrm{H}$ & & -2.2 & \\
\hline $\mathrm{H}$ & 1.547 & & \\
\hline \multicolumn{4}{|c|}{$\left[{ }^{1} \mathrm{La}\left(\mathrm{H}_{2} \mathrm{O}\right)_{9}\right]^{3+}$} \\
\hline \multicolumn{4}{|c|}{$E=-1123.1190831$} \\
\hline LA & 0.000000 & & \\
\hline $\mathrm{O}$ & -1.225 & & \\
\hline $\mathrm{H}$ & -0.8 & & \\
\hline $\mathrm{O}$ & -2.4 & 0.4 & -0.5 \\
\hline $\mathrm{H}$ & $-3.1 \mathrm{C}$ & -0.1 & -0.7 \\
\hline $\mathrm{O}$ & -1.73 & -1. & \\
\hline $\mathrm{H}$ & -2.0 & -2.3 & \\
\hline $\mathrm{O}$ & 0.00 & -2.4 & \\
\hline $\mathrm{H}$ & -0.2 & -2.7 & -1.8 \\
\hline $\mathrm{O}$ & & -1.4 & \\
\hline $\mathrm{H}$ & 2.15 & -1.7 & \\
\hline $\mathrm{O}$ & 1.739422 & 1.44 & 1.34 \\
\hline $\mathrm{H}$ & 2.01 & 2.3 & \\
\hline $\mathrm{O}$ & 2.413513 & -0.448854 & -0.9 \\
\hline $\mathrm{H}$ & 2.736738 & -1.2 & \\
\hline $\mathrm{O}$ & 0.000000 & & -2.6 \\
\hline $\mathrm{H}$ & -0.773131 & 0.05 & -3.2 \\
\hline $\mathrm{O}$ & 0.000000 & 2.451231 & -0.907297 \\
\hline $\mathrm{H}$ & -0.283012 & 3.263438 & -0.451029 \\
\hline $\mathrm{H}$ & -2.736738 & 1.229405 & -1.399797 \\
\hline
\end{tabular}




$\begin{array}{rrrr}\mathrm{H} & -2.254814 & -1.157548 & 2.119359 \\ \mathrm{H} & 0.283012 & -3.263438 & -0.451029 \\ \mathrm{H} & 3.192734 & 0.123469 & -0.798489 \\ \mathrm{H} & 0.817094 & -1.894237 & 2.567978 \\ \mathrm{H} & -2.158648 & 1.747759 & 1.806119 \\ \mathrm{H} & 0.257062 & 2.733377 & -1.803250 \\ \mathrm{H} & 2.254814 & 1.157548 & 2.119359 \\ \mathrm{H} & 0.773131 & -0.054757 & -3.221209\end{array}$

\begin{tabular}{cccc}
\multicolumn{5}{c}{$\left[{ }^{1} \mathrm{La}\left(\mathrm{H}_{2} \mathrm{O}\right)_{8}(\mathrm{OH})\right]^{2+}(\mathrm{a})$} \\
$\mathrm{E}=-1122.97402$ & & \\
$\mathrm{O}$ & 1.991141 & 1.038456 & -1.391649 \\
$\mathrm{H}$ & 2.924908 & 1.099651 & -1.134398 \\
$\mathrm{H}$ & 1.845843 & 1.705542 & -2.081580 \\
$\mathrm{O}$ & -0.777147 & 1.958978 & -1.587677 \\
$\mathrm{H}$ & -1.378311 & 1.770126 & -2.326199 \\
$\mathrm{H}$ & -0.898658 & 2.895895 & -1.365993 \\
$\mathrm{O}$ & -1.747222 & -0.803224 & -1.808236 \\
$\mathrm{H}$ & -2.689984 & -0.904350 & -1.599103 \\
$\mathrm{H}$ & -1.567643 & -1.388971 & -2.560645 \\
$\mathrm{O}$ & 1.143736 & -1.763286 & -1.595889 \\
$\mathrm{H}$ & 1.780366 & -1.511861 & -2.283803 \\
$\mathrm{H}$ & 1.165200 & -2.731359 & -1.536087 \\
$\mathrm{O}$ & 0.593829 & 2.366942 & 1.110723 \\
$\mathrm{H}$ & 1.201045 & 3.103835 & 0.943321 \\
$\mathrm{H}$ & 0.368822 & 2.400052 & 2.055058 \\
$\mathrm{O}$ & -0.683571 & -2.497674 & 0.702972 \\
$\mathrm{H}$ & -1.178609 & -3.270959 & 0.392696 \\
$\mathrm{H}$ & -0.747416 & -2.492342 & 1.673345 \\
$\mathrm{O}$ & -2.543806 & 0.567988 & 0.686791 \\
$\mathrm{H}$ & -2.556222 & 0.414728 & 1.647873 \\
$\mathrm{H}$ & -3.275033 & 1.171782 & 0.486056 \\
$\mathrm{O}$ & 2.386957 & -0.685601 & 1.036600 \\
$\mathrm{H}$ & 2.495446 & -0.620994 & 1.999376 \\
$\mathrm{H}$ & 3.021477 & -1.355660 & 0.738793 \\
$\mathrm{LA}$ & -0.008664 & -0.006709 & 0.113539 \\
$\mathrm{O}$ & -0.327577 & -0.146069 & 2.254025 \\
$\mathrm{H}$ & -0.308126 & -0.194797 & 3.218288
\end{tabular}

$\left[{ }^{1} \mathrm{La}\left(\mathrm{H}_{2} \mathrm{O}\right)_{8}(\mathrm{OH})\right]^{2+}(\mathrm{b})$

$\mathrm{E}=-1122.973975$

$\begin{array}{rrrr}\mathrm{O} & 2.495030 & 0.853826 & -0.430422 \\ \mathrm{H} & 2.763081 & 1.491497 & -1.111342 \\ \mathrm{H} & 3.134759 & 0.124093 & -0.488488 \\ \mathrm{O} & 1.745856 & -1.976515 & -0.751608 \\ \mathrm{H} & 2.188148 & -2.800013 & -0.494951\end{array}$




\begin{tabular}{|c|c|c|c|}
\hline $\mathrm{H}$ & & & \\
\hline $\mathrm{O}$ & & & \\
\hline $\mathrm{H}$ & 1.421590 & -1.910714 & \\
\hline $\mathrm{H}$ & & & \\
\hline $\mathrm{O}$ & & & \\
\hline $\mathrm{H}$ & 1.721899 & & \\
\hline $\mathrm{H}$ & & 2.405304 & \\
\hline $\mathrm{O}$ & -1.707185 & & \\
\hline $\mathrm{H}$ & -1.676650 & -0.04 & 870 \\
\hline $\mathrm{H}$ & -2.612 & 0.4 & 61 \\
\hline $\mathrm{O}$ & -2.637 & & \\
\hline $\mathrm{H}$ & -2.867 & 1.4 & \\
\hline $\mathrm{H}$ & -3.20 & & \\
\hline $\mathrm{O}$ & -0.19 & & \\
\hline $\mathrm{H}$ & -0.2 & & \\
\hline $\mathrm{H}$ & -0.1 & & \\
\hline $\mathrm{O}$ & & & \\
\hline $\mathrm{H}$ & -1.9 & -2.6 & \\
\hline $\mathrm{H}$ & -1.50 & -2.8 & -0 \\
\hline LA & -0.0 & & \\
\hline $\mathrm{O}$ & -0.08 & -0.4 & -2 \\
\hline $\mathrm{H}$ & -0.2 & & \\
\hline \multicolumn{4}{|c|}{$\left[{ }^{1} \mathrm{La}\left(\mathrm{H}_{2} \mathrm{O}\right)_{8}(\mathrm{OH})\right]^{2+}(\mathrm{c})$} \\
\hline \multicolumn{4}{|c|}{$\mathrm{E}=-1122.974013$} \\
\hline $\mathrm{O}$ & -2.044946 & & \\
\hline $\mathrm{H}$ & -2.5 & & \\
\hline I & -2.09 & -1. & \\
\hline $\mathrm{O}$ & & $-0 .($ & -2 \\
\hline & 0.12 & -0.1 & \\
\hline & & & -0 \\
\hline $\mathrm{O}$ & 1.48 & -2.1 & \\
\hline $\mathrm{H}$ & & & \\
\hline $\mathrm{H}$ & & -2.1 & \\
\hline $\mathrm{O}$ & -1.4 & & \\
\hline $\mathrm{H}$ & -2.24 & & \\
\hline $\mathrm{H}$ & -1.33 & & \\
\hline $\mathrm{O}$ & 2.101 & 1.4 & -0.8 \\
\hline $\mathrm{H}$ & 2.59 & 2.2 & \\
\hline $\mathrm{H}$ & 2.170423 & 1.296803 & -1.83 \\
\hline $\mathrm{O}$ & -0.302 & -2.007692 & \\
\hline $\mathrm{H}$ & -0.049257 & -2.935335 & 3063 \\
\hline $\mathrm{H}$ & -0.973952 & -1.999219 & 2.379809 \\
\hline $\mathrm{O}$ & -2.119704 & & \\
\hline $\mathrm{H}$ & -3.045354 & 0.072199 & 1.382858 \\
\hline $\mathrm{H}$ & -2.128418 & 1.004825 & 2.227457 \\
\hline
\end{tabular}




$\begin{array}{rrrr}\mathrm{O} & 0.167007 & 2.117044 & 1.568243 \\ \mathrm{H} & -0.044850 & 3.040174 & 1.355787 \\ \mathrm{H} & 0.838526 & 2.141910 & 2.269157 \\ \mathrm{O} & 2.020719 & -0.145987 & 1.695257 \\ \mathrm{H} & 2.944398 & 0.072578 & 1.492670 \\ \mathrm{H} & 2.042400 & -0.784791 & 2.425657\end{array}$

$$
\begin{array}{cccc}
{\left[{ }^{1} \mathrm{La}\left(\mathrm{H}_{2} \mathrm{O}\right)_{8}(\mathrm{OH})\right]^{2+}(\mathrm{d})} & \\
\mathrm{E}=-1122.973977 & & \\
\mathrm{O} & -1.707221 & 0.148747 & 1.983808 \\
\mathrm{H} & -1.676026 & -0.051715 & 2.931686 \\
\mathrm{H} & -2.613476 & 0.429556 & 1.774795 \\
\mathrm{O} & -2.638433 & 0.639627 & -0.553417 \\
\mathrm{H} & -2.869010 & 1.477808 & -0.985516 \\
\mathrm{H} & -3.201050 & -0.032495 & -0.971691 \\
\mathrm{O} & -0.204510 & 2.371735 & -1.239965 \\
\mathrm{H} & -0.228950 & 2.340178 & -2.210435 \\
\mathrm{H} & -0.141570 & 3.306225 & -0.988271 \\
\mathrm{O} & 0.838637 & 1.852425 & 1.694140 \\
\mathrm{H} & 0.373943 & 2.402114 & 2.344722 \\
\mathrm{H} & 1.715355 & 2.250207 & 1.565490 \\
\mathrm{O} & -1.483772 & -2.230128 & 0.007253 \\
\mathrm{H} & -1.964113 & -2.639150 & 0.744058 \\
\mathrm{H} & -1.502154 & -2.868954 & -0.723443 \\
\mathrm{O} & 1.750365 & -1.971482 & -0.754397 \\
\mathrm{H} & 2.194481 & -2.794185 & -0.498320 \\
\mathrm{H} & 1.568388 & -2.022600 & -1.709688 \\
\mathrm{O} & 1.149220 & -1.034138 & 2.107378 \\
\mathrm{H} & 1.426075 & -1.910795 & 2.415955 \\
\mathrm{H} & 1.513352 & -0.393332 & 2.738808 \\
\mathrm{O} & 2.493020 & 0.859277 & -0.429514 \\
\mathrm{H} & 2.760748 & 1.499283 & -1.108362 \\
\mathrm{H} & 3.133776 & 0.130569 & -0.489196 \\
\mathrm{LA} & -0.020119 & -0.035232 & -0.113932 \\
\mathrm{O} & -0.082197 & -0.454145 & -2.213027 \\
\mathrm{H} & -0.263843 & -0.569826 & -3.154555 \\
& & & \\
{\left[{ }^{1} \mathrm{La}\left(\mathrm{H}_{2} \mathrm{O}\right)_{8}(\mathrm{OH})\right]^{2+}(\mathrm{e})} & \\
\mathrm{E}=-1122.973976 & & \\
\mathrm{O} & -1.486987 & -2.228148 & 0.011178 \\
\mathrm{H} & -1.966204 & -2.635835 & 0.749459 \\
\mathrm{H} & -1.506136 & -2.868436 & -0.718217 \\
\mathrm{O} & 1.747766 & -1.974091 & -0.752962 \\
\mathrm{H} & 1.564566 & -2.026046 & -1.707982 \\
\mathrm{H} & 2.192041 & -2.796572 & -0.496474 \\
\mathrm{O} & 1.149450 & -1.032857 & 2.107941
\end{array}
$$




$\begin{array}{lccc}\mathrm{H} & 1.425692 & -1.909258 & 2.417789 \\ \mathrm{H} & 1.514845 & -0.391269 & 2.737855 \\ \mathrm{O} & 0.843113 & 1.852946 & 1.691827 \\ \mathrm{H} & 0.379401 & 2.404546 & 2.341493 \\ \mathrm{H} & 1.719968 & 2.249837 & 1.561365 \\ \mathrm{O} & -1.705278 & 0.154842 & 1.984932 \\ \mathrm{H} & -1.673305 & -0.043770 & 2.933178 \\ \mathrm{H} & -2.611427 & 0.436376 & 1.776425 \\ \mathrm{O} & 2.493199 & 0.856818 & -0.432851 \\ \mathrm{H} & 2.761001 & 1.495743 & -1.112685 \\ \mathrm{H} & 3.132889 & 0.127165 & -0.492520 \\ \mathrm{O} & -2.637939 & 0.640830 & -0.553069 \\ \mathrm{H} & -3.201079 & -0.032600 & -0.968529 \\ \mathrm{H} & -2.869125 & 1.477706 & -0.987353 \\ \mathrm{O} & -0.203442 & 2.370202 & -1.243269 \\ \mathrm{H} & -0.228377 & 2.337432 & -2.213683 \\ \mathrm{H} & -0.140024 & 3.305002 & -0.992841 \\ \mathrm{LA} & -0.020322 & -0.035433 & -0.113785 \\ \mathrm{O} & -0.083656 & -0.457536 & -2.212235 \\ \mathrm{H} & -0.266181 & -0.574416 & -3.153445\end{array}$

$\left[{ }^{1} \mathrm{La}\left(\mathrm{H}_{2} \mathrm{O}\right)_{8}(\mathrm{OH})\right]^{2+}(\mathrm{f})$

$\mathrm{E}=-1122.973975$

$\begin{array}{lrrr}\mathrm{O} & 1.145850 & -1.033976 & 2.108694 \\ \mathrm{H} & 1.421590 & -1.910714 & 2.418038 \\ \mathrm{H} & 1.510633 & -0.393033 & 2.739625 \\ \mathrm{O} & 0.844157 & 1.852122 & 1.692357 \\ \mathrm{H} & 1.721899 & 2.247030 & 1.561758 \\ \mathrm{H} & 0.381435 & 2.405304 & 2.341378 \\ \mathrm{O} & 2.495030 & 0.853826 & -0.430422 \\ \mathrm{H} & 2.763081 & 1.491497 & -1.111342 \\ \mathrm{H} & 3.134759 & 0.124093 & -0.488488 \\ \mathrm{O} & 1.745856 & -1.976515 & -0.751608 \\ \mathrm{H} & 2.188148 & -2.800013 & -0.494951 \\ \mathrm{H} & 1.563808 & -2.027804 & -1.706889 \\ \mathrm{O} & -1.707185 & 0.157477 & 1.983370 \\ \mathrm{H} & -1.676650 & -0.040153 & 2.931870 \\ \mathrm{H} & -2.612606 & 0.440380 & 1.773561 \\ \mathrm{O} & -2.637175 & 0.643308 & -0.555276 \\ \mathrm{H} & -3.200864 & -0.029725 & -0.970640 \\ \mathrm{H} & -2.867428 & 1.480190 & -0.990051 \\ \mathrm{O} & -1.489514 & -2.226362 & 0.011251 \\ \mathrm{H} & -1.970239 & -2.632084 & 0.749638 \\ \mathrm{H} & -1.509408 & -2.867586 & -0.717303 \\ \mathrm{O} & -0.198711 & 2.370080 & -1.243357 \\ \mathrm{H} & -0.223386 & 2.337448 & -2.213781\end{array}$




\begin{tabular}{|c|c|c|c|}
\hline $\mathrm{H}$ & -0.135562 & 3.304837 & -0.992703 \\
\hline LA & -0.020203 & -0.035430 & -0.113963 \\
\hline $\mathrm{O}$ & -0.082408 & -0.457034 & -2.212516 \\
\hline $\mathrm{H}$ & -0.264872 & -0.573582 & -3.153781 \\
\hline \multicolumn{4}{|c|}{$\left[{ }^{1} \mathrm{La}\left(\mathrm{H}_{2} \mathrm{O}\right)_{8}(\mathrm{OH})\right]^{2+}(\mathrm{g})$} \\
\hline \multicolumn{4}{|c|}{$\mathrm{E}=-1122.974121$} \\
\hline $\mathrm{O}$ & 1.953312 & 1.562777 & -0.982786 \\
\hline $\mathrm{H}$ & 2.009800 & 1.482767 & -1.949429 \\
\hline $\mathrm{H}$ & 2.395447 & 2.392934 & -0.747290 \\
\hline $\mathrm{O}$ & 0.155865 & 2.101039 & 1.589458 \\
\hline $\mathrm{H}$ & -0.131438 & 3.014000 & 1.429544 \\
\hline $\mathrm{H}$ & 0.849502 & 2.143710 & 2.267562 \\
\hline $\mathrm{O}$ & -1.605165 & 1.960046 & -0.900554 \\
\hline $\mathrm{H}$ & -2.411500 & 2.420498 & -0.622377 \\
\hline $\mathrm{H}$ & -1.579445 & 2.006914 & -1.870625 \\
\hline $\mathrm{O}$ & 2.112964 & -0.062185 & 1.582797 \\
\hline $\mathrm{H}$ & 3.023243 & 0.207208 & 1.380630 \\
\hline $\mathrm{H}$ & 2.171765 & -0.726482 & 2.288258 \\
\hline $\mathrm{O}$ & -0.048820 & -2.037617 & 1.673569 \\
\hline $\mathrm{H}$ & 0.223585 & -2.951209 & 1.492028 \\
\hline $\mathrm{H}$ & -0.703432 & -2.080775 & 2.388996 \\
\hline $\mathrm{O}$ & 1.562722 & -1.995357 & -0.891901 \\
\hline $\mathrm{H}$ & 2.390125 & -2.440270 & -0.653043 \\
\hline $\mathrm{H}$ & 1.470544 & -2.076362 & -1.855888 \\
\hline $\mathrm{O}$ & -2.033715 & 0.142799 & 1.675164 \\
\hline $\mathrm{H}$ & -2.950257 & -0.140143 & 1.528100 \\
\hline $\mathrm{H}$ & -2.060233 & 0.817020 & 2.373010 \\
\hline $\mathrm{O}$ & -2.000559 & -1.601356 & -0.810509 \\
\hline $\mathrm{H}$ & -2.068604 & -1.562526 & -1.778997 \\
\hline $\mathrm{H}$ & -2.457571 & -2.409163 & -0.531242 \\
\hline LA & -0.005521 & -0.000543 & -0.124399 \\
\hline $\mathrm{O}$ & -0.067815 & -0.068812 & -2.285003 \\
\hline $\mathrm{H}$ & -0.087143 & -0.077848 & -3.250399 \\
\hline
\end{tabular}

\begin{tabular}{|c|c|c|c|}
\hline \multicolumn{4}{|c|}{$\left[{ }^{1} \mathrm{La}\left(\mathrm{H}_{2} \mathrm{O}\right)_{8}(\mathrm{OH})\right]^{2+}(\mathrm{h})$} \\
\hline $\mathrm{O}$ & -0.204510 & 2.371735 & -1.239965 \\
\hline $\mathrm{H}$ & -0.228950 & 2.340178 & 10435 \\
\hline $\mathrm{H}$ & $-0.1<$ & 3.306225 & 8271 \\
\hline $\mathrm{O}$ & 0.8 & 1.85 & \\
\hline $\mathrm{H}$ & 0.373943 & 2.40 & 722 \\
\hline $\mathrm{H}$ & 1.715355 & 2.25 & 5490 \\
\hline $\mathrm{O}$ & -1.707221 & 0.148747 & 1.983808 \\
\hline $\mathrm{H}$ & -1.676026 & -0.051715 & 2.931686 \\
\hline $\mathrm{H}$ & -2.613476 & 0.429556 & 1.774795 \\
\hline
\end{tabular}




\begin{tabular}{|c|c|c|c|}
\hline $\mathrm{O}$ & 2.638433 & 0.639627 & \\
\hline $\mathrm{H}$ & 2.869010 & 1.477808 & \\
\hline $\mathrm{H}$ & -3.201050 & -0.032495 & -0.971691 \\
\hline $\mathrm{O}$ & 2.493020 & 0.859277 & \\
\hline $\mathrm{H}$ & 2.760748 & & \\
\hline $\mathrm{H}$ & 3.133776 & & -0.4 \\
\hline $\mathrm{O}$ & 1.75 & & \\
\hline $\mathrm{H}$ & 1.56 & -2.0 & \\
\hline $\mathrm{H}$ & & & \\
\hline $\mathrm{O}$ & 220 & -1.0 & \\
\hline $\mathrm{H}$ & & & \\
\hline $\mathrm{H}$ & 1.5 & -0.3 & \\
\hline $\mathrm{O}$ & -1.4 & -2.2 & \\
\hline $\mathrm{H}$ & -1.9 & -2.6 & 58 \\
\hline $\mathrm{H}$ & -1.50 & -2.8 & -0 . \\
\hline LA & -0.02 & & -0 \\
\hline $\mathrm{O}$ & -0.08 & -0.4 & -2.2 \\
\hline $\mathrm{H}$ & -0.2 & -0.5 & -3 \\
\hline \multicolumn{4}{|c|}{$\left[{ }^{1} \mathrm{La}\left(\mathrm{H}_{2} \mathrm{O}\right)_{8}(\mathrm{OH})\right]^{2+}(\mathrm{i})$} \\
\hline \multicolumn{4}{|c|}{$E=-1122.973976$} \\
\hline $\mathrm{O}$ & & & \\
\hline $\mathrm{H}$ & 3.2 & & -0 . \\
\hline $\mathrm{H}$ & $2.8^{\prime}$ & -1.4 & \\
\hline $\mathrm{O}$ & & -0.1 & \\
\hline $\mathrm{H}$ & $1.6^{\circ}$ & 0.0 & \\
\hline $\mathrm{H}$ & 2.6 & -0.4 & \\
\hline $\mathrm{O}$ & 0.20 & -2.3 & -1. \\
\hline $\mathrm{H}$ & 0.1 & -3.3 & -0 . \\
\hline $\mathrm{H}$ & & -2.3 & -2 \\
\hline $\mathrm{O}$ & & & -2.2 \\
\hline $\mathrm{H}$ & 0.27 & 0.5 & -3 \\
\hline LA & & & \\
\hline $\mathrm{O}$ & $-1.74^{\prime}$ & & -0 . \\
\hline $\mathrm{H}$ & -2.19 & 2.7 & -0 . \\
\hline $\mathrm{H}$ & -1.56 & 2.02 & -1. \\
\hline $\mathrm{O}$ & 1.486 & 2.2 & \\
\hline $\mathrm{H}$ & 1.506 & 2.86 & -0.7 \\
\hline $\mathrm{H}$ & 1.964 & 2.6 & \\
\hline $\mathrm{O}$ & -2.492953 & & \\
\hline $\mathrm{H}$ & -2.759538 & 4739 & -1 . \\
\hline $\mathrm{H}$ & -3.133312 & -0.12 & -0.4 \\
\hline $\mathrm{O}$ & -1.152085 & 1.031127 & \\
\hline $\mathrm{H}$ & -1.428465 & 1.906804 & \\
\hline $\mathrm{H}$ & -1.516671 & 0.388095 & 2.735861 \\
\hline $\mathrm{O}$ & -0.847409 & -1.853416 & 1.689723 \\
\hline
\end{tabular}




$$
\begin{array}{llll}
\mathrm{H} & -0.385589 & -2.407115 & 2.338957 \\
\mathrm{H} & -1.724318 & -2.249242 & 1.556349
\end{array}
$$

Geometries of calculated acidities of glycine $\left[\mathrm{Met}(\mathrm{III})\left(\mathrm{H}_{2} \mathrm{O}\right)_{\mathrm{z}-2}\right]^{3+} \operatorname{complex}($ Table 6).

$$
\begin{array}{cccc}
{\left[\left(\mathrm{NH}_{3}{ }^{+} \mathrm{CH}_{2} \mathrm{COO}^{-}\right)^{1} \mathrm{Al}\left(\mathrm{H}_{2} \mathrm{O}\right)_{4}\right]^{3}} \\
\mathrm{E}=-831.9250302 & & \\
\mathrm{C} & -2.812072 & -0.000068 & 0.641655 \\
\mathrm{H} & -3.047395 & 0.881600 & 1.246285 \\
\mathrm{H} & -3.047403 & -0.881866 & 1.246094 \\
\mathrm{C} & -1.327316 & -0.000051 & 0.325086 \\
\mathrm{O} & -0.472210 & -0.000058 & 1.273087 \\
\mathrm{O} & -0.837205 & -0.000021 & -0.866383 \\
\mathrm{AL} & 0.945071 & -0.000001 & -0.032655 \\
\mathrm{~N} & -3.700843 & 0.000064 & -0.580435 \\
\mathrm{H} & -3.563560 & -0.828990 & -1.174168 \\
\mathrm{H} & -4.691192 & -0.000059 & -0.289310 \\
\mathrm{O} & 1.058291 & 1.960894 & -0.148445 \\
\mathrm{O} & 1.058366 & -1.960874 & -0.148612 \\
\mathrm{O} & 2.171396 & 0.000102 & -1.508667 \\
\mathrm{H} & 2.503721 & 0.790362 & -1.978677 \\
\mathrm{O} & 2.273719 & -0.000037 & 1.358520 \\
\mathrm{H} & 3.248310 & 0.000012 & 1.274156 \\
\mathrm{H} & 2.049595 & -0.000097 & 2.312301 \\
\mathrm{H} & 0.550929 & 2.531910 & -0.756984 \\
\mathrm{H} & 1.471636 & 2.541672 & 0.520770 \\
\mathrm{H} & 2.503692 & -0.790094 & -1.978806 \\
\mathrm{H} & 1.471848 & -2.541690 & 0.520487 \\
\mathrm{H} & 0.550957 & -2.531865 & -0.757137 \\
\mathrm{H} & -3.563692 & 0.829336 & -1.173893 \\
& & & \\
{[(\mathrm{NH}} & + \\
\mathrm{E}= & -831.8179191 & & \\
\mathrm{AL} & 0.951081 & 0.114060 & 0.121075 \\
\mathrm{O} & 2.090581 & 1.378817 & -0.806590 \\
\mathrm{O} & 2.371724 & -1.126661 & 0.726604 \\
\mathrm{H} & 2.353654 & 1.531246 & -1.727696 \\
\mathrm{H} & 2.196387 & 2.187989 & -0.268525 \\
\mathrm{O} & 1.161167 & -0.978039 & -1.574066 \\
\mathrm{O} & -0.495880 & -1.088302 & 0.746725 \\
\mathrm{O} & 1.060336 & 1.267084 & 1.403391 \\
\mathrm{O} & -0.903704 & 0.546954 & -0.647893 \\
\mathrm{H} & 2.124438 & -1.932058 & 1.217027 \\
\mathrm{H} & 3.097921 & -0.703863 & 1.222560 \\
& 1.858498 & -1.642608 & -1.709023 \\
& 0.570476 & -0.977532 & -2.344590
\end{array}
$$
$\begin{array}{llll}\text { AL } & 0.951081 & 0.114060 & 0.121075\end{array}$
$\begin{array}{llll}\mathrm{O} & 2.090581 & 1.378817 & -0.806590\end{array}$
$\begin{array}{llll}\mathrm{O} & 2.371724 & -1.126661 & 0.726604\end{array}$
H $\quad 2.353654 \quad 1.531246-1.727696$
H $\quad 2.196387 \quad 2.187989-0.268525$
O $\quad 1.161167 \quad-0.978039 \quad-1.574066$
$\begin{array}{llll}\mathrm{O} & -0.495880 & -1.088302 & 0.746725\end{array}$
$\begin{array}{llll}\text { O } & 1.060336 & 1.267084 & 1.403391\end{array}$
O $\quad-0.903704 \quad 0.546954-0.647893$
H $\quad 2.124438-1.932058 \quad 1.217027$
$\begin{array}{llll}\mathrm{H} & 3.097921 & -0.703863 & 1.222560\end{array}$
H $\quad \begin{array}{llll}1.858498 & -1.642608 & -1.709023\end{array}$
H $\quad 0.570476 \quad-0.977532 \quad-2.344590$ 


\begin{tabular}{|c|c|c|c|}
\hline $\mathrm{C}$ & -1.338021 & -0.376016 & 0.120478 \\
\hline $\mathrm{H}$ & 0.806095 & 1.217347 & 2.329083 \\
\hline $\mathrm{C}$ & -2.831386 & -0.601813 & 0.289955 \\
\hline $\mathrm{N}$ & -3.587692 & 0.604883 & -0.221631 \\
\hline $\mathrm{H}$ & -3.082163 & -0.767606 & 1.338632 \\
\hline $\mathrm{H}$ & -3.141213 & -1.476620 & -0.287405 \\
\hline $\mathrm{H}$ & -3.165185 & 0.943096 & -1.097555 \\
\hline $\mathrm{H}$ & -4.577705 & 0.386835 & -0.388983 \\
\hline $\mathrm{H}$ & -3.548768 & 1.384957 & 0.445961 \\
\hline \multicolumn{4}{|c|}{$\begin{array}{l}{\left[\left(\mathrm{NH}_{3}{ }^{+} \mathrm{CH}_{2} \mathrm{COO}^{-}\right){ }^{1} \mathrm{Al}\left(\mathrm{H}_{2} \mathrm{O}\right)_{3}\left(\mathrm{OH}^{-}\right)\right]^{2+}(\mathrm{b})} \\
\mathrm{E}=-831.8141351\end{array}$} \\
\hline $\mathrm{AL}$ & -1.053535 & -0.004097 & -0.093093 \\
\hline $\mathrm{O}$ & -1.873171 & 0.158626 & 1.690391 \\
\hline $\mathrm{O}$ & -1.115513 & -1.969392 & 0.204248 \\
\hline $\mathrm{H}$ & -2.320366 & -0.592231 & 2.117111 \\
\hline $\mathrm{H}$ & -2.320484 & 0.975609 & 1.970380 \\
\hline $\mathrm{O}$ & -1.090146 & 1.983068 & -0.138493 \\
\hline $\mathrm{O}$ & -2.337774 & -0.087432 & -1.220556 \\
\hline $\mathrm{O}$ & 0.480371 & -0.122191 & -1.340573 \\
\hline $\mathrm{O}$ & 0.823237 & 0.050925 & 0.806349 \\
\hline $\mathrm{H}$ & -1.636977 & -2.396081 & -0.502501 \\
\hline $\mathrm{H}$ & -0.528021 & -2.625169 & 0.613815 \\
\hline $\mathrm{H}$ & -1.608678 & 2.279568 & -0.911199 \\
\hline $\mathrm{H}$ & -0.491809 & 2.694023 & 0.142784 \\
\hline $\mathrm{H}$ & -3.297677 & -0.092906 & -1.178219 \\
\hline $\mathrm{C}$ & 1.295418 & -0.059406 & -0.380141 \\
\hline $\mathrm{C}$ & 2.792062 & -0.153717 & -0.629503 \\
\hline $\mathrm{N}$ & 3.559583 & 0.138383 & 0.642793 \\
\hline $\mathrm{H}$ & 3.102730 & 0.542181 & -1.410624 \\
\hline $\mathrm{H}$ & 3.036232 & -1.167282 & -0.957084 \\
\hline $\mathrm{H}$ & 3.055855 & -0.243903 & 1.455677 \\
\hline $\mathrm{H}$ & 4.503692 & -0.266443 & 0.613965 \\
\hline $\mathrm{H}$ & 3.663464 & 1.147111 & 0.803484 \\
\hline \multicolumn{4}{|c|}{$\begin{array}{l}{\left[\left(\mathrm{NH}_{3}{ }^{+} \mathrm{CH}_{2} \mathrm{COO}^{-}\right){ }^{1} \mathrm{Al}\left(\mathrm{H}_{2} \mathrm{O}\right)_{3}\left(\mathrm{OH}^{-}\right)\right]^{2+}(\mathrm{c})} \\
\mathrm{E}=-831.8247049\end{array}$} \\
\hline $\mathrm{AL}$ & 1.013308 & -0.000041 & 0.144000 \\
\hline $\mathrm{O}$ & 2.444157 & 0.000520 & -1.186819 \\
\hline $\mathrm{O}$ & 0.944589 & 1.997406 & 0.111225 \\
\hline $\mathrm{H}$ & 3.360065 & 0.000468 & -0.854442 \\
\hline $\mathrm{H}$ & 2.440379 & 0.000906 & -2.157799 \\
\hline $\mathrm{O}$ & 0.945348 & -1.997408 & 0.110484 \\
\hline $\mathrm{O}$ & 2.125309 & -0.000186 & 1.451384 \\
\hline $\mathrm{O}$ & -0.378122 & 0.000246 & -1.227342 \\
\hline $\mathrm{H}$ & 1.536390 & 2.393343 & 0.776725 \\
\hline
\end{tabular}




\begin{tabular}{|c|c|c|c|}
\hline $\mathrm{H}$ & 0.894814 & 2.594619 & -0.653487 \\
\hline $\mathrm{H}$ & 1.537371 & -2.393009 & 0.775991 \\
\hline $\mathrm{H}$ & 0.895639 & -2.594770 & -0.654114 \\
\hline $\mathrm{H}$ & 2.024023 & -0.000487 & 2.407943 \\
\hline $\mathrm{C}$ & -1.289309 & -0.000071 & -0.327215 \\
\hline $\mathrm{C}$ & -2.756463 & -0.000096 & -0.717380 \\
\hline $\mathrm{O}$ & -0.936158 & -0.000428 & 0.886234 \\
\hline $\mathrm{N}$ & -3.614010 & -0.000043 & 0.527962 \\
\hline $\mathrm{H}$ & -2.995805 & 0.886332 & -1.309046 \\
\hline $\mathrm{H}$ & -2.995729 & -0.886672 & -1.308862 \\
\hline $\mathrm{H}$ & -4.613821 & -0.000519 & 0.289494 \\
\hline $\mathrm{H}$ & -3.421957 & -0.822837 & 1.113308 \\
\hline $\mathrm{H}$ & -3.422661 & 0.823270 & 1.112799 \\
\hline \multicolumn{4}{|c|}{$\begin{array}{l}{\left[\left(\mathrm{NH}_{3}{ }^{+} \mathrm{CH}_{2} \mathrm{COO}^{-}\right){ }^{1} \mathrm{Al}\left(\mathrm{H}_{2} \mathrm{O}\right)_{3}\left(\mathrm{OH}^{-}\right)\right]^{2+}(\mathrm{d})} \\
\mathrm{E}=-831.817919\end{array}$} \\
\hline $\mathrm{AL}$ & -0.950966 & 0.114113 & 0.121068 \\
\hline $\mathrm{O}$ & -2.090658 & 1.377363 & -0.808643 \\
\hline $\mathrm{O}$ & -2.371823 & -1.125597 & 0.728131 \\
\hline $\mathrm{H}$ & -2.197543 & 2.187347 & -0.272065 \\
\hline $\mathrm{H}$ & -2.358494 & 1.526139 & -1.728951 \\
\hline $\mathrm{O}$ & -1.160733 & -0.980227 & -1.572764 \\
\hline $\mathrm{O}$ & -1.061066 & 1.268987 & 1.401617 \\
\hline $\mathrm{O}$ & 0.495932 & -1.086996 & 0.748238 \\
\hline $\mathrm{O}$ & 0.903907 & 0.546352 & -0.648530 \\
\hline $\mathrm{H}$ & -2.125004 & -1.931094 & 1.218616 \\
\hline $\mathrm{H}$ & -3.097146 & -0.701860 & 1.224569 \\
\hline $\mathrm{H}$ & -1.857918 & -1.645018 & -1.707340 \\
\hline $\mathrm{H}$ & -0.569865 & -0.980198 & -2.343154 \\
\hline $\mathrm{H}$ & -0.804539 & 1.221577 & 2.326799 \\
\hline $\mathrm{C}$ & 1.338168 & -0.375622 & 0.120979 \\
\hline $\mathrm{C}$ & 2.831519 & -0.601507 & 0.290532 \\
\hline $\mathrm{N}$ & 3.587851 & 0.604684 & -0.222225 \\
\hline $\mathrm{H}$ & 3.141068 & -1.476799 & -0.286247 \\
\hline $\mathrm{H}$ & 3.082434 & -0.766572 & 1.339282 \\
\hline $\mathrm{H}$ & 3.550638 & 1.384762 & 0.445456 \\
\hline $\mathrm{H}$ & 4.577429 & 0.386023 & -0.391320 \\
\hline $\mathrm{H}$ & 3.163947 & 0.943151 & -1.097406 \\
\hline \multicolumn{4}{|c|}{$\left[\left(\mathrm{NH}_{2} \mathrm{CH}_{2} \mathrm{COO}^{-}\right){ }^{1} \mathrm{Al}\left(\mathrm{H}_{2} \mathrm{O}\right)_{3}(\mathrm{OH})\right]^{+}(\mathrm{a})$} \\
\hline \multicolumn{4}{|c|}{$E=-831.5655979$} \\
\hline $\mathrm{AL}$ & -0.869352 & 0.140089 & 0.100054 \\
\hline $\mathrm{O}$ & -2.064970 & -0.737210 & 1.389792 \\
\hline $\mathrm{O}$ & -2.290203 & 0.445900 & -1.280980 \\
\hline $\mathrm{H}$ & -2.224032 & -0.078995 & 2.093926 \\
\hline $\mathrm{H}$ & -1.978723 & -1.626982 & 1.764428 \\
\hline
\end{tabular}




$\begin{array}{cccc}\mathrm{O} & -1.385718 & 1.580056 & 0.957659 \\ \mathrm{O} & 0.534950 & 0.664547 & -1.108995 \\ \mathrm{H} & -2.855534 & 1.151929 & -0.911947 \\ \mathrm{H} & -1.946093 & 0.783005 & -2.127113 \\ \mathrm{H} & -0.902725 & 2.408056 & 1.037859 \\ \mathrm{C} & 1.388657 & 0.179384 & -0.268938 \\ \mathrm{C} & 2.885407 & 0.320170 & -0.473318 \\ \mathrm{O} & 0.860371 & -0.415483 & 0.747277 \\ \mathrm{~N} & 3.759746 & -0.219682 & 0.541556 \\ \mathrm{H} & 3.071194 & 1.392869 & -0.606520 \\ \mathrm{H} & 3.108437 & -0.126002 & -1.450586 \\ \mathrm{H} & 3.654204 & -1.222388 & 0.656559 \\ \mathrm{H} & 3.624550 & 0.223945 & 1.444488 \\ \mathrm{O} & -0.958220 & -1.766397 & -0.741867 \\ \mathrm{H} & -0.171094 & -2.325933 & -0.832856 \\ \mathrm{H} & -1.610895 & -2.031521 & -1.409387\end{array}$

$\left[\left(\mathrm{NH}_{2} \mathrm{CH}_{2} \mathrm{COO}^{-}\right){ }^{1} \mathrm{Al}\left(\mathrm{H}_{2} \mathrm{O}\right)_{3}(\mathrm{OH})\right]^{+}(\mathrm{b})$ $\mathrm{E}=-831.5660106$
$\begin{array}{llll}\text { AL } & -0.959587 & 0.001497 & -0.113419\end{array}$
$\begin{array}{llll}\mathrm{O} & -1.892462 & 0.004602 & 1.663483\end{array}$
$\begin{array}{llll}\mathrm{O} & -1.059854 & 2.008766 & 0.013358\end{array}$
$\mathrm{H} \quad-1.862771 \quad 0.783707 \quad 2.241620$
$\mathrm{H} \quad-1.900626 \quad-0.791018 \quad 2.219435$
$\begin{array}{llll}\text { O } & -1.064362 & -2.006699 & 0.022843\end{array}$
$\begin{array}{llll}\mathrm{O} & -2.339051 & -0.003861 & -1.167184\end{array}$
O $\quad 0.523407 \quad-0.004860 \quad-1.348832$
$\mathrm{H} \quad-1.620568 \quad 2.299474 \quad-0.729951$
$\mathrm{H} \quad-0.301896 \quad 2.611487 \quad 0.085227$
$\mathrm{H} \quad-1.623591 \quad-2.294942 \quad-0.722720$
$\mathrm{H} \quad-0.308168 \quad-2.611462 \quad 0.096452$
$\mathrm{H} \quad-3.268953 \quad 0.005651 \quad-0.922299$
C $\quad 1.335354 \quad-0.001470 \quad-0.353373$
$\begin{array}{llll}\text { C } & 2.842221 & -0.004241 & -0.536852\end{array}$
$\begin{array}{llll}\mathrm{O} & 0.755930 & 0.003970 & 0.804840\end{array}$
$\begin{array}{llll}\mathrm{N} & 3.671061 & 0.000559 & 0.646888\end{array}$
$\mathrm{H} \quad 3.075563 \quad 0.859842 \quad-1.171010$
$\mathrm{H} \quad 3.073160 \quad-0.875391 \quad-1.162208$
$\begin{array}{llll}\mathrm{H} & 3.528532 & -0.819619 & 1.227109\end{array}$
$\mathrm{H} \quad 3.532209 \quad 0.827820 \quad 1.217865$

$\left[\left(\mathrm{NH}_{2} \mathrm{CH}_{2} \mathrm{COO}^{-}\right){ }^{1} \mathrm{Al}\left(\mathrm{H}_{2} \mathrm{O}\right)_{3}(\mathrm{OH})\right]^{+}(\mathrm{c})$ $\mathrm{E}=-831.5729251$
$\begin{array}{llll}\text { AL } & 0.916381 & 0.008588 & -0.157964\end{array}$
$\begin{array}{llll}\mathrm{O} & 2.339392 & 0.125568 & 1.219262\end{array}$
O $\quad 0.882902 \quad 2.016156 \quad-0.191580$ 


\begin{tabular}{|c|c|c|c|}
\hline $\mathrm{H}$ & 3.226795 & -0.027747 & \\
\hline $\mathrm{H}$ & & -0.326261 & \\
\hline $\mathrm{O}$ & 1.038952 & -2.017350 & \\
\hline $\mathrm{O}$ & -0.427244 & & \\
\hline $\mathrm{O}$ & 2.150270 & -0.059618 & \\
\hline $\mathrm{H}$ & 1.563352 & & \\
\hline $\mathrm{H}$ & 0.714528 & 2.579980 & \\
\hline $\mathrm{H}$ & 1.5316 & -2.3 & \\
\hline $\mathrm{H}$ & 0.3179 & -2.6 & \\
\hline $\mathrm{C}$ & -1.3360 & -0.0 & \\
\hline $\mathrm{H}$ & & -0.0 & \\
\hline $\mathrm{C}$ & -2.8159 & & \\
\hline $\mathrm{O}$ & -0.8718 & & \\
\hline $\mathrm{N}$ & -3.7 & -0.0 & \\
\hline $\mathrm{H}$ & -2.9 & -0.7 & \\
\hline $\mathrm{H}$ & -2.97 & 0.9 & \\
\hline $\mathrm{H}$ & -3.6 & 0.7 & \\
\hline $\mathrm{H}$ & -3.6 & -0.9 & \\
\hline \multirow{2}{*}{\multicolumn{4}{|c|}{$\begin{array}{l}{\left[\left(\mathrm{NH}_{2} \mathrm{CH}_{2} \mathrm{COO}^{-}\right)^{1} \mathrm{Al}\left(\mathrm{H}_{2} \mathrm{O}\right)_{3}(\mathrm{OH})\right]^{+}(\mathrm{d})} \\
\mathrm{E}=-831.5655978\end{array}$}} \\
\hline & & & \\
\hline & & & \\
\hline $\mathrm{O}$ & & -0.7 & \\
\hline $\mathrm{O}$ & & & \\
\hline $\mathrm{H}$ & & $-0 .($ & \\
\hline $\mathrm{H}$ & 1.9 & -1. & \\
\hline $\mathrm{O}$ & & & \\
\hline $\mathrm{O}$ & -0.5 & & \\
\hline $\mathrm{H}$ & & & -0 \\
\hline $\mathrm{H}$ & 1.9 & & -2 . \\
\hline $\mathrm{H}$ & 0.9005 & & \\
\hline $\mathrm{C}$ & -1.3 & & -0.2 \\
\hline $\mathrm{C}$ & -2.8 & 0.3 & \\
\hline $\mathrm{O}$ & -0.8 & -0. & \\
\hline $\mathrm{N}$ & -3.7 & -0.2 & \\
\hline $\mathrm{H}$ & $-3.0^{\prime}$ & & \\
\hline $\mathrm{H}$ & -3.1093 & -0.1 & \\
\hline $\mathrm{H}$ & -3.654912 & -1.2 & \\
\hline $\mathrm{H}$ & -3.623939 & & \\
\hline $\mathrm{O}$ & 0.959652 & -1.775361 & -0.7 \\
\hline $\mathrm{H}$ & 0.172964 & -2.33 & -0.8 \\
\hline $\mathrm{H}$ & 1.613598 & -2.049891 & -1.381982 \\
\hline \multicolumn{4}{|c|}{$\begin{array}{l}{\left[\left(\mathrm{NH}_{3}{ }^{+} \mathrm{CH}_{2} \mathrm{COO}^{-}\right){ }^{1} \mathrm{Sc}\left(\mathrm{H}_{2} \mathrm{O}\right)_{4}\right]^{3+}} \\
\mathrm{E}=-712.7527845\end{array}$} \\
\hline $\mathrm{C}$ & & 0.00314 & -0.604216 \\
\hline
\end{tabular}




\begin{tabular}{|c|c|c|c|}
\hline $\mathrm{H}$ & 3.274288 & 0.890386 & -1.197213 \\
\hline $\mathrm{H}$ & 3.277597 & -0.874476 & -1.209916 \\
\hline $\mathrm{C}$ & 1.537355 & -0.000873 & -0.317088 \\
\hline $\mathrm{O}$ & 0.706834 & 0.000864 & -1.283309 \\
\hline $\mathrm{O}$ & 1.035541 & -0.004599 & 0.869390 \\
\hline FE & -0.865146 & -0.001050 & 0.052012 \\
\hline $\mathrm{N}$ & 3.895211 & -0.003826 & 0.634733 \\
\hline $\mathrm{H}$ & 3.751035 & -0.838970 & 1.218510 \\
\hline $\mathrm{H}$ & 4.890571 & 0.002142 & 0.361032 \\
\hline $\mathrm{O}$ & -0.979365 & 2.071582 & 0.203573 \\
\hline $\mathrm{O}$ & -0.990198 & -2.072617 & 0.197213 \\
\hline $\mathrm{O}$ & -2.336196 & 0.002401 & 1.501367 \\
\hline $\mathrm{H}$ & -2.743772 & 0.790496 & 1.912468 \\
\hline $\mathrm{O}$ & -2.060995 & 0.005139 & -1.623877 \\
\hline $\mathrm{H}$ & -3.037754 & 0.009287 & -1.682315 \\
\hline $\mathrm{H}$ & -1.707083 & 0.004668 & -2.536832 \\
\hline $\mathrm{H}$ & -0.585819 & 2.637298 & 0.896183 \\
\hline $\mathrm{H}$ & -1.341184 & 2.663660 & -0.485560 \\
\hline $\mathrm{H}$ & -2.746063 & -0.783221 & 1.914914 \\
\hline $\mathrm{H}$ & -1.361105 & -2.660142 & -0.491036 \\
\hline $\mathrm{H}$ & -0.595133 & -2.643194 & 0.884990 \\
\hline $\mathrm{H}$ & 3.744695 & 0.820371 & 1.232299 \\
\hline \multicolumn{4}{|c|}{$\begin{array}{l}{\left[\left(\mathrm{NH}_{3}^{+} \mathrm{CH}_{2} \mathrm{COO}^{-}\right){ }^{6} \mathrm{Fe}\left(\mathrm{H}_{2} \mathrm{O}\right)_{3}\left(\mathrm{OH}^{-}\right)\right]^{2+}(\mathrm{a})} \\
\mathrm{E}=-712.6582755\end{array}$} \\
\hline $\mathrm{C}$ & 1.542104 & -0.364219 & -0.081505 \\
\hline $\mathrm{C}$ & 3.041844 & -0.631726 & -0.145633 \\
\hline $\mathrm{O}$ & 1.121070 & 0.710454 & 0.464161 \\
\hline $\mathrm{N}$ & 3.789464 & 0.646326 & 0.173458 \\
\hline $\mathrm{H}$ & 3.309427 & -1.386466 & 0.597444 \\
\hline $\mathrm{H}$ & 3.335376 & -0.991766 & -1.132157 \\
\hline $\mathrm{O}$ & 0.726643 & -1.204438 & -0.559295 \\
\hline $\mathrm{H}$ & 3.232414 & 1.203096 & 0.839909 \\
\hline $\mathrm{H}$ & 4.716651 & 0.460918 & 0.573256 \\
\hline $\mathrm{H}$ & 3.921644 & 1.226844 & -0.662969 \\
\hline $\mathrm{O}$ & -2.195685 & -1.514618 & -0.530257 \\
\hline $\mathrm{H}$ & -3.054304 & -1.378358 & -0.968711 \\
\hline $\mathrm{H}$ & -1.809377 & -2.324589 & -0.909070 \\
\hline $\mathrm{O}$ & -1.063363 & -0.710575 & 1.874144 \\
\hline $\mathrm{H}$ & -0.503363 & -0.546639 & 2.649855 \\
\hline $\mathrm{H}$ & -1.606541 & -1.494702 & 2.061131 \\
\hline $\mathrm{O}$ & -2.168816 & 1.536242 & 0.692450 \\
\hline $\mathrm{H}$ & -2.498170 & 1.632643 & 1.600959 \\
\hline $\mathrm{H}$ & -2.442301 & 2.308997 & 0.167733 \\
\hline FE & -0.878046 & 0.127018 & -0.153527 \\
\hline $\mathrm{O}$ & -1.021513 & 0.994106 & -1.693283 \\
\hline
\end{tabular}




\begin{tabular}{|c|c|c|c|}
\hline $\mathrm{H}$ & 0.988882 & & \\
\hline \multirow{2}{*}{\multicolumn{4}{|c|}{$\begin{array}{l}{\left[\left(\mathrm{NH}_{3}{ }^{+} \mathrm{CH}_{2} \mathrm{COO}^{-}\right){ }^{6} \mathrm{Fe}\left(\mathrm{H}_{2} \mathrm{O}\right)_{3}\left(\mathrm{OH}^{-}\right)\right]^{2+}(\mathrm{b})} \\
\mathrm{E}=-712.6609761\end{array}$}} \\
\hline & & & \\
\hline $\mathrm{C}$ & & 0.053578 & 373088 \\
\hline & & & \\
\hline & 1.122711 & -0.0 & \\
\hline $\mathrm{N}$ & & & \\
\hline $\mathrm{H}$ & & & \\
\hline 1 & 3.23 & -0.5 & \\
\hline & & & \\
\hline 1 & & & \\
\hline $\mathrm{H}$ & & & \\
\hline 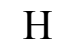 & & & \\
\hline & & & \\
\hline $\mathrm{H}$ & & & \\
\hline $\mathrm{H}$ & & & \\
\hline $\mathrm{O}$ & -0.8 & & \\
\hline $\mathrm{H}$ & -1.3 & & \\
\hline $\mathrm{H}$ & & & \\
\hline $\mathrm{O}$ & -0.8 & & \\
\hline $\mathrm{H}$ & & & \\
\hline $\mathrm{H}$ & -0.8 & & \\
\hline & & & \\
\hline $\mathrm{O}$ & & & \\
\hline $\mathrm{H}$ & & & \\
\hline \multicolumn{4}{|c|}{$\left[\left(\mathrm{NH}_{3}{ }^{+} \mathrm{CH}_{2} \mathrm{COO}^{-}\right){ }^{6} \mathrm{Fe}\left(\mathrm{H}_{2} \mathrm{O}\right)_{3}\left(\mathrm{OH}^{-}\right)\right]^{2+}(\mathrm{c})$} \\
\hline \multicolumn{4}{|c|}{$E=-712.6709565$} \\
\hline & -1.214230 & & \\
\hline & -2.2 & & \\
\hline & -0.5 & & \\
\hline & & & \\
\hline & -2.9 & -0. & \\
\hline & -1.9 & -1.9 & \\
\hline $\mathrm{O}$ & -2.19 & & \\
\hline & -0.86 & & \\
\hline $\mathrm{H}$ & 0.45 & & -0.0 \\
\hline & -2.54 & -2.3 & \\
\hline $\mathrm{H}$ & -1.44 & -2.6 & \\
\hline & -2.23 & 0.1 & \\
\hline $\mathrm{C}$ & & -0.0 & \\
\hline $\mathrm{C}$ & 2.995289 & -0.98 & -0.0 \\
\hline $\mathrm{O}$ & 0.629849 & -0.6 & \\
\hline $\mathrm{O}$ & 1.978987 & 1.171584 & -0.06 \\
\hline $\mathrm{N}$ & 4.239626 & -0.127862 & -0.05749 \\
\hline
\end{tabular}




$$
\begin{aligned}
& \text { H } \quad 3.004726 \quad-1.599209 \quad 0.896243 \\
& \text { H } \quad 2.972182 \quad-1.649978 \quad-0.875434 \\
& \mathrm{H} \quad 4.818157 \quad-0.316225 \quad-0.882648 \\
& \begin{array}{llll}
\mathrm{H} & 3.908050 & 0.856999 & -0.097204
\end{array} \\
& \mathrm{H} \quad 4.831723 \quad-0.242741 \quad 0.771571 \\
& {\left[\left(\mathrm{NH}_{3}{ }^{+} \mathrm{CH}_{2} \mathrm{COO}^{-}\right){ }^{6} \mathrm{Fe}\left(\mathrm{H}_{2} \mathrm{O}\right)_{3}\left(\mathrm{OH}^{-}\right)\right]^{2+}(\mathrm{d})} \\
& \mathrm{E}=-712.6591757 \\
& \begin{array}{llll}
\text { C } & -1.542097 & -0.364228 & -0.081492
\end{array} \\
& \begin{array}{llll}
\text { C } & -3.041836 & -0.631750 & -0.145575
\end{array} \\
& \begin{array}{llll}
\mathrm{O} & -1.121049 & 0.710509 & 0.464033
\end{array} \\
& \begin{array}{llll}
\mathrm{N} & -3.789471 & 0.646304 & 0.173466
\end{array} \\
& \mathrm{H} \quad-3.335378 \quad-0.991835 \quad-1.132080 \\
& \begin{array}{llll}
\mathrm{H} & -3.309407 & -1.386462 & 0.597534
\end{array} \\
& \begin{array}{llll}
\text { O } & -0.726635 & -1.204516 & -0.559162
\end{array} \\
& \mathrm{H} \quad-3.921768 \quad 1.226725 \quad-0.663010 \\
& \mathrm{H} \quad-4.716605 \quad 0.460907 \quad 0.573391 \\
& \mathrm{H} \quad-3.232365 \quad 1.203165 \quad 0.839795 \\
& \begin{array}{llll}
\text { O } & 2.195641 & -1.514690 & -0.530156
\end{array} \\
& \mathrm{H} \quad 3.054182 \quad-1.378486 \quad-0.968781 \\
& \text { H } \quad 1.809277 \quad-2.324717 \quad-0.908791 \\
& \begin{array}{llll}
\mathrm{O} & 1.063580 & -0.710548 & 1.874140
\end{array} \\
& \text { H } \quad 0.503720 \quad-0.546696 \quad 2.649970 \\
& \mathrm{H} \quad 1.606930 \quad-1.494582 \quad 2.061007 \\
& \begin{array}{llll}
\mathrm{O} & 2.168581 & 1.536500 & 0.692347
\end{array} \\
& \text { H } 2.442034 \quad 2.309172 \quad 0.167489 \\
& \begin{array}{llll}
\mathrm{H} & 2.498032 & 1.633026 & 1.600807
\end{array} \\
& \text { FE } \quad 0.878030 \quad 0.127027 \quad-0.153514 \\
& \begin{array}{llll}
\mathrm{O} & 1.021556 & 0.993942 & -1.693366
\end{array} \\
& \text { H } \quad 0.989062 \quad 0.949233 \quad-2.660512 \\
& \mathrm{E}=-712.3999163 \\
& {\left[\left(\mathrm{NH}_{2} \mathrm{CH}_{2} \mathrm{COO}^{-}\right)^{6} \mathrm{Fe}\left(\mathrm{H}_{2} \mathrm{O}\right)_{3}(\mathrm{OH})\right]^{+}(\mathrm{b})} \\
& \mathrm{E}=-712.3998649 \\
& {\left[\left(\mathrm{NH}_{2} \mathrm{CH}_{2} \mathrm{COO}^{-}\right)^{6} \mathrm{Fe}\left(\mathrm{H}_{2} \mathrm{O}\right)_{3}(\mathrm{OH})\right]^{+} \text {(c) }} \\
& \mathrm{E}=-712.3992962 \\
& {\left[\left(\mathrm{NH}_{2} \mathrm{CH}_{2} \mathrm{COO}^{-}\right)^{6} \mathrm{Fe}\left(\mathrm{H}_{2} \mathrm{O}\right)_{3}(\mathrm{OH})\right]^{+}(\mathrm{d})} \\
& \mathrm{E}=-712.3999163 \\
& {\left[\left(\mathrm{NH}_{3}{ }^{+} \mathrm{CH}_{2} \mathrm{COO}^{-}\right){ }^{1} \mathrm{Sc}\left(\mathrm{H}_{2} \mathrm{O}\right)_{4}\right]^{3+}} \\
& \mathrm{E}=-1350.1738195 \\
& \begin{array}{llll}
\text { C } & 3.044370 & -0.000283 & 0.584228
\end{array}
\end{aligned}
$$




\begin{tabular}{|c|c|c|c|}
\hline $\mathrm{H}$ & 3.297489 & -0.882925 & 1.179710 \\
\hline $\mathrm{H}$ & 3.297442 & 0.881738 & 1.180669 \\
\hline $\mathrm{C}$ & 1.548474 & -0.000117 & 0.305548 \\
\hline $\mathrm{O}$ & 0.732546 & -0.001153 & 1.285366 \\
\hline $\mathrm{O}$ & 1.058460 & 0.001096 & -0.886057 \\
\hline $\mathrm{SC}$ & -0.921416 & 0.000006 & -0.058817 \\
\hline $\mathrm{N}$ & 3.892655 & 0.000467 & -0.666116 \\
\hline $\mathrm{H}$ & 3.731502 & 0.829296 & -1.253691 \\
\hline $\mathrm{H}$ & 4.892092 & 0.000530 & -0.410193 \\
\hline $\mathrm{O}$ & -1.074168 & -2.179150 & -0.214029 \\
\hline $\mathrm{O}$ & -1.074810 & 2.179276 & -0.211565 \\
\hline $\mathrm{O}$ & -2.501646 & 0.000771 & -1.575465 \\
\hline $\mathrm{H}$ & -2.935057 & -0.777007 & -1.980688 \\
\hline $\mathrm{O}$ & -2.075200 & -0.001010 & 1.776048 \\
\hline $\mathrm{H}$ & -3.043388 & -0.001584 & 1.921317 \\
\hline $\mathrm{H}$ & -1.662852 & -0.001140 & 2.664817 \\
\hline $\mathrm{H}$ & -0.739271 & -2.760602 & -0.925081 \\
\hline $\mathrm{H}$ & -1.436303 & -2.776642 & 0.471006 \\
\hline $\mathrm{H}$ & -2.933956 & 0.779046 & -1.980917 \\
\hline $\mathrm{H}$ & -1.439424 & 2.775735 & 0.473053 \\
\hline $\mathrm{H}$ & -0.737480 & 2.761783 & -0.920599 \\
\hline $\mathrm{H}$ & 3.731822 & -0.827858 & -1.254484 \\
\hline \multicolumn{4}{|c|}{$\begin{array}{l}{\left[\left(\mathrm{NH}_{3}{ }^{+} \mathrm{CH}_{2} \mathrm{COO}^{-}\right){ }^{1} \mathrm{Sc}\left(\mathrm{H}_{2} \mathrm{O}\right)_{3}\left(\mathrm{OH}^{-}\right)\right]^{2+}(\mathrm{a})} \\
\mathrm{E}=-1350.070825\end{array}$} \\
\hline $\mathrm{SC}$ & 0.938274 & 0.129765 & 0.180320 \\
\hline $\mathrm{O}$ & 2.234939 & -1.632776 & 0.592517 \\
\hline $\mathrm{O}$ & 1.197657 & -0.670866 & -1.973255 \\
\hline $\mathrm{H}$ & 1.858925 & -2.526228 & 0.685088 \\
\hline $\mathrm{H}$ & 2.990302 & -1.588448 & 1.205429 \\
\hline $\mathrm{O}$ & 2.284753 & 1.665638 & -0.665705 \\
\hline $\mathrm{O}$ & -1.134812 & 0.727709 & -0.439794 \\
\hline $\mathrm{O}$ & 1.096965 & 0.940129 & 1.817270 \\
\hline $\mathrm{O}$ & -0.763419 & -1.256877 & 0.476536 \\
\hline $\mathrm{H}$ & 1.824450 & -1.367917 & -2.230996 \\
\hline $\mathrm{H}$ & 0.671725 & -0.467792 & -2.764100 \\
\hline $\mathrm{H}$ & 2.664319 & 1.790966 & -1.551103 \\
\hline $\mathrm{H}$ & 2.591008 & 2.400024 & -0.105333 \\
\hline $\mathrm{C}$ & -1.561145 & -0.375865 & 0.053462 \\
\hline $\mathrm{H}$ & 1.173154 & 1.289518 & 2.714378 \\
\hline $\mathrm{C}$ & -3.067891 & -0.616702 & 0.121232 \\
\hline $\mathrm{N}$ & -3.785514 & 0.689330 & -0.150945 \\
\hline $\mathrm{H}$ & -3.358353 & -1.004335 & 1.097876 \\
\hline $\mathrm{H}$ & -3.353816 & -1.342178 & -0.643437 \\
\hline $\mathrm{H}$ & -3.152386 & 1.293022 & -0.701742 \\
\hline $\mathrm{H}$ & -4.664714 & 0.553601 & -0.661974 \\
\hline
\end{tabular}




\begin{tabular}{|c|c|c|c|}
\hline $\mathrm{H}$ & 4.004226 & & \\
\hline \multirow{2}{*}{\multicolumn{4}{|c|}{$\begin{array}{l}{\left[\left(\mathrm{NH}_{3}{ }^{+} \mathrm{CH}_{2} \mathrm{COO}^{-}\right){ }^{1} \mathrm{Sc}\left(\mathrm{H}_{2} \mathrm{O}\right)_{3}\left(\mathrm{OH}^{-}\right)\right]^{2+}(\mathrm{b})} \\
\mathrm{E}=-135007039\end{array}$}} \\
\hline \multicolumn{3}{|c|}{$\mathrm{E}=-1350.07039$} & \\
\hline & 1.061458 & 0.000201 & 5363 \\
\hline & 1.027470 & & \\
\hline & 1.029257 & & \\
\hline $\mathrm{H}$ & & & \\
\hline $\mathrm{H}$ & 0.69 & -2.8 & \\
\hline $\mathrm{O}$ & 1.96 & -0.0 & \\
\hline $\mathrm{O}$ & -0.72 & & \\
\hline $\mathrm{O}$ & -1.1 & -0.0 & \\
\hline $\mathrm{O}$ & & & \\
\hline $\mathrm{H}$ & 1.5 & & \\
\hline & & & \\
\hline $\mathrm{H}$ & & & \\
\hline $\mathrm{H}$ & 2.9 & & \\
\hline $\mathrm{C}$ & -1.5 & & \\
\hline $\mathrm{H}$ & 3.2 & & \\
\hline $\mathrm{C}$ & & & \\
\hline $\mathrm{N}$ & -3.7 & & \\
\hline $\mathrm{H}$ & & & \\
\hline $\mathrm{H}$ & & & \\
\hline $\mathrm{H}$ & & & \\
\hline $\mathrm{H}$ & & & \\
\hline $\mathrm{H}$ & & & \\
\hline \multicolumn{4}{|c|}{$\left[\left(\mathrm{NH}_{3}{ }^{+} \mathrm{CH}_{2} \mathrm{COO}^{-}\right){ }^{1} \mathrm{Sc}\left(\mathrm{H}_{2} \mathrm{O}\right)_{3}\left(\mathrm{OH}^{-}\right)\right]^{2+}(\mathrm{c})$} \\
\hline \multicolumn{4}{|c|}{$\mathrm{E}=-1350.073197$} \\
\hline & -1.016116 & & \\
\hline & -0.9 & & \\
\hline & & -0.0 & \\
\hline & & & \\
\hline & -0.8 & & \\
\hline $\mathrm{O}$ & -2.1 & & \\
\hline & 1.5 & & \\
\hline$H$ & -2.6 & & \\
\hline $\mathrm{C}$ & 3.00 & -0.0 & \\
\hline $\mathrm{O}$ & 0.66 & & \\
\hline $\mathrm{N}$ & 3.75 & -0.0 & \\
\hline & 3.266 & -0.8 & \\
\hline $\mathrm{H}$ & & & \\
\hline $\mathrm{H}$ & 3.050079 & -0.002305 & \\
\hline $\mathrm{H}$ & 4.35 & -0.8 & -0.7 \\
\hline $\mathrm{H}$ & 4.354179 & 0.828229 & -0.722739 \\
\hline $\mathrm{O}$ & -0.991570 & -2.221000 & -0.104174 \\
\hline
\end{tabular}




\begin{tabular}{cccc}
$\mathrm{H}$ & -0.812286 & -2.827271 & 0.633120 \\
$\mathrm{H}$ & -1.415912 & -2.738868 & -0.809992 \\
$\mathrm{O}$ & -2.409458 & -0.000019 & 1.522157 \\
$\mathrm{H}$ & -2.184566 & 0.000929 & 2.468119 \\
$\mathrm{H}$ & -3.380088 & -0.000186 & 1.454636 \\
\multicolumn{4}{|c}{} \\
{$\left[\mathrm{NH}_{3}{ }^{+} \mathrm{CH}_{2} \mathrm{COO}^{-}\right)$} & $\left.{ }^{1} \mathrm{Sc}\left(\mathrm{H}_{2} \mathrm{O}\right)_{3}\left(\mathrm{OH}^{-}\right)\right]^{2+}(\mathrm{d})$ \\
$\mathrm{E}=-1350.070825$ & & \\
$\mathrm{SC}$ & -0.938203 & -0.129920 & -0.180269 \\
$\mathrm{O}$ & -2.285874 & -1.664542 & 0.666281 \\
$\mathrm{O}$ & -2.234003 & 1.633083 & -0.593317 \\
$\mathrm{H}$ & -2.592172 & -2.399079 & 0.106128 \\
$\mathrm{H}$ & -2.666145 & -1.789119 & 1.551482 \\
$\mathrm{O}$ & 0.763444 & 1.256395 & -0.477623 \\
$\mathrm{O}$ & -1.096850 & -0.941608 & -1.816577 \\
$\mathrm{H}$ & -1.857287 & 2.526036 & -0.687877 \\
$\mathrm{H}$ & -2.990534 & 1.588679 & -1.204786 \\
$\mathrm{C}$ & 1.561112 & 0.375712 & -0.053757 \\
$\mathrm{H}$ & -1.173077 & -1.291718 & -2.713399 \\
$\mathrm{C}$ & 3.067870 & 0.616441 & -0.121537 \\
$\mathrm{O}$ & 1.134691 & -0.727461 & 0.440348 \\
$\mathrm{~N}$ & 3.785402 & -0.689446 & 0.151569 \\
$\mathrm{H}$ & 3.358458 & 1.003437 & -1.098394 \\
$\mathrm{H}$ & 3.353741 & 1.342363 & 0.642731 \\
$\mathrm{H}$ & 4.664792 & -0.553365 & 0.662179 \\
$\mathrm{H}$ & 3.152397 & -1.292575 & 0.703120 \\
$\mathrm{H}$ & 4.003752 & -1.192058 & -0.716091 \\
$\mathrm{O}$ & -1.197391 & 0.671741 & 1.973013 \\
$\mathrm{H}$ & -0.672536 & 0.467885 & 2.764373 \\
$\mathrm{H}$ & -1.822966 & 1.370175 & 2.229969 \\
& & &
\end{tabular}

\begin{tabular}{|c|c|c|c|}
\hline \multicolumn{4}{|c|}{$\begin{array}{l}{\left[\left(\mathrm{NH}_{2} \mathrm{CH}_{2} \mathrm{COO}^{-}\right){ }^{1} \mathrm{Sc}\left(\mathrm{H}_{2} \mathrm{O}\right)_{3}(\mathrm{OH})\right]^{+}(\mathrm{a})} \\
\mathrm{E}=-1349.804519\end{array}$} \\
\hline $\mathrm{SC}$ & 0.885686 & -0.044054 & -0.227569 \\
\hline $\mathrm{O}$ & 2.257851 & -1.780717 & 0.126214 \\
\hline $\mathrm{O}$ & 1.419767 & -0.139368 & 7072 \\
\hline $\mathrm{H}$ & 2.634129 & -2.217063 & 5560 \\
\hline $\mathrm{H}$ & 2.432549 & -2.3 & 032 \\
\hline $\mathrm{O}$ & -0.770739 & 1.298278 & 5207 \\
\hline $\mathrm{H}$ & 1.457219 & -0.09 & 357 \\
\hline $\mathrm{C}$ & -1.574699 & 0.291 & 0438 \\
\hline $\mathrm{C}$ & -3.081288 & 0.500 & 5443 \\
\hline $\mathrm{O}$ & -1.047790 & -0.886018 & 0.001550 \\
\hline $\mathrm{N}$ & -3.924917 & -0.674555 & -0.054088 \\
\hline $\mathrm{H}$ & -3.299190 & 1.132575 & -0.914396 \\
\hline $\mathrm{H}$ & -3.309204 & 1.128494 & 0.825078 \\
\hline
\end{tabular}




\begin{tabular}{|c|c|c|c|}
\hline $\mathrm{H}$ & & & \\
\hline $\mathrm{H}$ & & -1.237974 & \\
\hline $\mathrm{O}$ & 2.008612 & 1.852370 & \\
\hline $\mathrm{H}$ & .7481 & & \\
\hline $\mathrm{H}$ & 1.4088 & & \\
\hline & & & \\
\hline & & & \\
\hline & & & \\
\hline \multirow{2}{*}{\multicolumn{4}{|c|}{$\begin{array}{l}{\left[\left(\mathrm{NH}_{2} \mathrm{CH}_{2} \mathrm{COO}^{-}\right){ }^{1} \mathrm{Sc}\left(\mathrm{H}_{2} \mathrm{O}\right)_{3}(\mathrm{OH})\right]^{+}(\mathrm{b})} \\
\mathrm{E}=-1349.817016\end{array}$}} \\
\hline & & & \\
\hline & -0.909471 & -0.129042 & -0.190640 \\
\hline & & & \\
\hline & & & \\
\hline & & & \\
\hline & & & -0. \\
\hline & & & \\
\hline $\mathrm{O}$ & & & \\
\hline 1 & 7 & -0.7 & \\
\hline$\Gamma$ & & & \\
\hline $\mathrm{H}$ & & & \\
\hline $\mathrm{H}$ & & & \\
\hline $\mathrm{H}$ & & -1. & \\
\hline ) & & & \\
\hline $\mathrm{H}$ & & & \\
\hline $\mathrm{H}$ & -2.8 & & \\
\hline $\mathrm{O}$ & & & \\
\hline $\mathrm{H}$ & & -1. & \\
\hline $\mathrm{H}$ & & & \\
\hline $\mathrm{O}$ & & -0 . & \\
\hline $\mathrm{H}$ & & & \\
\hline $\mathrm{H}$ & & & \\
\hline \multirow{2}{*}{\multicolumn{4}{|c|}{$\begin{array}{l}{\left[\left(\mathrm{NH}_{2} \mathrm{CH}_{2} \mathrm{COO}^{-}\right){ }^{1} \mathrm{Sc}\left(\mathrm{H}_{2} \mathrm{O}\right)_{3}(\mathrm{OH})\right]^{+}(\mathrm{c})} \\
\mathrm{E}=-1349806561\end{array}$}} \\
\hline $\mathrm{E}=-1349.806561$ & & & \\
\hline & -0.953059 & -0.0 & \\
\hline & -2.13 & & \\
\hline $\mathrm{C}$ & $-0.961<$ & 2.236 & -0.1 \\
\hline & -3.09 & -0.000441 & \\
\hline I & -1.6979 & 0.001168 & 2.5 \\
\hline & -0.9611 & -2.236913 & -0.1 \\
\hline C & & -0.001733 & -1.5 \\
\hline & -1.422171 & 2.737914 & -0.8 \\
\hline $\mathrm{H}$ & -0.3865 & 2.848124 & 0.33 \\
\hline $\mathrm{H}$ & -1.421302 & -2.738440 & -0.840864 \\
\hline $\mathrm{H}$ & -0.384271 & -2.847470 & 0.334389 \\
\hline
\end{tabular}




$\begin{array}{crrc}\mathrm{H} & -2.951480 & -0.002330 & -2.214750 \\ \mathrm{C} & 1.513540 & 0.000622 & 0.296780 \\ \mathrm{C} & 3.010883 & 0.001331 & 0.572148 \\ \mathrm{O} & 1.049017 & -0.001091 & -0.905484 \\ \mathrm{O} & 0.665906 & 0.001847 & 1.268666 \\ \mathrm{~N} & 3.905892 & -0.001315 & -0.565250 \\ \mathrm{H} & 3.208376 & 0.871804 & 1.209536 \\ \mathrm{H} & 3.208548 & -0.865930 & 1.213823 \\ \mathrm{H} & 3.774803 & -0.822647 & -1.147956 \\ \mathrm{H} & 3.774488 & 0.817056 & -1.152046\end{array}$

$\left[\left(\mathrm{NH}_{2} \mathrm{CH}_{2} \mathrm{COO}^{-}\right){ }^{1} \mathrm{Sc}\left(\mathrm{H}_{2} \mathrm{O}\right)_{3}(\mathrm{OH})\right]^{+}(\mathrm{d})$

$\mathrm{E}=-1349.803522$

$\begin{array}{lccc}\mathrm{SC} & -0.870539 & -0.092446 & -0.212952 \\ \mathrm{O} & -2.218785 & -1.752316 & 0.455463 \\ \mathrm{O} & -1.321070 & -0.596160 & -1.947156 \\ \mathrm{H} & -2.529426 & -2.361899 & -0.233989 \\ \mathrm{H} & -2.394404 & -2.150093 & 1.321549 \\ \mathrm{O} & 0.782679 & 1.264952 & -0.304930 \\ \mathrm{H} & -1.261305 & -0.787774 & -2.889418 \\ \mathrm{C} & 1.577262 & 0.299449 & 0.003796 \\ \mathrm{C} & 3.072142 & 0.497557 & 0.022852 \\ \mathrm{O} & 1.042682 & -0.829894 & 0.323949 \\ \mathrm{~N} & 3.784994 & -0.757561 & -0.172880 \\ \mathrm{H} & 3.313843 & 1.200758 & -0.778840 \\ \mathrm{H} & 3.280850 & 1.029093 & 0.971522 \\ \mathrm{H} & 3.581213 & -1.422670 & 0.567620 \\ \mathrm{H} & 4.787692 & -0.597218 & -0.191240 \\ \mathrm{O} & -2.042702 & 1.836571 & -0.256395 \\ \mathrm{H} & -2.717804 & 1.958469 & -0.944610 \\ \mathrm{H} & -1.472407 & 2.624751 & -0.281813 \\ \mathrm{O} & -1.080564 & 0.312340 & 2.077332 \\ \mathrm{H} & -0.357586 & 0.104758 & 2.690313 \\ \mathrm{H} & -1.638653 & 0.980136 & 2.505047\end{array}$

$\left[\left(\mathrm{NH}_{3}{ }^{+} \mathrm{CH}_{2} \mathrm{COO}^{-}\right){ }^{1} \mathrm{Y}\left(\mathrm{H}_{2} \mathrm{O}\right)_{6}\right]^{3+}$

$\mathrm{E}=-780.8350903$

$\begin{array}{cccc}\mathrm{Y} & -0.704048 & 0.037822 & -0.029380 \\ \mathrm{O} & -3.035378 & 0.457601 & 0.503692 \\ \mathrm{O} & -0.358682 & 0.926564 & -2.252780 \\ \mathrm{H} & -3.805378 & 0.217718 & -0.042677 \\ \mathrm{H} & -3.401064 & 0.826339 & 1.327595 \\ \mathrm{O} & -0.598048 & 0.367875 & 2.351364 \\ \mathrm{O} & -1.942511 & -1.286986 & -1.650110 \\ \mathrm{O} & -1.209115 & -2.120476 & 1.003071 \\ \mathrm{O} & -0.767853 & 2.451365 & 0.193062\end{array}$




\begin{tabular}{|c|c|c|c|}
\hline $\mathrm{O}$ & & & \\
\hline $\mathrm{O}$ & & & \\
\hline $\mathrm{H}$ & & & \\
\hline $\mathrm{H}$ & & & \\
\hline $\mathrm{H}$ & & -0.329276 & \\
\hline $\mathrm{H}$ & & & \\
\hline $\mathrm{H}$ & & -2.24 & \\
\hline $\mathrm{H}$ & & & \\
\hline $\mathrm{H}$ & & -2.8 & \\
\hline $\mathrm{H}$ & 56 & -2.4 & \\
\hline $\mathrm{H}$ & & & \\
\hline $\mathrm{H}$ & -1.5 & 3.0 & \\
\hline $\mathrm{C}$ & & & \\
\hline $\mathrm{C}$ & & -0.7 & \\
\hline $\mathrm{N}$ & & & \\
\hline $\mathrm{H}$ & & & \\
\hline $\mathrm{H}$ & & & \\
\hline $\mathrm{H}$ & & & \\
\hline $\mathrm{H}$ & & & \\
\hline $\mathrm{H}$ & & & \\
\hline \multicolumn{4}{|c|}{$\begin{array}{l}{\left[\left(\mathrm{NH}_{3}{ }^{+} \mathrm{CH}_{2} \mathrm{COO}^{-}\right){ }^{1} \mathrm{Y}\left(\mathrm{H}_{2} \mathrm{O}\right)_{5}\left(\mathrm{OH}^{-}\right)\right]^{2+} \text { (a) }} \\
\mathrm{E}=-780.6780785\end{array}$} \\
\hline $\mathrm{Y}$ & & & \\
\hline $\mathrm{O}$ & & & \\
\hline $\mathrm{O}$ & & & \\
\hline $\mathrm{H}$ & & & \\
\hline C & & -0.4 & \\
\hline $\mathrm{C}$ & & -0.7 & \\
\hline $\mathrm{C}$ & & & \\
\hline $\mathrm{N}$ & & & \\
\hline $\mathrm{H}$ & & -1.48 & \\
\hline $\mathrm{H}$ & 26 & -1.2 & \\
\hline $\mathrm{H}$ & & & \\
\hline $\mathrm{H}$ & & & \\
\hline $\mathrm{H}$ & & & \\
\hline $\mathrm{O}$ & & & \\
\hline $\mathrm{H}$ & -3.7169 & & \\
\hline $\mathrm{H}$ & & -0.5 & \\
\hline $\mathrm{O}$ & -1.652377 & -1.83 & \\
\hline $\mathrm{H}$ & & -2.79 & \\
\hline $\mathrm{H}$ & & & \\
\hline $\mathrm{O}$ & -1.224913 & 2.470314 & -0.2 \\
\hline $\mathrm{H}$ & & 3.226920 & \\
\hline $\mathrm{H}$ & -1.181771 & 2.796444 & -1.129699 \\
\hline $\mathrm{O}$ & -0.495874 & 0.991112 & 2.178108 \\
\hline
\end{tabular}




\begin{tabular}{|c|c|c|c|}
\hline $\mathrm{H}$ & & & \\
\hline $\mathrm{H}$ & & & \\
\hline $\mathrm{O}$ & & & \\
\hline $\mathrm{H}$ & & & \\
\hline $\mathrm{H}$ & & & \\
\hline \multicolumn{4}{|c|}{$\begin{array}{l}{\left[\left(\mathrm{NH}_{3}{ }^{+} \mathrm{CH}_{2} \mathrm{COO}^{-}\right){ }^{1} \mathrm{Y}\left(\mathrm{H}_{2} \mathrm{O}\right)_{5}\left(\mathrm{OH}^{-}\right)\right]^{2+}(\mathrm{a})} \\
\mathrm{E}=-780.6866825\end{array}$} \\
\hline & -1.033069 & 0.074988 & -0.117007 \\
\hline & & & \\
\hline & 2.5 & -1.0 & \\
\hline 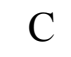 & 3.5 & & \\
\hline & & & \\
\hline $\mathbf{N}$ & & & \\
\hline $\mathrm{H}$ & & & \\
\hline H & 3.6 & & \\
\hline $\mathrm{H}$ & & & \\
\hline $\mathrm{H}$ & & & \\
\hline $\mathrm{H}$ & & & \\
\hline $\mathrm{O}$ & & & \\
\hline $\mathrm{H}$ & & -2. & \\
\hline $\mathrm{H}$ & & & \\
\hline $\mathrm{O}$ & & -1.7 & \\
\hline $\mathrm{H}$ & & -2. & \\
\hline $\mathrm{H}$ & & -1.6 & \\
\hline $\mathrm{O}$ & & & \\
\hline $\mathrm{H}$ & -1.7 & 2.8 & \\
\hline $\mathrm{H}$ & -0.6 & & \\
\hline $\mathrm{O}$ & & & \\
\hline $\mathrm{H}$ & & & \\
\hline $\mathrm{H}$ & & & \\
\hline $\mathrm{O}$ & & -1.1 & \\
\hline $\mathrm{H}$ & & & \\
\hline $\mathrm{H}$ & & $-1 .($ & \\
\hline $\mathrm{O}$ & -2.2 & 0.6 & -1.6 \\
\hline $\mathrm{H}$ & -2.78 & 0.908232 & -2.41 \\
\hline \multicolumn{4}{|c|}{$\left[\left(\mathrm{NH}_{3}{ }^{+} \mathrm{CH}_{2} \mathrm{COO}^{-}\right)^{1} \mathrm{Y}\left(\mathrm{H}_{2} \mathrm{O}\right)_{5}\left(\mathrm{OH}^{-}\right)\right]^{2+}(\mathrm{b})$} \\
\hline Y & -0.737529 & -0.020491 & \\
\hline $\mathrm{C}$ & & 0.27 & -0.2 \\
\hline $\mathrm{O}$ & & -0.757095 & 0.34 \\
\hline & & & -0.4 \\
\hline $\mathrm{O}$ & 1.282624 & 1.147742 & -0.761852 \\
\hline $\mathrm{N}$ & & -0.689804 & \\
\hline $\mathrm{H}$ & 3.848494 & 0.388441 & -1.477877 \\
\hline
\end{tabular}




\begin{tabular}{|c|c|c|c|}
\hline $\mathrm{H}$ & & & \\
\hline $\mathrm{H}$ & & & \\
\hline $\mathrm{H}$ & & & \\
\hline $\mathrm{H}$ & & & \\
\hline $\mathrm{O}$ & .745291 & -0.674096 & \\
\hline $\mathrm{H}$ & & & \\
\hline $\mathrm{H}$ & & & \\
\hline $\mathrm{O}$ & & & \\
\hline $\mathrm{H}$ & & -2.7 & \\
\hline $\mathrm{H}$ & & -3.1 & \\
\hline $\mathrm{O}$ & & & \\
\hline $\mathrm{H}$ & 61 & 2.5 & 1.7 \\
\hline $\mathrm{H}$ & & & \\
\hline $\mathrm{O}$ & -3.1 & -0.0 & \\
\hline $\mathrm{H}$ & & & \\
\hline $\mathrm{H}$ & -3.7 & -0 . & \\
\hline $\mathrm{O}$ & & & \\
\hline $\mathrm{H}$ & -0.6 & & \\
\hline $\mathrm{H}$ & & & \\
\hline $\mathrm{O}$ & -1.6 & -0.6 & \\
\hline $\mathrm{H}$ & -1.86 & & \\
\hline \multicolumn{4}{|c|}{$\begin{array}{l}{\left[\left(\mathrm{NH}_{3}{ }^{+} \mathrm{CH}_{2} \mathrm{COO}^{-}\right){ }^{1} \mathrm{Y}\left(\mathrm{H}_{2} \mathrm{O}\right)_{5}\left(\mathrm{OH}^{-}\right)\right]^{2+}(\mathrm{c})} \\
\mathrm{E}=-780.6792913\end{array}$} \\
\hline & & & \\
\hline & & & \\
\hline $\mathrm{C}$ & & -0.8 & \\
\hline C & 3.5 & & -0. \\
\hline $\mathrm{O}$ & & & \\
\hline I & & -0.6 & \\
\hline F & & & -0 \\
\hline $\mathrm{H}$ & & & \\
\hline $\mathrm{H}$ & 3.4 & -1.3 & -0.1 \\
\hline $\mathrm{H}$ & 4.75 & -0.8 & \\
\hline $\mathrm{H}$ & & -0.8 & \\
\hline $\mathrm{O}$ & -0.8 & & \\
\hline $\mathrm{H}$ & & & -2.6 \\
\hline $\mathrm{H}$ & -0.2 & 2.1 & -2.1 \\
\hline $\mathrm{O}$ & & & \\
\hline $\mathrm{H}$ & -1.860 & -1.8 & -2.09 \\
\hline $\mathrm{H}$ & -0.38 & -1.8 & -2.60 \\
\hline $\mathrm{O}$ & -0.309927 & 0.062971 & $2.36 \mathrm{~S}$ \\
\hline $\mathrm{H}$ & -0.526249 & 0.82 & \\
\hline $\mathrm{H}$ & -0.417926 & -0.740028 & 2.904033 \\
\hline $\mathrm{O}$ & -0.993335 & -2.252701 & 1.087763 \\
\hline $\mathrm{H}$ & -1.931233 & -2.507828 & 1.122131 \\
\hline
\end{tabular}




$\begin{array}{lrrc}\mathrm{H} & -0.494454 & -3.053305 & 0.861783 \\ \mathrm{O} & -1.426223 & 2.207530 & 0.891612 \\ \mathrm{H} & -0.907933 & 3.027033 & 0.862523 \\ \mathrm{H} & -2.364069 & 2.456126 & 0.927426 \\ \mathrm{O} & -2.850069 & -0.267821 & -0.044138 \\ \mathrm{H} & -3.812895 & -0.251643 & -0.040436\end{array}$

$\left[\left(\mathrm{NH}_{3}{ }^{+} \mathrm{CH}_{2} \mathrm{COO}^{-}\right){ }^{1} \mathrm{Y}\left(\mathrm{H}_{2} \mathrm{O}\right)_{5}\left(\mathrm{OH}^{-}\right)\right]^{2+}(\mathrm{d})$ $\mathrm{E}=-780.6737364$

$\begin{array}{cccc}\mathrm{Y} & -0.710263 & -0.004280 & 0.091706 \\ \mathrm{C} & 2.093296 & 0.002674 & -0.330346 \\ \mathrm{O} & 1.665097 & 0.129712 & 0.859915 \\ \mathrm{C} & 3.616153 & -0.051425 & -0.525850 \\ \mathrm{O} & 1.334335 & -0.084089 & -1.334422 \\ \mathrm{~N} & 4.252528 & 0.104103 & 0.841575 \\ \mathrm{H} & 3.910532 & -1.006736 & -0.960928 \\ \mathrm{H} & 3.952752 & 0.749451 & -1.184267 \\ \mathrm{H} & 3.447122 & 0.171263 & 1.502053 \\ \mathrm{H} & 4.823030 & 0.950982 & 0.920333 \\ \mathrm{H} & 4.839324 & -0.694194 & 1.100643 \\ \mathrm{O} & -0.022976 & -2.353825 & 0.047663 \\ \mathrm{H} & -0.230986 & -2.914895 & 0.811877 \\ \mathrm{H} & 0.184827 & -2.942367 & -0.694213 \\ \mathrm{O} & -0.163521 & 2.312561 & -0.437099 \\ \mathrm{H} & 0.455696 & 2.745621 & -1.042488 \\ \mathrm{H} & -0.771085 & 2.987511 & -0.091488 \\ \mathrm{O} & -2.507678 & 1.679225 & 0.661927 \\ \mathrm{H} & -3.381071 & 1.921414 & 0.316187 \\ \mathrm{H} & -2.625733 & 1.398958 & 1.590129 \\ \mathrm{O} & -2.857941 & -1.221976 & -0.058224 \\ \mathrm{H} & -3.143318 & -1.383012 & 0.857965 \\ \mathrm{H} & -3.298790 & -1.875910 & -0.621208 \\ \mathrm{O} & -1.286061 & -0.145362 & -2.306832 \\ \mathrm{H} & -0.535533 & -0.083753 & -2.921124 \\ \mathrm{H} & -2.095069 & -0.093872 & -2.838793 \\ \mathrm{O} & -1.263505 & -0.288092 & 2.059383 \\ \mathrm{H} & -1.037829 & -0.424983 & 2.986462\end{array}$

$\left[\left(\mathrm{NH}_{3}{ }^{+} \mathrm{CH}_{2} \mathrm{COO}^{-}\right)^{1} \mathrm{Y}\left(\mathrm{H}_{2} \mathrm{O}\right)_{5}\left(\mathrm{OH}^{-}\right)\right]^{2+}$ (f) $\mathrm{E}=-780.673807$
$\begin{array}{llll}\mathrm{Y} & -0.637811 & -0.000986 & -0.088633\end{array}$
$\begin{array}{llll}\text { C } & 2.146111 & 0.352539 & -0.086690\end{array}$
$\begin{array}{llll}\text { O } & 1.699005 & -0.661942 & 0.549023\end{array}$
$\begin{array}{llll}\text { C } & 3.663470 & 0.452166 & -0.296021\end{array}$
$\begin{array}{llll}\mathrm{O} & 1.407623 & 1.254806 & -0.555962\end{array}$
$\begin{array}{llll}\mathrm{N} & 4.295003 & -0.799933 & 0.279853\end{array}$ 


\begin{tabular}{|c|c|c|c|}
\hline $\mathrm{H}$ & & & \\
\hline $\mathrm{H}$ & & & \\
\hline $\mathrm{H}$ & 3.505708 & & \\
\hline $\mathrm{H}$ & & & \\
\hline $\mathrm{H}$ & .732483 & & \\
\hline $\mathrm{O}$ & & & \\
\hline $\mathrm{H}$ & 1.416793 & -2.55 & \\
\hline $\mathrm{H}$ & & & \\
\hline $\mathrm{O}$ & -0.3 & & \\
\hline $\mathrm{H}$ & & & \\
\hline $\mathrm{H}$ & -0.9 & & \\
\hline $\mathrm{O}$ & -2.68 & & \\
\hline $\mathrm{H}$ & & & \\
\hline $\mathrm{H}$ & -2.96 & -0.1 & \\
\hline $\mathrm{O}$ & -2.48 & & \\
\hline $\mathrm{H}$ & -3.4 & -1.8 & \\
\hline $\mathrm{H}$ & -2.0 & & \\
\hline $\mathrm{O}$ & -1.8 & & \\
\hline $\mathrm{H}$ & -1.8 & 2.6 & \\
\hline $\mathrm{H}$ & -1.8 & & \\
\hline $\mathrm{O}$ & -0.3 & -0.8 & \\
\hline$H$ & 0.0 & -0.9 & \\
\hline \multicolumn{4}{|c|}{$\begin{array}{l}{\left[\left(\mathrm{NH}_{2} \mathrm{CH}_{2} \mathrm{COO}^{-}\right)^{1} \mathrm{Y}\left(\mathrm{H}_{2} \mathrm{O}\right)_{5}(\mathrm{OH})\right]^{+}(\mathrm{a})} \\
\mathrm{E}=-780.4053139\end{array}$} \\
\hline $\mathbf{Y}$ & & & \\
\hline $\mathrm{C}$ & -2.2 & -0.0 & \\
\hline $\mathrm{O}$ & -1.5 & 0.76 & \\
\hline $\mathrm{C}$ & & & \\
\hline $\mathrm{O}$ & -1.6 & -1.0 & \\
\hline $\mathrm{N}$ & -4.2 & & \\
\hline $\mathrm{H}$ & -4.1 & -0.709 & \\
\hline $\mathrm{H}$ & -4.0 & -0.2 & \\
\hline $\mathrm{H}$ & -3.9 & & \\
\hline $\mathrm{H}$ & & & \\
\hline $\mathrm{O}$ & & -1.4 & \\
\hline $\mathrm{H}$ & & -1.1 & \\
\hline $\mathrm{H}$ & & -2.4 & \\
\hline $\mathrm{O}$ & & & \\
\hline $\mathrm{H}$ & 1.88 & 2.0799 & -0.959 \\
\hline $\mathrm{H}$ & 0.40 & 2.3235 & \\
\hline $\mathrm{O}$ & 3.236038 & 1.8775 & \\
\hline $\mathrm{H}$ & 2.593023 & 1.697398 & \\
\hline $\mathrm{H}$ & 3.934015 & 2.467191 & \\
\hline $\mathrm{O}$ & 2.776783 & -0.671292 & -0.381733 \\
\hline $\mathrm{H}$ & 3.307499 & 0.126751 & -0.138559 \\
\hline
\end{tabular}




$\begin{array}{crcc}\mathrm{H} & 3.148019 & -1.036894 & -1.197597 \\ \mathrm{O} & 0.655904 & -2.474971 & 0.920888 \\ \mathrm{H} & 1.407005 & -2.892508 & 1.368989 \\ \mathrm{H} & -0.162113 & -2.769392 & 1.356898 \\ \mathrm{O} & 1.199347 & 0.883592 & 1.723549 \\ \mathrm{H} & 0.971182 & 1.117854 & 2.631046\end{array}$

\begin{tabular}{cccc}
\multicolumn{5}{c}{$\left[\left(\mathrm{NH}_{2} \mathrm{CH}_{2} \mathrm{COO}^{-}\right)\right.$} & $\left.{ }^{1} \mathrm{Y}\left(\mathrm{H}_{2} \mathrm{O}\right)_{5}(\mathrm{OH})\right]^{+}(b)$ \\
$\mathrm{E}=-780.4052603$ \\
$\mathrm{Y}$ & 0.585489 & 0.013632 & -0.062008 \\
$\mathrm{O}$ & 1.600568 & -0.693728 & -1.815121 \\
$\mathrm{O}$ & -1.272262 & 1.057491 & -1.113753 \\
$\mathrm{H}$ & 1.503333 & -0.723557 & -2.773880 \\
$\mathrm{O}$ & -1.417373 & 0.684467 & 1.067285 \\
$\mathrm{C}$ & -1.978051 & 0.845241 & -0.073464 \\
$\mathrm{C}$ & -3.463308 & 0.535264 & -0.199021 \\
$\mathrm{~N}$ & -3.582061 & -0.922698 & 0.101362 \\
$\mathrm{H}$ & -3.783144 & 0.721622 & -1.223799 \\
$\mathrm{H}$ & -4.046551 & 1.157034 & 0.487147 \\
$\mathrm{H}$ & -3.706953 & -1.054497 & 1.103619 \\
$\mathrm{H}$ & -4.410202 & -1.303738 & -0.351425 \\
$\mathrm{O}$ & 1.069070 & 2.370711 & -0.224359 \\
$\mathrm{H}$ & 1.985330 & 2.674342 & -0.317973 \\
$\mathrm{H}$ & 0.483600 & 2.964875 & -0.717847 \\
$\mathrm{O}$ & -1.002786 & -1.831400 & -0.350000 \\
$\mathrm{H}$ & -0.918282 & -2.332927 & -1.174502 \\
$\mathrm{H}$ & -1.990376 & -1.646469 & -0.222588 \\
$\mathrm{O}$ & 0.848876 & 0.111932 & 2.351435 \\
$\mathrm{H}$ & 1.494890 & -0.067812 & 3.048038 \\
$\mathrm{H}$ & 0.049114 & 0.492488 & 2.752522 \\
$\mathrm{O}$ & 3.074587 & 0.577059 & -0.062101 \\
$\mathrm{H}$ & 3.805088 & 0.216234 & 0.460828 \\
$\mathrm{H}$ & 3.078723 & 0.123118 & -0.938819 \\
$\mathrm{O}$ & 1.831964 & -2.080620 & 0.495615 \\
$\mathrm{H}$ & 1.422700 & -2.880563 & 0.858181 \\
$\mathrm{H}$ & 2.060095 & -2.263248 & -0.437836
\end{tabular}

$\left[\left(\mathrm{NH}_{2} \mathrm{CH}_{2} \mathrm{COO}^{-}\right){ }^{1} \mathrm{Y}\left(\mathrm{H}_{2} \mathrm{O}\right)_{5}(\mathrm{OH})\right]^{+}(\mathrm{c})$ $\mathrm{E}=-780.4067159$
$\begin{array}{llll}\mathrm{Y} & -0.596596 & 0.083593 & 0.052497\end{array}$
C $\quad 1.925512 \quad-0.829492 \quad-0.222520$
$\begin{array}{llll}\text { O } & 1.285695 & -1.051242 & 0.867267\end{array}$
$\begin{array}{llll}\text { C } & 3.430209 & -0.603319 & -0.157723\end{array}$
$\begin{array}{llll}\text { O } & 1.293819 & -0.600245 & -1.306424\end{array}$
$\begin{array}{llll}\mathrm{N} & 3.595158 & 0.740131 & 0.475967\end{array}$
H $\quad 3.916689-1.398318 \quad 0.414056$ 


\begin{tabular}{|c|c|c|c|}
\hline $\mathrm{H}$ & & & \\
\hline $\mathrm{H}$ & & & \\
\hline $\mathrm{H}$ & 437059 & & \\
\hline $\mathrm{O}$ & & & \\
\hline $\mathrm{H}$ & & -2.714242 & \\
\hline $\mathrm{H}$ & & & \\
\hline $\mathrm{O}$ & & -1.458744 & \\
\hline $\mathrm{H}$ & & & \\
\hline $\mathrm{H}$ & -0.899 & -2.0 & \\
\hline $\mathrm{O}$ & 1.000 & 1.8 & \\
\hline $\mathrm{H}$ & & & \\
\hline $\mathrm{H}$ & 0.88 & & \\
\hline $\mathrm{O}$ & & & \\
\hline $\mathrm{H}$ & -3.0 & & \\
\hline $\mathrm{H}$ & & & \\
\hline $\mathrm{O}$ & -0.9 & & \\
\hline $\mathrm{H}$ & -0.2 & & \\
\hline $\mathrm{H}$ & & & \\
\hline $\mathrm{O}$ & -1.5 & & \\
\hline $\mathrm{H}$ & -1.5 & & \\
\hline \multicolumn{4}{|c|}{$\begin{array}{l}{\left[\left(\mathrm{NH}_{2} \mathrm{CH}_{2} \mathrm{COO}^{-}\right){ }^{1} \mathrm{Y}\left(\mathrm{H}_{2} \mathrm{O}\right)_{5}(\mathrm{OH})\right]^{+}(\mathrm{d})} \\
\mathrm{E}=-780.4067923\end{array}$} \\
\hline & & & \\
\hline $\mathrm{C}$ & & & \\
\hline $\mathrm{O}$ & 1.3 & -0. & \\
\hline C & & & \\
\hline $\mathrm{O}$ & 1.2 & -1 . & \\
\hline $\mathrm{N}$ & & & \\
\hline $\mathrm{H}$ & 3.98 & -1.0 & \\
\hline $\mathrm{H}$ & 3.7 & -0.6 & \\
\hline $\mathrm{H}$ & & 1.2 & \\
\hline $\mathrm{H}$ & & & \\
\hline $\mathrm{O}$ & -0.9 & -2. & \\
\hline $\mathrm{H}$ & -1.7 & -2. & \\
\hline $\mathrm{H}$ & -0.2 & -3.0 & \\
\hline $\mathrm{O}$ & -0.87 & & \\
\hline $\mathrm{H}$ & -1.68 & 0.3 & \\
\hline $\mathrm{H}$ & -0.11 & 0.2 & \\
\hline $\mathrm{O}$ & 0.849 & 1.819333 & -0.7 \\
\hline $\mathrm{H}$ & 1.845 & 1.707092 & -0.2 \\
\hline $\mathrm{H}$ & 0.682384 & & \\
\hline $\mathrm{O}$ & -1.965674 & 2.071487 & -0.3 \\
\hline $\mathrm{H}$ & -2.771031 & 1.531429 & -0.158768 \\
\hline $\mathrm{H}$ & -1.998527 & 2.890484 & 0.152044 \\
\hline $\mathrm{O}$ & -0.880326 & -0.156373 & -2.388313 \\
\hline
\end{tabular}




\begin{tabular}{|c|c|c|c|}
\hline H & 0.142800 & & \\
\hline $\mathrm{H}$ & -1.613066 & & \\
\hline $\mathrm{O}$ & -2.828393 & -0.244827 & \\
\hline H & -3.633483 & -0.770810 & 0.19 \\
\hline \multicolumn{4}{|c|}{$\begin{array}{l}{\left[\left(\mathrm{NH}_{2} \mathrm{CH}_{2} \mathrm{COO}^{-}\right)^{1} \mathrm{Y}\left(\mathrm{H}_{2} \mathrm{O}\right)_{5}(\mathrm{OH})\right]^{+}(\mathrm{e})} \\
\mathrm{E}=-780.4039327\end{array}$} \\
\hline Y & 0.586590 & -0.016625 & -0.13309 \\
\hline & & & \\
\hline & -1.4 & -0.79 & \\
\hline & & -0.09 & \\
\hline & -1.2 & -1. & \\
\hline & -1.9 & & \\
\hline & -3.4 & -0. & \\
\hline $\mathbf{N}$ & -3.5 & & \\
\hline $\mathrm{H}$ & -3.7 & -0.3 & \\
\hline $\mathrm{H}$ & -4.0 & -1. & \\
\hline $\mathrm{H}$ & -3.5 & & \\
\hline $\mathrm{H}$ & -4.3 & & -0 . \\
\hline $\mathrm{O}$ & & & \\
\hline $\mathrm{H}$ & 1.3 & & \\
\hline $\mathrm{H}$ & -0.1 & & \\
\hline $\mathrm{O}$ & & & \\
\hline $\mathrm{H}$ & & & \\
\hline $\mathrm{H}$ & & & \\
\hline $\mathrm{O}$ & 1.0 & -2.4 & \\
\hline $\mathrm{H}$ & & -2.9 & \\
\hline $\mathrm{H}$ & 1.1 & -2.7 & \\
\hline $\mathrm{O}$ & & -0.5 & \\
\hline H & 3.4 & & \\
\hline $\mathrm{H}$ & 3.2 & -1.3 & \\
\hline $\mathrm{O}$ & -0.93 & & -0. \\
\hline $\mathrm{H}$ & -0.8 & 2.6 & \\
\hline H & -1.93 & 203 & -0.1 \\
\hline \multirow{2}{*}{\multicolumn{4}{|c|}{$\begin{array}{l}{\left[\left(\mathrm{NH}_{2} \mathrm{CH}_{2} \mathrm{COO}^{-}\right)^{1} \mathrm{Y}\left(\mathrm{H}_{2} \mathrm{O}\right)_{5}(\mathrm{OH})\right]^{+}(\mathrm{f})} \\
\mathrm{E}=-780.4017958\end{array}$}} \\
\hline & & & \\
\hline $\mathrm{Y}$ & -0.607617 & -0.019113 & -0.165989 \\
\hline & -1.58 & -0.66 & \\
\hline $\mathrm{C}$ & & -0.143636 & \\
\hline & -1.342014 & -1.023434 & -2.77 \\
\hline $\mathrm{C}$ & 1.1096 & -1.52 & 0.40 \\
\hline & 1.908983 & -0.883471 & -0.36 \\
\hline $\mathrm{C}$ & 3.396578 & -0.810229 & -0.05 \\
\hline $\mathrm{N}$ & 3.676087 & 0.625660 & 0.233838 \\
\hline $\mathrm{H}$ & 3.976748 & -1.195457 & -0.90155 \\
\hline
\end{tabular}




\begin{tabular}{|c|c|c|c|}
\hline $\mathrm{H}$ & & & \\
\hline $\mathrm{H}$ & & 0.706761 & \\
\hline $\mathrm{H}$ & 3.854128 & 1.117441 & -0.640447 \\
\hline $\mathrm{O}$ & & 2.294954 & \\
\hline $\mathrm{H}$ & .020080 & 2.584996 & \\
\hline $\mathrm{H}$ & & & \\
\hline $\mathrm{O}$ & & & \\
\hline $\mathrm{H}$ & 1.041656 & & \\
\hline $\mathrm{H}$ & & & \\
\hline $\mathrm{O}$ & 31 & -1.9 & 69 \\
\hline $\mathrm{H}$ & -2.32 & & \\
\hline $\mathrm{H}$ & -0.8 & -2.6 & 785 \\
\hline $\mathrm{O}$ & & & \\
\hline $\mathrm{H}$ & -3.9 & & \\
\hline $\mathrm{H}$ & -3.0 & -0.2 & 79 \\
\hline $\mathrm{O}$ & -1.3 & & \\
\hline $\mathrm{H}$ & & & \\
\hline $\mathrm{H}$ & -1.6 & -0.0 & \\
\hline \multicolumn{4}{|c|}{$\left[\left(\mathrm{NH}_{3}{ }^{+} \mathrm{CH}_{2} \mathrm{COO}^{-}\right){ }^{1} \mathrm{La}\left(\mathrm{H}_{2} \mathrm{O}\right)_{7}\right]^{3}$} \\
\hline \multicolumn{4}{|c|}{$\mathrm{E}=-1178.2740212$} \\
\hline & -0.640578 & & \\
\hline & & & \\
\hline $\mathrm{O}$ & & & \\
\hline $\mathrm{C}$ & 2.22 & -0.3 & \\
\hline $\mathrm{C}$ & 3.7 & -0.7 & \\
\hline$\Lambda$ & & & \\
\hline $\mathrm{H}$ & 3.90 & -1.5 & \\
\hline $\mathrm{H}$ & & & \\
\hline $\mathrm{H}$ & 4.28 & & \\
\hline $\mathrm{H}$ & $5.5^{\circ}$ & & \\
\hline $\mathrm{H}$ & & & \\
\hline $\mathrm{O}$ & -0.6 & & \\
\hline $\mathrm{H}$ & & & \\
\hline $\mathrm{H}$ & & & \\
\hline $\mathrm{O}$ & -1.97 & -0.9 & \\
\hline $\mathrm{H}$ & & -0.4 & \\
\hline $\mathrm{H}$ & -2.1085 & -1.881 & -2. \\
\hline $\mathrm{O}$ & -3.12 & 0.2 & \\
\hline $\mathrm{H}$ & -3.920315 & 0.229 & \\
\hline $\mathrm{H}$ & -3.46 & 0.37 & \\
\hline $\mathrm{O}$ & -0.55 & -0.23 & \\
\hline $\mathrm{H}$ & -0.613661 & -1.077488 & .7776 \\
\hline $\mathrm{H}$ & & 0.442222 & 3.20 \\
\hline $\mathrm{O}$ & -1.074993 & -2.477009 & 0.433049 \\
\hline $\mathrm{H}$ & -1.844420 & -3.001676 & 0.716314 \\
\hline
\end{tabular}




\begin{tabular}{cccc}
$\mathrm{H}$ & -0.348097 & -3.111783 & 0.296750 \\
$\mathrm{O}$ & -0.419872 & 1.584478 & -2.099815 \\
$\mathrm{H}$ & -0.405358 & 2.557289 & -2.067415 \\
$\mathrm{H}$ & -0.242077 & 1.354670 & -3.028898 \\
\multicolumn{4}{c}{} \\
{$\left[\mathrm{NH}_{3}{ }^{+} \mathrm{CH}_{2} \mathrm{COO}^{-}\right)$} & $\left.{ }^{1} \mathrm{La}\left(\mathrm{H}_{2} \mathrm{O}\right)_{6}\left(\mathrm{OH}^{-}\right)\right]^{2+}(\mathrm{a})$ \\
$\mathrm{E}=-1254.600714$ & & \\
$\mathrm{LA}$ & -0.670379 & 0.009344 & -0.144487 \\
$\mathrm{O}$ & -0.772383 & 0.340666 & -2.234727 \\
$\mathrm{H}$ & -0.775541 & 0.619945 & -3.158847 \\
$\mathrm{C}$ & 2.298814 & -0.378795 & 0.151744 \\
$\mathrm{C}$ & 3.797854 & -0.723822 & 0.142028 \\
$\mathrm{O}$ & 1.973747 & 0.823691 & -0.109157 \\
$\mathrm{O}$ & 1.480460 & -1.304951 & 0.403921 \\
$\mathrm{~N}$ & 4.549165 & 0.524877 & -0.273046 \\
$\mathrm{H}$ & 4.000261 & -1.530179 & -0.562458 \\
$\mathrm{H}$ & 4.122526 & -1.033777 & 1.135578 \\
$\mathrm{H}$ & 5.028408 & 0.416946 & -1.171539 \\
$\mathrm{H}$ & 3.806046 & 1.251470 & -0.367476 \\
$\mathrm{H}$ & 5.240507 & 0.825523 & 0.419655 \\
$\mathrm{O}$ & -3.226815 & 0.617127 & -0.061478 \\
$\mathrm{H}$ & -3.819269 & 0.858351 & 0.667678 \\
$\mathrm{H}$ & -3.779632 & 0.566249 & -0.858207 \\
$\mathrm{O}$ & -1.541988 & -2.197653 & -1.216882 \\
$\mathrm{H}$ & -1.730940 & -3.120177 & -0.989734 \\
$\mathrm{H}$ & -1.558591 & -2.123647 & -2.185008 \\
$\mathrm{O}$ & -0.469475 & 2.686753 & -0.025019 \\
$\mathrm{H}$ & -1.058975 & 3.261983 & -0.539131 \\
$\mathrm{H}$ & 0.429297 & 3.013201 & -0.192498 \\
$\mathrm{O}$ & -0.969326 & -1.887286 & 1.795828 \\
$\mathrm{H}$ & -1.670602 & -2.381592 & 2.247510 \\
$\mathrm{H}$ & -0.149138 & -2.393979 & 1.917854 \\
$\mathrm{O}$ & -1.010298 & 1.099479 & 2.259786 \\
$\mathrm{H}$ & -0.962690 & 2.061132 & 2.382774 \\
$\mathrm{H}$ & -1.045586 & 0.694872 & 3.140126 \\
& &
\end{tabular}

$\left[\left(\mathrm{NH}_{3}{ }^{+} \mathrm{CH}_{2} \mathrm{COO}^{-}\right){ }^{1} \mathrm{La}\left(\mathrm{H}_{2} \mathrm{O}\right)_{6}\left(\mathrm{OH}^{-}\right)\right]^{2+}$ (b) $\mathrm{E}=-1254.600142$
LA $\quad-0.774831 \quad-0.057919 \quad 0.000904$
$\begin{array}{llll}\mathrm{O} & 1.420341 & 1.341484 & -0.031140\end{array}$
$\begin{array}{llll}\mathrm{O} & 1.925629 & -0.851616 & 0.012913\end{array}$
$\begin{array}{llll}\text { C } & 2.239022 & 0.380617 & -0.010934\end{array}$
$\begin{array}{llll}\text { C } & 3.743272 & 0.715258 & -0.014491\end{array}$
$\begin{array}{llll}\mathrm{N} & 4.491213 & -0.601722 & 0.007636\end{array}$
$\mathrm{H} \quad 4.006551 \quad 1.304983 \quad 0.863433$
H $\quad 4.009370 \quad 1.277339 \quad-0.909491$ 


\begin{tabular}{|c|c|c|c|}
\hline $\mathrm{H}$ & & & \\
\hline $\mathrm{H}$ & & & \\
\hline 11 & & & \\
\hline $\mathrm{O}$ & & & \\
\hline $\mathrm{H}$ & & & \\
\hline $\mathrm{H}$ & & & \\
\hline $\mathrm{O}$ & & & \\
\hline $\mathrm{H}$ & & & \\
\hline $\mathrm{H}$ & & -3. & \\
\hline $\mathrm{O}$ & & & \\
\hline $\mathrm{H}$ & & & \\
\hline $\mathrm{H}$ & 0 & & \\
\hline $\mathrm{O}$ & & & \\
\hline $\mathrm{H}$ & & & \\
\hline $\mathrm{H}$ & & & \\
\hline $\mathrm{O}$ & & & \\
\hline $\mathrm{H}$ & & & \\
\hline $\mathrm{H}$ & -0.5 & & \\
\hline $\mathrm{O}$ & -2.8 & & \\
\hline $\mathrm{H}$ & -3.8 & -0. & \\
\hline \multicolumn{4}{|c|}{$\begin{array}{l}\left.\left(\mathrm{NH}_{3}{ }^{+} \mathrm{CH}_{2} \mathrm{COO}^{-}\right){ }^{1} \mathrm{La}\left(\mathrm{H}_{2} \mathrm{O}\right)_{6}\left(\mathrm{OH}^{-}\right)\right]^{2+}(\mathrm{c}) \\
\mathrm{E}=-1254.592782\end{array}$} \\
\hline & & & \\
\hline & -0.5 & & \\
\hline & & & \\
\hline $\mathrm{H}$ & -0.9 & & \\
\hline $\mathrm{H}$ & -0.1 & -2. & \\
\hline $\mathrm{O}$ & & & \\
\hline $\mathrm{O}$ & -3.2 & -0.9 & \\
\hline $\mathrm{O}$ & & & \\
\hline $\mathrm{O}$ & & & \\
\hline $\mathrm{O}$ & -2.0 & & \\
\hline $\mathrm{O}$ & & -0.8 & \\
\hline $\mathrm{H}$ & & & \\
\hline $\mathrm{H}$ & 0.22 & -2.4 & \\
\hline $\mathrm{H}$ & -1.79 & & \\
\hline $\mathrm{H}$ & -0.7 & & \\
\hline $\mathrm{H}$ & -3.92 & -0.9 & \\
\hline $\mathrm{H}$ & -3.695 & -1.2 & \\
\hline $\mathrm{H}$ & -0.93 & & \\
\hline $\mathrm{H}$ & -1.760398 & 2.935606 & -0.8 \\
\hline $\mathrm{C}$ & 2.607551 & 0.094049 & -0.0 \\
\hline $\mathrm{H}$ & -2.471020 & & -2.741499 \\
\hline $\mathrm{C}$ & 3.840901 & 0.793713 & -0.645865 \\
\hline $\mathrm{N}$ & 5.066689 & 0.107176 & -0.080515 \\
\hline
\end{tabular}




\begin{tabular}{|c|c|c|c|}
\hline $\mathrm{H}$ & & & \\
\hline $\mathrm{H}$ & & & \\
\hline & & & \\
\hline $\mathrm{H}$ & & & \\
\hline & & & \\
\hline \multicolumn{4}{|c|}{$\begin{array}{l}{\left[\left(\mathrm{NH}_{3}{ }^{+} \mathrm{CH}_{2} \mathrm{COO}^{-}\right){ }^{1} \mathrm{La}\left(\mathrm{H}_{2} \mathrm{O}\right)_{6}\left(\mathrm{OH}^{-}\right)\right]^{2+}(\mathrm{d})} \\
\mathrm{E}=-1254.600812\end{array}$} \\
\hline & 0.897704 & 0.099687 & -0.011400 \\
\hline & 2.928 & 0.8 & \\
\hline & 3.7 & & \\
\hline & -2.7 & -0.0 & \\
\hline & -4.0 & & \\
\hline 0 & -2.8 & -1. & \\
\hline $\mathrm{O}$ & -1.6 & & \\
\hline iv & -5.1 & -0.3 & \\
\hline $\mathrm{H}$ & & & \\
\hline $\mathrm{H}$ & & & \\
\hline $\mathrm{H}$ & -5.7 & & \\
\hline $\mathrm{H}$ & -4.6 & & \\
\hline $\mathrm{H}$ & -5.7 & -0.5 & \\
\hline $\mathrm{O}$ & & & \\
\hline $\mathrm{H}$ & -0.1 & -3 . & \\
\hline $\mathrm{H}$ & -1.2 & -1. & \\
\hline $\mathrm{O}$ & 0.4 & & \\
\hline $\mathrm{H}$ & & & \\
\hline $\mathrm{H}$ & & & \\
\hline $\mathrm{O}$ & 2.1 & -0.8 & \\
\hline $\mathrm{H}$ & & & \\
\hline $\mathrm{H}$ & 3.0 & -0.6 & \\
\hline $\mathrm{O}$ & & -0.3 & \\
\hline $\mathrm{H}$ & & -0.9 & \\
\hline $\mathrm{H}$ & 0.0 & & -3.3 \\
\hline $\mathrm{O}$ & & -1.7 & \\
\hline $\mathrm{H}$ & & & \\
\hline $\mathrm{H}$ & 3.25 & -1.1 & \\
\hline $\mathrm{O}$ & 0.3 & & \\
\hline $\mathrm{H}$ & $-0.4 c$ & & -1.0 \\
\hline $\mathrm{H}$ & 1.074307 & 3.164771 & \\
\hline \multicolumn{4}{|c|}{$\left[\left(\mathrm{NH}_{3}{ }^{+} \mathrm{CH}_{2} \mathrm{COO}^{-}\right){ }^{1} \mathrm{La}\left(\mathrm{H}_{2} \mathrm{O}\right)_{6}\left(\mathrm{OH}^{-}\right)\right]^{2+}(\mathrm{e})$} \\
\hline \multicolumn{4}{|c|}{$E=-1254.602128$} \\
\hline & 0.808910 & & 0.089881 \\
\hline $\mathrm{C}$ & -2.735912 & 0.08 & -0.061455 \\
\hline $\mathrm{O}$ & -1.588451 & 0.585505 & -0.258211 \\
\hline $\mathrm{C}$ & -3.942762 & 1.042195 & -0.206666 \\
\hline
\end{tabular}




\begin{tabular}{|c|c|c|c|}
\hline $\mathrm{O}$ & & & \\
\hline $\mathrm{N}$ & & & \\
\hline $\mathrm{H}$ & & & \\
\hline $\mathrm{H}$ & & & \\
\hline $\mathrm{H}$ & & & \\
\hline $\mathrm{H}$ & & & \\
\hline $\mathrm{H}$ & 8054 & -0.7 & \\
\hline $\mathrm{O}$ & & & \\
\hline $\mathrm{H}$ & & & \\
\hline $\mathrm{H}$ & & & \\
\hline $\mathrm{O}$ & & & \\
\hline $\mathrm{H}$ & & -1.7 & \\
\hline $\mathrm{H}$ & & -2.5 & \\
\hline $\mathrm{O}$ & & -1.8 & \\
\hline $\mathrm{H}$ & & -1.5 & \\
\hline $\mathrm{H}$ & & & \\
\hline $\mathrm{O}$ & & & \\
\hline $\mathrm{H}$ & & & \\
\hline $\mathrm{H}$ & & & \\
\hline $\mathrm{O}$ & & & \\
\hline $\mathrm{H}$ & & & \\
\hline $\mathrm{H}$ & & & \\
\hline $\mathrm{O}$ & & -2.2 & \\
\hline $\mathrm{H}$ & -1.5 & -2.0 & \\
\hline $\mathrm{H}$ & & & \\
\hline $\mathrm{O}$ & & & \\
\hline$H$ & & $-0 .($ & \\
\hline \multicolumn{4}{|c|}{$\begin{array}{l}{\left[\left(\mathrm{NH}_{3}{ }^{+} \mathrm{CH}_{2} \mathrm{COO}^{-}\right)^{1}{ }^{1} \mathrm{La}\left(\mathrm{H}_{2} \mathrm{O}\right)_{6}\left(\mathrm{OH}^{-}\right)\right]^{2+}(\mathrm{f})} \\
\mathrm{E}=-1254.596678\end{array}$} \\
\hline & & & \\
\hline C & -2.7 & $-0 .($ & \\
\hline $\mathrm{O}$ & -1.6 & -0. & \\
\hline $\mathrm{C}$ & -3.9 & & \\
\hline O & -2.9 & & \\
\hline $\mathrm{N}$ & -5.1 & -0.1 & \\
\hline $\mathrm{H}$ & -3.8 & -1.7 & \\
\hline $\mathrm{H}$ & -4.1 & & \\
\hline $\mathrm{H}$ & -5.5 & -0.363569 & -1.2 \\
\hline $\mathrm{H}$ & -5.8 & -0.1 & \\
\hline $\mathrm{H}$ & -4.7 & & \\
\hline $\mathrm{O}$ & & -0.092860 & \\
\hline $\mathrm{H}$ & -0.494134 & -0.281759 & \\
\hline & & & \\
\hline $\mathrm{O}$ & 2.113929 & 2.154406 & -0.811730 \\
\hline $\mathrm{H}$ & 2.764491 & 2.188779 & -1.530908 \\
\hline
\end{tabular}




\begin{tabular}{|c|c|c|c|}
\hline $\mathrm{H}$ & & & \\
\hline $\mathrm{O}$ & & & \\
\hline $\mathrm{H}$ & & & \\
\hline $\mathrm{H}$ & & & \\
\hline $\mathrm{O}$ & & & \\
\hline $\mathrm{H}$ & 495 & & \\
\hline $\mathrm{H}$ & & & \\
\hline $\mathrm{O}$ & & 72 & \\
\hline $\mathrm{H}$ & & & \\
\hline $\mathrm{H}$ & 0.9 & 41 & \\
\hline $\mathrm{O}$ & & & \\
\hline $\mathrm{H}$ & & 2.0 & \\
\hline $\mathrm{H}$ & & & \\
\hline $\mathrm{O}$ & & -0.2 & \\
\hline $\mathrm{H}$ & & ? & \\
\hline \multicolumn{4}{|c|}{$\begin{array}{l}{\left[\left(\mathrm{NH}_{3}{ }^{+} \mathrm{CH}_{2} \mathrm{COO}^{-}\right){ }^{1} \mathrm{La}\left(\mathrm{H}_{2} \mathrm{O}\right)_{6}\left(\mathrm{OH}^{-}\right)\right]^{2+}(\mathrm{g}} \\
\mathrm{E}=-1254.59078\end{array}$} \\
\hline & 0.601190 & 0.003069 & \\
\hline & -2.38 & & \\
\hline$U$ & -1.6 & -0. & \\
\hline & -3.9 & & \\
\hline $\mathrm{O}$ & -1.9 & & \\
\hline $\mathrm{N}$ & -4.5 & & \\
\hline $\mathrm{H}$ & & & \\
\hline $\mathrm{H}$ & -4.2 & -1.3 & \\
\hline $\mathrm{H}$ & -5.1 & & \\
\hline $\mathrm{H}$ & -5.0 & -0 & \\
\hline $\mathrm{H}$ & -3.6 & & \\
\hline $\mathrm{O}$ & -0.0 & & \\
\hline $\mathrm{H}$ & & & \\
\hline $\mathrm{H}$ & -0.0 & & -1 . \\
\hline $\mathrm{O}$ & -0.0 & -2.4 & \\
\hline $\mathrm{H}$ & -0.1 & & \\
\hline $\mathrm{H}$ & & -2.63 & \\
\hline $\mathrm{O}$ & $1.2^{\prime}$ & 2.4 & \\
\hline $\mathrm{H}$ & 1.9 & 3.1 & \\
\hline $\mathrm{H}$ & 1.3( & & \\
\hline $\mathrm{O}$ & 2.96 & -1.01 & \\
\hline $\mathrm{H}$ & 2.69 & -0.76 & \\
\hline $\mathrm{H}$ & 3.52 & -1.79 & \\
\hline $\mathrm{O}$ & 2.78 & 0.58 & -1.3 \\
\hline $\mathrm{H}$ & 3.615288 & 0.370398 & -0.85 \\
\hline $\mathrm{H}$ & 3.00 & & -2.0 \\
\hline $\mathrm{O}$ & 0.760021 & -1.688488 & -2.002711 \\
\hline $\mathrm{H}$ & 1.468339 & -1.980688 & -2.596106 \\
\hline
\end{tabular}




$\begin{array}{cccc}\mathrm{H} & -0.085897 & -1.860780 & -2.447576 \\ \mathrm{O} & 0.922814 & 0.149031 & 2.241006 \\ \mathrm{H} & 0.564819 & 0.117758 & 3.137534\end{array}$

$\left[\left(\mathrm{NH}_{2} \mathrm{CH}_{2} \mathrm{COO}^{-}\right){ }^{1} \mathrm{La}\left(\mathrm{H}_{2} \mathrm{O}\right)_{6}(\mathrm{OH})\right]^{+}+\mathrm{H}^{+}$(a) $\mathrm{E}=-1254.310818$

$\begin{array}{lrrr}\text { LA } & 0.673404 & -0.232533 & -0.150741 \\ \mathrm{C} & -2.260911 & 0.007762 & 0.009678 \\ \mathrm{O} & -1.533784 & 1.033771 & 0.275490 \\ \mathrm{C} & -3.781422 & 0.141798 & 0.059761 \\ \mathrm{O} & -1.700889 & -1.100183 & -0.295003 \\ \mathrm{~N} & -4.580211 & -1.029096 & -0.248337 \\ \mathrm{H} & -4.031476 & 0.510109 & 1.061675 \\ \mathrm{H} & -4.044089 & 0.959396 & -0.622206 \\ \mathrm{H} & -4.395515 & -1.786096 & 0.403262 \\ \mathrm{H} & -4.382833 & -1.375862 & -1.182645 \\ \mathrm{O} & -0.583875 & 3.499541 & 0.259982 \\ \mathrm{H} & -1.285299 & 2.811036 & 0.339346 \\ \mathrm{H} & -0.983943 & 4.364620 & 0.428021 \\ \mathrm{O} & 0.746231 & -0.973752 & 2.375583 \\ \mathrm{H} & 0.766143 & -0.410570 & 3.163126 \\ \mathrm{H} & 0.606128 & -1.890077 & 2.658478 \\ \mathrm{O} & 3.314424 & -0.345672 & 0.193427 \\ \mathrm{H} & 4.053830 & -0.701033 & 0.704869 \\ \mathrm{H} & 3.356027 & -0.708568 & -0.719036 \\ \mathrm{O} & 1.300379 & 1.826592 & 1.398438 \\ \mathrm{H} & 2.214004 & 2.143676 & 1.452233 \\ \mathrm{H} & 0.734165 & 2.608330 & 1.198943 \\ \mathrm{O} & 0.651792 & 1.896422 & -1.630627 \\ \mathrm{H} & 0.244049 & 2.720904 & -1.292616 \\ \mathrm{H} & 1.048910 & 2.064602 & -2.495724 \\ \mathrm{O} & 0.280923 & -2.862702 & 0.289431 \\ \mathrm{H} & -0.667458 & -2.906510 & 0.068318 \\ \mathrm{H} & 0.727654 & -3.533811 & -0.248296 \\ \mathrm{O} & 1.783996 & -0.980405 & -1.891247 \\ \mathrm{H} & 1.897588 & -1.258380 & -2.807552\end{array}$

$\left[\left(\mathrm{NH}_{2} \mathrm{CH}_{2} \mathrm{COO}^{-}\right){ }^{1} \mathrm{La}\left(\mathrm{H}_{2} \mathrm{O}\right)_{6}(\mathrm{OH})\right]^{+}+\mathrm{H}^{+}(\mathrm{b})$ $\mathrm{E}=-1254.309212$
$\begin{array}{llll}\text { LA } & 0.389200 & 0.030720 & -0.144650\end{array}$
$\begin{array}{llll}\mathrm{C} & -2.522805 & 0.183790 & -0.112330\end{array}$
$\begin{array}{llll}\mathrm{O} & -1.794059 & 1.026252 & -0.761224\end{array}$
$\begin{array}{llll}\text { C } & -4.039656 & 0.362026 & -0.139723\end{array}$
$\begin{array}{llll}\mathrm{O} & -1.972181 & -0.765165 & 0.541102\end{array}$
$\begin{array}{llll}\mathrm{N} & -4.855969 & -0.632699 & 0.533101\end{array}$
$\mathrm{H} \quad-4.241307 \quad 1.355200 \quad 0.280213$ 


\begin{tabular}{|c|c|c|c|}
\hline $\mathrm{H}$ & -4.331803 & 0.434368 & -1.193090 \\
\hline $\mathrm{H}$ & -4.585742 & -0.735145 & 1.507083 \\
\hline $\mathrm{H}$ & -4.760100 & -1.545509 & 0.097786 \\
\hline $\mathrm{O}$ & 0.391854 & 2.439488 & -1.210019 \\
\hline $\mathrm{H}$ & -0.556206 & 2.591032 & -1.379818 \\
\hline $\mathrm{H}$ & 0.890928 & 2.819521 & -1.946981 \\
\hline $\mathrm{O}$ & -0.427559 & -2.143899 & -1.316341 \\
\hline $\mathrm{H}$ & 0.079918 & -2.821618 & -1.785114 \\
\hline $\mathrm{H}$ & -1.232255 & -2.552460 & -0.960596 \\
\hline $\mathrm{O}$ & -0.140747 & 0.784210 & 2.330117 \\
\hline $\mathrm{H}$ & 0.500320 & 1.222407 & 2.909479 \\
\hline $\mathrm{H}$ & -0.947957 & 0.616671 & 2.838232 \\
\hline $\mathrm{O}$ & 3.971902 & -0.601711 & 0.183141 \\
\hline $\mathrm{H}$ & 4.896694 & -0.802166 & -0.017476 \\
\hline $\mathrm{H}$ & 3.443368 & -0.563578 & -0.676237 \\
\hline $\mathrm{O}$ & 2.352736 & 1.401335 & 1.068867 \\
\hline $\mathrm{H}$ & 3.180635 & 0.892475 & 0.896497 \\
\hline $\mathrm{H}$ & 2.534893 & 2.323833 & 0.836926 \\
\hline $\mathrm{O}$ & 1.753709 & -1.712113 & 1.262504 \\
\hline $\mathrm{H}$ & 1.661647 & -2.171371 & 2.108131 \\
\hline $\mathrm{H}$ & 2.714597 & -1.611904 & 1.065193 \\
\hline $\mathrm{O}$ & 2.074495 & -0.371488 & -1.600017 \\
\hline $\mathrm{H}$ & 2.253292 & -0.504092 & -2.539632 \\
\hline \multicolumn{4}{|c|}{$\begin{array}{l}{\left[\left(\mathrm{NH}_{2} \mathrm{CH}_{2} \mathrm{COO}^{-}\right){ }^{1} \mathrm{La}\left(\mathrm{H}_{2} \mathrm{O}\right)_{6}(\mathrm{OH})\right]^{+}+\mathrm{H}^{+}(\mathrm{c}} \\
\mathrm{E}=-1254.309428\end{array}$} \\
\hline LA & 0.387536 & -0.012791 & -0.091955 \\
\hline $\mathrm{O}$ & 1.913033 & -0.080634 & -1.755675 \\
\hline $\mathrm{H}$ & 1.979029 & -0.048906 & -2.718629 \\
\hline $\mathrm{C}$ & -2.518400 & 0.291749 & 0.154514 \\
\hline $\mathrm{C}$ & -4.035638 & 0.440773 & 0.265199 \\
\hline $\mathrm{O}$ & -1.762200 & 1.180978 & 0.686206 \\
\hline $\mathrm{O}$ & -1.998205 & -0.704718 & -0.464678 \\
\hline $\mathrm{N}$ & -4.869500 & -0.582633 & -0.337641 \\
\hline $\mathrm{H}$ & -4.267589 & 0.528982 & 1.332905 \\
\hline $\mathrm{H}$ & -4.284162 & 1.420327 & -0.159959 \\
\hline $\mathrm{H}$ & -4.698065 & -1.493267 & 0.078523 \\
\hline $\mathrm{H}$ & -4.690804 & -0.664793 & -1.334330 \\
\hline $\mathrm{O}$ & 2.239802 & -1.472542 & 1.127564 \\
\hline $\mathrm{H}$ & 3.115571 & -1.172147 & 0.786479 \\
\hline $\mathrm{H}$ & 2.256305 & -2.439831 & 1.155721 \\
\hline $\mathrm{O}$ & -0.086542 & -2.521101 & -0.741466 \\
\hline $\mathrm{H}$ & 0.315214 & -3.081389 & -1.420829 \\
\hline $\mathrm{H}$ & -1.051679 & -2.531826 & -0.875016 \\
\hline $\mathrm{O}$ & 2.186360 & 1.652189 & 0.857628 \\
\hline $\mathrm{H}$ & 2.278024 & 2.128642 & 1.694050 \\
\hline
\end{tabular}




$\begin{array}{crrr}\mathrm{H} & 3.082778 & 1.353123 & 0.577324 \\ \mathrm{O} & -0.227991 & 2.143106 & -1.411379 \\ \mathrm{H} & 0.323206 & 2.712274 & -1.967153 \\ \mathrm{H} & -0.927170 & 2.686061 & -1.013543 \\ \mathrm{O} & 4.049278 & 0.019823 & -0.248964 \\ \mathrm{H} & 4.959590 & 0.018007 & -0.575950 \\ \mathrm{H} & 3.413305 & -0.036150 & -1.029566 \\ \mathrm{O} & -0.062796 & -0.080617 & 2.509861 \\ \mathrm{H} & 0.367952 & -0.459313 & 3.289340 \\ \mathrm{H} & -0.856238 & 0.400696 & 2.794521\end{array}$

$\left[\left(\mathrm{NH}_{2} \mathrm{CH}_{2} \mathrm{COO}^{-}\right){ }^{1} \mathrm{La}\left(\mathrm{H}_{2} \mathrm{O}\right)_{6}(\mathrm{OH})\right]^{+}+\mathrm{H}^{+}(\mathrm{d})$ $\mathrm{E}=-1254.309264$

$\begin{array}{lrrr}\text { LA } & 0.391334 & 0.205794 & -0.000373 \\ \mathrm{C} & -2.411633 & -0.397461 & -0.332296 \\ \mathrm{O} & -1.563047 & -0.559990 & -1.285884 \\ \mathrm{C} & -3.874822 & -0.761736 & -0.576182 \\ \mathrm{O} & -2.019473 & 0.058686 & 0.798274 \\ \mathrm{~N} & -4.816724 & -0.559758 & 0.508758 \\ \mathrm{H} & -4.196362 & -0.199584 & -1.460636 \\ \mathrm{H} & -3.886274 & -1.812260 & -0.889739 \\ \mathrm{H} & -4.860253 & 0.416849 & 0.784794 \\ \mathrm{H} & -4.554919 & -1.096853 & 1.330363 \\ \mathrm{O} & -0.386117 & 2.162903 & -1.514903 \\ \mathrm{H} & -1.044233 & 2.122570 & -2.224601 \\ \mathrm{H} & 0.178682 & 2.937566 & -1.660777 \\ \mathrm{O} & 0.477846 & -2.383563 & -0.041126 \\ \mathrm{H} & -0.089053 & -2.882980 & -0.647371 \\ \mathrm{H} & 1.395467 & -2.724465 & -0.127192 \\ \mathrm{O} & 2.130069 & 2.257849 & -0.198013 \\ \mathrm{H} & 2.638048 & 1.622974 & -0.759753 \\ \mathrm{H} & 2.753969 & 2.721543 & 0.378182 \\ \mathrm{O} & 2.157188 & -0.585794 & 1.750395 \\ \mathrm{H} & 2.083774 & -0.783767 & 2.694522 \\ \mathrm{H} & 2.767400 & -1.247792 & 1.342503 \\ \mathrm{O} & 3.210364 & -2.186096 & -0.129333 \\ \mathrm{H} & 3.059656 & -1.426922 & -0.770310 \\ \mathrm{H} & 4.042702 & -2.628412 & -0.347423 \\ \mathrm{O} & -0.267324 & 1.440835 & 2.222485 \\ \mathrm{H} & -1.224839 & 1.262223 & 2.272915 \\ \mathrm{H} & -0.043660 & 2.112535 & 2.881081 \\ \mathrm{O} & 2.233400 & -0.092101 & -1.415508 \\ \mathrm{H} & 2.326393 & -0.151862 & -2.376823\end{array}$

$\left[\left(\mathrm{NH}_{2} \mathrm{CH}_{2} \mathrm{COO}^{-}\right){ }^{1} \mathrm{La}\left(\mathrm{H}_{2} \mathrm{O}\right)_{6}(\mathrm{OH})\right]^{+}+\mathrm{H}^{+}(\mathrm{e})$ $\mathrm{E}=-1254.300681$ 


$\begin{array}{lrrr}\text { LA } & 0.602762 & -0.014608 & 0.160157 \\ \mathrm{C} & -2.287107 & 0.226569 & -0.328121 \\ \mathrm{O} & -1.460429 & 0.705744 & -1.182359 \\ \mathrm{C} & -3.786487 & 0.382443 & -0.576466 \\ \mathrm{O} & -1.845969 & -0.360186 & 0.722632 \\ \mathrm{~N} & -4.703538 & -0.190394 & 0.391827 \\ \mathrm{H} & -3.972476 & 1.458673 & -0.674029 \\ \mathrm{H} & -3.987306 & -0.030895 & -1.571431 \\ \mathrm{H} & -4.540830 & 0.185744 & 1.321460 \\ \mathrm{H} & -4.598557 & -1.199035 & 0.450340 \\ \mathrm{O} & 0.257548 & 2.654673 & 0.819692 \\ \mathrm{H} & -0.511992 & 3.221384 & 0.970969 \\ \mathrm{H} & 0.583285 & 2.308305 & 1.685914 \\ \mathrm{O} & 0.624206 & -2.274200 & -1.313169 \\ \mathrm{H} & 0.510134 & -2.070235 & -2.256930 \\ \mathrm{H} & 0.141312 & -3.089427 & -1.111146 \\ \mathrm{O} & 2.942405 & -1.263277 & 0.035096 \\ \mathrm{H} & 3.681390 & -1.250621 & 0.660436 \\ \mathrm{H} & 2.987720 & -2.096169 & -0.459535 \\ \mathrm{O} & 2.281509 & 1.878289 & -0.809361 \\ \mathrm{H} & 3.243081 & 1.951818 & -0.727342 \\ \mathrm{H} & 1.895631 & 2.666539 & -0.381322 \\ \mathrm{O} & 0.684615 & 0.109902 & -2.643020 \\ \mathrm{H} & 1.276789 & 0.716947 & -3.111501 \\ \mathrm{H} & -0.211521 & 0.499130 & -2.679970 \\ \mathrm{O} & -0.102833 & -2.219222 & 1.526775 \\ \mathrm{H} & -1.033502 & -1.951723 & 1.652801 \\ \mathrm{H} & 0.235534 & -2.481609 & 2.395169 \\ \mathrm{O} & 1.322452 & 0.653711 & 2.138859 \\ \mathrm{H} & 1.682167 & 0.589035 & 3.030771\end{array}$

$\left[\left(\mathrm{NH}_{2} \mathrm{CH}_{2} \mathrm{COO}^{-}\right){ }^{1} \mathrm{La}\left(\mathrm{H}_{2} \mathrm{O}\right)_{6}(\mathrm{OH})\right]^{+}+\mathrm{H}^{+}(\mathrm{g})$ $\mathrm{E}=-1254.299054$
$\begin{array}{llll}\text { LA } & 0.511951 & 0.004365 & 0.012455\end{array}$
$\begin{array}{llll}\text { C } & -2.363077 & -0.063098 & -0.206643\end{array}$
$\begin{array}{llll}\mathrm{O} & -1.648800 & -0.614408 & -1.129512\end{array}$
$\begin{array}{llll}\text { C } & -3.884759 & -0.173275 & -0.282976\end{array}$
$\begin{array}{llll}\mathrm{O} & -1.791470 & 0.557659 & 0.749474\end{array}$
$\begin{array}{llll}\mathrm{N} & -4.663837 & 0.483361 & 0.751008\end{array}$
$\mathrm{H} \quad-4.181700 \quad 0.201212 \quad-1.269606$

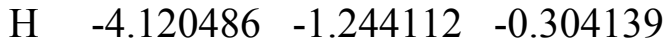
$\begin{array}{llll}\mathrm{H} & -4.492826 & 1.485083 & 0.757593\end{array}$
$\begin{array}{llll}\mathrm{H} & -4.413499 & 0.136192 & 1.672675\end{array}$
$\begin{array}{llll}\mathrm{O} & -0.056336 & 1.917145 & -1.708905\end{array}$
$\begin{array}{llll}\mathrm{H} & -0.867935 & 1.942790 & -2.237414\end{array}$
$\mathrm{H} \quad 0.053492 \quad 2.787361 \quad-1.291867$ 


$\begin{array}{lrrr}\mathrm{O} & -0.023699 & -2.288200 & 1.197347 \\ \mathrm{H} & -0.196469 & -3.081289 & 0.667573 \\ \mathrm{H} & -0.356839 & -2.450384 & 2.091671 \\ \mathrm{O} & 1.493786 & 2.525900 & 0.546665 \\ \mathrm{H} & 2.271946 & 3.093748 & 0.461194 \\ \mathrm{H} & 1.507619 & 2.127781 & 1.455643 \\ \mathrm{O} & 3.009304 & -0.886261 & 0.932859 \\ \mathrm{H} & 2.632595 & -0.445736 & 1.740150 \\ \mathrm{H} & 3.355285 & -1.747664 & 1.205900 \\ \mathrm{O} & 2.647922 & 0.185590 & -1.558515 \\ \mathrm{H} & 3.406625 & -0.032346 & -0.988637 \\ \mathrm{H} & 2.943636 & 0.802876 & -2.242376 \\ \mathrm{O} & 0.454082 & -2.138948 & -1.626892 \\ \mathrm{H} & 1.017811 & -2.432030 & -2.357210 \\ \mathrm{H} & -0.453029 & -2.021919 & -1.968206 \\ \mathrm{O} & 1.172557 & 0.506380 & 2.163604 \\ \mathrm{H} & 0.787696 & 0.545500 & 3.048798\end{array}$

